2014

\title{
FABRICATION OF DRUG ELUTING TI IMPLANTS FOR DENTAL/ ORTHOPEDIC APPLICATIONS
}

Azhang Hamlekhan

Michigan Technological University

Follow this and additional works at: https://digitalcommons.mtu.edu/etds

Part of the Biomedical Engineering and Bioengineering Commons, and the Nanoscience and Nanotechnology Commons

Copyright 2014 Azhang Hamlekhan

\section{Recommended Citation}

Hamlekhan, Azhang, "FABRICATION OF DRUG ELUTING TI IMPLANTS FOR DENTAL/ORTHOPEDIC APPLICATIONS", Dissertation, Michigan Technological University, 2014.

https://doi.org/10.37099/mtu.dc.etds/961

Follow this and additional works at: https://digitalcommons.mtu.edu/etds

Part of the Biomedical Engineering and Bioengineering Commons, and the Nanoscience and Nanotechnology Commons 


\title{
FABRICATION OF DRUG ELUTING TI IMPLANTS FOR DENTAL/ORTHOPEDIC APPLICATIONS
}

$$
\text { By }
$$

\author{
Azhang Hamlekhan
}

\begin{abstract}
A DISSERTATION
Submitted in partial fulfillment of the requirements for the degree of DOCTOR OF PHILOSOPHY
\end{abstract}

In Mechanical Engineering-Engineering Mechanics

MICHIGAN TECHNOLOGICAL UNIVERSITY

2014

(C) 2014 Azhang Hamlekhan 
This dissertation has been approved in partial fulfillment of the requirements for the Degree of DOCTOR OF PHILOSOPHY in Mechanical Engineering-Engineering Mechanics.

Department of Mechanical Engineering-Engineering Mechanics.

Dissertation Co-Advisor: Prof. Tolou Shokuhfar

Dissertation Co-Advisor: Prof. Cortino Sukotjo

Committee Member: Prof. Craig Friedrich

Committee Member: Prof. Christos Takoudis

Committee Member: Prof. Reza Shahbazian Yassar

Committee Member: Prof. Mathew T. Mathew

Department Chair: Prof. William W. Predebon 
To my parents 


\section{Table of Contents}

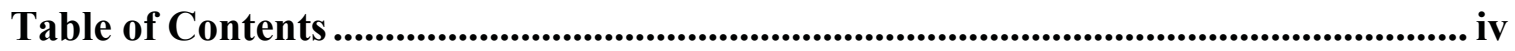

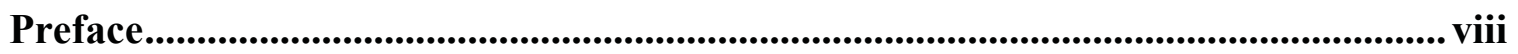

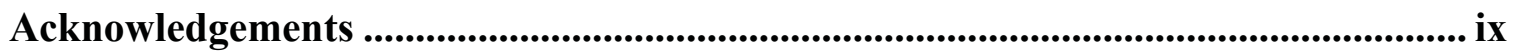

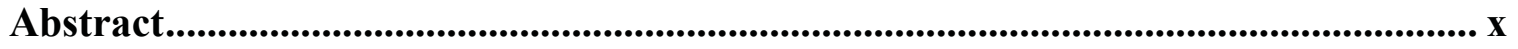

Chapter 1 ............................................................................................................................... 1

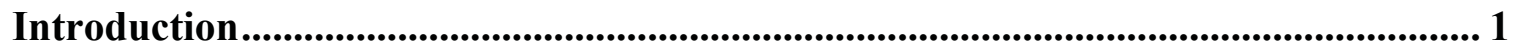

Chapter 2 .......................................................................................................................... 5

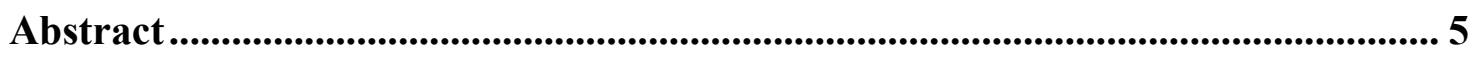

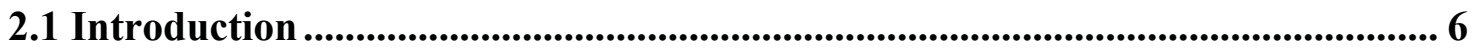

2.2 Nanotube development ............................................................................ 7

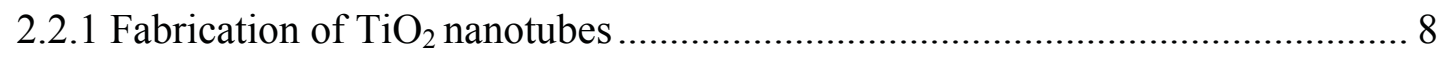

2.2.2 Mechanism of nanotube formation during anodization.................................. 9

2.3 In vivo performance of surface with nanotube................................................. 11

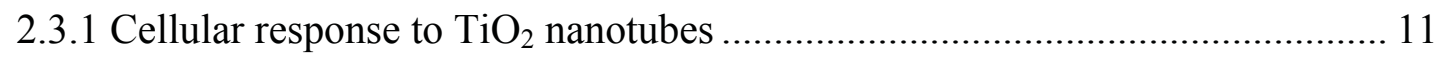

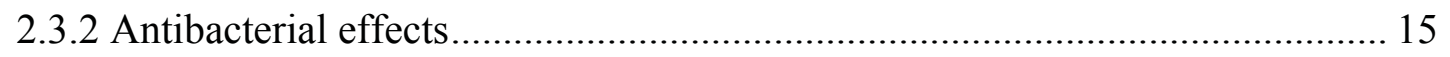

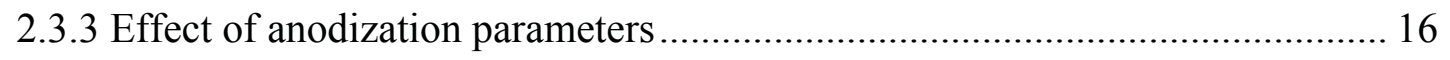

2.4 Controlling TNTs dimensions ............................................................. 21

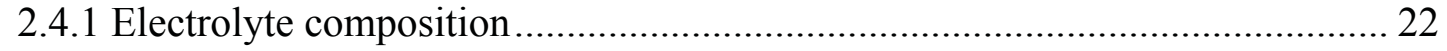

2.5 Nanotube development on zirconium surface ..................................................... 23

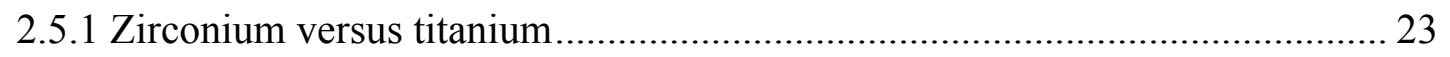

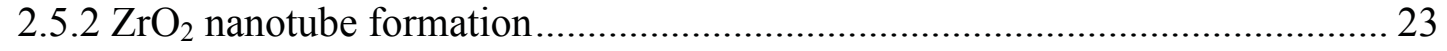

2.5. 3 Cellular behavior on zirconium nanotube ................................................ 25

2.6 Bone Implant Contact (BIC) and Bone Mineral Deposition (BMD) ............... 25

2.7 Drug loading on nanotubes .......................................................................................... 26

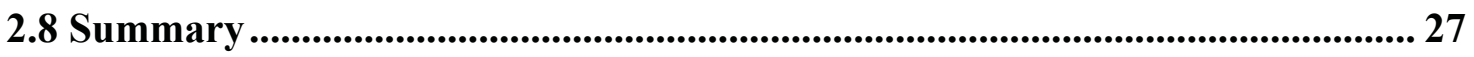

Acknowledgments ..................................................................................................... 28

Chapter 3 ............................................................................................................................ 29

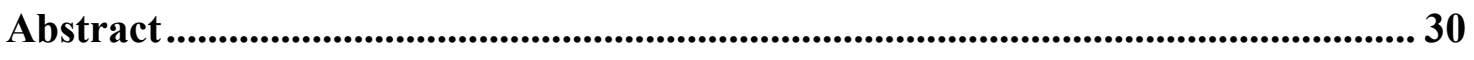

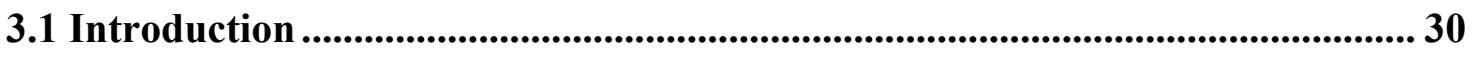




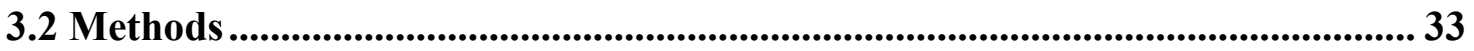

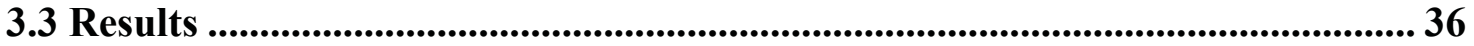

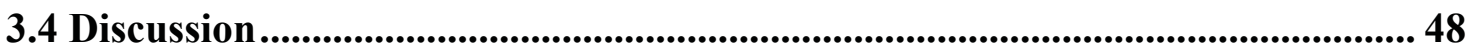

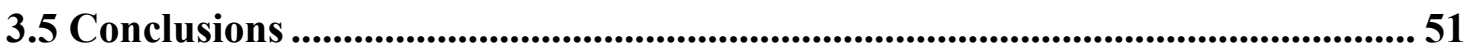

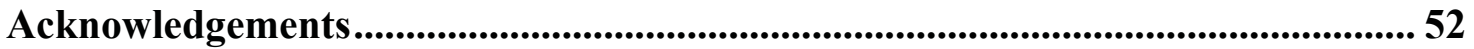

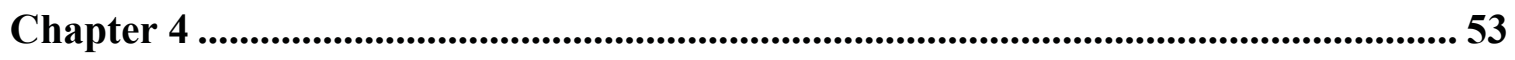

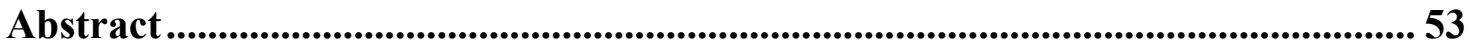

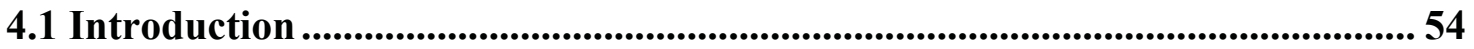

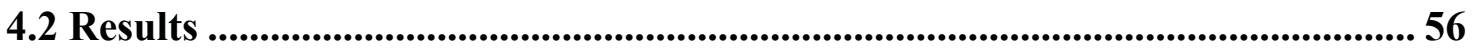

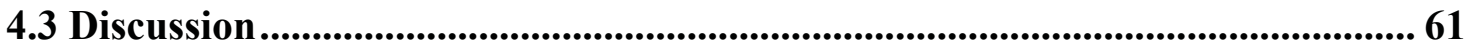

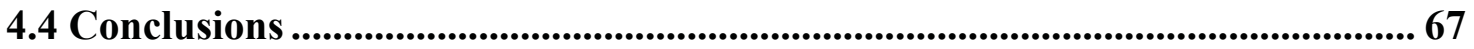

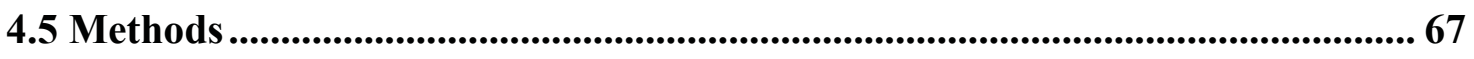

Acknowledgements................................................................................................6 69

Chapter 5 [................................................................................................................................... 71

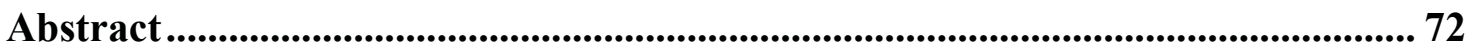

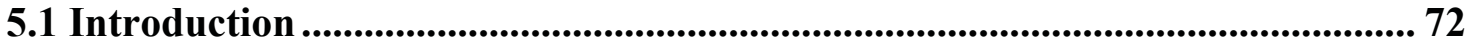

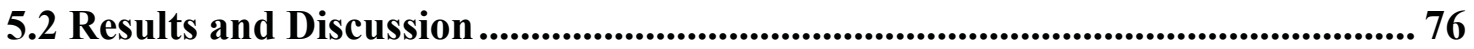

5.3 Experimental......................................................................................................... 92

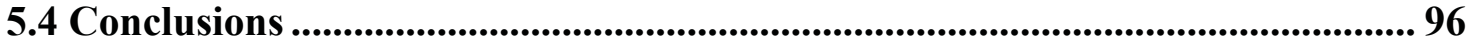

Acknowledgements ...................................................................................................... 97

Chapter 6 ....................................................................................................................... 98

Abstract ....................................................................................................................... 99

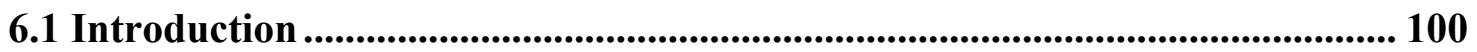

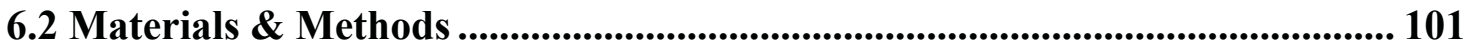

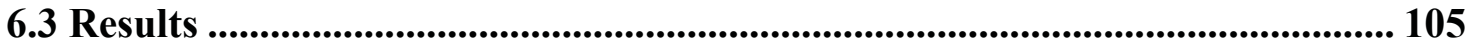

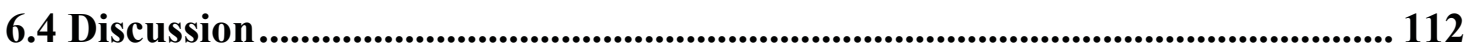

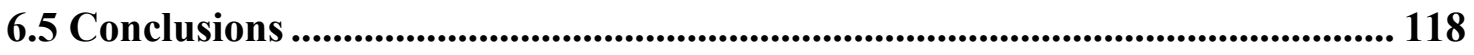

Acknowledgements ........................................................................................................ 118

Chapter 7 ................................................................................................................................. 119

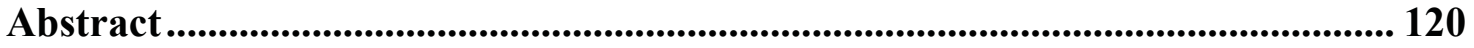




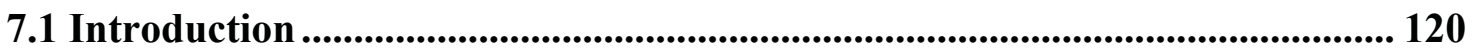

7.2 Materials and Methods ............................................................................................. 123

7.3 Results .............................................................................................................................. 128

7.4 Discussion ............................................................................................................. 142

7.5 Conclusion ....................................................................................................................... 150

Acknowledgements............................................................................................................... 151

Chapter 8 ......................................................................................................... 152

Discussion and Conclusions ........................................................................................... 152

Chapter 9 ................................................................................................................ 156

Future Work....................................................................................................................... 156

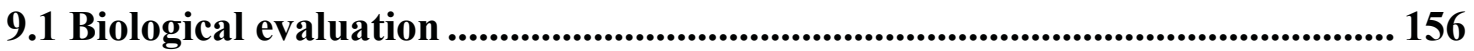

9.2 Determining controlled drug release profiles ........................................................ 157

9.3 Fabrication of smart drug eluting implants............................................................. 157

9.4 Optimization of surface properties.................................................................... 158

Appendix .............................................................................................................................. 159

Appendix 1: Copyright for reproduction of figure 2.2 .............................................. 160

Appendix 2: Copyright for reproduction of figure 2.3 ................................................. 161

Appendix 3: Copyright for reproduction of figure 2.4 ............................................... 162

Appendix 4: Copyright for reproduction of figure 2.5 .............................................. 163

Appendix 5: Copyright for reproduction of figure 2.6 ................................................ 164

Appendix 6: Copyright for reproduction of figure 2.7 ................................................ 165

Appendix 7: Copyright for reproduction of figure 2.8 ............................................. 166

Appendix 8: Copyright for reproduction of figure 2.9 ................................................ 167

Appendix 9: Copyright for the Manuscript Submitted to the journal of PLOS

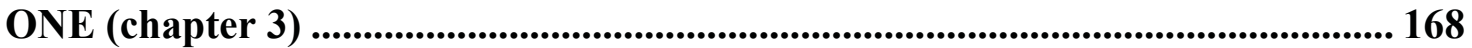

Appendix 10: Copyright for the Manuscript Submitted to the journal of Advanced Healthcare Materials (chapter 4)................................................................. 169

Appendix 11: Copyright for the Manuscript Submitted to the journal of Journal of Materials Chemistry B (chapter 5)......................................................................... 170

Appendix 12: Copyright for the Manuscript Submitted to the Journal of Oral Implantology (chapter 6) .................................................................................................... 171 
Appendix 13: Copyright for the Manuscript Submitted to the Journal of Electrochimica Acta (chapter 7) ................................................................. 172

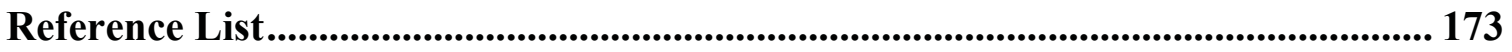




\section{Preface}

The material included in this dissertation is prepared independently by Azhang Hamlekhan and collaboratively with other researchers. All materials presented in chapters 2-7 are obtained under guidance of and using the facilities and equipments provided by Prof. Tolou Shokuhfar, Prof. Cortino Sukotjo, Prof. Christos Takoudis and Prof. Mathew T. Mathew. Azhang Hamlekhan, Arman Butt, Sweetu Patel, Dmitry Royhman and John Grotberg have performed the experiments, collected and analyzed the data. Manuscripts are written by the first author and co-authors have provided critical comments to improve the final version.

Prof. Alexander L. Yarin and Prof. Suman Sinha-Ray have provided guidance and analyzed the data presented in chapter 4. Prof. Gregory Jursich has performed part of experiments presented in chapters 3 and 5 and analyzed the obtained data. Prof. Judy Yuan has performed the statistical analysis of the data presented in chapters 3 and 5. Prof.

Reza Shahbazian-Yassar has provided guidance for preparation of chapter 2. Prof. William Hendrickson has provided the lab space to perform part of experiments. 


\section{Acknowledgements}

I would like to express my special gratitude to my advisor professor Tolou Shokuhfar, who has been an inspirational guide for me. Her invaluable guidance paved the way for me to grow as a research scientist and develop my career. My thanks and appreciations also go to my co-advisor professor Cortino Sukotjo for his motivational advices and providing me with clinical perspective towards modification of implants.

I would like to acknowledge my committee members, professor Tolou Shokuhfar, professor Cortino Sukotjo, professor Craig Friedrich, professor Christos Takoudis, professor Reza Shahbazian Yassar and professor Mathew T. Mathew who made my work possible. I also appreciate all contributions of my colleagues Arman Butt, Sweetu Patel, Dmitry Royhman and John Grotberg in performing the experiments.

Lastly, yet importantly, I would like to thank my family for all their encouragements. A special thank to my parents whose emotional support has made my achievements possible. 


\begin{abstract}
Titanium and its alloys are typically used for fabrication of dental and orthopedic implants as they posses various desirable properties including biocompatibility and corrosion resistance. In spite of such benefits, titanium implants show lack of osseointegration after surgery in minor cases. The objective of this research has been to modify the surface of titanium alloy for medical applications through increasing surface hydrophilicity and drug loading. Primarily, anodization method is employed for fabrication of nanotubes on titanium surface to act as anchoring cite for cells.

Considering the key role of surface hydrophilicity on cellular attachment to the surface and subsequent biological behavior of attached cells, the fabrication condition of nanotubes during anodization and following heat treatment is optimized. It is shown that anodization voltage, anodization duration and heat treatment temperature and duration can be controlled to fabricate a nanotubular surface that maintains its hydrophilicity over a long period of time. In order to verify the role of surface morphology on obtained characteristics, smooth, anodized-smooth, rough and anodized-rough surfaces are explored. The results show that anodized-smooth and anodized-rough surfaces show higher hydrophilicity than non anodized surfaces. Hydrophilic nanotubes not only promote cell adsorption; but also increase absorption of aqueous drug solution. Consequently, nanotubes are successfully loaded with drug and act as nano drug reservoirs that are potential to deliver the loaded drug locally after surgery. It is shown that dimension of nanotubes can affect rate of drug release. In fact the results indicate that nanotubes with higher aspect ratio (ratio of length to diameter) prolong drug release. A novel method for fabrication of naotubes was investigated which suggests a new way for controlling length of nanotubes. It is shown that heat treatment of the substrate prior to anodization affects length of nanotubes obtained eventually after anodization. In fact, the results show that anatase crystalline structure affects mechanism of formation of nanotubes to form longer nanotubes. Finally, it is demonstrated that corrosion resistance of heat treated nanotubular surface is higher than either heat treated surface or nanotubular surface alone.
\end{abstract}




\section{Chapter 1}

\section{Introduction}

Various biomaterials are employed for treatment of bone defects and substituted for bone as implants. Among these materials, titanium and titanium alloys have recently attracted attention due to their desirable properties. Titanium is biocompatible, corrosion resistant and possesses proper mechanical properties to be used as bone/dental implants. Despite all these benefits, titanium implants sometimes become loose after surgery which results in infection and implant failure. Several surface modifications have been developed to overcome this issue and increase osseointegration to bone tissue. In current dissertation, the anodization method is explored for fabrication of nanotubes on the surface of titanium. In addition capability of nanotubes to be loaded drug and their potential for providing prolonged local drug delivery is investigated. Previous studies show that presence of these nanostructures promotes desirable cellular behavior in comparison with smooth surface of conventional implants. Increase of cell attachment, proliferation and differentiation on nanotubular surface is attributed to the increase of surface area, surface energy and increase of surface wettability after anodization.

In chapter 2, recent progress toward surface modification of bone/dental implants with metal oxides is reviewed with an emphasis on application of titanium dioxide nanotubes. This chapter presents an overview of various methods used for development of nanotubular structures with an emphasis on anodization technique, mechanism of nanotube formation during anodization and biological evaluation including response of different cell lines on anodized surface. In addition impact of annealing of anodized surface, increase of surface roughness and increase of wettability on promotion of desirable cellular behavior is discussed. Considering the role of nanotube dimensions on cellular fate, influence of anodization condition parameters on diameter and length of resultant nanotubes is summarized. Finally capability of nanotubes in acting as drugreservoirs is taken into account. Although nanotubes are able to be loaded with drug and 
provide local drug delivery, controlling drug release from these drug-reservoirs remains to be a challenge.

In chapter 3, effect of anodization condition and annealing of anodized surface on hydrophilicity of obtained nanotubes over time is reported. In fact, fabrication conditions are optimized to produce anti-aging $\mathrm{TiO} 2$ nanotubes that are able to maintain their hydrophilicty over time. This study was designed based on the fact that improvement of cellular response on anodized surface is attributed to enhancement of surface roughness, available surface area and hydrophilicity. Nanotubular surface is hydrophilic and surface hydrophilicity is further increased after anodized surface is annealed. In this study, hierarchical micro-nano scale topography of $\mathrm{TiO} 2$ is formed on the substrate via acidetching and anodization respectively, in order to mimic natural bone morphology. Although it is expected that a roughened surface on titanium and its alloys provides long term mechanical interlocking ability, it has not been explored to the extent of polished surfaces. Considering that surface hydrophilicity plays a key role on cellular fate, effect of various anodization and annealing condition on surface hydrophilicity is verified. Monitoring hydrophilicity of the obtained $\mathrm{TiO} 2$ nanotubes over time demonstrates that maintenance of hydrophilicity is affected by anodization voltage and annealing temperature. Results show that anodization and annealing condition can be adjusted to enhance implant surface hydrophilicity, promote cellular behavior and consequently increase osseointegration. In addition, fabrication conditions should be taken into account to determine shelf-life of implants.

In chapter 4, the role of nanotubes aspect ratio for controlling rate of drug release is reported. In fact, aspect ratio of nanotubes is optimized for prolonged release of drug molecules. This study was performed to overcome the existing challenges in prolongation of drug release from nanotubes and to introduce a simple novel method. To achieve this objective, 9 groups of nanotubes with different diameters and lengths are fabricated. All groups are loaded with a model drug and then rate of drug release over time is monitored. The relation of the nanotube dimensions (volume, length, diameter and aspect ratio) to the drug release rate is determined. The findings indicate that an increase in any of these factors increases prolongs release of drug. However, the strongest parameter influencing 
the release rate is the aspect ratio. It is concluded that nanotubes with higher aspect ratios demonstrate prolonged release. A theoretical model is suggested for kinetics of drug release which is shown to be in agreement with the experimental data. These scientific discoveries can eventually be used for fabrication of drug eluting implants.

In order to promote cellular viability on the surface of titanium and its alloys, various studies are performed to optimize wettability, roughness and composition of the surface. In chapter 5, it is reported how surface characteristics of titanium alloy is enhanced for biomedical applications through integrated anodization and annealing treatments. Results demonstrate that desirable surface characteristics are provided by anodized samples after annealing. Water contact angle measurements of smooth, rough, anodized-smooth and anodized-rough surfaces before thermal oxidation reveals that anodized-smooth and anodized-rough substartes are super-hydrophilic while nonanodized smooth and rough surfaces show water contact angle of higher than $50^{\circ}$. Following thermal oxidation at $450{ }^{\circ} \mathrm{C}$ for 3 hours, surface of all samples become superhydrophilic. When samples are exposed to air for a period of 3 weeks, smooth and rough surfaces lose their hydrophilicity, while anodized-smooth and anodized-rough surfaces remain hydrophilic.

In chapter 6 , formation of nanotubes on thermally oxidized surface of titanium alloy is investigated. Conventionally, thermal oxidation is performed after formation of nanotubes and transformation into crystalline structure is reported to increase cellular viability. Heat treatment of titanium is performed at temperatures higher than 200 and $400{ }^{\circ} \mathrm{C}$ to form anatase and rutile crystalline structures respectively. Here, effect of thermal oxidation at various temperatures on formation of nanotubes is explored to determine the role of crystalline structure of bulk substrate from which nanotubes grow. Nanotubes are fabricated on non-heat treated, 300 and $600{ }^{\circ} \mathrm{C}$ heat treated surfaces. The results show that heat treatment prior to anodization can significantly influence obtained nanotubes in terms of length and surface morphology while chemical composition remains the same. 
In chapter 7, the corrossion of titanium is investigated in response to anodization and thermal oxidation in presence of serum proteins, at physiological temperature. Various parameters were evaluated to determine corrosion behavior including corrosion potential, polarization resistance, open circuit potential, passivation current density and corrosion current density. The results show that the highest corrosion resistance is provided by Anodized+Thermally Oxidized samples followed by Anodized samples, then Smooth samples and finally Thermally Oxidized samples.

In chapter 8 major results of the research are overviewed briefly and discussed to reach the prominent conclusions of this study. Based on the conclusions, the future work is determined and explained in chapter 9 . 


\title{
Chapter 2
}

\section{Recent Progress toward Surface Modification of Bone/Dental Implants with Titanium and Zirconia Dioxide Nanotubes*}

\author{
Azhang Hamlekhan ${ }^{1}$, Christos Takoudis ${ }^{2,3}$, Cortino Sukotjo ${ }^{4}$, Mathew T. Mathew ${ }^{5}$, \\ Amarjit Virdi $^{6}$, Reza Shahbazian-Yassar ${ }^{1,7,8}$, Tolou Shokuhfar ${ }^{1,7,8,9}$ \\ ${ }^{1}$ Mechanical Engineering-Engineering Mechanics, Michigan Technological University, \\ Houghton, MI, 49931 \\ ${ }^{2}$ Department of Chemical Engineering, University of Illinois at Chicago, Chicago, IL, \\ 60607 \\ ${ }^{3}$ Department of Bioengineering, University of Illinois at Chicago, Chicago, IL, 60607 \\ ${ }^{4}$ Department of Restorative Dentistry, College of Dentistry, University of Illinois at \\ Chicago, Chicago, IL, 60612 \\ ${ }^{5}$ Department of Orthopedics, Rush University Medical Center, Chicago, IL, 60612 \\ ${ }^{6}$ Department of Anatomy and Cell Biology, Orthopedic Surgery, Rush University, \\ Chicago, IL, 60612 \\ ${ }^{7}$ Department of Physics, University of Illinois at Chicago, Chicago, IL, 60607 \\ ${ }^{8}$ Department of Mechanical and Industrial Engineering, University of Illinois at \\ Chicago, Chicago, IL, 60607 \\ ${ }^{9}$ Corresponding author, tshokuhf@mtu.edu
}

\begin{abstract}
Fabrication of titanium dioxide nanotubes (TNTs) and zirconia dioxide nanotubes (ZrNTs), through electrochemical anodization method on metal substrate, has shown great potential in biomedical purposes. As a modified surface, nanotubular surfaces

*The material contained in this chapter has been submitted to the open access Journal of Nanotechnology and Smart Materials. As the first author of this publication, I have done the literature review and writing.
\end{abstract}


promote cellular interaction compared with conventional flat or polished surfaces. In this study we review different aspects of improvements achieved by growing metal oxide nanotubes. ZrNTs and TNTs have been shown to be promising candidates for application as orthopedic or dental implants. This paper presents an overview of anodization techniques used to produce nanotubular structures (specifically TNTs), subsequent properties of these anodized surfaces, and eventually in vitro as well as in vivo biological responses pertinent to clinical applications.

Keywords: $\mathrm{TiO}_{2}$, nanotube, $\mathrm{ZrO}_{2}$ nanotube, anodization, drug loading, drug release, dental application, orthopedics, osseointegration, cellular response, cytotoxicity, surface modification, surface treatment, bone implants, dental implants, oral and maxillofacial implants

\subsection{Introduction}

Different categories of biomaterials have been employed to repair bone injuries including metals, polymers, ceramics, as well as their composites and natural materials. Although polymers have shown appropriate primary fixation, they are potential to release monomers in the body which results in inflammation and degradation of implant [1]. Ceramics and bioglasses provide higher biocompatibility and compression strength compared to biopolymers; however, they suffer from low fracture toughness and higher elastic modulus compared to bone. Ceramics, in the form of nanoparticles, are employed in polymeric matrix in order to fabricate composites that benefit from advantages of both ceramics and polymers at the same time [2-4]. Biometals, such as stainless steel and cobalt chromium alloy, have high mechanical properties but they release nickel and are potential to cause allergenic response and adverse reaction due to corrosion [5].

Compared with other biometals used as implants, titanium and its alloys have recently attracted attention [6] as they provide great biocompatibility in terms of low ion release [7], excellent corrosion resistance [8], great mechanical properties in terms of high hardness, low elastic modulus and low density [9-14]. Surface characteristics initiate from presence of a native oxide layer on the surface. When titanium is exposed to air, a 
layer of titania $\left(\mathrm{TiO}_{2}\right)$ with thickness of $2-5 \mathrm{~nm}$ is formed on its surface that protects the bulk material from corrosion [15] and makes it bioinert [16]. However, in minor cases they are encapsulated by fibrous tissue in vivo and lack osteointegrity [17]. In addition low $\mathrm{pH}$ and presence of lipopolysaccharide in saliva enhances corrosion rate of titanium dental implant [18]. In order to develop bioactivity and osteointegration, different surface modifications have been performed.

Anodization technique of titanium leading to the formation of Titanium Oxide Nanotubes (TNTs) on the surface has attracted much attention lately [19-25]. The anodized TNT surface possesses promising potentials for biomedical application [26], since it has shown to be able to increase osteoblast cell adhesion and desirable functions [19-21], increase growth of hydroxyapatite, [27,28] and influence cellular behavior to enhance tissue integration [29].

In this study, different mechanisms of formation of TNT are reviewed and anodization technique is elaborated as the most investigated method. Cellular response to TNTs, with an emphasis on bone cells behavior, is taken into consideration. It is discussed how cellular response can be controlled by different parameters including crystallinity, roughness, wettability and TNTs dimension. Anodization of titanium alloys is briefly explained and finally success of nanotubular implants in in vivo experiments and their potential for drug release purpose is overviewed.

\subsection{Nanotube development}

In order to develop bioactivity and osteointegration, different surface modifications of titanium have been done including hydroxyapatite and calcium phosphate coating [30]. However these coatings result in delamination at hydroxyapatite and titanium interface because of difference in mechanical moduli [31]. Later studies demonstrated that early healing of pre-implant soft tissue is affected by topography of titanium surface [32]. Recently surface of implants have been modified by taking advantage of nanotechnology. Titanium nanostructured surfaces provide more surface area for protein adsorption and as a result more cellular interaction [33]. Being integrated with bulk substrate, they also prevent delamination deficiency [34] and improve osteointegration of the implant. 


\subsubsection{Fabrication of $\mathrm{TiO}_{2}$ nanotubes}

\section{Assisted-template method}

Assisted-template method is performed either by positive or negative templates. A positive template is used for coating oxide layer on the outer surface of template while a negative template is used to coat its inside porosities [35]. For both types of templates, anodic aluminum oxide (AAO) membrane is commonly used as template which holds scattered cylindrical pores with uniform dimensions in its structure (figure 2.1) [36-39].

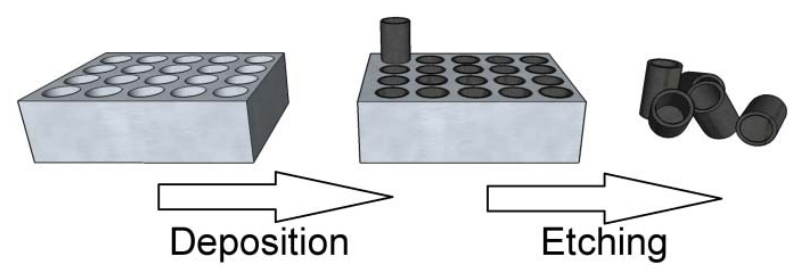

Figure 2.1 Fabrication of nanotubes via a porous hard template such as AAO.

\section{Hydrothermal treatment}

Hydrothermal treatment method is started by $\mathrm{NaOH}$ treatment of $\mathrm{TiO}_{2}$ nanoparticles. Electrostatic repulsion of the charge on sodium results in extension of $\mathrm{TiO}_{2}$ nanoparticles to form nano-sheets. After washing with $\mathrm{HCl}$, electrostatic charges are removed and sheets scroll to become $\mathrm{TiO}_{2}$ nanotubes [37]. A major advantage of this method is obtaining pure phase $\mathrm{TiO}_{2}$ nanotubes with good crystallinity [36]. Disadvantages include long reaction times and the application of $\mathrm{NaOH}$, which can cause production of nanotubes that are in powder form of random alignment [36,37].

\section{Anodization}

In this review we have focused on anodization as the most investigated method of fabricating nanotubes since it has shown to be well promising in order to enhance desirable surface characteristics and cellular response. Through this method, surface of the material is modified by formation of nanotubes while the bulk material is employed 
as anode of an electrochemical cell (figure 2.2). Advantages of this method include production of nanotubes with ordered alignment, high aspect ratio and possibility of controlling TNTs dimensions by varying the anodization conditions [36,37]. Anodization of pure titanium leads to formation of a uniform nanotubular layer on the substrate. When titanium alloys, Ti6Al7Nb and Ti6Al4V are anodized, the nanotubular structure is produced on alpha phase (Al-rich phase) with diameter of $100 \mathrm{~nm}$ and spacing of $50 \mathrm{~nm}$. However, the beta phase ( $\mathrm{Nb}$-rich phase of Ti6Al7Nb and V-rich phase of Ti6Al4V) behaves differently for these alloys during anodization. Beta phase of Ti6Al7Nb produces nanotubes with diameter of $50 \mathrm{~nm}$ while beta phase of Ti6A14V is dissolved [40].

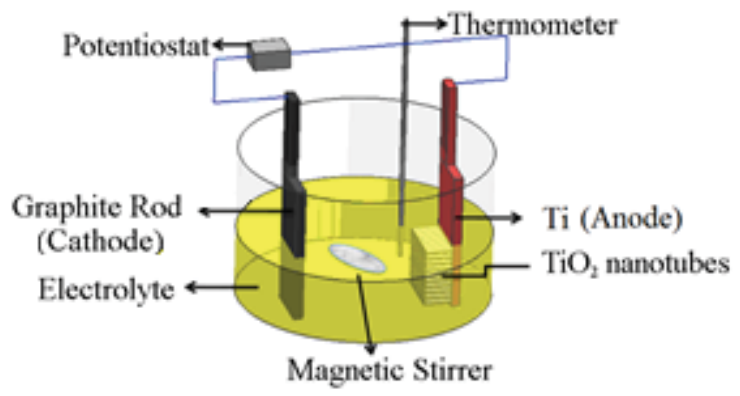

Figure 2.2 Electrochemical etching anodization set-up for the synthesis of TNT.

Reproduced by permission of The Royal Society of Chemistry [41].

\subsubsection{Mechanism of nanotube formation during anodization}

When valve metals are used as an anode of electrochemical cell different oxide layer structures may be formed depending on anodization condition. Theses morphologies include electropolished surface, compact anodic oxides, rapid (disorganized) oxide nanotube and ordered nanoporous or nanotubular layers. Therefore only by establishing particular conditions, anodization can be applied to produce ordered porous layer or aligned nanotube structure on some of transition metals including Ti. When conditions are proper for nanotube formation, anodization begins by dissolution of valve metal in electrolyte as cation [42]. The dissolved cation reacts with $\mathrm{O}_{2}$ from electrolyte and forms an oxide layer which is deposited on metal surface. Oxide 
formation continues at metal-oxide interface. As the thickness of oxide layer increases the field decreases and consequently the process is stopped. Therefore the thickness of compact layer is dependent on applied voltage. During the next stage, pores are formed on the surface of compact oxide layer that gradually grow into tubular shape (figure 2.3). Since nanotube wall boundaries are etched the tubes become separated. As the anodization duration increases tubes are elongated. Finally a steady state condition is established under which a competition exists between oxide formation at the bottom of tubes and cation dissolution at electrolyte interface. Length remains constant under steady state condition $[6,43,44]$.

Several reasons have been hypothesized for formation of tubular structure from compact oxide layer. One hypothesis explains that the surface fluctuation that exists on compact oxide layer enhances the electrical field. As a result transportation of ions is accelerated in these regions and field enhanced dissolution causes formation of pores on compact oxide layer. Another hypothesis is based on volume expansion when metal transforms to oxide. Increase of volume causes stress at the interface of metal/oxide that leads to upward flow of oxide and formation of nanotubes. Local acidity in the tubes is also assumed as a factor that enhances tubular formation $[43,44]$.

Presence of fluoride ion in electrolyte greatly affects the produced surface structure. Low concentration of fluoride in electrolyte leads to formation of compact oxide layer. Fluoride ion causes chemical etching of the oxide layer and its presence at intermediate concentration is required for $\mathrm{TiO}_{2}$ tubular formation from compact oxide. However, it also dissolves $\mathrm{Ti}^{4+}$ and forms $\mathrm{TiF}_{6}$ complex in water. Therefore, when its concentration is high, the surface is electro-polished. Having small ionic radius, fluoride migrates faster than $\mathrm{O}_{2}$ in oxide layer and accumulates in oxide/metal interface. During plastic flow of oxide to form tubular structure, accumulated fluoride moves to tube wall boundaries. Later fluoride ion causes etching of boundaries and separation of tubes [45].

In fluoride containing electrolytes, the top part of $\mathrm{TiO}_{2}$ nanotubes is gradually etched and as a result a V-shaped profile is created. Also hexagonal porous structure of the base of nanotubes transforms into tubular structure at the top cross-section. Application of non-aqueous electrolytes such as glycerol or ethylene glycol leads to formation of highly ordered $\mathrm{TiO}_{2}$ nanotubes. The highest order is achieved under 
maximum current. Several other valve metals, such as $\mathrm{Ta}, \mathrm{Hf}, \mathrm{Mg}, \mathrm{Fe}, \mathrm{W}, \mathrm{Nb}$, and $\mathrm{Zr}$ can be formed into organized nanotubular or nanoporous structures by applying principles used for formation of $\mathrm{TiO}_{2}$ nanotubes. The nanostructure produced on the metals is amorphous which can be annealed to form crystalline phase $[43,44]$.

(a)

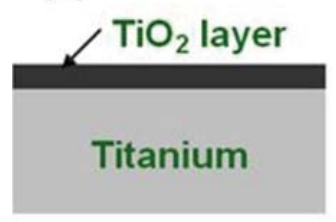

(d)

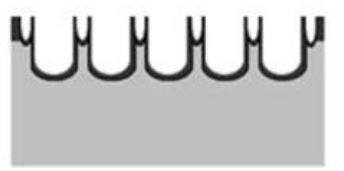

(b)

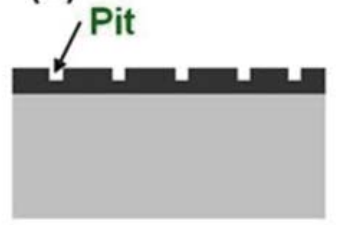

(c)

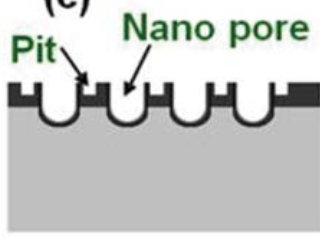

(e) $\mathrm{TiO}_{2}$ Nanotube

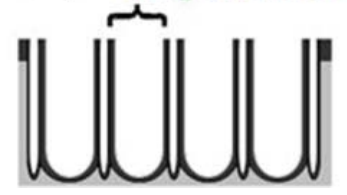

Figure 2.3 Schematic illustration of $\mathrm{TiO}_{2}$ nanotube formation [46].

Reproduced from Jin et al.

\subsection{In vivo performance of surface with nanotube}

\subsubsection{Cellular response to $\mathrm{TiO}_{2}$ nanotubes}

As a biomaterial is exposed to in vitro condition or in vivo physiologic environment, proteins of cell culture media or body fluids adsorb to its surface in less than a second. The adsorbed protein functional groups (ligands), interact with surface receptors of the cells (integrins) [47]. Desirable cellular response of different cell lines is increased on TNT surfaces compared to flat machined surface. Such enhancement is due to increase of surface area that provides more area for cell-substrate interaction, more surface energy, more protein adsorbtion and as a result higher cell adhesion [9,34,48-51]. In addition, nano-topography of surface mimics natural environment for cells and provides integrin clustering. In the human body, bone cells interact with the fluid that flows around them in interstitial spaces [52]. Presence of space between tubes can be helpful for transport of waste and nutrients and therefore cell metabolism [34]. 
Wettability is also sharply increased after anodization of flat titanium which enhances cell adhesion [53]. Hydroxyapatite adhesion is higher on TNTs surfaces compared with non-anodized surfaces [54]. The mechanical interlocking between the hydroxyapatite coating and the nanotubular titanium oxide layer improves cell adhesion. Hydroxyapatite formation increases as thickness of oxide layer increases and it is higher on crystalline structure compared to amorphous structure [27]. In several studies osteoblast cells and mesenchymal stem cells behavior on TNTs have been investigated. The effect of other factors including nanotubes diameter, crystallinity and wettability is also verified as discussed in following sections.

\section{Osteoblast cells}

Behavior of osteoblasts cells on the $\mathrm{TiO}_{2}$ nanotubes is improved compared to the non-anodized titanium surface. Increase in surface roughness, increases hydrophilicity and surface energy and as a result improves bone-cell interaction. Experiments also show that filopodia of the osteoblasts grow into nanotube porosities and provides an integrated structure (figure 2.4) [53]. Effect of nanotube structure on attachment, growth and differentiation of human osteoblast is investigated by 3-(4,5-Dimethylthiazol-2-Yl)-2,5Diphenyltetrazolium Bromide (MTT) assay and Alkaline phosphatase (ALP) measurement in previous experiments. Results show that surface modification increases cell adhesion, cell proliferation and osteoblast expression. Cells seeded on nanotube structure show filamentous network structure and formation of nodules and increased extracellular matrix (ECM) [10]. The possibility of enhancing the $\mathrm{TiO}_{2}$ nanotubes bioactivity is verified by exposure to $\mathrm{NaOH}$ solution. The findings indicate that the sodium titanate nanostructure formed on the edge of the anodized $\mathrm{TiO}_{2}$ nanotubes can increase in vitro hydroxyapatite formation [48]. 


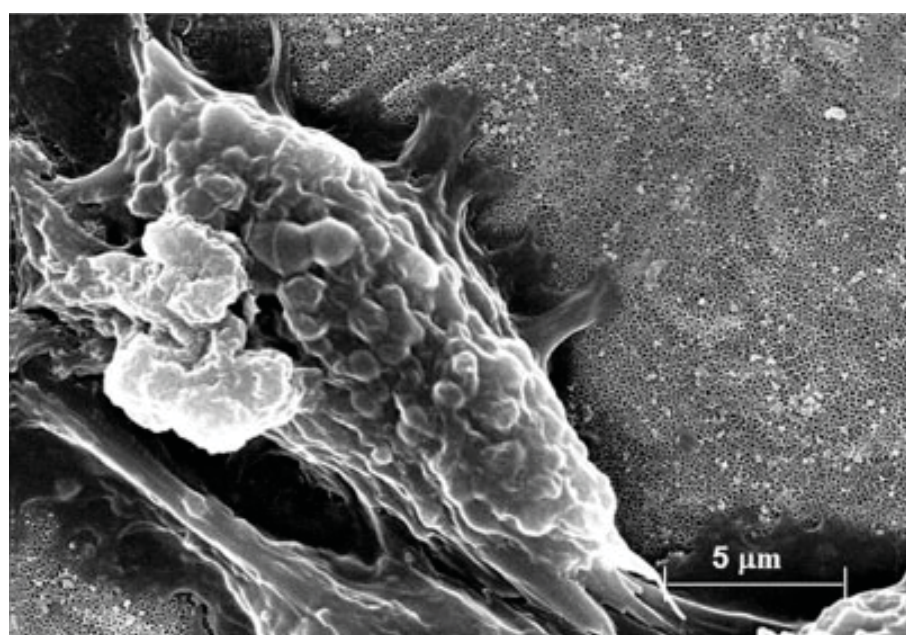

Figure 2.4 Human osteoblast cell attaches to nanotubular surface while filopodia penetrates into porosities as anchorage sites [10]. Reproduced with permission from John Wiley and Sons Inc.

\section{Mesenchymal stem cell}

Being derived from bone marrow, Mesenchymal Stem Cells ( MSCs) are pluripotent cells that have the potential to differentiate into different cell types including osteoblasts [55]. It is shown that nanotubes with size range between 15 to $30 \mathrm{~nm}$ provide proper substrate for MSC interaction. An increase of focal contact formation was observed on nanotubes smaller than $30 \mathrm{~nm}$ and increased cell proliferation and osteoblast differentiation was observed on $15 \mathrm{~nm}$ nanotubes. Also differentiation of hematopoietic stem cells (HSC) into osteoclasts increased in nanotubes below $30 \mathrm{~nm}$. Similar observations were found from differentiation of MSCs to osteoblasts [53].

Thus the diameter of nanotubes drastically affects cellular response. Previous studies conclude that stem cells dramatically respond to change in size of $\mathrm{TiO}_{2}$ nanotubes in range of 15 to $100 \mathrm{~nm}$. More importantly they suggest that small nanotubes having diameter less than $30 \mathrm{~nm}$, increase cell adhesion, proliferation, migration and integrin clustering/focal contact formation. These reactions tend to decline significantly with increasing pore size [49,56-59].

Experiments by Schumki et al. led to the conclusion that differentiation, protein aggregation, lamellipodia extension and filopodia extension was higher on smaller 
nanotubes [49,57-59]. However, a recent study concludes that differentiation, protein aggregation, lamellipodia extension and filipodia extension increases as nanotube diameter increases [56]. Authors of this study assume that on $100 \mathrm{~nm}$ nanotubes, hMSCs need to struggle to find $\mathrm{TiO}_{2}$ region where more protein aggregates have been deposited. Therefore they form more elongated shape and their filopodia is extended. These results are compatible with results of $\mathrm{McBeath}$ et al. who reported that decreasing cell density increases osteoblastic differentiation. These data are also compatible with the hypothesized concept that increasing physical stress increases stem cell differentiation.

Bauer et al. investigated the effect of change in dimension of nanotubes on MSCs response attachment and proliferation. They concluded that change in size is more effective on cell response compared to change in surface chemistry and length size [36].

\section{Chondrocyte}

Similar to osteoblasts, chondrocytes attachment on anodized $\mathrm{TiO}_{2}$ nanotubes increases compared with unanodized Ti. Nanotubular structure increases surface area and initial protein adsorption, therefore interaction and adhesion of chondrocyte is increased. Glycosaminoglycan secretion in the culture medium is reported to increase and chondrogenic markers such as aggrecan and collagen type II are also shown in higher level. Although the cells produced dense ECM fibrils, they retained their circular morphology $[34,60]$.

\section{Fibroblast and keratinocyte}

Biomaterials that are implanted as transcutaneous device interact with both the fibroblasts of dermal (internal) layer of the skin, and keratinocytes of the epidermal (external) layer. The responses of fibroblasts and keratinocytes on $\mathrm{TiO}_{2}$ nanotubes have been investigated to verify its potential for transcutaneous application. Studies indicate

that the nanotube topography provides a proper substrate for interaction of fibroblasts cells but not for keratinocytes cells $[61,62]$.

Compared to the smooth titanium substrate, adhesion of fibroblasts is increased on nanotubular surface while keratinocytes is decreased. Similarly MTT assays show 
increases of cell proliferation rate for fibroblasts but decreased of proliferation for keratinocytes on $\mathrm{TiO}_{2}$ nanotubes substrate compared with the smooth substrate. In addition, cytoskeleton reorganization improvement and membrane protein expressions were observed for fibroblasts cells while keratinocytes cells showed lack of cytoskeleton reorganization [61,62]. Indirect immunofluorescence staining characterizing was

performed for specific marker proteins to investigate cell proliferation. The results show an increase in specific marker expression of fibroblasts cells and decrease in specific marker of keratinocytes $[61,62]$.

\section{Endothelial and muscle cell}

$\mathrm{TiO}_{2}$ nanotube structure has the potential to be used as a vascular stent material. As a vascular stent device, a biomaterial interacts with smooth muscle cells and endothelial cells. Endothelial cell growth is enhanced on nanotubular morphology while MOVAS smooth muscle cell show little tendency to proliferate. Nanotubes maintain the differentiated state of muscle cells and their non-proliferative phenotype while they allow arranged changes in endothelial cell locomotion, cytoskeleton organization and cell-tocell communication [63].

In order to assess thrombogenicity nanotubular surface, the release of nitrogen oxide and endothelin-1 is investigated in presence of nanotubular structure. Nitrogen oxide causes vasodilatation and inhibits platelet aggregation while endothelin-1 causes vasoconstriction and enhances platelet aggregation. The results show that nitrogen oxide and endothelin-1 release are balanced in a way that nanotubular structure have antithrombotic effect [63].

Schmuki et al. assessed differentiation of mesenchymal cells to endothelial cells and smooth muscle cells on $\mathrm{TiO}_{2}$ nanotubes. In agreement with their previous studies, they concluded that $15 \mathrm{~nm}$ diameter maximizes differentiation of mesenchymal cells to endothelial cells and smooth muscle cells [64].

\subsubsection{Antibacterial effects}


Anodization of pure titanium and Ti6A14V alloy surfaces, results in decrease of bacterial attachment and biofilm formation compared to non-anodized surfaces in vitro and in vivo. Application of higher voltages leads to enhanced antibacterial effects. Treatment with high voltage also results in increased proliferation of osteoblasts and fibroblasts [65]. The most robust antibacterial response of TNT surface is reported to be achieved on $80 \mathrm{~nm}$ diameter nanotubes after heat treatment [66]. Antibacterial property of TNTs is enhanced following exposure to UV light illumination [67]. In addition, TNTs can be loaded with antibiotics in order to further reduce bacterial adhesion [68].

\subsubsection{Effect of anodization parameters}

\section{Effect of diameter}

Diameter of the nanotubes drastically affects cellular response. Biochemical response of cells on nanotubular surface is dependent on how they are stimulated mechanically by nanotubes. Diameter of nanotubes defines position of transmembrane integrins of attached cells. Integrins transmit the force to actin filaments and cause cytoskeletal tension and consequently cell morphology and signaling is affected [69]. Several studies have investigated the effect of variation of nanotubes diameters in the range of 15 to $100 \mathrm{~nm}$ on biological response. Although results of most studies show that adhesion of osteoblasts is higher on smaller nanotubes, contradictory results are reported for proliferation, ALP activity and other cell responses (Table 2.1). Yu et al. evaluated effect of change in diameter of anatase- $\mathrm{TiO}_{2}$ nanotube layers produced by anodization on adhesion, proliferation and differentiation of MC3T3-E1 preosteoblasts. The cell proliferation increased with increasing diameter of nanotubes. According to cell adhesion and ALP activity it was concluded that tubes with diameter of $20-70 \mathrm{~nm}$ provide better condition for integrin clustering [70]. Another study showed that greatest degree of cell adhesion and proliferation happens on $30 \mathrm{~nm} \mathrm{TiO}$ nanotubes and increasing the diameter up to $70-100 \mathrm{~nm}$ decreases proliferation and cells show elongated shape and higher ALP levels (figure 2.5) [34]. Effect of length of the nanotubes on cellular response is not explored as much as effect of tubes diameter. Tube length does not seem to affect the cell behavior [58]. 


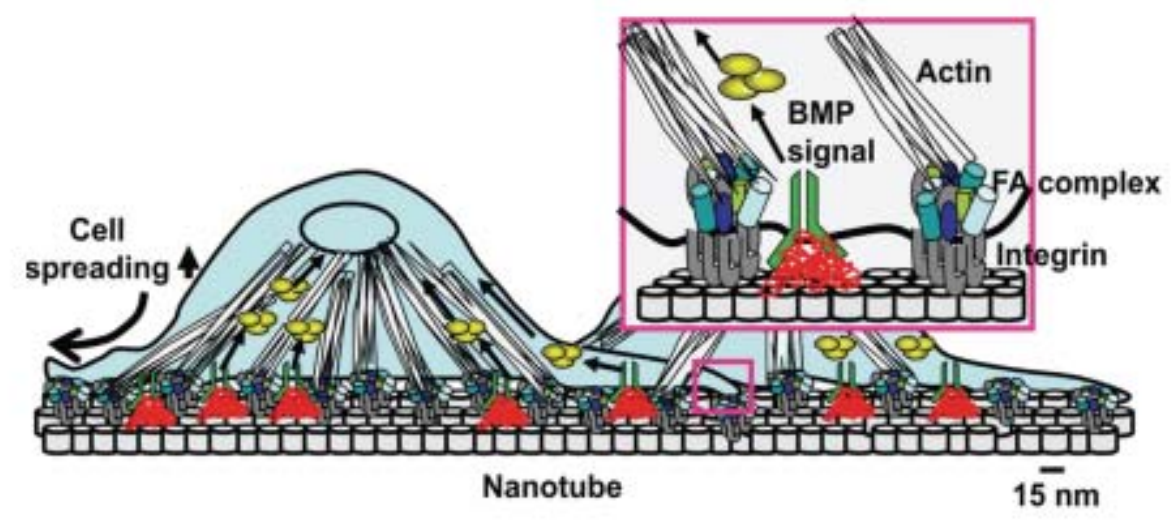

Figure 2.5 Clustering of integrins, formation of focal adhesions, MSCs spreading, actin polymerization, and osteogenic differentiation are increased on a nanotubular surface with $15 \mathrm{~nm}$ lateral spacing, in presence of BMP-2 signaling [59].

Reproduced with permission from John Wiley and Sons Inc.

Table 2.1 Effect of nanotube diameter on cellular behavior

\begin{tabular}{|c|c|c|c|c|c|}
\hline $\begin{array}{c}\text { Anodization } \\
\text { Condition }\end{array}$ & $\begin{array}{l}\text { Annealing } \\
\text { Conditions }\end{array}$ & Cell Type & $\begin{array}{c}\text { Cell Culture } \\
\text { Duration }\end{array}$ & Effect of TNT Size & Ref \\
\hline $\begin{array}{l}\text { Anode: Titanium foil } \\
\text { Cathode: Platinum } \\
\text { Electrolyte: } 1 \mathrm{M} \\
\mathrm{H}_{3} \mathrm{PO}_{4} \text { and } 0.5 \mathrm{wt} \% \\
\mathrm{HF} \\
\text { Duration: } 3 \mathrm{~h} \\
\text { Voltage: } 5 \text { to } 25 \mathrm{~V}\end{array}$ & $\begin{array}{l}450{ }^{\circ} \mathrm{C} \text { for } \\
3 \mathrm{~h}\end{array}$ & MC3T3-E1 & $\begin{array}{l}\text { 2h before Cell } \\
\text { adhesion. } 24,48, \text { and } \\
96 \mathrm{~h} \text { before MTT. 1, 2, } \\
\text { and } 3 \text { weeks for ALP } \\
\text { activity. } 3 \text { week before } \\
\text { Alizarin R-staining. }\end{array}$ & $\begin{array}{l}\text { Diameter of } 20-70 \\
\text { nm enhanced cell } \\
\text { adhesion, } \\
\text { alkaline phosphatase } \\
\text { activity, and } \\
\text { mineralization. The } \\
\text { proliferation } \\
\text { increased with } \\
\text { increasing tube } \\
\text { diameter from } 20 \text { to } \\
120 \mathrm{~nm} \text {. }\end{array}$ & {$[70]$} \\
\hline $\begin{array}{l}\text { Anode: Titanium foil } \\
\text { Cathode: Platinum } \\
\text { Electrolyte: } 1: 7 \\
\text { volumetric ratio of } \\
\text { acetic } \\
\text { acid to } 0.5 \% \mathrm{w} / \mathrm{v} \\
\text { hydrofluoric acid in } \\
\text { water } \\
\text { Duration: } 30 \mathrm{~min} \\
\text { Voltage: } 5,10,15 \\
\text { and } 20 \mathrm{~V}\end{array}$ & $\begin{array}{l}500^{\circ} \mathrm{C} \text { for } \\
2 \mathrm{~h}\end{array}$ & MC3T3-E1 & $\begin{array}{l}2 \mathrm{~h}, 12 \mathrm{~h}, 24 \mathrm{~h}, 48 \mathrm{~h}, 72 \mathrm{~h} \\
\text { and } 7 \mathrm{~d} \text { for cell } \\
\text { counting. } 24 \mathrm{~h} \text { and } 48 \mathrm{~h} \\
\text { for MTT and ALP. }\end{array}$ & $\begin{array}{l}30 \mathrm{~nm} \text { nanotubes } \\
\text { enhanced osteoblast } \\
\text { adhesion, while } 70- \\
100 \mathrm{~nm} \text { nanotubes } \\
\text { provide a lower } \\
\text { population } \\
\text { of cells with } \\
\text { elongated cellular } \\
\text { morphology and } \\
\text { enhanced alkaline } \\
\text { phosphatase levels. }\end{array}$ & [34] \\
\hline $\begin{array}{l}\text { Anode: Titanium } \\
\text { sheet } \\
\text { Cathode: Platinum } \\
\text { Electrolyte: } 0.5 \mathrm{wt} \% \\
\text { hydrofluoric acid and } \\
\text { acetic acid }\end{array}$ & $\begin{array}{l}500^{\circ} \mathrm{C} \text { for } \\
2 \mathrm{~h}\end{array}$ & hMSCs & $\begin{array}{l}2 \mathrm{~h}, 48 \mathrm{~h} \text { for Cell } \\
\text { adhesion. } 3 \text { weeks for } \\
\text { osteogenetic markers } \\
\text { microscopy. }\end{array}$ & $\begin{array}{l}30 \mathrm{~nm} \text { diameter } \\
\text { nanotubes enhanced } \\
\text { adhesion, while } \\
70 \text { to } 100 \mathrm{~nm} \\
\text { diameter nanotubes } \\
\text { show stem cell }\end{array}$ & {$[56]$} \\
\hline
\end{tabular}




\begin{tabular}{|c|c|c|c|c|}
\hline $\begin{array}{l}\text { volumetric ratio } 7: 1 \\
\text { acid in water } \\
\text { Duration: } 30 \mathrm{~min} \\
\text { Voltage: } 5,10,15 \\
\text { and } 20 \mathrm{~V}\end{array}$ & & & $\begin{array}{l}\text { elongation and } \\
\text { selective } \\
\text { differentiation into } \\
\text { osteoblast-like cells. }\end{array}$ & \\
\hline $\begin{array}{l}\text { Anode: Titanium } \\
\text { sheet } \\
\text { Cathode: Platinum } \\
\text { Electrolyte: } 1 \mathrm{M} \\
\mathrm{H}_{3} \mathrm{PO}_{4} \text { with addition } \\
\text { of } 0.3 \mathrm{wt} \% \mathrm{HF} \\
\text { Duration: } 1 \mathrm{~h} \\
\text { Voltage: } 1 \mathrm{~V} \text { up to } \\
20 \mathrm{~V}\end{array}$ & $\begin{array}{l}\text { hematopoietic } \\
\text { stem cells } \\
\text { (HSCs), } \\
\text { human } \\
\text { osteoblast- } \\
\text { like }\end{array}$ & $\begin{array}{l}2 \text { weeks } \\
\text { with differentiation } \\
\text { medium before } \\
\text { analysis by } \\
\text { Immunocytochemistry. } \\
3 \text { days before } \\
\text { osteoblast cell } \\
\text { counting. }\end{array}$ & $\begin{array}{l}\text { Diameters between } \\
15 \text { and } 100 \mathrm{~nm} \text { were } \\
\text { verified. } 15 \mathrm{~nm} \\
\text { supports HSCs } \\
\text { differentiation into } \\
\text { osteoclasts, adhesion } \\
\text { and osteoblast } \\
\text { proliferation. }\end{array}$ & [57] \\
\hline $\begin{array}{l}\text { Anode: Titanium foil } \\
\text { Cathode: Platinum } \\
\text { Electrolyte: } 1 \mathrm{M} \\
\mathrm{H}_{3} \mathrm{PO}_{4} \mathrm{with} \text { addition } \\
\text { of } 0.3 \mathrm{wt} \% \mathrm{HF} \\
\text { Voltage: } 1 \mathrm{~V} \text { up to } \\
20 \mathrm{~V}\end{array}$ & $\begin{array}{l}\text { Rat } \\
\text { mesenchymal } \\
\text { stem cells }\end{array}$ & $\begin{array}{l}2 \text { weeks before } \\
\text { analysis by } \\
\text { immunocytochemistry. } \\
3 \text { and } 6 \text { days before } \\
\text { cell counting. }\end{array}$ & $\begin{array}{l}\text { Diameter less than } \\
30 \mathrm{~nm} \text { with a } \\
\text { maximum at } 15 \mathrm{~nm} \\
\text { enhanced integrin } \\
\text { clustering/focal } \\
\text { contact } \\
\text { formation and } \\
\text { cellular activities. }\end{array}$ & [49] \\
\hline $\begin{array}{l}\text { Anode: Zirconium } \\
\text { and titanium foils } \\
\text { Cathode: Platinum } \\
\text { Electrolyte: For } \mathrm{Zr}, 1 \\
\mathrm{M}\left(\mathrm{NH}_{4}\right)_{2} \mathrm{SO}_{4} \text { with } \\
\text { the addition of } 0.15 \\
\mathrm{M} \mathrm{NH}_{4} \mathrm{~F} \text {. For Ti, } 1 \\
\mathrm{M} \mathrm{H}_{3} \mathrm{PO}_{4} \text { with the } \\
\text { addition of } 0.125 \mathrm{M} \\
\mathrm{HF}\end{array}$ & $\begin{array}{l}\text { Rat } \\
\text { mesenchymal } \\
\text { stem cells }\end{array}$ & $\begin{array}{l}1 \text { day before cell } \\
\text { adhesion and } 3 \text { days } \\
\text { before cell } \\
\text { proliferation. }\end{array}$ & $\begin{array}{l}\text { Both materials } \\
\text { provide enhanced } \\
\text { cell adhesion and } \\
\text { proliferation with } \\
\text { nanotube diameters } \\
\text { of } 15-30 \mathrm{~nm} \text {. }\end{array}$ & [58] \\
\hline $\begin{array}{l}\text { Anode: Titanium } \\
\text { foils } \\
\text { Cathode: platinum } \\
\text { Electrolyte: } 1 \mathrm{~m} \\
\mathrm{H}_{3} \mathrm{PO}_{4} \text { with addition } \\
\text { of } 0.12 \mathrm{M} \mathrm{HF} \\
\text { Voltage: } 1 \text { and } 20 \mathrm{~V}\end{array}$ & $\begin{array}{l}\text { mesenchymal } \\
\text { stem cells }\end{array}$ & $\begin{array}{l}24 \mathrm{~h} \text { for cell counting. } \\
2 \text { weeks in } \\
\text { differentiation medium } \\
\text { before } \\
\text { immunocytochemistry. }\end{array}$ & $\begin{array}{l}\text { Differentiation is } \\
\text { enhanced on } 15 \mathrm{~nm} \\
\text { but not on } 100 \mathrm{~nm} \\
\text { BMP-2-coated } \\
\text { nanotubes. }\end{array}$ & [59] \\
\hline
\end{tabular}




\section{Effect of crystallinity}

Crystallinity, the degree of structural order, is a surface factor that affects cell behavior. Under most anodization conditions as formed $\mathrm{TiO}_{2}$ nanotube have amorphous structure. Annealing at $450{ }^{\circ} \mathrm{C}$ and $600{ }^{\circ} \mathrm{C}$ for $3 \mathrm{~h}$ leads to formation of different crystalline phases of anatase and rutile respectively [71-73]. Relative amount of anatase formation is higher for the samples anodized with a higher voltage compared to the samples anodized at lower voltage [65]. Crystallized phase of substrate increases hydrophilicity [74]; and consequently, enhances desirable responses of cells cultured on it [75]. MC3T3-E1 preosteoblasts activity and tendency to spread increases as nanotubes amorphous structure changes to pure anatase and is maximized when pure anatase transforms to anatase-rutile. Not only cell proliferation increases with increasing annealing temperature but also apatite mineralization and corrosion-resistance is maximized on rutile structure (figure 2.6) $[9,76]$. Highest amount of filopodia extension occurs on anatase structure [76] while filopodia formation is maximized on anatase [9]. Cell adhesion increases as amount of present fluoride increases. Annealing nanotubes decreases the amount of fluoride and cell numbers [64].

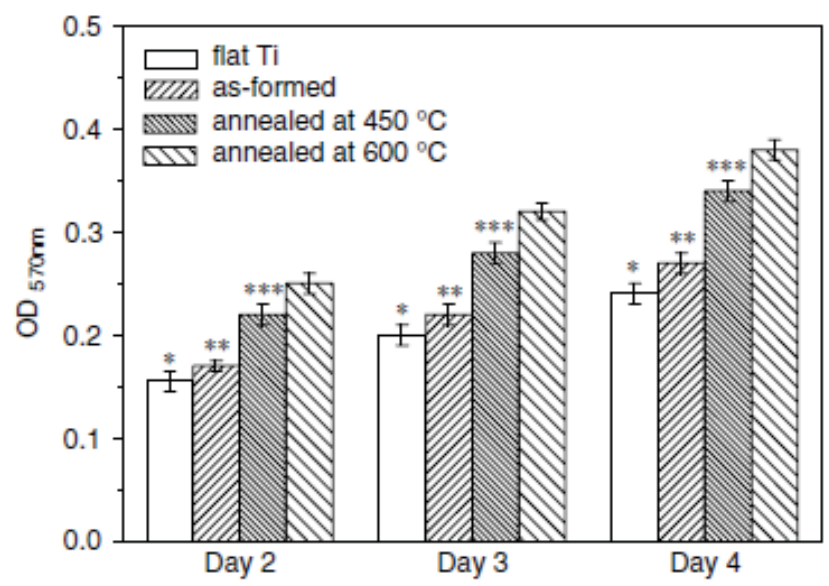

Figure 2.6 MTT assay shows effect of surface crystallinity on differentiation behavior of osteoblast cells [76].

Reproduced with permission from John Wiley and Sons Inc.

Transformation from amorphous to anatase structure slightly increases Yang modulus and hardness while transformation from anatase to rutile sharply increases 
theses mechanical properties. Since high hardness and low Yang modulus is desirable for biomedical application, an anatase/rutile structure is suggested to be utilized to optimize mechanical properties [76]. Yang modulus is also influenced by diameter and wall thickness of nanotubes [22].

\section{Effect of roughness}

Roughness is increased on nanotube structure compared to smooth titanium as measured by AFM [9]. Effect of surface topography is shown to be higher compared to crystallinity and surface chemistry [64]. Increasing voltage of anodization slightly increases surface roughness and biological response is affected to some extent by surface roughness variance in nano scale [65].

Roughened surface of titanium in micro scale, compared to flat surface, is anticipated to provide mechanical interlocking for long time. In addition cell functions such as cell adhesion and gene expression are promoted after acid etching [77]. The micro-nano scale structure produced by anodization of roughened titanium surface mimics structure of natural bone and has shown to increase hydroxyapatite formation and protein adsorption [78].

\section{Effect of wettability}

Wettability is another factor that affects osteoblast behavior. Water contact angle is decreased after anodization [65]. Surface of titanium becomes hydrophilic after anodization and hydrophilicity further increases when anodized surface in annealed. Interestingly, nanotubular surface loses part of its hyrophilicity when it is exposed to air for a period of three months. Ambient atmosphere affects wettability probably through alkane contamination and organic contaminants [79]. Super-hydrophilic $\mathrm{TiO}_{2}$ nanotube become hydrophobic when coated with a monolayer of octadecylphosphonic acid. Comparison of mesenchymal stem cells adhesion, spreading and growth on the unmodified nanotubes with modified nanotubes shows that coating diminishes effect of tube diameter and hydrophobicity causes decrease of proliferation [80]. 

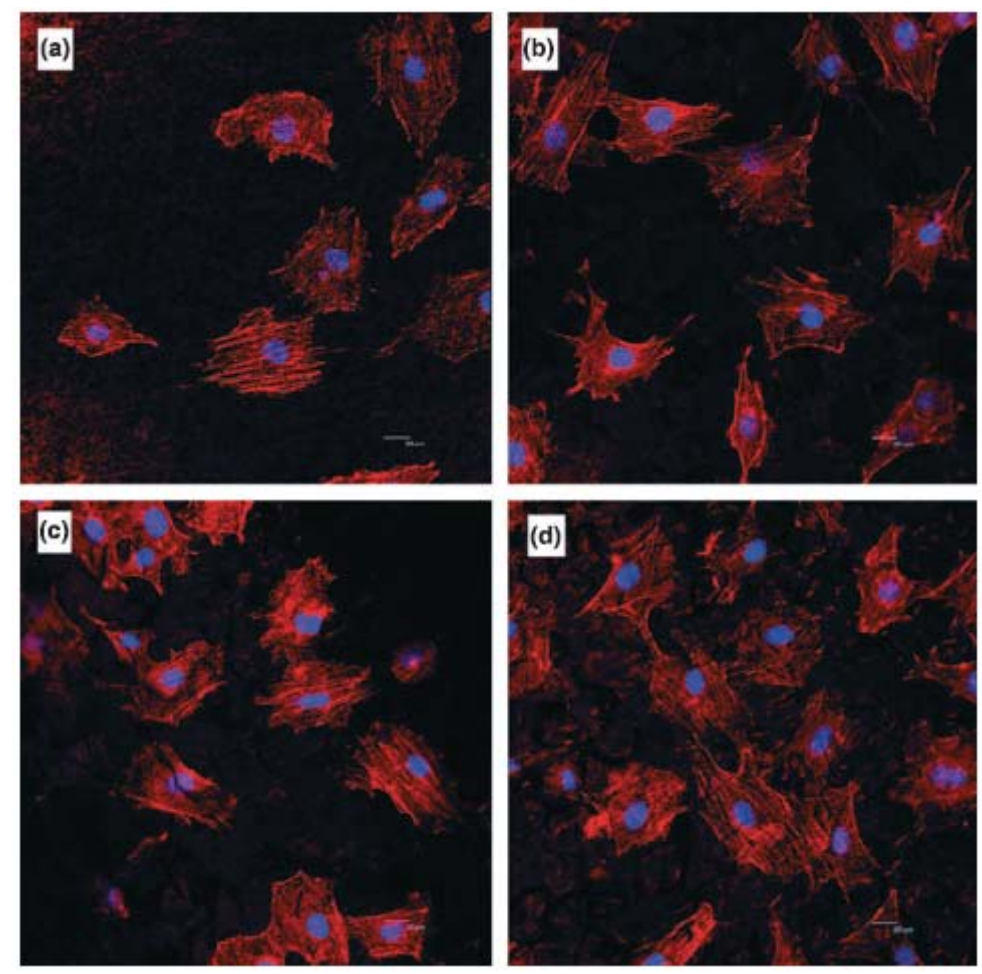

Figure 2.7 Staining actin cytoskeleton of MC3T3-E1 osteoblast on: (a) smooth surface, (b) non-annealed nanotubular surface, nanotubular surfaces annealed at (c) $450{ }^{\circ} \mathrm{C}$ and

(d) $550^{\circ} \mathrm{C}$. Compared with the smooth and the non-annealed nanotubular surfaces, annealed surfaces show higher regular arrangement [9].

Reproduced with permission from John Wiley and Sons Inc.

\subsection{Controlling TNTs dimensions}

TNT dimensions can be controlled by optimizing different parameters including electrolyte composition, electrolyte $\mathrm{pH}$, type of electrolyte, voltage magnitude and anodization duration [34,81-83]. Also agitation speed of electrolyte, temperature and the ratio of cathode-to-anode surface area affect morphology of TNTs [84]. Diameter of TNTs increases as either applied voltage or anodization duration increases (figure 2.8). Length of TNTs can be increased decreasing acidity and fluoride concentration [44]. As $\mathrm{pH}$ is increased the time taken for nanotube formation increases; therefore, fabricated nanotubes are longer [85]. Anodization duration slightly affects diameter but length of 
TNTs is gradually increased over time before a steady state condition is established under which length remains constant (Table 2.2). As fluoride ion concentration increases in electrolyte solution, dissolution rate of the oxide wall is enhanced and the wall thickness is decreased [86]. Length of the nanotube fabricated at different conditions varies from $200 \mathrm{~nm}$ to $360 \mu \mathrm{m}$, the wall thickness of the nanotube changes from $5 \mathrm{~nm}$ to $34 \mathrm{~nm}$, and tube diameter varies from $12 \mathrm{~nm}$ to $180 \mathrm{~nm}$ [87].

$(4 \mathrm{hrs})$

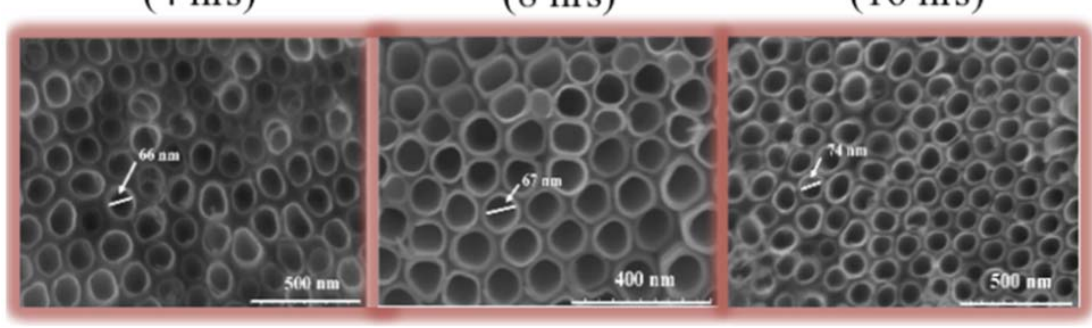

Figure 2.8 SEM images of TNT fabricated during varying durations [88].

Table 2.2 Effect of anodization time and voltage on TNT length.

\begin{tabular}{|c|c|c|c|}
\hline $\mathbf{V}$ Time & $\begin{array}{c}\mathbf{4 h} \\
(\boldsymbol{\mu} \mathbf{m})\end{array}$ & $\begin{array}{c}\mathbf{8 h} \\
(\boldsymbol{\mu} \mathbf{m})\end{array}$ & $\begin{array}{c}\mathbf{1 6 h} \\
(\boldsymbol{\mu} \mathbf{m})\end{array}$ \\
\hline $\mathbf{2 0} \mathbf{V}$ & 0.589 & 1.07 & 1.39 \\
\hline $\mathbf{4 0} \mathbf{V}$ & 1.443 & 4.53 & 6.11 \\
\hline $\mathbf{6 0} \mathbf{V}$ & 5.493 & 6.75 & 10.08 \\
\hline
\end{tabular}

\subsubsection{Electrolyte composition}

The various electrolytes used for nanotubes fabrication are categorized into three groups: (i) acidic aqueous solution containing fluoride ion, (ii) buffered aqueous solution and (iii) non-aqueous solution containing fluoride ion and in some cases a low amount of water [6]. When non-aqueous electrolyte (organic electrolyte) is employed, longer nanotubes are formed over a longer period of time compared to aqueous electrolyte. This is because an organic electrolyte contains less amount of oxygen compared with an aqueous solution and chemical dissolution of oxide is dependent on the water content [89]. Length of TNTs fabricated in ethylene glycol is maximized and reaches up to 45 
$\mu \mathrm{m}$ when solution contains $2 \mathrm{vol} \% \mathrm{H}_{2} \mathrm{O}, 0.2 \mathrm{wt} \% \mathrm{NH}_{4} \mathrm{~F}$ and $60 \mathrm{~V}$ is applied for $18 \mathrm{~h}$ [90]. Variation in amounts of $\mathrm{H}_{2} \mathrm{O}$ and $\mathrm{NH}_{4} \mathrm{~F}$ caused decrease of length.

Increasing anodization duration up to $18 \mathrm{~h}$ elongates tubes but no significant increase in length is observed after $18 \mathrm{~h}$. In another study the electrolyte consisted of $98 \mathrm{vol} \%$ ethylene glycol, 2 vol\% deionized water and $0.25 \mathrm{wt} \% \mathrm{NH}_{4} \mathrm{HF}_{2}$. Anodization was performed for 8,18 and $30 \mathrm{~h}$ at $70 \mathrm{~V}$. The nanotube length is maximized at $18 \mathrm{~h}$ to $6.5 \mu \mathrm{m}$ [91]. Schmuki et al. investigated electrolytes of ethylene glycol containing less than 0.2 $\mathrm{wt} \% \mathrm{H}_{2} \mathrm{O}$ and $\mathrm{HF}$. They concluded that length of nanotubes is maximized at $120 \mathrm{~V}, 0.2$ $\mathrm{mol} / \mathrm{l} \mathrm{HF}$ and $15 \mathrm{~h}$. Increasing voltage or HF concentration leads to electro-polishing. In the above mentioned conditions tube length of $261 \mu \mathrm{m}$ with an internal diameter of 70 $\mathrm{nm}$ and external diameter of $160 \mathrm{~nm}$ is obtained [92].

\subsection{Nanotube development on zirconium surface}

\subsubsection{Zirconium versus titanium}

Although pure zirconium is potential for providing proper interaction with cells, it has not been explored as much as titanium. Zirconium has enormous potential applications in the field of biomedical implants [93]. Biocompatibility and corrosion resistance of specific zirconium alloys are proper as well as titanium alloys and the mechanical properties of zirconium alloys compared to Ti6Al4V alloy have been observed to be higher [94]. Titanium alloys that contain zirconium show better tensile and fatigue strength than pure titanium [95]. When titanium is exposed to body fluids such as saliva, it undergoes electrochemical corrosion. Consequently, ions are released from the surface of biomaterial. In contrary, zirconium does not show undesirable electrochemical characteristics. Also zirconium color is similar to tooth while titanium has a gray shine [96].

In a comparative animal study, zirconium implants demonstrated identical osseointegration as titanium implants. In addition, no significant difference was observed between healing of the tissues interacting with the materials [96].

\subsection{2 $\mathrm{ZrO}_{2}$ nanotube formation}


Since presence of nanostructure on implant surface is shown to enhance desirable cellular response, fabrication of $\mathrm{ZrO}_{2}$ nanotubes by anodization is studied, and the influence of various electrochemical factors have been evaluated including potential of power source and its sweep rate, electrolyte composition and anodization time [93]. Preanodization of zirconium is reported to be beneficent to form highly ordered $\mathrm{ZrO}_{2}$ nanotubes. Also $\mathrm{ZrO}_{2}$ nanotube structures that are fabricated on electropolished zirconium show more uniformity [93]. When organic electrolytes are employed for anodization, the nanostructure formed is thicker, more regular and less wavy [97-100].

Microstructure of substrate influences anodic oxidizations and eventually affects fabrication of $\mathrm{ZrO}_{2}$ nanotubes. Following surface mechanical attrition treatment, commercially pure zirconium has nanocrystallized surface layers with high density of grain boundaries compared with non-treated zirconium. Nanocrystallized zirconium is beneficent to the formation of $\mathrm{ZrO}_{2}$ nanotubes and grain boundaries are effective in accelerating reaction rate. $\mathrm{ZrO}_{2}$ nanotube layer formed on the treated zirconium is considerably thicker than that formed on the non-treated zirconium. Thickness increases also with increase in anodization duration and follows a parabolic function [93].

The SEM images show that nanotubes are gradually formed on the flat surface similar to formation of $\mathrm{TiO}_{2}$ nanotubes. Self-organization is suggested to be product of competition between growing pores and elsewhere is suggested to be result of local surface perturbations. Localized dissolution of $\mathrm{ZrO}_{2}$ causes formation of pores and reduction of oxide layer thickness. As a result, the electrical field intensity is increased at the base of pore and creation of new oxide is induced. In addition, similar to growth mechanism of nanotube on titanium, $\mathrm{ZrO}_{2}$ nanotube formation in electrolytes that contain fluoride is the outcome of a competition between oxide formation and its chemical dissolution by fluoride ions [93].

Different elements that are present in Ti15Nb4Ta4Zr (TNTZ) alloy have different electrochemical oxidation rates. Therefore reaction rate of TNTZ anodization depends on its composition. Different sizes of self-organized nanotubes are formed on the surface. Eight tubes with small diameter surround a tube of larger diameter [101]. Anodization of Ti28Zr8Nb alloy leads to formation of tubes with $98 \mathrm{~nm}$ diameter that surround a larger tube of $175 \mathrm{~nm}$ diameter [102]. Nanotubes that formed on TiZr alloy exhibit uniform 
arrays; however, as the zirconium content was increased the diameter of the tubes decreased and the length increased [103].

\subsection{Cellular behavior on zirconium nanotube}

For the osteoblasts cells cultured on $\mathrm{ZrO}_{2}$ nanotubes the initial adhesion, spreading, growth, functionality in terms of alkaline phosphatase activity and the formation of extracellular matrix is reported to considerably improve as compared with smooth zirconium surface. The cells attached on the nanotube surface demonstrated a high cytoskeleton organization, which was lacking on the flat zirconium [94]. Mesenchymal stem cells respond identical to $\mathrm{ZrO}_{2}$ nanotubes, $\mathrm{TiO}_{2}$ nanotubes and $\mathrm{AuPd}$-coated $\mathrm{TiO}_{2}$ nanotubes. Cell response is chiefly based on nano-topographical features rather than a certain surface chemistry related to $\mathrm{TiO}_{2}[94]$.

\subsection{Bone Implant Contact (BIC) and Bone Mineral Deposition (BMD)}

Frandsen et al. compared osseointegration of titanium-zirconium (TiZr) with pure titanium and Ti6A14V alloy. Although the formation of new bone inside the implant grooves increased over time regardless of the implant material; however, the amount of bone to implant contact (BIC) was shown to be a function of the implant material. For $\mathrm{TiZr}$ and pure titanium implants, the BIC increased gradually but for Ti6Al4V implants the BIC peaked after 2 weeks followed by a decline after 8 weeks. On surface of Ti6A14V implants, considerably more coverage by multinucleated giant cells was observed. Briefly, TiZr and pure titanium implants showed earlier osseointegration compared with Ti6Al4V implants. Maturation of bone marrow next to Ti6A14V implants was observed to be less advanced compared to TiZr and pure titanium implants [94]. Lack of considerable difference between BIC of titanium and zirconium were also detected in another in vivo study while BIC was affected by roughness. A considerably higher BIC was observed for zirconium implants with regular roughness compared with

low and high roughness implants [96]. In addition, bone mineral density (BMD) around TNTZ alloy is observed to be similar to Ti6Al4V [104]. 


\subsection{Drug loading on nanotubes}

Cellular response can further be improved by application of drug. Local drug delivery is developed in order to overcome systemic side effects and its delivery deficiencies. Many drugs are not effectively delivered via systemic routes. For example when antibiotics such as Neomycin and Gentamicin [105] are taken orally, they are absorbed from the small intestine and inactivated. Bone Morphogenic Protein 2 (BMP-2), as an osteogenic factor, is often delivered intravenously or topically. However, avascular tissue formed after surgery inhibits delivery of drug to implant-tissue interface. Increasing systemic doses to overcome this disadvantage leads to organ toxicity [87].

Drug loading on TNTs is not well-explored yet although drug loading on carbon nanotubes is reported in several studies to be promising for cancer therapy. Carbon nanotubes need to be prefunctionalized through oxidation and pegylation to become water soluble and biocompatible. Then further functionalization also is needed to attach the drug onto carbon nanotubes and target them toward cancer tissue. After being taken

up by the cell, carbon nanotubes release their cargo and later they are eliminated from the body [106-112]. However, application of carbon nanotubes is restricted since they show toxicity and inflammation $[113,114]$.

Not only TNTs are potential to be loaded with drug and antibacterial agents, but also they are biocompatible and hydrophilic in contrast to carbon nanotubes. Although local drug delivery provides targeted release of drug, control of drug release over time remains to be a challenge. Preventing sudden release of drug after implantation avoids denaturation of drug and enhances its efficiency [115]. Formation of nanotubular structure on machined surfaces increases the amount of loaded BMP-2 and prolongs drug release [116]. TNTs loaded with BMP-2 were coated with multi-layers of gelatin and chitosan to further retard release [115]. Although controlled release of drug from TNT was successfully achieved through this technique, the polymeric coating that is used in this method prevents cellular interaction with surface nanostructure. When titania nanotube arrays are loaded with polymer micelles as drug carriers [117], surface nanostructure can induce cellular response. Since degradation of the polymer may induce 
inflammation; it is preferable to avoid its application and change the nanotube dimension for optimizing drug release. Anodization of Ti4Zr22 Nb2Sn at different potentials, concentration of $\mathrm{NH}_{4} \mathrm{~F}$ and anodization time shows that longer nanotubes prolong drug release [118]. Release of the antibiotic from elongated TNT formed in an electrolyte based on ethylene glycol is reported to be longer in comparison to release from shorter TNT that are formed in an aqueous solution [91]. The surface of titania nanotubes has a small negative charge due to presence of terminal hydroxyl groups. Therefore positively charged drugs are released slower compared to negatively charged agents [87]. Osteoblast response is also improved when nanotubes are loaded with antibiotics [119].

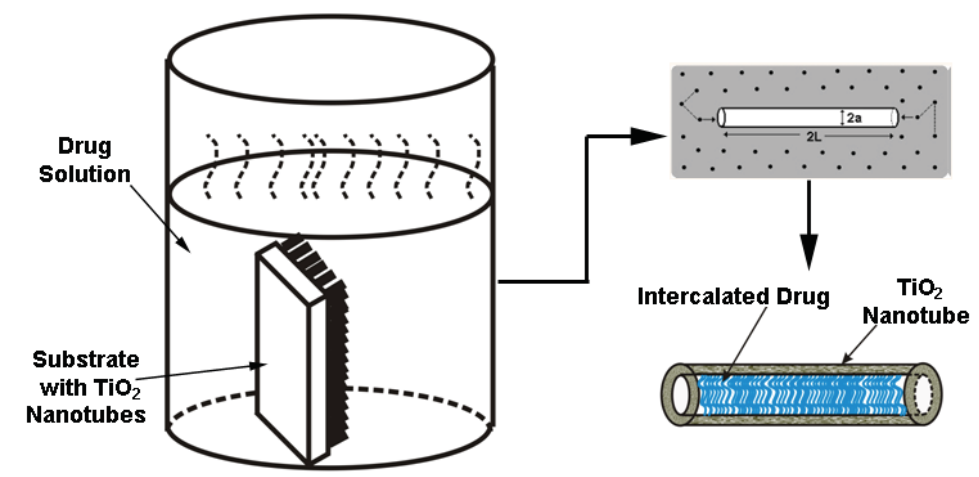

Figure 2.9 Intercalation of drug inside the nanotubes.

Reproduced by permission of The Royal Society of Chemistry [41].

\subsection{Summary}

Since titanium and zirconium provide several benefits compared to other biometals, they are widely used as a biomaterial for fabrication of bone and orthopedic implants. Desirable characteristics of titanium and zirconium alloys include biocompatibility, corrosion resistance and proper mechanical properties. Biocompatibility and corrosion resistance of specific zirconium alloys are as well as titanium alloys and the mechanical properties of zirconium alloys are higher than Ti alloys. Despite all these benefits, metal implants occasionally become loose or infectious after surgery which eventually results in implant failure. In order to overcome this problem, nanotechnology is used to modify the surface and increase osseointegration. Specifically, fabrication of $\mathrm{TiO}_{2}$ nanotubes 
through anodization technique on surface of titanium has shown great potential to promote desirable cellular behavior such as adhesion, proliferation and differentiation. In addition, hydroxyapatite mineralization is increased and bacterial adhesion is decreased on nanotubular surfaces compared with conventional smooth surfaces. Recent studies have successfully optimized properties of nanotubular surfaces to further increase osseointegration. In summary, it is concluded that nanotubes dimensions, heat treatment and drug loading can individually dictates cellar fate.

\section{Acknowledgments}

The authors would like to thank Prof. Craig Friedrich, MTU, Multi-Scale Technologies Institute for all of his contributions. The author would like to acknowledge the support offered by Dr. William Hendrickson at University of Illinois at Chicago. 


\title{
Chapter 3
}

\section{Fabrication of Anti-Aging TiO2 Nanotubes on Biomedical Ti Alloys*}

\author{
Azhang Hamlekhan ${ }^{1}$, Arman Butt ${ }^{2}$, Sweetu Patel ${ }^{2}$, Dmitry Royhman ${ }^{3}$, Christos \\ Takoudis $^{2,4}$, Cortino Sukotjo ${ }^{3}$, Judy Yuan ${ }^{3}$, Gregory Jursich ${ }^{4}$, Mathew T. Mathew ${ }^{5}$, \\ William Hendrickson ${ }^{6}$, Amarjit Virdi ${ }^{7}$, Tolou Shokuhfar ${ }^{1,8,9,10}$ \\ ${ }^{1}$ Department of Mechanical Engineering-Engineering Mechanics, Multi-Scale Technologies Institute, \\ Michigan Technological University, Houghton, MI, USA \\ ${ }^{2}$ Department of Bioengineering, University of Illinois at Chicago, Chicago, IL, USA \\ ${ }^{3}$ Department of Restorative Dentistry, College of Dentistry, University of Illinois at Chicago, \\ Chicago, IL, USA \\ ${ }^{4}$ Department of Chemical Engineering, University of Illinois at Chicago, Chicago, IL, USA \\ ${ }^{5}$ Department of Orthopedics, Rush University Medical Center, Chicago, IL, USA \\ ${ }^{6}$ Research Resources Center, University of Illinois at Chicago, Chicago, IL, USA \\ ${ }^{7}$ Department of Anatomy and Cell Biology, Orthopedic Surgery, Rush University, Chicago, IL, USA \\ ${ }^{8}$ Department of Mechanical and Industrial Engineering, University of Illinois at Chicago, Chicago, \\ IL, USA \\ ${ }^{9}$ Department of Physics, University of Illinois at Chicago, Chicago, IL, USA \\ ${ }^{10}$ Corresponding author, tshokuhf@mtu.edu
}

\footnotetext{
*The material contained in this chapter was previously published in the open access journal of PLOS ONE. As the first author of this publication, I have done the fabrication of nanotubes, FESEM, EDX, literature review and writing the manuscript.
} 


\begin{abstract}
The primary objective of this study was to fabricate a $\mathrm{TiO}_{2}$ nanotubular surface, which could maintain hydrophilicity over time (resist aging). In order to achieve nonaging hydrophilic surfaces, anodization and annealing conditions were optimized. This is the first study to show that anodization and annealing condition affect the stability of surface hydrophilicity. Our results indicate that maintenance of hydrophilicity of the obtained $\mathrm{TiO}_{2}$ nanotubes was affected by anodization voltage and annealing temperature. Annealing sharply decreased the water contact angle (WCA) of the as-synthesized $\mathrm{TiO}_{2}$ nanotubular surface, which was correlated to improved hydrophilicity. $\mathrm{TiO}_{2}$ nanotubular surfaces are transformed to hydrophilic surfaces after annealing, regardless of annealing and anodization conditions; however, WCA measurements during aging demonstrate that surface hydrophilicity of non-anodized and $20 \mathrm{~V}$ anodized samples decreased after only 11 days of aging, while the $60 \mathrm{~V}$ anodized samples maintained their hydrophilicity over the same time period. The nanotubes obtained by $60 \mathrm{~V}$ anodization followed by $600{ }^{\circ} \mathrm{C}$ annealing maintained their hydrophilicity significantly longer than nanotubes which were obtained by $60 \mathrm{~V}$ anodization followed by $300{ }^{\circ} \mathrm{C}$ annealing.
\end{abstract}

Keywords: Anodization, Sandblasting, Wettability, FTIR, SEM, Roughness, Anatase and Rutile

\title{
3.1 Introduction
}

Different categories of biomaterials have been employed to repair bone loss injuries. Among various biomaterials used in implant materials in orthopedics and dentistry [3], titanium and its alloys have recently attracted attention because of several advantages [6]. Compared with other biometals used as implants, titanium and its alloys provide biocompatibility in terms of low ion release [7], excellent corrosion resistance [120], great mechanical properties in terms of high hardness, low elastic modulus and low density $[9-11,13,121,122]$. The biocompatibility of titanium is a result of the presence of surface native oxide layer $\left(\mathrm{TiO}_{2}\right.$; titania; passive film) of $2-5 \mathrm{~nm}$ thickness which is naturally formed as titanium is exposed to air. This native oxide layer protects 
the bulk material from corrosion [15] and makes it bioinert [16]. Despite their bioinertness, titanium implants are sometimes encapsulated by fibrous tissue in vivo and show lack of osseointegration which can lead to infection and implant failure [123]. In addition, low $\mathrm{pH}$ and presence of lipopolysaccharide in saliva enhances corrosion rate of titanium dental implants [18]. In order to develop bioactivity and osseointegration, various surface modifications have been performed including hydroxyapatite (HA) and calcium phosphate coatings [30]; however these coatings could be delaminated at their interface with Ti because of difference in mechanical moduli [31].

To improve implant viability, nanotechnology holds certain advantages via modification of implant surfaces [51]. Recently, anodization techniques have been employed for formation of $\mathrm{TiO}_{2}$ nanotubes on titanium surfaces [19-21]. The anodized nanotubular surface shows promise for biomedical application due to increased osteoblast cell adhesion and function [19-21], increased growth of hydroxyapatite [27,28], and improved cellular behavior and tissue integration [29]. Compared with a flat surface (low surface roughness), a nanostructured surface provides more surface area for protein adsorption and as a result cellular interaction is enhanced [33]. Due to their integration with bulk substrate, $\mathrm{TiO}_{2}$ nanotubes do not suffer from delamination [34] and improve osseointegration of the implant.

Formation of $\mathrm{TiO}_{2}$ nanotubes on $\mathrm{Ti}$ substrate has been shown to decrease water contact angle (WCA) [65]. Reduction of WCA is significantly desirable because low WCA is correlated with enhanced bone cell interaction with the surface after implantation [53]. The initial interaction between implant surface and its physiological environment can play a key role in preventing implant failure. It is known that biomaterials are immediately covered with proteins from blood and interstitial fluids following exposure to physiologic environment [124]. When a biomaterial is exposed to in vitro or in vivo conditions, proteins that are present in the cell culture media or fluids of the body adsorb on its surface in less than 1s. Then, adsorbed protein functional groups (ligands) bond with cell surface receptors (integrins) [125]. Specifically, fibronectin and vitronectin are absorbed on the surface and form an intermediate layer that promotes cell adhesion $[126,127]$. Steele et al. showed that amount of protein adsorption is higher on hydrophilic surface compared with hydrophobic surface [128]. Other studies report that favorable 
response of cells is increased on hydrophilic surface compared with hydrophobic surface [129-132]. Surface modification using anodization has shown strong impact on improving hydrophilicity and cell interaction. Desirable cellular response of various cell lines is increased on nanotubular surfaces compared to flat machined surfaces. Such enhancement is due to an increase of surface area, which results in expansion of available area for cell-substrate interaction, surface energy, hydrophilicity, protein adsorption and consequently cell adhesion $[9,34,48,49,133]$.

Crystallinity is another factor that affects cellular behavior [134]. Under most anodization conditions, as-formed $\mathrm{TiO}_{2}$ nanotube structure is amorphous after anodization. Annealing at 450 and $600{ }^{\circ} \mathrm{C}$ for $3 \mathrm{~h}$ leads to formation of crystalline phases of anatase and rutile respectively. The relative amount of anatase formation is higher for the samples anodized with a higher voltage compared to the samples anodized at a lower voltage [65]. Crystallization of as formed $\mathrm{TiO}_{2}$ nanotubes further enhances desirable response of cells. MC3T3-E1 preosteoblast activity and tendency to spread increases as nanotube structure changes from amorphous to pure anatase, and is maximized when pure anatase transforms to an anatase-rutile mixture. Cell proliferation increases with increasing annealing temperature and apatite mineralization and corrosion resistance is maximized on rutile structure [9,76]; however, the highest amount of filopodia formation and extension occurs on anatase structure $[9,76]$.

Similar to other tissues of body, natural bone structure possesses nano, micro, and macro scale features. In the current study, hierarchical micro-nano scale topography of titania $\left(\mathrm{TiO}_{2}\right)$ is formed on the substrate via acid-etching and anodization respectively, in order to mimic natural bone morphology. In addition, cell functions, such as cell adhesion and gene expression, are promoted on acid-etched surfaces [135] and micro-nano scale structure of titanium surface increases hydroxyapatite formation and protein adsorption [136]. Although it is expected that a roughened surface on titanium and its alloys provides long term mechanical interlocking ability, it has not been explored to the extent of polished surfaces.

Previous studies have shown that hydrophobic Ti surfaces become hydrophilic after anodization, and hydrophilicity is further increased as the anodized surface is annealed [137]. Interestingly, the nanotubular surface loses part of its hydrophilicity after 
aging in air over a period of three months [137]. Considering the correlation between WCA and cellular response on bone implants, the aim of this study was to design hydrophilic surfaces that are able to maintain their low WCA. We hypothesized that optimized annealing and anodization parameters would improve hydrophilicity and minimize aging effect. Our results suggest that surface structure affects hydrophilicity and aging effect. Consequently, it is expected that such surfaces will maintain protein adsorption, cell adhesion, and osseointegration benefits of $\mathrm{TiO}_{2}$ nanotubes even after aging.

\subsection{Methods}

Fabrication of $\mathrm{TiO}_{2}$ nanotubes:

In this study Ti-6Al-4V alloy (Ti-V) disks (McMaster-Carr) with $15 \mathrm{~mm}$ diameter and $1 \mathrm{~mm}$ thickness were used. Disks were sandblasted with alumina powder (Trinity Tool Company) and acid-etched with a 1:1 ratio of sulfuric acid (Fisher) and hydrogen peroxide (30\% Fisher) for $2 \mathrm{~h}$. Samples were then washed with DI-water, sonicated in acetone for 30 minutes and dried in air.

In order to perform anodization, samples were connected to a voltage source (Keithley 2400 SourceMeter) as the working electrode and copper rod was used as the counter electrode. Both electrodes were immersed in electrolyte mixture of ethylene glycol (Fisher), $0.3 \mathrm{wt} \% \mathrm{NH}_{4} \mathrm{~F}$ (Fisher) and $2 \mathrm{vol} \%$ DI water. In order to investigate the effect of anodization voltage on WCA, two groups of samples were anodized at 20 and $60 \mathrm{~V}$ at room temperature for $4 \mathrm{~h}$ and a third group was left non-anodized for control. Table 3.1 shows the categorization of samples into different groups.

In order to verify effect of anodization voltage on $\mathrm{TiO}_{2}$ nanotubes dimension, a separate group of samples were anodized at 20,40,60,70 and $90 \mathrm{~V}$ for $1 \mathrm{~h}$ in the electrolyte with same composition mentioned above.

In order to verify effect of anodization condition, two groups of roughened samples were anodized at 20 and $60 \mathrm{~V}$ while a third group was not anodized to be used as control. Samples of each group were annealed at 300,450 and $600{ }^{\circ} \mathrm{C}$ for 1.5 and $3 \mathrm{~h}$ to 
verify effect of annealing condition. Finally various tests were performed to characterize the surfaces. \# is used to show number of prepared samples in each group.

Table 3.1 Breakdown of samples into different groups in order to determine the optimal fabrication conditions.

\begin{tabular}{|c|c|c|c|}
\hline Step 1 & Step 2 & Step 3 & Step 4 \\
\hline & & & Non-annealed \#2 \\
\hline & & & Annealed at $300 \mathrm{C}$ for $1.5 \mathrm{~h} \# 2$ \\
\hline & & & Annealed at $300 \mathrm{C}$ for $3 \mathrm{~h} \# 2$ \\
\hline & & Non-anodized \#14 & Annealed at $450 \mathrm{C}$ for $1.5 \mathrm{~h} \mathrm{\# 2}$ \\
\hline & & & Annealed at $450 \mathrm{C}$ for $3 \mathrm{~h} \# 2$ \\
\hline & & & Annealed at $600 \mathrm{C}$ for $1.5 \mathrm{~h} \mathrm{\# 2}$ \\
\hline & & & Annealed at $600 \mathrm{C}$ for $3 \mathrm{~h} \# 2$ \\
\hline & & & Non-annealed \#2 \\
\hline & & & Annealed at $300 \mathrm{C}$ for $1.5 \mathrm{~h} \mathrm{\# 2}$ \\
\hline & & & Annealed at $300 \mathrm{C}$ for $3 \mathrm{~h} \# 2$ \\
\hline Sandblasted \#42 & Acid-etched \#42 & $20 \mathrm{~V}$ anodized $\# 14$ & Annealed at $450 \mathrm{C}$ for $1.5 \mathrm{~h} \# 2$ \\
\hline & & & Annealed at $450 \mathrm{C}$ for $3 \mathrm{~h} \# 2$ \\
\hline & & & Annealed at $600 \mathrm{C}$ for $1.5 \mathrm{~h} \# 2$ \\
\hline & & & Annealed at $600 \mathrm{C}$ for $3 \mathrm{~h} \# 2$ \\
\hline & & & Non-annealed \#2 \\
\hline & & & Annealed at $300 \mathrm{C}$ for $1.5 \mathrm{~h} \# 2$ \\
\hline & & & Annealed at $300 \mathrm{C}$ for $3 \mathrm{~h} \# 2$ \\
\hline & & $60 \mathrm{~V}$ anodized $\# 14$ & Annealed at $450 \mathrm{C}$ for $1.5 \mathrm{~h} \# 2$ \\
\hline & & & Annealed at $450 \mathrm{C}$ for $3 \mathrm{~h} \# 2$ \\
\hline & & & Annealed at $600 \mathrm{C}$ for $1.5 \mathrm{~h} \# 2$ \\
\hline & & & Annealed at $600 \mathrm{C}$ for $3 \mathrm{~h} \# 2$ \\
\hline
\end{tabular}

\section{Annealing Treatment:}

To investigate the effect of annealing temperature and duration on WCA, anodized and non-anodized samples were annealed at 300,450 and $600{ }^{\circ} \mathrm{C}$ for 1.5 and $3 \mathrm{~h}$ durations. A temperature controller was used to set and maintain the furnace temperature. Prior to annealing, samples were cleaned for 30 seconds with DI-water followed by $\mathrm{N}_{2}$ gas drying. The samples were then loaded into the furnace for thermal oxidization at the determined temperatures in ambient air. After heat treatment, samples were slow-cooled by moving them out of the furnace tube slowly at $5 \mathrm{~cm}$ every 7 minutes. This prevents the 
generation of micro-cracks caused by abrupt temperature change on the samples' surface. Table 3.1 shows how samples were categorized into different groups.

Field Emission Scanning Electron Microscopy (FESEM):

Structural characterization of the prepared $\mathrm{TiO}_{2}$ nanotubes was performed using a FESEM (JEOL JSM-6320F). The samples were mounted on an aluminum stub with double-sided conductive carbon tape for imaging.

In order to image dimension of $\mathrm{TiO}_{2}$ nanotubes and determine their aspect ratio, $\mathrm{TiO}_{2}$ nanotubes were removed from substrate by scratching. Detached bundles of nanotubes were placed on a double-sided conductive carbon tape and attached to an aluminum stub for imaging. Finally, ImageJ software was used to measure dimensions of nanotubes.

\section{Energy Dispersive X-Ray (EDX):}

EDX analysis was performed using JEOL JSM-6320F detector. A non-anodized sample and scratched-off nanotubes from surface of an anodized sample placed on carbon tape were verified to evaluate effect of anodization on chemical composition. Noran Voyager EDX software of the instrument was used to determine weight percentage of present elements according to their energy lines.

\section{White Light Interferometry:}

Surface roughness tests were performed on all of the samples after annealing using white light interferometry (NewView 6300, Zygo Corporation, Middlefield, Connecticut, USA). This optical microscope gathers light from the test area of the sample. The objective divides light into 2 paths (one that shines onto the surface of the test material, and another into an internal reference surface). Surface irregularities on the test material cause the measurement wave-front to travel different distances than the reference wave front, which cause interference bands when they are combined. This interference is read by a photo-detector, which translates the interference wave-front into a 3D image. 


\section{Fourier Transform Infrared (FTIR) Spectroscopy:}

Crystalline structure and surface composition of all annealed samples were studied using diffused reflectance FTIR (FTIR, Nicolet, Model \#ADU9700221). Prior to conducting FTIR scans, each sample was left inside the FTIR chamber for $1 \mathrm{~h}$ per purging in order to reduce noise. FTIR spectrum was obtained with $1 \mathrm{~cm}^{-1}$ resolution and 512 scans.

\section{Water Contact Angle measurement:}

To perform WCA measurements sessile drop method was used as standard protocol using a Rame'-Hart NRL CA Goniometer (M\#100-0, S\#2067). A micro-syringe (Hamilton, 802RN) was used to place a $5 \mu \mathrm{L}$ of DI-water droplet onto surfaces. Wettability of the samples were monitored by taking WCA measurements (i) after anodization, (ii) after annealing and (iii) after annealed samples were aged in air.

\section{Statistical Analysis:}

In order to determine the differences in surface hydrophilicity and average surface roughness of various surfaces of Ti-6Al-4V samples, one-way ANOVA test was used. Tukey HSD post hoc analysis was used for pair-wise comparisons within these groups. Statistical software (SPSS v. 20.0, SPSS Inc., Chicago, IL, USA) was used for descriptive and statistical analyses. For all analyses, p-values $<0.05$ was considered statistically significant.

\subsection{Results}

The surfaces have been characterized by various techniques as explained above. The obtained results are as follows. FESEM images (figure 3.1) show surface topography of non-anodized (figure 3.1-A), 20V anodized (figure 3.1-C) and 60V anodized (figure 3.1-E) samples at low magnification. Low-magnification images of anodized surfaces (figures 3.1-C and 3.1-E) show that vanadium-rich beta phase is dissolved. Dissolution of vanadium-rich phase of Ti-V during anodization in acidic electrolyte has previously been reported [40]. Presence of pores formed after dissolution, along with micro-scale 
roughness formed due to sandblasting and acid-etching provide a substrate that can establish long term mechanical bonding after implantation. Figure 3.1 also shows highmagnification images of surface topography for non-anodized (figure 3.1-B), 20V anodized (figure 3.1-D) and 60V anodized surfaces (figure 3.1-F). It is observed that nanotubular structure is formed on anodized surfaces. Diameter of $\mathrm{TiO}_{2}$ nanotubes is about 100 and $50 \mathrm{~nm}$ on 60 and $20 \mathrm{~V}$ anodized sample respectively as marked by red arrows. These dimensions are compatible with previously reported dimensions of $\mathrm{TiO}_{2}$ nanotubes grown on the Ti substrate [138]. 


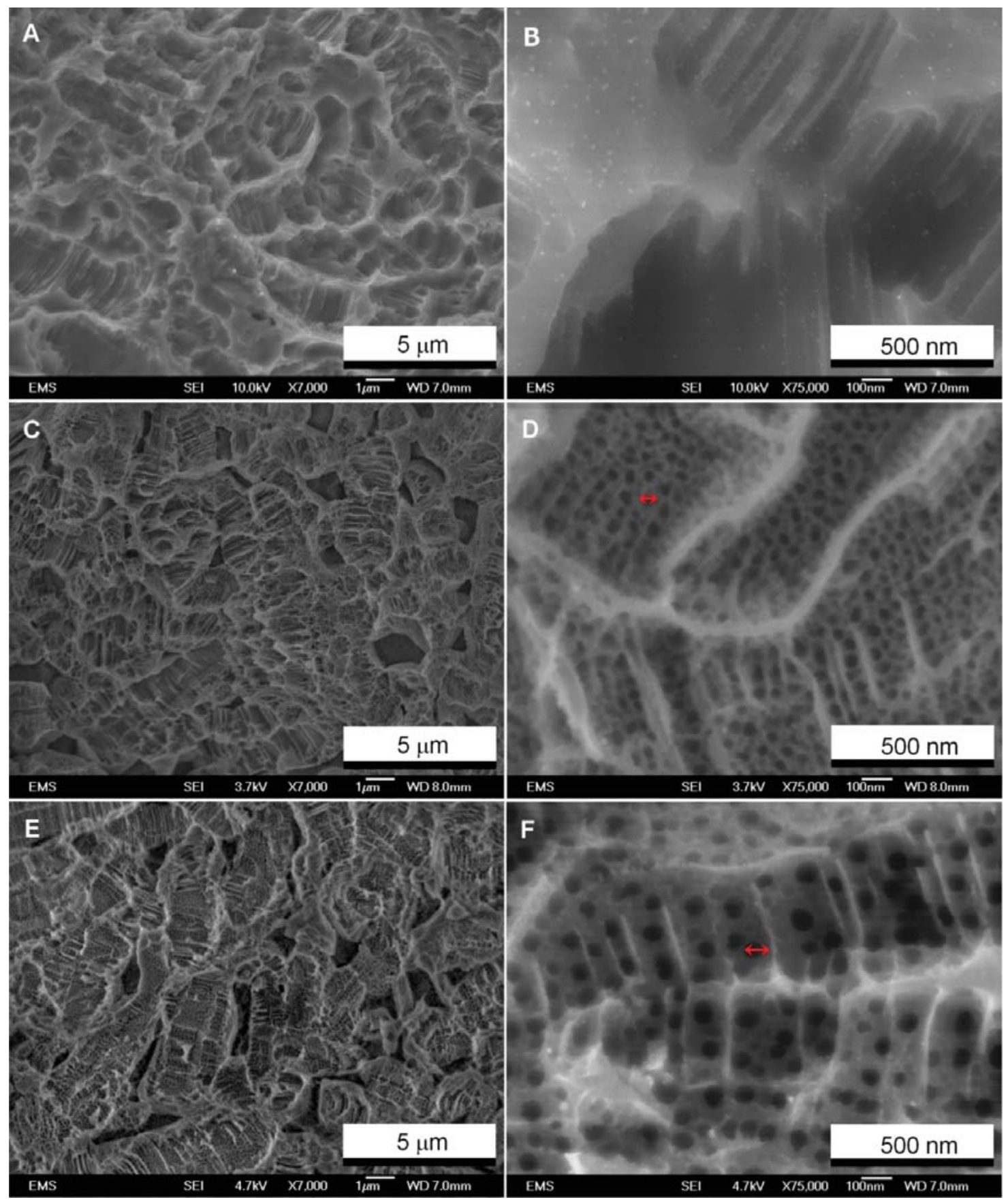

Figure 3.1 FESEM images of the surfaces. Images of non-anodized surface ((A) and (B)), $20 \mathrm{~V}$ anodized surface ((C) and (D)), and $60 \mathrm{~V}$ anodized surface ((E) and (F)) demonstrate formation of $\mathrm{TiO} 2$ nanotubes on anodized samples. Low magnification is shown at left side (A, C and E) and high magnification is shown on the right side $(\mathrm{B}, \mathrm{D}$ and F). 
FESEM images of $\mathrm{TiO}_{2}$ nanotubes anodized at different voltages are shown in (figure $3.2 \mathrm{~A}-\mathrm{D}$ ) for the $20 \mathrm{~V}$ anodized sample (figure $3.2-\mathrm{A}$ ), $40 \mathrm{~V}$ anodized sample (figure 3.2-B), 70V anodized sample (figure 3.2-C) and 90V anodized sample (figure 3.2D). These images demonstrate length and diameter of $\mathrm{TiO}_{2}$ nanotubes increase as the anodization voltage is increased. As expected for nanotubes formed in organic electrolyte, $\mathrm{TiO}_{2}$ nanotubes are closely packed and have smooth walls without any perforation. Round bottom of nanotubes is observed at one side of detached layer while open top of nanotubes is noticed on the other side of nanotubular layer. Length and diameter of nanotubes were measured using ImageJ software and aspect ratio of tubes was calculated. At the bottom of figure 3.2, the diameter of $\mathrm{TiO}_{2}$ nanotubes, length and aspect ratio are plotted versus voltage (figure 3.2 E-G). It can be observed that length and diameter are directly influenced by voltage and aspect ratio also seems to follow the same trend. 

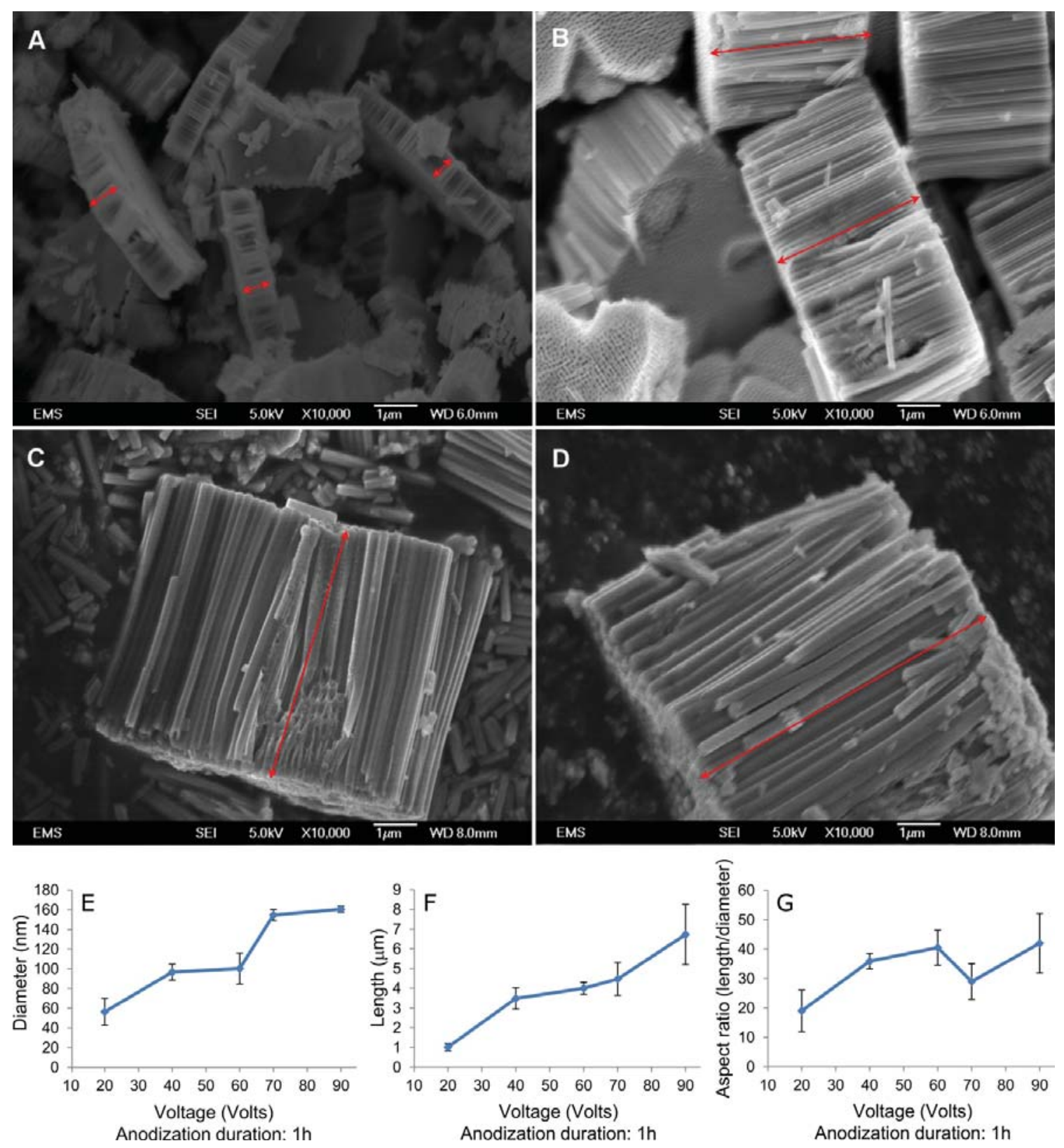

Figure 3.2 FESEM images show effect of anodization voltage on $\mathrm{TiO} 2$ nanotubes dimensions. Diameter and length of $\mathrm{TiO}_{2}$ nanotubes are increased as voltage is increased. (A) Shows 20V anodized sample, (B) 40V anodized sample, (C) 70V anodized sample and (D) $90 \mathrm{~V}$ anodized sample. Effect of anodization voltage on $\mathrm{TiO} 2$ nanotubes dimensions is plotted in figures E-G. (E) Length, (F) diameter and (G) aspect ratio of $\mathrm{TiO} 2$ nanotubes are plotted versus anodization voltage. Length and diameter are 
enhanced as voltage is increased and aspect ratio seems to follow the same trend. Error bars show standard deviation for $\mathrm{n}=3$ samples.

Using the EDX detector, chemical composition analysis was carried out on the surface of a non-anodized sample and scratched-off nanotubes from surface of an anodized sample placed on carbon tape (figure 3.3). A peak indicating the presence of carbon can be seen; the carbon can be either residue of organic electrolyte decomposition or contamination from carbon tape. Comparison of chemical composition of surface before and after anodization suggests that vanadium is dissolved during anodization while oxide is formed (Table 3.2).

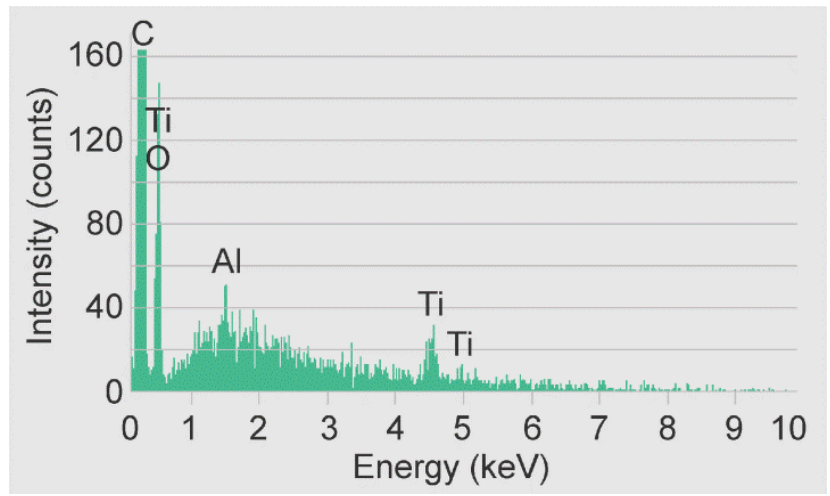

Figure 3.3 Energy Dispersive X-ray analysis. EDX confirms presence of titanium, oxygen and aluminum on the scratched-off nanotubes from surface of an anodized sample placed on carbon tape.

Table 3.2 Comparison of chemical composition of non-anodized surface and nanotubes.

\begin{tabular}{|l|l|l|l|l|l|l|l|}
\hline \multicolumn{1}{|c|}{ Sample } & \multicolumn{6}{|c|}{ Weight\% of elements } \\
\cline { 2 - 10 } & $\mathrm{Ti}$ & $\mathrm{Al}$ & $\mathrm{V}$ & $\mathrm{O}$ & $\mathrm{C}$ & $\mathrm{N}$ & $\mathrm{F}$ \\
\hline Non-anodized & 87.8 & 5.1 & 4.6 & & 1.3 & & \\
\hline Scratched-off nanotubes placed on carbon tape & 32 & 1.6 & 1.5 & 20 & 39.2 & 2.4 & 2.6 \\
\hline
\end{tabular}

Roughness measurements were performed after annealing samples (figure 3.4). Roughness test results are illustrated for non-anodized samples annealed at $450{ }^{\circ} \mathrm{C}$ for $3 \mathrm{~h}$ (figure $3.4-\mathrm{A}$ ), $20 \mathrm{~V}$ anodized sample annealed at $450{ }^{\circ} \mathrm{C}$ for $3 \mathrm{~h}$ (figure $3.4-\mathrm{B}$ ) and $60 \mathrm{~V}$ anodized sample annealed at $450{ }^{\circ} \mathrm{C}$ for $3 \mathrm{~h}$ (figure 3.4-C). Results of the roughness tests show that $60 \mathrm{~V}$ anodized samples have higher average roughness (figure 3.4-D) and 
higher root mean square roughness (data not shown) compared with $20 \mathrm{~V}$ anodized samples. According to Wenzel's equation, enhancement of roughness is correlated with an increase of surface area, which results in enhanced hydrophilicity, protein adsorption, and cell-substrate interaction. 

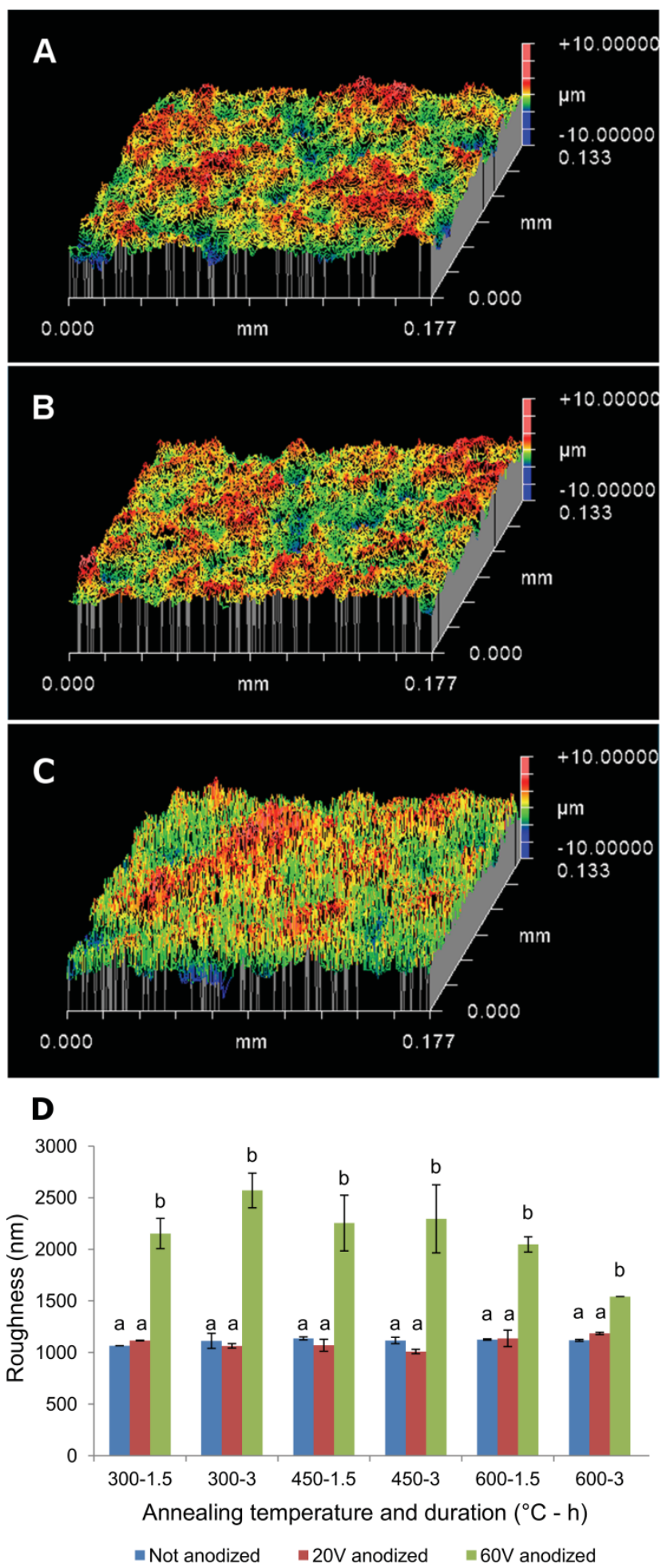

Figure 3.4 White light interferometry test of roughness. Roughness test performed on (A) non-anodized sample, annealed at $450{ }^{\circ} \mathrm{C}$ for $3 \mathrm{~h}$, (B) $20 \mathrm{~V}$ anodized sample, annealed at $450{ }^{\circ} \mathrm{C}$ for $3 \mathrm{~h}$, and (C) $60 \mathrm{~V}$ anodized sample, annealed at $450{ }^{\circ} \mathrm{C}$ for $3 \mathrm{~h} .60 \mathrm{~V}$ anodized 
sample show different roughness compared to $20 \mathrm{~V}$ anodized sample and non-anodized sample. (D) White light interferometry test indicates $60 \mathrm{~V}$ anodized samples possess higher average roughness and higher root mean square roughness compared with $20 \mathrm{~V}$ anodized samples and non-anodized samples. Average roughness is related to surface area and consequently affects surface hydrophilicity. The $\mathrm{x}$-axis indicates the temperature and duration of annealing [Temp $\left({ }^{\circ} \mathrm{C}\right)$ - Time (h)]. a: $p<0.001$ compared to b. Error bars show standard deviation for $\mathrm{n}=2$ samples.

Figure 3.5 shows FTIR spectroscopy results for non-anodized (figure 3.5-A), 20V anodized (figure 3.5-B) and 60V anodized (figure 3.5-C) samples, annealed at different temperatures for $3 \mathrm{~h}$. Obtained spectra confirm the presence of $\mathrm{TiO}_{2}$ along with $\mathrm{CO}_{2}$ and $\mathrm{H}_{2} \mathrm{O}$ from the FTIR system. $\mathrm{CO}_{2}$ peaks are found at $\sim 2367$ and $\sim 667 \mathrm{~cm}^{-1} . \mathrm{H}_{2} \mathrm{O}$ peaks are found at $\sim 3600-3900$ and $\sim 1300-1900 \mathrm{~cm}^{-1}$. As annealing temperature is increased, the intensity of the $\mathrm{TiO}_{2}$ peak is enhanced. In addition, the $\mathrm{TiO}_{2}$ peak shifts from $898 \mathrm{~cm}^{-1}$ for non-annealed samples to $870 \mathrm{~cm}^{-1}$ for annealed samples at 300 and $450{ }^{\circ} \mathrm{C}$. Finally, the $\mathrm{TiO}_{2}$ peak shifts to $830 \mathrm{~cm}^{-1}$ for the samples annealed at $600{ }^{\circ} \mathrm{C}$. This is indicative of the presence of amorphous $\mathrm{TiO}_{2}$ on non-annealed samples, and formation of anatase structure $\mathrm{TiO}_{2}$ after annealing at 300 and $450{ }^{\circ} \mathrm{C}$. Anatase $\mathrm{TiO}_{2},\left(870 \mathrm{~cm}^{-1}\right)$ transforms into rutile $\mathrm{TiO}_{2},\left(830 \mathrm{~cm}^{-1}\right)$ as annealing temperature is increased [139]. This behavior is observed for all samples regardless of anodization condition. For samples annealed at 300 and 450 ${ }^{\circ} \mathrm{C}, \mathrm{TiO}_{2}$ with crystalline anatase structure is formed as well as rutile structure. For $60 \mathrm{~V}$ anodized samples (figure 3.5-C), extra features are observed in the region $950-1350 \mathrm{~cm}^{-1}$ which represent Al-OH, Al-O-Al, $\mathrm{V}_{2} \mathrm{O}_{5}$ and $\mathrm{Al}_{2} \mathrm{O}_{3}$ [140-146]. 

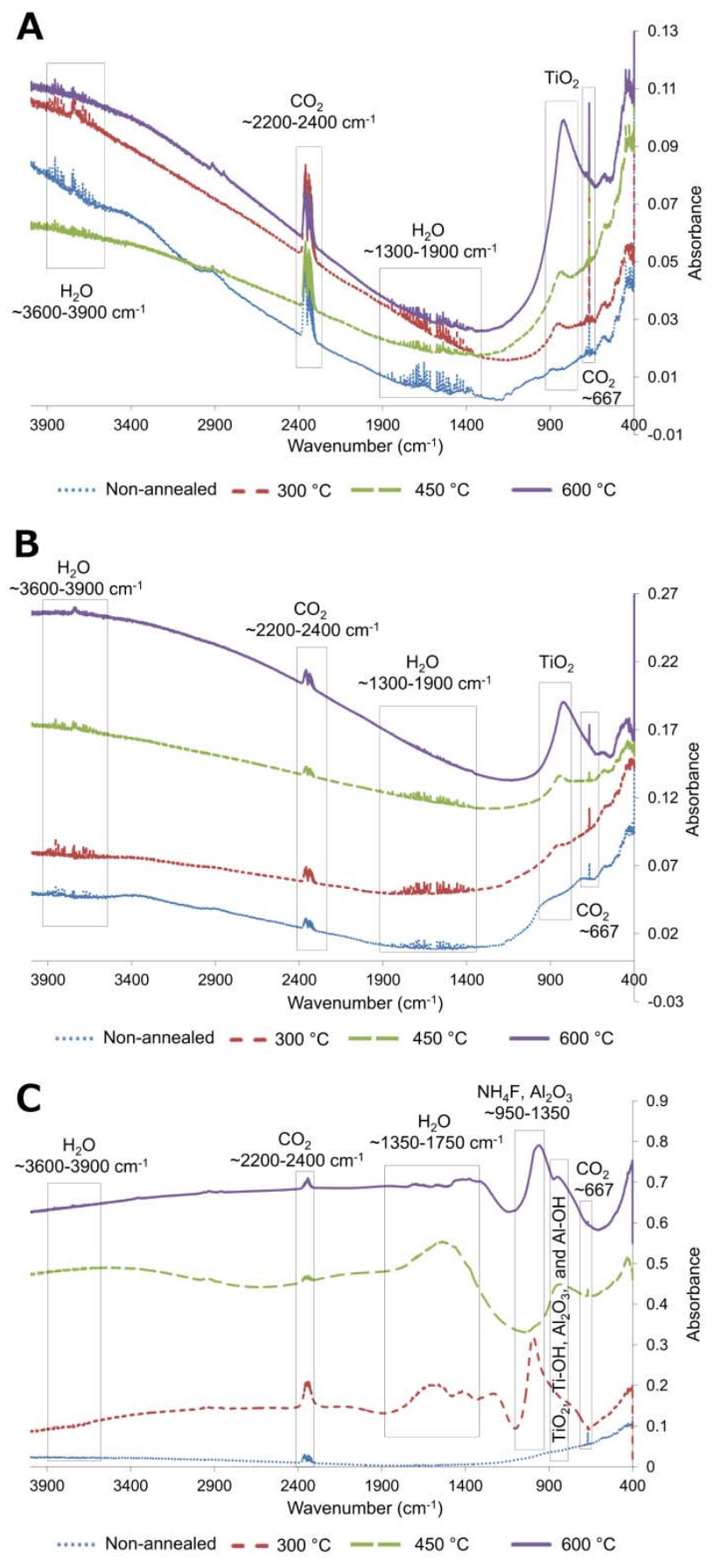

Figure 3.5 FTIR spectroscopy. FTIR spectra are shown for (A) non-anodized samples, (B) $20 \mathrm{~V}$ anodized samples and (C) $60 \mathrm{~V}$ anodized samples annealed at different temperatures for $3 \mathrm{~h}$. The obtained spectra confirm the presence of $\mathrm{TiO}_{2}$ along with $\mathrm{CO}_{2}$ and $\mathrm{H}_{2} \mathrm{O}$ from the FTIR system. As annealing temperature is increased, the intensity of 
the $\mathrm{TiO}_{2}$ peak is enhanced. In addition, the $\mathrm{TiO} 2$ peak shifts from $898 \mathrm{~cm}^{-1}$ for nonannealed samples with amorphous structure to $870 \mathrm{~cm}^{-1}$ for annealed samples at 300 and $450{ }^{\circ} \mathrm{C}$. Finally, the $\mathrm{TiO}_{2}$ peak shifts to $830 \mathrm{~cm}^{-1}$ for the samples annealed at $600{ }^{\circ} \mathrm{C}$.

Using WCA measurements, surface wettability behavior was investigated for anodized samples at various voltages along with different annealing temperatures and durations (figure 3.6 A-C). WCA of non-anodized (figure 3.6-A) and 20V anodized samples (figure 3.6-B) were high, ranging from 50 to $120{ }^{\circ} \mathrm{C}$. However, $60 \mathrm{~V}$ anodized samples show WCA below $5{ }^{\circ} \mathrm{C}$ (figure 3.6-C). After annealing, all groups demonstrated WCA below $5{ }^{\circ} \mathrm{C}$. WCA measurements of the samples after being aged in air for 11 days, show increase in WCA for non-anodized and 20V anodized samples, ranging from 50 to $120^{\circ} \mathrm{C}$. However, $60 \mathrm{~V}$ anodized samples, maintained their low WCA after aging up to 11 days, regardless of annealing condition. 


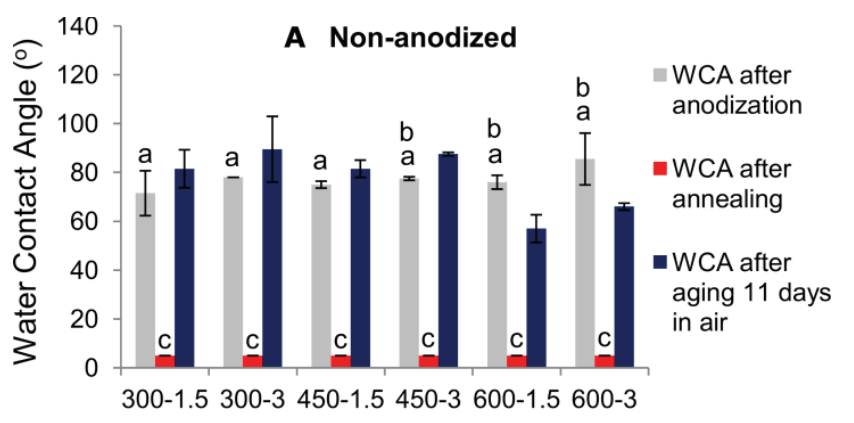

Annealing temperature and duration $\left({ }^{\circ} \mathrm{C}-\mathrm{h}\right)$

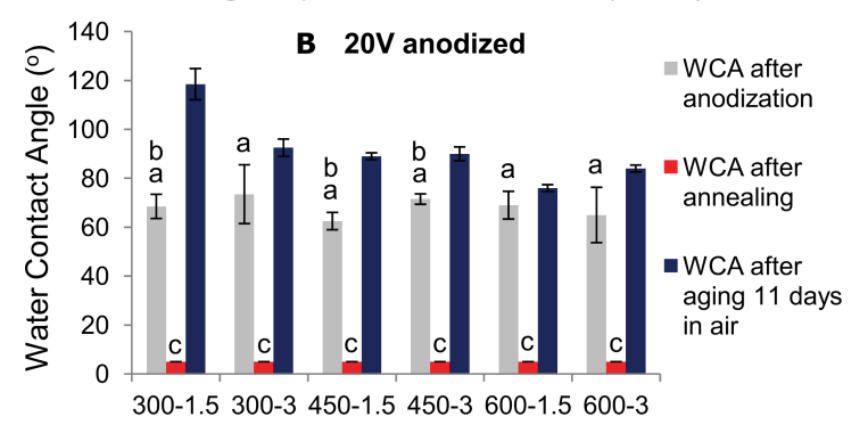

Annealing temperature and duration $\left({ }^{\circ} \mathrm{C}-\mathrm{h}\right)$

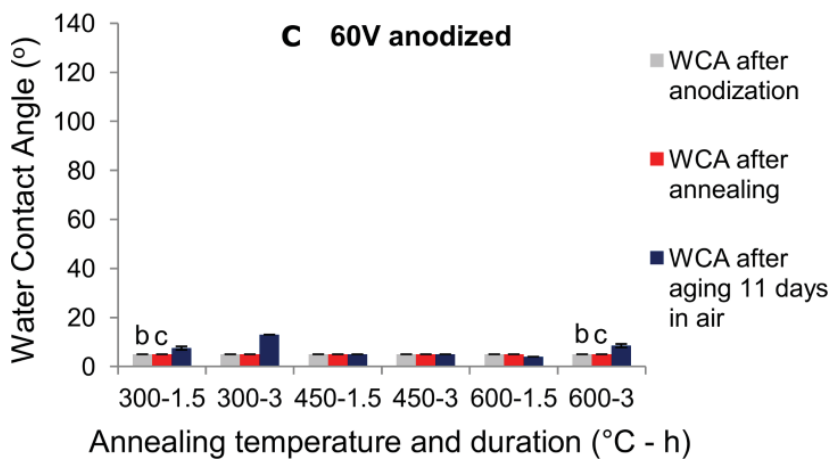

Figure 3.6 WCA measurements of the samples fabricated at different anodization and annealing conditions. WCA are shown for (A) not anodized samples, (B) 20V anodized samples and (C) $60 \mathrm{~V}$ anodized samples. $60 \mathrm{~V}$ anodized samples possess low WCA after anodization and they are able to maintain their hydrophilicity when aged in air up to 11 days. The $\mathrm{x}$-axis indicates the temperature and duration of annealing $\left[\right.$ Temp $\left({ }^{\circ} \mathrm{C}\right)$ - Time (h)]. a: $p<0.05$ compared to annealed group, b: $p<0.05$ compared to aged group, c: $p<0.05$ compared to aged group. Error bars show standard deviation for $\mathrm{n}=2$ samples. 
In order to verify effect of annealing temperature on aging behavior of $\mathrm{TiO}_{2}$ nanotubes, WCA measurements were performed up to 60 days. WCA measurements after prolonged aging indicate that annealing condition affects the ability of surface to maintain its hydrophilicity (figure 3.7). As expected, $60 \mathrm{~V}$ anodized samples, annealed at $300{ }^{\circ} \mathrm{C}$ for $3 \mathrm{~h}$, are more able to maintain surface hydrophilicity compared with nonannealed samples. Interestingly, the $60 \mathrm{~V}$ anodized samples annealed at $600{ }^{\circ} \mathrm{C}$ for $3 \mathrm{~h}$ provide the highest ability in maintaining surface hydrophilicty. The samples that were prepared under optimum conditions (60V anodized samples annealed at $600{ }^{\circ} \mathrm{C}$ for $3 \mathrm{~h}$ ) provide stable, non-aging, hydrophilic surfaces, which maintain hydrophilicity for at least up to 60 days.

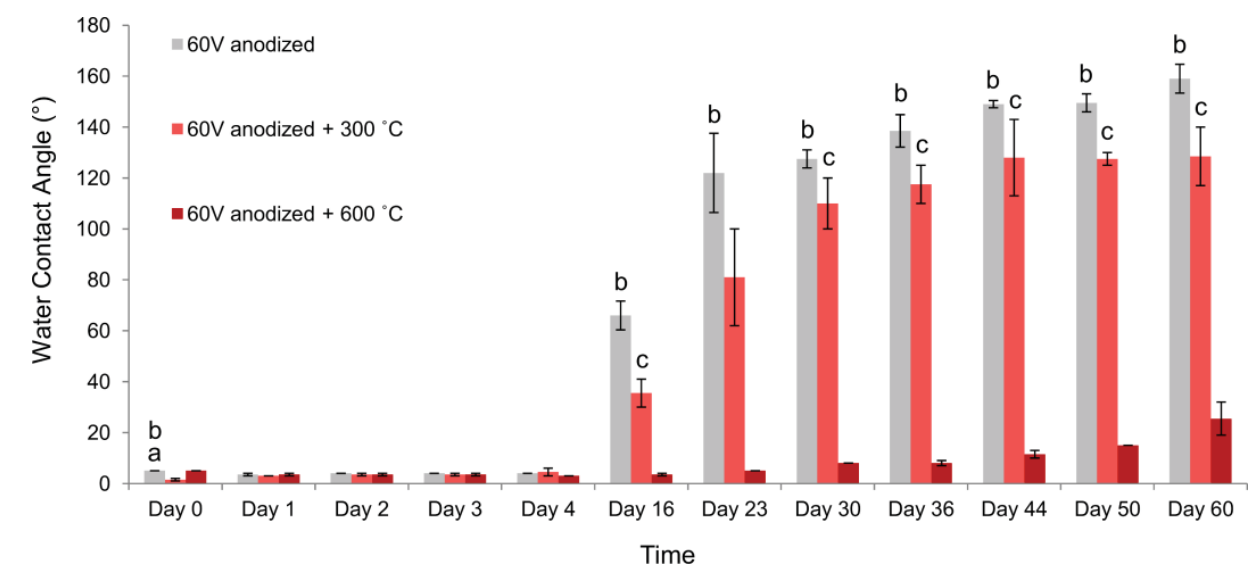

Figure 3.7 Effect of annealing temperature on aging behavior of $60 \mathrm{~V}$ anodized samples. Gray bars are non-anodized samples, light red bars are anodized samples that are then annealed at $300{ }^{\circ} \mathrm{C}$ for $3 \mathrm{~h}\left(60 \mathrm{~V}+300^{\circ} \mathrm{C}\right)$ and dark red bars are anodized samples that are then annealed at $600{ }^{\circ} \mathrm{C}$ for $3 \mathrm{~h}\left(60 \mathrm{~V}+600{ }^{\circ} \mathrm{C}\right) .60 \mathrm{~V}$ anodized samples that are then annealed at $600{ }^{\circ} \mathrm{C}$ for $3 \mathrm{~h}$, are significantly more able to maintain their hydrophilicity. a: $p<0.05$ compared to $60 \mathrm{~V}+300{ }^{\circ} \mathrm{C}$ group, b: $p<0.05$ compared to $60 \mathrm{~V}+600{ }^{\circ} \mathrm{C}$ group, c: $p<0.05$ compared to $60 \mathrm{~V}+600{ }^{\circ} \mathrm{C}$ group. Error bars show standard deviation for $\mathrm{n}=2$ samples.

\subsection{Discussion}

EDX results confirm the presence of titanium, vanadium and aluminum as components of Ti-V on non-anodized surface. However, scratched-off nanotubes show 
presence of oxygen; and relatively lower weight percentage of vanadium possibly due to the dissolution of vanadium during anodization (Table 3.2). The fluorine is hypothesized to be either residual or part of the chemical structure on the surface. The electrolyte is ammonium fluoride in ethylene glycol and water, and the fluorine is part of the chemical process of nanotube formation. The nitrogen may also be from the electrolyte. It should be noted that $\mathrm{Ti}$ is $44 \mathrm{~g} / \mathrm{mol}$ and $\mathrm{O}_{2}$ is $32 \mathrm{~g} / \mathrm{mol}$, or oxygen weight $\%$ is $72 \%$ of the weight $\%$ of $\mathrm{Ti}$. Therefore for the $\mathrm{TiO}_{2}$ nanotube values, the 20 weight $\%$ given for $\mathrm{O}$ is about $63 \%$ of the weight $\%$ for $\mathrm{Ti}$, which indicates presence of oxygen equivalent to $\mathrm{TiO}_{1.7}$. In literature, the nanotube surface is a mixture of $\mathrm{TiO}_{2}$ and $\mathrm{TiOH}$, which may explain the lower oxygen count. The Noran Voyager EDX software of the FESEM instrument was used to determine weight percentage of the elements according to their energy lines. The software fits a $\mathrm{K} \alpha+\mathrm{K} \beta$ peak shape to the data to arrive at the $\mathrm{K}$ intensities. While Ti $\mathrm{K} \beta_{1}$ peak (4.93 keV) overlaps with $\mathrm{V} \mathrm{K} \alpha_{1,2}(4.93 \mathrm{keV})$, the $\mathrm{V} \mathrm{K} \beta_{1}$ peak $(5.42 \mathrm{keV})$ has no overlap so it is possible to conclude $\mathrm{V}$ is decreased during anodization. Considering that the ratios of $\mathrm{K} \alpha$ to $\mathrm{K} \beta$ are very well known for transition metal $\mathrm{K}$ shells, the software is capable of deconvoluting Ti $\mathrm{K} \beta$ from $\mathrm{V} \mathrm{K} \alpha$. However, while the precision of oxygen quantification is questionable since Ti $\mathrm{L} \alpha(0.45 \mathrm{keV})$ and V $\mathrm{L} \alpha(0.51 \mathrm{keV})$ overlap the $\mathrm{O} \mathrm{K} \alpha(0.52 \mathrm{keV})$, we are able to observe a relative increase in oxygen between the non-anodized and anodized samples.

Our results indicate that hydrophilicity after anodization is highly affected by applied anodization voltage. Before annealing, 60V anodized samples show higher hydrophilicity compared to control (non-anodized) and $20 \mathrm{~V}$ anodized samples (figure 3.6). Previous studies also show that surfaces of both $\mathrm{Ti}$ and $\mathrm{Ti}-\mathrm{V}$ become hydrophilic after anodization and hydrophilicity is further increased as anodized surface in annealed $[137,147]$. This behavior can be attributed to the increase in surface area after anodization, which results in enhancement of hydrophilicity, available area for adsorption, interaction, and consequently cell adhesion $[9,34,48,49]$. Enhancement of roughness can generally lead to either the increase or decrease of water contact angle. In the case of formation of nanotubes on the surface of titanium dioxide, it has been widely reported that formation of nanotubes results in reduction of water contact angle [65,137], due to presence of $\mathrm{OH}^{-}$groups on the anodized surface in the form of $\mathrm{Ti}(\mathrm{OH})_{4}$ [137]. 
Therefore, it is assumed that air is not trapped underneath the liquid and Wenzel's equation can be applied for the $\mathrm{TiO}_{2}$ nanotubular surface. Based on the Wenzel relation, as surface roughness of a hydrophilic surface is enhanced, surface hydrophilicty is increased [148]. Our results, in compliance with previous studies [34,65], show that surface roughness is increased after anodization (figures 3.4). As anodization voltage increased, the surface area and nanotube size was also increased proportionally (figure 3.1 E-G). Thus, $60 \mathrm{~V}$ anodized samples show higher hydrophilicity compared to that of control (non-anodized) and $20 \mathrm{~V}$ anodized samples. In addition, application of higher voltage during anodization of both $\mathrm{Ti}$ and $\mathrm{Ti}-\mathrm{V}$ materials can result in formation of asanodizaed $\mathrm{TiO}_{2}$ nanotubes with some amount of crystalline structure [65] which, in turn, plays an important role in surface hydrophilicity. Higher anodization voltage also promotes formation of anatase crystalline structure obtained after heat treatment [147].

Our results indicate that annealing significantly decreases WCA (figure 3.6). Nanotubular surfaces $\left(\mathrm{WCA}>50^{\circ}\right.$ ) are transformed to superhydrophilic surfaces (WCA < $5^{\circ}$ ) after annealing, regardless of annealing and anodization conditions. It seems surface structure - in terms of crystallinity and morphology - is the main influence on hydrophilicity, while electrolyte components present on the surface do not crucially affect WCA. When $\mathrm{NH}_{4} \mathrm{~F}$ containing electrolyte is used for anodization, fluorine is present on the anodized surfaces. The fluorinated compound formed during anodization is water soluble and potentially affects hydrophilicity. Atsuta et al. reported hydrophilicity is improved after treatment with $\mathrm{NH}_{4} \mathrm{~F}$ [149]; however, fluorine is thermally decomposed during annealing $[9,56,64,150]$ and can not be responsible for hydrophilicity of annealed surfaces. Crystallinity and morphology are the factors that control surface hydrophilicity.

WCA measurements after aging (figure 3.6) demonstrate that surface hydrophilicity of non-anodized and $20 \mathrm{~V}$ anodized samples decreases after 11 days of aging in air; however, annealed $60 \mathrm{~V}$ anodized samples maintain their hydrophilicity up to 11 days aging in air, regardless of annealing condition. The effect of annealing temperature demonstrated that the nanotubes that are obtained by $60 \mathrm{~V}$ anodization and annealing at $300{ }^{\circ} \mathrm{C}$ lose their hydrophilicity after 16 days of aging (figure 3.7). Nevertheless, nanotubes that are obtained by $60 \mathrm{~V}$ anodization and annealing at $600{ }^{\circ} \mathrm{C}$ maintain their hydrophilicity even after 60 days of aging. 
It is known that hydrophilicity is related to the presence of $\mathrm{OH}^{-}$groups on the surface in the form of $\mathrm{Ti}(\mathrm{OH})_{4}$ after anodization. When anodized surface is aged in air, hydroxyl groups are transmitted to air to reach surface hydroxylation/dehydroxylation equilibrium. Since $\mathrm{TiO}_{2}$ is more stable than the $\mathrm{Ti}(\mathrm{OH})_{4}$, following reaction occurs: $\mathrm{Ti}(\mathrm{OH})_{4} \rightarrow \mathrm{TiO}_{2}+2 \mathrm{H}_{2} \mathrm{O}$ [137]. Consequently, aged surfaces lose their hydrophilicity. Our data suggests that a relation exists between surface hydroxylation/dehydroxylation equilibrium and surface crystalline structure. Anatase crystalline structure is more potent in preventing the establishment of hydroxylation/dehydroxylation equilibrium compared with amorphous $\mathrm{TiO}_{2}$, while rutile crystalline structure provides the greatest resistance to the hydroxylation/dehydroxylation equilibrium process (figure 3.5).

Dissolution of $\beta$ phase of Ti-V along with micro-scale roughness of surface formed by sand-blasting would in turn assist mechanical bonding at bone implant interface. Optimized nano scale structure of nanotubes provides promising surfaces for implants used in dentistry and orthopedic applications.

\subsection{Conclusions}

Hydrophilic nanotubular surfaces obtained by anodization improve cellular interaction. Considering the key role of surface hydrophilicity, we have studied optimization of anodization and annealing conditions. The following conclusions can be made from this study:

$>$ Our results indicate that the hydrophilicity of $\mathrm{TiO}_{2}$ nanotubes before annealing is highly affected by the anodization voltage. Before annealing, $60 \mathrm{~V}$ anodized samples show low WCA $\left(<5^{\circ}\right)$, while non-anodized and 20V anodized samples show high WCA $\left(>50^{\circ}\right)$. Lower WCA of $60 \mathrm{~V}$ anodized samples can be due to presence of nanotubes with larger dimensions and higher surface roughness. However, the main factor that affects the maintenance and stability of the obtained hydrophilic $\mathrm{TiO}_{2}$ nanotubular surfaces is the annealing temperature.

$>$ Annealing significantly decreases the WCA $\left(<5^{\circ}\right)$. Nanotubular surfaces are transformed to hydrophilic surfaces after annealing, regardless of annealing and anodization conditions. However, WCA measurements after aging demonstrate that 
surface hydrophilicity of non-anodized and 20V anodized samples decreases within 11 days of aging, while $60 \mathrm{~V}$ anodized samples maintain their hydrophilicity longer than 11 days. Anodization at high voltages partially promotes formation of crystalline structure which in turn enhances surface hydrophilicity.

$>$ The effect of annealing temperature demonstrated that nanotubular surfaces that are obtained by $60 \mathrm{~V}$ anodization and annealing at $300{ }^{\circ} \mathrm{C}$ lose their hydrophilicity after 16 days of aging. Nevertheless, nanotubeular surfaces that are obtained by $60 \mathrm{~V}$ anodization and annealing at $600{ }^{\circ} \mathrm{C}$ maintain their hydrophilicity up to 60 days. This behavior suggest that hydroxylation/dehydroxylation equilibrium on surface is established slower as amorphous structure is transformed to anatase crystalline structure and the slowest equilibrium process occurs when anatase is transformed to rutile.

Therefore, we conclude that in order to obtain nanotubes that do not age for several weeks in ambient conditions, an annealing temperature of $450{ }^{\circ} \mathrm{C}$ above is required.

\section{Acknowledgements}

The authors would like to thank Prof. Craig Friedrich, MTU, Multi-Scale Technologies Institute for all of his contributions. 


\title{
Chapter 4
}

\section{Titanium Dioxide Nanotubes for Prolonged Release of Drug Molecules*}

\author{
Azhang Hamlekhan ${ }^{1}$, Suman Sinha-Ray ${ }^{2,9}$, Christos Takoudis $^{3}$, Mathew T. Mathew ${ }^{4}$, \\ Cortino Sukotjo ${ }^{6}$, Alexander L. Yarin ${ }^{2,7,10}$, Tolou Shokuhfar ${ }^{1,2,5,8,10}$ \\ ${ }^{1}$ Department of Mechanical Engineering, Multi-Scale Technologies Institute, \\ Michigan Technological University \\ ${ }^{2}$ Department of Mechanical and Industrial Engineering, University of Illinois at Chicago \\ ${ }^{3}$ Department of Bioengineering, University of Illinois at Chicago \\ ${ }^{4}$ Department of Orthopedics, Rush University Medical Center, Chicago \\ ${ }^{6}$ Department of Restorative Dentistry, College of Dentistry, University of Illinois at \\ Chicago \\ ${ }^{7}$ College of Engineering, Korea University, Seoul, S. Korea \\ ${ }^{8}$ Department of Physics, University of Illinois at Chicago \\ ${ }^{9}$ Corporate Innovation Center, United States Gypsum, Libertyville, IL \\ ${ }^{10}$ Corresponding authors: ayarin@uic.edu,tshokuhf@mtu.edu
}

\begin{abstract}
Formation of titanium dioxide nanotubes (TNTs) on titanium surface holds great potential for promoting desirable cellular response. However, prolongation of drug release from these nano-reservoirs remains to be a challenge. In our previous work TNTs were successfully loaded with drug. In this study the effect of TNTs dimensions on

*The material contained in this chapter has been submitted to the journal of Advanced Healthcare Materials. As the first author of this publication, I have done the fabrication of nanotubes, FESEM, drug loading, drug release measurements, literature review and writing the manuscript.
\end{abstract}


prolongation of drug release was quantified aiming the introduction of a simple novel technique which overcomes complications of previously introduced methods. Different groups of TNTs with different lengths and diameters were fabricated. Samples were loaded with a model drug and rate of drug release over time was monitored. The relation of the drug release rate to the TNT dimensions (diameter, length, aspect ratio and volume) was established. The results show that an increase in any of these parameters increases the duration of the release process. However, the strongest parameter effecting the drug release is the aspect ratio. It was found that TNTs with higher aspect ratios release drug slower.

Keywords: $\mathrm{TiO}_{2}$ nanotubes, Anodization, Drug release, Drug delivery, Intercalation

\subsection{Introduction}

Titanium and its alloys have recently attracted attention for several advantages they provide as dental/orthopedic implants. [6] They possess great biocompatibility in terms of low ion release,[7] excellent corrosion resistance,[120] great mechanical properties in terms of high hardness, low elastic modulus and low density.[9$11,13,121,122]$ Biocompatibility of titanium is related to the formation of native oxide layer on its surface once it is exposed to air. This layer of native oxide $\left(\mathrm{TiO}_{2}\right)$ with thickness of $2-5 \mathrm{~nm}$ protects the bulk material from corrosion [15] and makes it bioinert.[16] Despite these beneficial properties, in some cases titanium implants are encapsulated in vivo by fibrous tissue and show lack of osteointegrity which can lead to infection and implant failure.[123] Moreover, in case of dental implants, dentists are unable to test the sterility of a tooth's apex. Therefore, an asymptomatic endodonticallytreated tooth may be harboring a chronic infection, which may lead to implant failure.[151]

To improve implant viability, nanotechnology holds certain advantages through the modification of implant surfaces.[51,152] Lately, employing anodization technique for formation of TNTs on the surface of titanium implants has attracted much attention.[19-21] The anodized nano-tubular surface has shown controlled-order and 
promising potential for biomedical application, due to increased osteoblast cell adhesion and function,[19-21] increased growth of hydroxyapatite,[27,28] and influence on cellular behavior and tissue integration.[29] Physical cues of the environment affect cellular function.[153] Desirable cellular response of different cell lines is increased on nano-tubular surface compared to flat machined surface. Such enhancement is due to increase in surface area which results in enhancement of available area for cell-substrate interaction, increase in surface energy, hydrophilicity, protein adsorption and, consequently, cell adhesion.[9,33,34,48-50] Formation of TNTs on Ti substrate has shown to decrease water contact angle (WCA).[65] Reduction of WCA is highly desirable since low WCA is correlated with enhanced protein adsorption on the surface and, consequently, improves bone-cell interaction after implantation.[53] In addition, hydrophilic (low WCA) TNTs, can intercalate drug molecules very effectively.[154] The drug- loaded TNTs have shown enhanced osteoblast functionality and consequently can result in improved osseointegration.[119]

Localized drug delivery has been developed to overcome systemic side effects and delivery deficiencies. Many drugs are not effectively delivered via systemic routes. For example when antibiotics such as Neomycin and Gentamicin [105] are taken orally, they are absorbed from the small intestine and inactivated. Bone Morphogenic Protein 2 (BMP-2), as an osteogenic factor, is often delivered intravenously or topically. However, avascular tissue forms after surgery inhibits delivery of drug to implant-tissue interface. Increasing systemic doses to overcome this disadvantage leads to organ toxicity.[87] In order to deliver drug locally, TNTs have shown potential to be loaded with drug and antibacterial agents. Osteoblast response is improved when nanotubes are loaded with antibiotics.

Although localized drug delivery provides targeted release of drug and a certain control of drug, release over time remains a challenge. Preventing sudden release of drug after implantation avoids denaturation of drug and enhances its efficiency.[115] Formation of nano-tubular structure on machined surface increases the amount of loaded drug and slightly prolongs its release.[116] Several methods were explored for further prolonging drug release form TNTs. Hu et al. coated the surface of drug-loaded TNTs with multiple layers of gelatin and chitosan to further retard drug release.[115] Although 
controlled release of drug from TNTs was successfully achieved through this technique, the polymeric coating that was used in this method prevented direct cell attachment and cellular interaction with the nanotubes. On the other hand, when TNTs are loaded with polymer micelles as drug carriers,[117] cellular response can be induced. Degradation of the polymer may induce inflammation; and therefore in it is preferable not to use polymeric-based drug intercalation. One suitable, simple, and cost-effective method for prolonging drug release would be, increasing TNTs length. In fact, it has been reported that release of antibiotic drug molecules is longer from elongated TNTs in comparison to shorter TNTs.[91] In another report, nanotubes formed from $\mathrm{Ti}-4 \mathrm{Zr}-22 \mathrm{Nb}-2 \mathrm{Sn}$ also showed prolonged drug release from longer nanotubes.[118] It is sensible to hypothesize that more drug molecules would be intercalated within TNTs with larger diameter and length. However, there is a lack of research on the effect of TNTs diameter and length on the release rate of intercalated drug molecules. TNT dimensions can be controlled by applying different conditions including electrolyte type and composition,[86] electrolyte $\mathrm{pH},[85]$ anodization voltage magnitude and anodization duration. Diameter and length of TNTs is increased as applied voltage and anodization duration are increased.[34,81-83] In the present study we explore the effect of TNTs aspect ratio on the drug release rate and duration.

\subsection{Results}

FESEM images (Figure 4.1) show that the nano-tubular structure is formed on all anodized surfaces. As expected for nanotubes formed in organic electrolyte, TNTs are closely packed together and have smooth walls without any perforations. Round bottom of nanotubes is visible at one side of detached layer, while open top of nanotubes is noticed on the other side of the nano-tubular layer. Length and diameter of TNTs are increased as the anodization voltage is increased. 


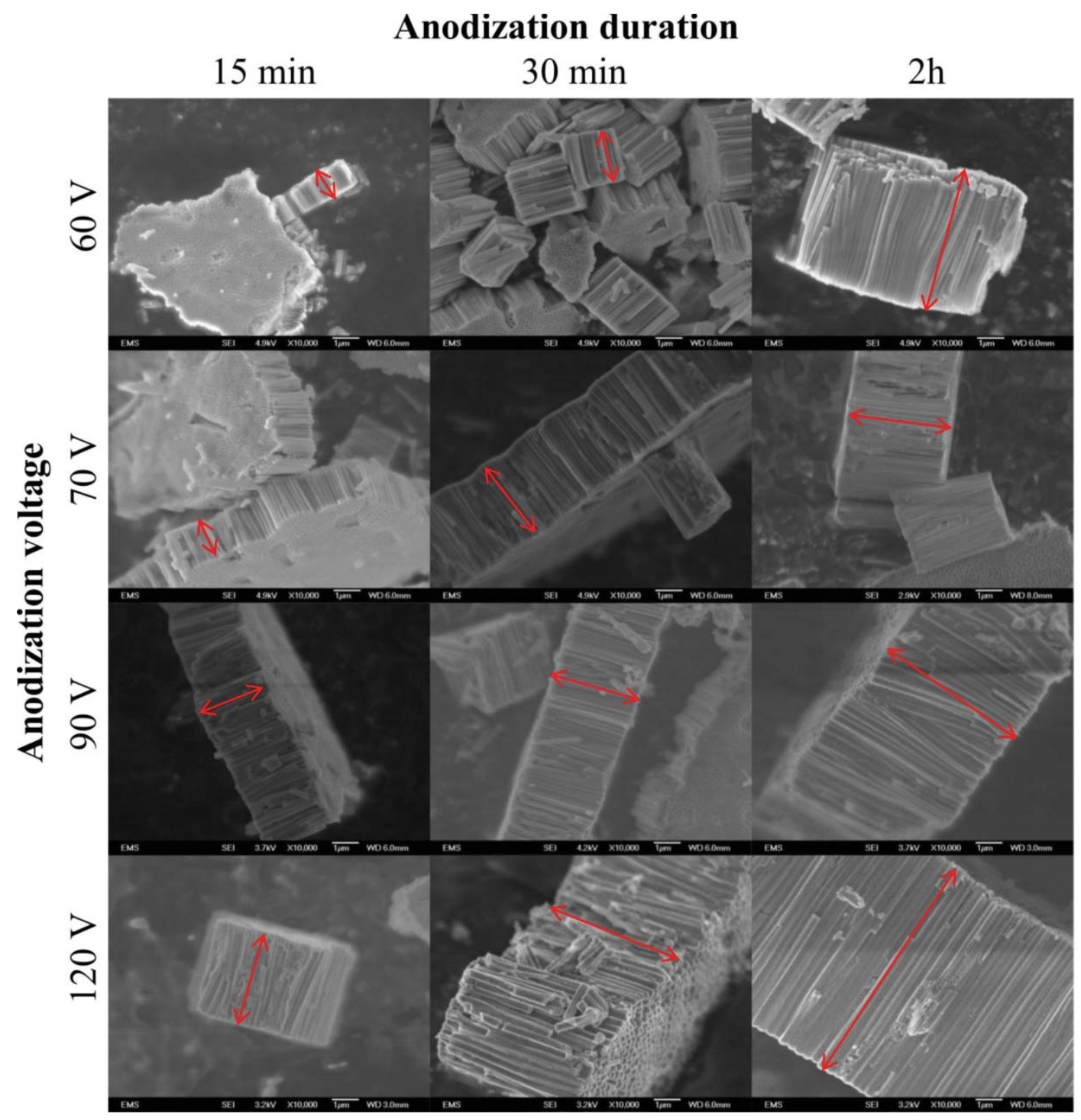

Figure 4.1 FESEM images of nanotubes formed after $15 \mathrm{~min}, 30 \mathrm{~min}$ and $2 \mathrm{~h}$ anodization at different voltages of $60 \mathrm{~V}, 70 \mathrm{~V}, 90 \mathrm{~V}$ and $120 \mathrm{~V}$. Length of nanotubes is increased as anodization duration is increased. Diameter and length of nanotubes is increased as anodization voltage is increased.

TEM image of TNT arrays before drug loading and a single drug-loaded TNT is shown in Figure 4.2-A and 4.2-B respectively. Figure 4.2-C shows the elemental composition of the $\mathrm{TiO}_{2}$ nanotube as prepared. 

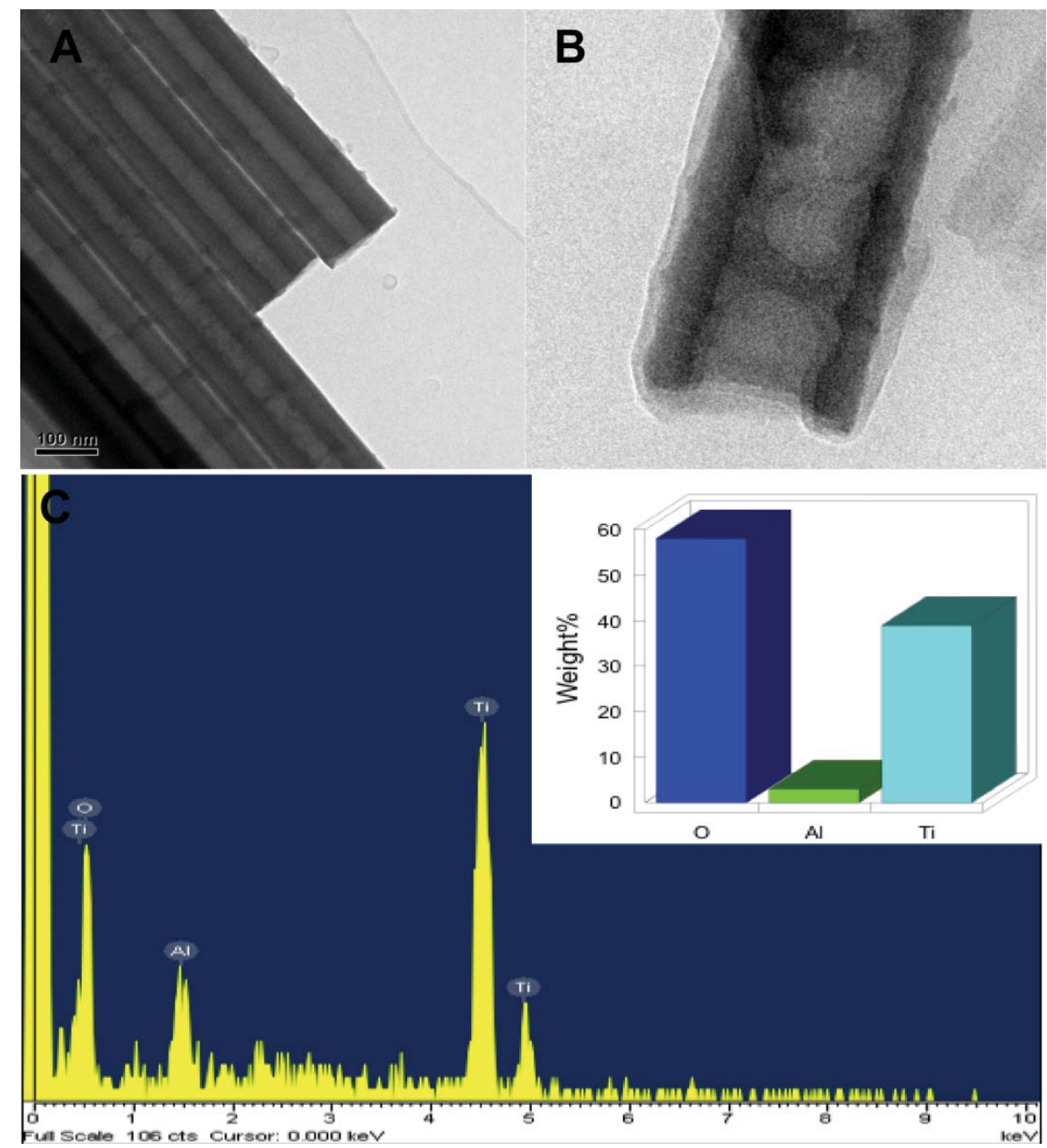

Figure 4.2 TEM image of TNT arrays before drug loading (A) and a single drug-loaded TNT is shown (B). Scale bar is $100 \mathrm{~nm}$. The EDS results (C) shows the presence of 40 wt $\%$ titanium, $60 \mathrm{wt} \%$ oxygen, and less than $5 \mathrm{wt} \%$ aluminum elements in a single TNT.

Using FESEM images, length and diameter of nanotubes versus voltage were measured using ImageJ software and, accordingly, the aspect ratio and the external volume of TNTs versus voltage were calculated. They are presented in Figure 4.3. The results show that length and diameter of TNTs increase as either the anodization voltage or anodization duration is increased (cf. Figure 4.3-A-B). However, the aspect ratio does not seem to follow the same trend (cf. Figure 4.3-C). External volume of TNTs increases as the anodization voltage or duration is increased (Figure 4.3-D). 

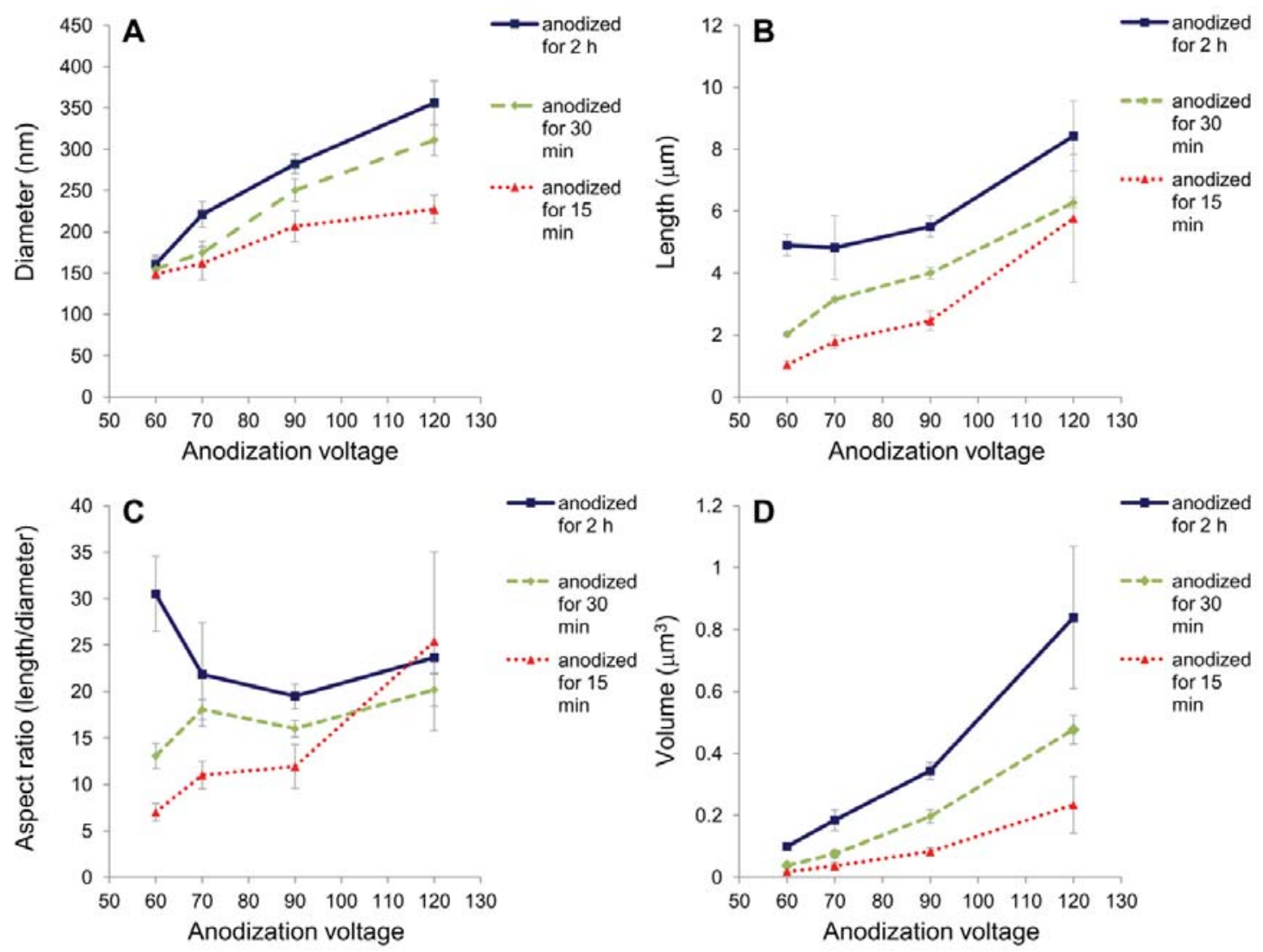

Figure 4.3 Effect of anodization voltage on (A) diameter, (B) length, (C) aspect ratio, and (D) volume of TNTs.

Figure 4.4 shows the release profile of all TNT groups studied. Comparing the profiles with the TNT dimensions specified in Figure 4.3, one can conclude that larger nanotubes with larger volume release larger amount of drug. In other words, larger nanotubes are loaded with higher amount of drug. All types on TNTs show a higher amount of drug released in comparison with the controls. Two control groups were used in this study: (i) anodized at $60 \mathrm{~V}$ for $1 \mathrm{~h}$ - not loaded with drug, and (ii) non-anodized (polished) - loaded with drug. 


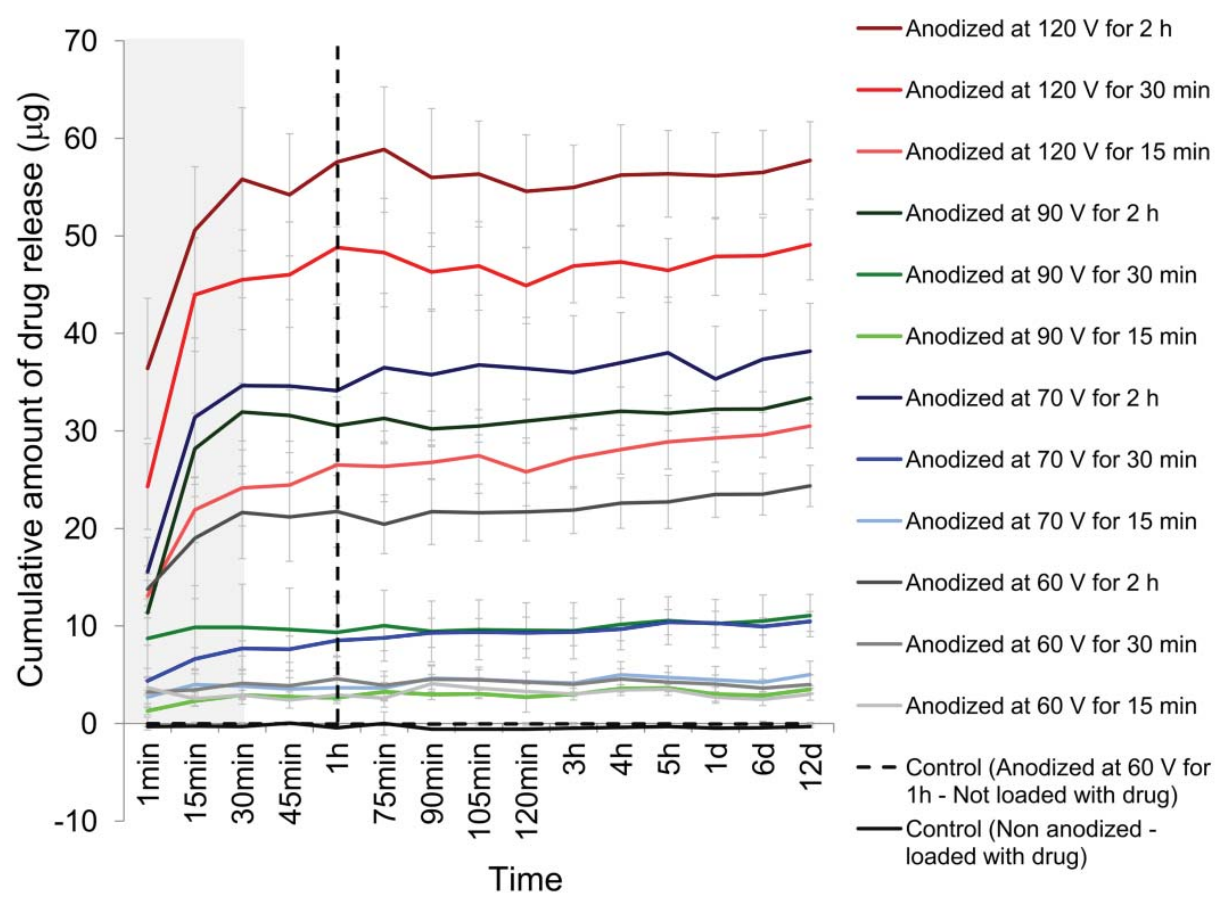

Figure 4.4 Release profiles of TNT groups. Shaded domain corresponds to active release stage which lasts for 30 minutes. During this stage, most of the loaded drug is released from nanotubes into aqueous environment. Some groups of TNTs release the overall amount of loaded drug in less than $15 \mathrm{~min}$, while the other groups prolong release to about $1 \mathrm{~h}$ (marked by vertical dash line).

For better clarity, the release profiles of samples anodized at different voltages are shown separately in Figure 4.5. It is seen that for all groups, the amount of loaded drug increases as the anodization duration is increased. The comparison with Figure 4.3 shows that nanotubes with higher aspect ratio release their intercalated drug molecules at slower rate. The effect of the aspect ratio on the drug release rate seems to be larger than the effect of length and volume individually. Nanotubes with the aspect ratio above 18 release the loaded drug in about $1 \mathrm{~h}$, while nanotubes with the aspect ratio below 18 release the loaded drug in less than 15 minutes. 

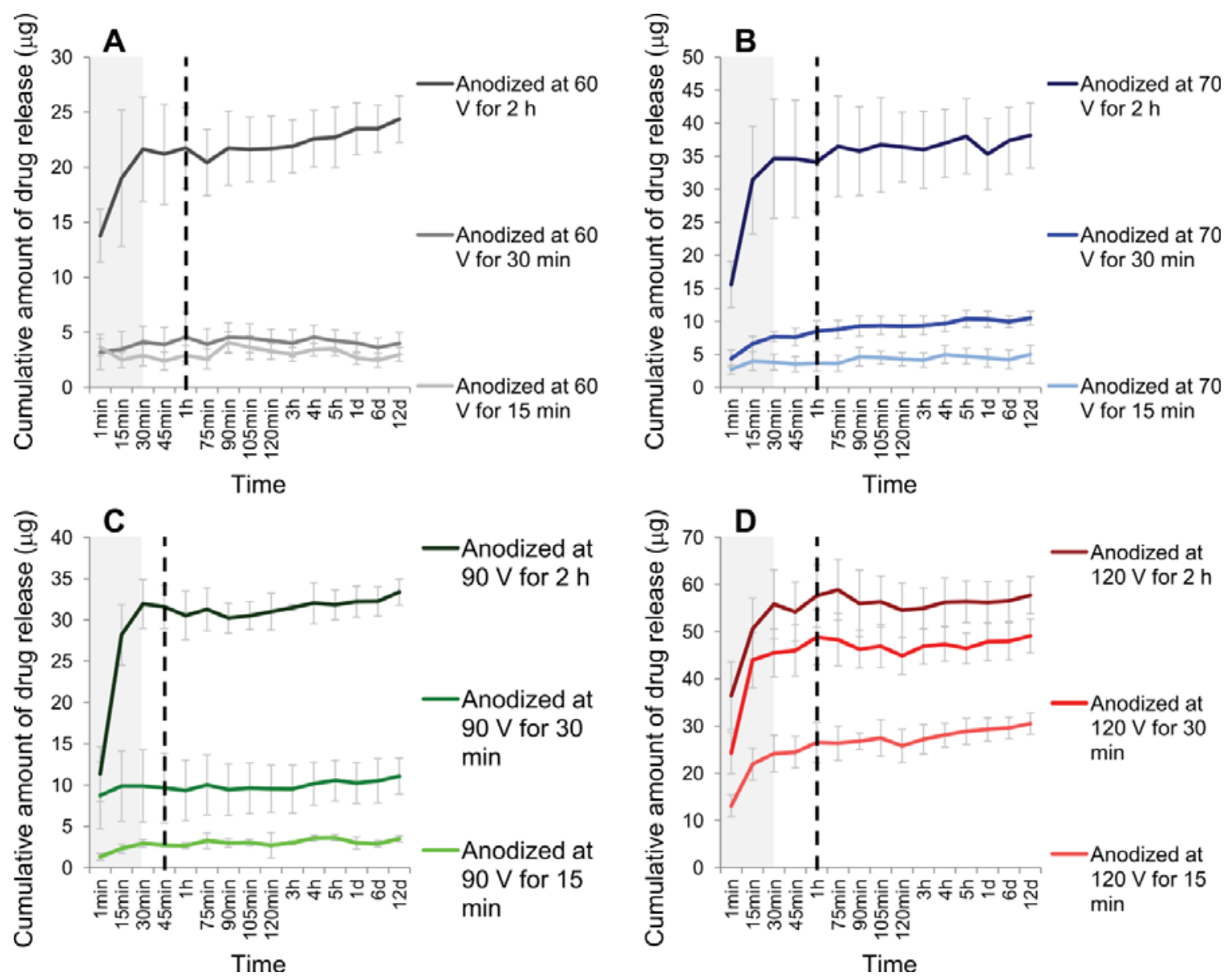

Figure 4.5 Concentration of drug released from TNTs anodized at (A) $60 \mathrm{~V}$, (B) $70 \mathrm{~V}$, (C) $90 \mathrm{~V}$ and (D) $120 \mathrm{~V}$. Shaded domain corresponds to active release stage which lasts for 30 minutes. During this stage, most of the loaded drug is released from nanotubes into aqueous environment. Some groups of TNTs release the overall amount of loaded drug in less than $15 \mathrm{~min}$, while the other groups prolong release to about $1 \mathrm{~h}$ (marked by vertical dash line). Error bars show standard deviation for $\mathrm{n}=3$.

\subsection{Discussion}

Several techniques have been developed to prolong drug release from TNTs. These techniques have promoted osteoblast adhesion, proliferation [155] and differentiation [119] compared with conventional nano-tubular surfaces. In addition, stem cell adhesion and spreading,[156] proliferation, differentiation [157,158] and motogenic responses [158] are enhanced on drug-loaded surfaces, while bacterial adhesion is reduced.[119,159] Many of these methods employ biopolymers to prevent burst release 
of drug by capping TNTs. These methods include covering nano-tubular surface with polymeric layers [118,155,158-160] and intercalation of drug into polymeric micelles $[117,155,160,161]$ followed by absorption of drug-loaded micelles into TNTs. On the other hand, some other techniques have also introduced prolongation such as covering nano-tubular surface with hydroxyapatite via pulsed laser deposition [162] and functionalizing drug onto TNTs.[157] In the present study the effect of TNTs size on prolongation of drug release is demonstrated with the aim of introducing a novel technique which overcomes complications of previously introduced techniques and possible adverse effects caused by application of polymers.

Our results show that some groups of TNTs release loaded drug in a few minutes, while the other groups prolong release to about $1 \mathrm{~h}$ (Figure 4.5). In order to determine the relation between TNT dimensions and the release rate, we specified the role of TNTs diameter (Figure 4.3-A), length (Figure 4.3-B), aspect ratio (Figure 4.3-C), volume (Figure 4.3-D), and the amount of intercalated drug (Figure 4.4-A-D). Table 3.1 shows the relation between these parameters and the measured release rate. Dark cells in the table mark groups of samples with prolonged release, higher diameter, higher length, higher aspect ratio, higher volume and higher amount of intercalated drug. According to Table 3.1, an increase in all these parameters correlates with the prolonged release process. However, the highest correlation is observed for the groups of TNTs with high aspect ratio, for which the release process was the slowest.

A previous study, in agreement with our results, reported that an increase in TNT length results in prolongation of drug release.[91] On the contrary, Yang et al. reported that shorter TNTs are more effective to retard drug release.[118] Based on the data mentioned in these publications, the aspect ratio of the TNTs used can be evaluated. It shows that drug release follows the same trend in both studies. Namely, as the aspect ratio is increased, release is retarded. Another study was performed on the effect of anodic aluminum oxide pore dimensions on the drug release rate. Anodization conditions were controlled to fabricate pores with different diameters and constant length.[161] It is found that pores with smaller diameters are able to prolong drug release.[161] It can be deduced that aspect ratio plays the main role in prolongation of release also in this case. 
There is more variation in length of TNTs fabricated for drug release studies rather than in TNTs diameter. While the range of TNTs lengths varies between $400 \mathrm{~nm}$ $[87,119]$ to $70 \mu \mathrm{m},[105,163]$ the TNTs diameter variation is limited between $60 \mathrm{~nm}[156]$ to $170 \mathrm{~nm}$.[105,163] It explains why the TNTs length has attracted more attention compared with the TNTs aspect ratio. In agreement with our results, loaded drug is reported to be released in short period of time from short TNTs which, in fact, possess low aspect ratio. The entire amount of drug loaded into TNTs with 60-80 nm diameter and short length of less than few micron is released in 25-110 min,[87] 36-105 min [156] and 45-150 min.[119] However, the entire amount of drug loaded into TNTs with 110$170 \mathrm{~nm}$ diameter and 40-70 $\mu \mathrm{m}$ length is released in 4-11 days.[105,117,155,160,163,164] The second group possesses TNTs with higher aspect ratio formed on a smooth surface. However, TNTs with $100 \mathrm{~nm}$ diameter and $500 \mathrm{~nm}$ length formed on a rough substrate also prolonged release to 7 days.[165] TNTs with length of 2.3-6.5 $\mu \mathrm{m}$ were also reported to prolong release for few months, while a vacuum impregnation technique is used for deep intercalation of drug into TNTs.[91]

Table 4.1- Relation between drug release duration and TNT dimensions.

\begin{tabular}{|c|c|c|c|c|c|c|c|c|c|c|c|c|}
\hline & \multicolumn{12}{|c|}{ Anodization condition (voltage and duration) } \\
\hline & \multicolumn{3}{|c|}{$60 \mathrm{~V}$} & \multicolumn{3}{|c|}{$70 \mathrm{~V}$} & \multicolumn{3}{|c|}{$90 \mathrm{~V}$} & \multicolumn{3}{|c|}{$120 \mathrm{~V}$} \\
\hline & $15 \mathrm{~m}$ & $30 \mathrm{~m}$ & $2 \mathrm{~h}$ & $15 \mathrm{~m}$ & $30 \mathrm{~m}$ & $2 \mathrm{~h}$ & $15 \mathrm{~m}$ & $30 \mathrm{~m}$ & $2 \mathrm{~h}$ & $15 \mathrm{~m}$ & $30 \mathrm{~m}$ & $2 \mathrm{~h}$ \\
\hline $\begin{array}{l}\text { Prolonged released } \\
(\geq 30 \mathrm{~min})\end{array}$ & & & & & & & & & & & & \\
\hline $\begin{array}{l}\text { High aspect ratio } \\
(\geq 18)\end{array}$ & & & & & & & & & & & & \\
\hline $\begin{array}{l}\text { High length } \\
(\geq 4 \mu \mathrm{m})\end{array}$ & & & & & & & & & & & & \\
\hline $\begin{array}{l}\text { High volume } \\
\left(\geq 0.09 \mu^{3}\right)\end{array}$ & & & & & & & & & & & & \\
\hline $\begin{array}{l}\text { High amount of } \\
\text { intercalated drug } \\
(\geq 10 \mu \mathrm{g})\end{array}$ & & & & & & & & & & & & \\
\hline $\begin{array}{l}\text { High diameter } \\
(\geq 206 \mathrm{~nm})\end{array}$ & & & & & & & & & & & & \\
\hline
\end{tabular}

Amount of the intercalated drug can also affect release profile. According to previous studies release of drug is sustained from the nanotubes fabricated with the same 
dimensions but loaded with a higher amount of drug. $[87,119,156]$ In the present work, TNTs with higher volume were loaded with higher amount of drug (Table 4.1). Most of the samples from this group show retarded drug release. The other parameters that prolong release process include low temperature,[166] positive electrostatic charge of drug [87] and large size of drug particles.[167] Model drug used in the present study, naproxen sodium, has low molecular weight of 252 Da. Thus, small size of drug molecules can be a reason of the relatively fast release observed in our study. This burst initial release is also correlated to fast diffusion of the drug molecules as a result of high concentration gradient between the drug interface and the PBS solution. The cumulative amount of released drug from $2 \mathrm{~h}$ anodized samples is approximately $40 \mu \mathrm{g}$, which is sufficient to provide a high local concentration of drug during the early few hours subsequent to the orthopedic surgery.

In general, our results suggest that drug-loading capacity and release profile are tuned by controlling nanotube morphology through anodization. In addition, application of the designed system is not limited to any specific kind of drug. In fact, various types of drugs, including growth factors and antibiotics and their mixtures can be loaded into TNTs.

Assume that diffusion of drug in PBS follows one-dimensional Fick's law, and drug initially fills the entire bore of a TNT. Then the volume of the drug inside TNT V diminishes in time as

$$
\frac{\mathrm{dV}}{\mathrm{dt}}=-\frac{\mathrm{D} \Delta \mathrm{c}}{\mathrm{L}} \pi \mathrm{a}^{2}
$$

where $\mathrm{D}$ is the diffusion coefficient of drug molecules in PBS, $\Delta \mathrm{c}=\mathrm{c}_{\text {over surface, eq }}-\mathrm{c}_{\text {out eq }}$, with $\mathrm{c}_{\text {over surface, }}$ being the equilibrium concentration of drug over its solid surface in solution and $\mathrm{c}_{\text {out,eq }}$ being the dimensionless equilibrium concentration of drug molecules in the bath (far from the TNT opening), a is the TNT cross-sectional radius, and L is the current location of the drug dissolution front from the TNT opening.

The geometric considerations provide the following complementary equation for the release rate

$$
\frac{d V}{d t}=-\pi a^{2} \frac{d L}{d t}
$$


Combining Eqs. (1) and (2), we obtain a differential equation for L. Its integration yields

$$
\mathrm{L}=[2 \mathrm{D} \Delta \mathrm{ct}]^{1 / 2}
$$

The latter equation shows that $L \sim \sqrt{t}$. Therefore, according to Eqs. (1) and (3), the drug volume inside TNT decreases as

$$
\mathrm{V}=\mathrm{V}_{0}-[2 \mathrm{D} \Delta \mathrm{ct}]^{1 / 2} \pi \mathrm{a}^{2}
$$

Accordingly, the drug mass released in time $\mathrm{M}_{\text {released }}$ increases as $\sqrt{\mathrm{t}}$, namely

$$
\mathrm{M}_{\text {released }}=\rho \pi \mathrm{a}^{2}[2 \mathrm{D} \Delta \mathrm{ct}]^{1 / 2}
$$

where $\rho$ is the drug density, and thus, the modified drug concentration in the bath also increases as $\sqrt{t}$.

It is emphasized that according to Eq. (3), the complete release duration $t_{\text {release }}$ is given by

$$
\mathrm{t}_{\text {release }}=\frac{\mathrm{L}_{\mathrm{t}}^{2}}{2 \mathrm{D} \Delta \mathrm{c}}
$$

i.e. strongly depends on the TNT length $\mathrm{L}_{\mathrm{t}}$, and does not depend on its cross-sectional radios a. Since according to Eq. (6) the dependence of the release duration on the TNT length $L_{t}$ is quadratic, it is plausible to assume that it determines a strong dependence of the release duration on the TNT aspect ratio.

The experimental data for the active release stage from Figs. 4 and 5 were recalculated per single TNT, separated in Fig. 6 and used for comparison with the theoretical predictions. 


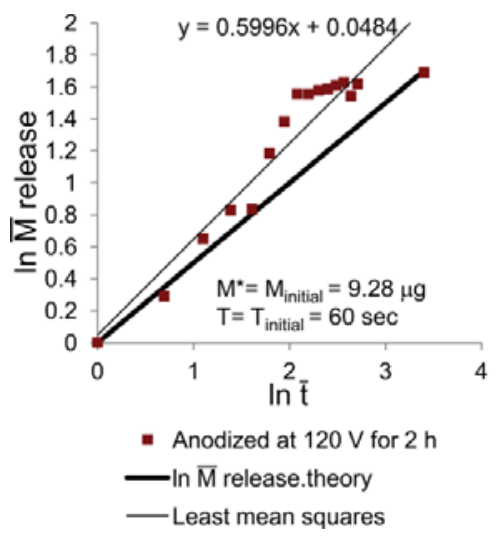

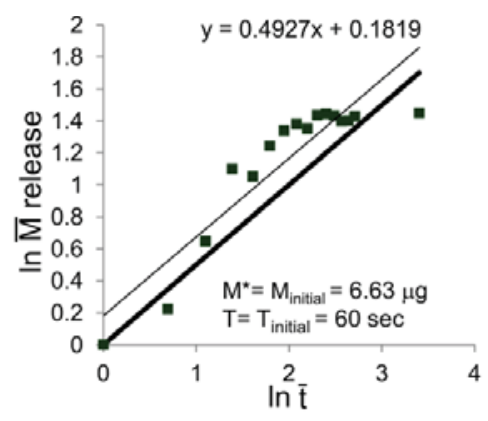

- Anodized at $90 \mathrm{~V}$ for $2 \mathrm{~h}$

- $\ln \overline{\mathrm{M}}$ release.theory

- Least mean squares

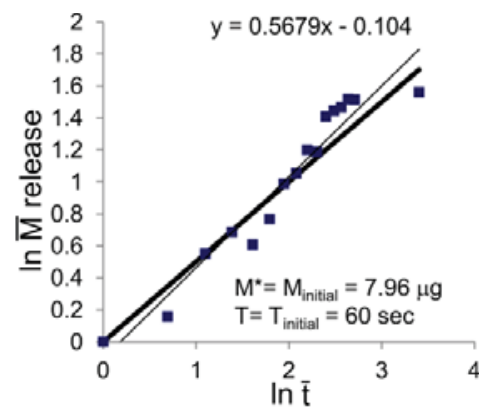

- Anodized at $70 \mathrm{~V}$ for $2 \mathrm{~h}$

- $\ln \overline{\mathrm{M}}$ release.theory

- Least mean squares

Figure 4.6 Experimental data for release from a single TNT versus theoretical predictions. The dimensionless release mass $\bar{M}$ was rendered dimensionless using as scales masses $M_{*}$ listed in the panels. The dimensionless time $\bar{t}$ was rendered dimensionless using as a scale $T_{\text {init }}=60 \mathrm{~s}$. The experimental data are shown by symbols. They were spanned by the thin straight lines using the least-mean-square approximation. The solid straight lines correspond to fitting the theoretical result Eq. (5).

The slopes of the thin straight lines reveal the following exponent values in the scaling dependence of the release amount of drug on time: 0.5996 (for the anodization at $120 \mathrm{~V}$ for $2 \mathrm{~h}$; left in Fig. 6), 04927 (for the anodization at $90 \mathrm{~V}$ for $2 \mathrm{~h}$; middle in Fig. 6), 0.5679 (for the anodization at $70 \mathrm{~V}$ for $2 \mathrm{~h}$; right in Fig. 6). For the comparison, the theoretical scaling exponent revealed by Eq. (5) is $1 / 2$, which shows good agreement with the data. Moreover, the theoretical predictions of Eq. (5) fit the data well at the values of $\mathrm{D} \Delta \mathrm{c}=2.82 \times 10^{-13}$ (for the thick straight line on the left in Fig. 6), $\mathrm{D} \Delta \mathrm{c}=1.88 \times 10^{-13}$ (for the thick straight line in the middle in Fig. 6), and $\mathrm{D} \Delta \mathrm{c}=2.32 \times 10^{-13}$ (for the thick straight line on the right in Fig. 6). Taking for the estimates the values of the drug diffusion coefficient in the bath as $\mathrm{D}=10^{-5} \mathrm{~cm}^{2} / \mathrm{s}$ or $\mathrm{D}=10^{-6} \mathrm{~cm}^{2} / \mathrm{s}$, we obtain the values of $\Delta \mathrm{c}$ listed in Table 4.2. Since $\mathrm{c}_{\text {out,eq }} \approx 0, \Delta \mathrm{c} \approx \mathrm{c}_{\text {over surface, eq }}$. 
Table 4.2- Obtain the values of $\Delta \mathrm{c}$ and $\mathrm{c}_{\text {drug over surface, eq }}^{*}$

\begin{tabular}{|c|c|c|c|}
\hline & $\begin{array}{c}\Delta \mathbf{c} \text { for } \\
\mathbf{D}=\mathbf{1 0}^{-5} \mathbf{c m}^{2} \mathbf{s}^{-\mathbf{1}}\end{array}$ & $\begin{array}{c}\Delta \mathbf{c} \text { for } \\
\mathbf{D}=\mathbf{1 0}^{-\mathbf{6}} \mathbf{c m}^{2} \mathbf{s}^{-\mathbf{1}}\end{array}$ & $\begin{array}{c}\mathbf{c}^{*} \text { drug over surface, eq } \\
\text { for } \mathbf{D}=\mathbf{1 0}^{-5} \mathbf{c m}^{\mathbf{2}} \mathbf{s}^{-\mathbf{1}}\end{array}$ \\
\hline $\mathbf{( 1 2 0} \mathbf{V})$ & $2.82 \times 10^{-8}$ & $2.82 \times 10^{-7}$ & 0.035282 \\
\hline $\mathbf{( 9 0} \mathbf{V})$ & $1.88 \times 10^{-8}$ & $1.88 \times 10^{-7}$ & 0.023579 \\
\hline $\mathbf{( 7 0} \mathbf{V})$ & $2.32 \times 10^{-8}$ & $2.32 \times 10^{-7}$ & 0.029041 \\
\hline
\end{tabular}

The equilibrium dimensional drug concentration over the surface of solid drug in TNT is, thus equal to $c_{\text {drug over surface, eq }}^{*}=\rho \Delta c$. Taking the solid drug density $\rho=1.25 \mathrm{~g} / \mathrm{cm}^{3}$, we find the values of $\mathrm{c}_{\text {drug over surface, eq }}^{*}$ listed in Table 4.2.

\subsection{Conclusions}

Our results suggest that drug release from titania nanotubes (TNT) is a diffusionlimited process. Its duration is strongly affected by the TNTs aspect ratio. An increase in TNTs diameter, length, aspect ratio, volume, and the amount of intercalated drug correlates with the prolonged release process. However, the highest correlation is observed for the groups of TNTs with high aspect ratio, for which the release process was the slowest. Considering that enhancing TNTs length is harmless for cells, increasing TNTs aspect ratio via enhancing TNTs length can be a promising technique for prolonging drug release while bioactivity of loaded drugs is protected as well.

\subsection{Methods}

Fabrication of TNTs

Ti90Al6V4 alloy (Ti-V) disks with $15 \mathrm{~mm}$ diameter and $1 \mathrm{~mm}$ thickness were used in this study. Disks were polished prior to anodization using a series of silicon carbide grinding papers (\#320, \#400, \#600, and \#800) (Carbimet 2, Buehler, Lake Bluff, IL, USA), followed by polishing cloth (TexMet Polishing Cloth, Beuhler) with diamond paste (MetaDi 9-micron, Beuhler) and lubricant (MetaDi Fluid, Beuhler). Chemomet polishing cloth (Chemomet I, Buheler) with colloidal silica polishing suspension (MasterMed, Buehler) was used as a final polishing procedure in order to obtain a mirror- 
like surface. To remove polishing agents, disks were sonicated in acetone (Fisher) for 30 min, rinsed in DI water and dried in air.

In order to fabricate TNTs on the smooth surface trough anodization, samples were attached to a DC voltage source (Keithley 2400 SourceMeter) as the working electrode while copper rod was used as the counter-electrode. Both electrodes were immersed in electrolyte of ethylene glycol (Fisher) solution containing $0.2 \mathrm{wt} \% \mathrm{NH}_{4} \mathrm{~F}$ (Fisher) and $4 \mathrm{wt} \%$ DI water. A magnetic stirrer was used to agitate the electrolyte as DC voltage was applied for specific duration. Anodization was performed at room temperature.

In order to obtain TNTs with different aspect ratios, anodization was conducted at different voltages of $60 \mathrm{~V}, 70 \mathrm{~V}, 90 \mathrm{~V}$ and $120 \mathrm{~V}$. At each voltage, anodization was performed for $15 \mathrm{~min}, 30 \mathrm{~min}$ and $2 \mathrm{~h}$. For each specific anodization voltage and duration 3 disks were anodized.

\section{Scanning Electron Microscopy:}

Structural characterization of the prepared TNTs was performed using a field emission scanning electron microscope (FESEM), JEOL JSM-6320F. In order to image dimensions of TNTs and determine their aspect ratio, TNTs were removed from substrate by scratching. Detached particles were placed on a double-sided conductive carbon tape and attached to an aluminum s for imaging. Finally ImageJ software was used to measure dimensions of nanotubes. Measurement was done 3 times.

\section{Intercalation of drug molecules}

To intercalate drug into TNTs, the method of self-sustained diffusion was used.[154,168-170] To make sure anodization residues are removed prior to drug loading, disks were sonicated in acetone (Fisher) for $30 \mathrm{~min}$, rinsed in DI water and dried in air. Naproxen sodium, an anti-inflammatory drug, was used in this study as a model drug. Naproxen sodium is fully soluble in water at neutral $\mathrm{pH}$. It has molecular weight of 252.23 $\mathrm{Da}$ and molecular formula $\mathrm{C}_{14} \mathrm{H}_{13} \mathrm{NaO}_{3}$. In order to intercalate naproxen sodium, $2 \mathrm{ml}$ solution of drug in ethanol with concentration of $2 \mathrm{mg} / \mathrm{ml}$ was prepared for each sample. Prepared drug solution was pipetted on nano-tubular surface during 20 times of 
pipetting. At each time, $100 \mu \mathrm{l}$ of solution was pipetted and allowed to dry under hood. As the solvent evaporated, drug penetrated into TNTs. Then dried surface was wiped with soft tissue to remove excess drug. After wiping, pipetting was repeated to load substantial amount of drug. After last loading cycle, surface wiping was followed by quick rinsing in PBS. Finally disks were quickly dried by exposing to compressed air before performing release measurements.

Transmission electron microscopy and Energy-dispersive X-ray spectroscopy

Visualization of the intercalated samples was performed using a Transmission Electron Microscope (TEM) JEOL JEM 3010. The TEM images and corresponding Energy-Dispersive X-ray Spectroscopy (EDS) analysis of pristine TNTs are presented in figure 4.2 .

\section{Measuring drug release}

Drug-loaded disks were immersed in $2 \mathrm{ml}$ pure PBS to release the drug. At specific time instants, $100 \mu$ l of solution was pipetted out to measure the amount of released drug, followed by pipetting in $100 \mu$ of pure PBS to keep the solution volume constant. UV-Vis photospectroscopy (Thermo Scientific, Nanodrop $1000 \mathrm{UV} / \mathrm{Vis}$ Spectrophotometer) was used at the wavelength of $320 \mathrm{~nm}$ to measure the absorbance of drug solution in phosphate buffer solution (PBS).

A calibration curve was established by measuring the absorbance of drug solutions with known concentrations. This calibration curve was used to determine concentration of drug based on the measured absorbance values for solutions of released drug. Since $5 \%$ of drug-containing solution is replaced with $5 \%$ fresh PBS as each measurement was done, the modified concentration was calculated and used.

\section{Acknowledgements}

Authors would like to thank Michigan Technological University for providing the funding to support this research. The authors are grateful to Prof. Craig Friedrich, director of Multiscale Technologies Institute at Michigan Technological University for 
the discussions, to Prof. William Hendrickson, UIC, Research Resource Center, for providing the laboratory space for conducting the experiments, to Mr. Canhui Wang for help with TEM imaging, and to Mr. Justin Feng for his assistance in conducting experiments. 


\title{
Chapter 5
}

\section{Enhancing Surface Characteristics of Ti-6Al-4V for Bio-implants Using Integrated Anodization and Thermal Oxidation*}

\author{
Sweetu B. Patel ${ }^{\mathrm{a}}$, Azhang Hamlekhan ${ }^{\mathrm{b}}$, Dmitry Royhman ${ }^{\mathrm{c}}$, Arman Butt ${ }^{\mathrm{a}}$, Judy Yuan ${ }^{\mathrm{c}}$, \\ Tolou Shokuhfar, ${ }^{\mathrm{b}, \mathrm{d}}$, Cortino Sukotjo ${ }^{\mathrm{c}}$, Mathew T. Mathew ${ }^{\mathrm{e}}$, Gregory Jursich ${ }^{\mathrm{a}}$, Christos \\ G. Takoudis ${ }^{\mathrm{a}, \mathrm{f}, \mathrm{g}}$ \\ ${ }^{a}$ Department of Bioengineering, University of Illinois at Chicago, Chicago, Illinois \\ 60607, USA \\ ${ }^{b}$ Department of Mechanical Engineering, Michigan Technological University, \\ Houghton, Michigan 49931, USA \\ ${ }^{c}$ Department of Restorative Dentistry, University of Illinois at Chicago, Chicago, \\ Illinois 60612, USA \\ ${ }^{d}$ Department of Physics, University of Illinois at Chicago, Chicago, Illinois 60612, \\ USA \\ ${ }^{e}$ Department of Orthopaedic Surgery, Rush University Medical Center, Chicago, \\ Illinois 60612, USA \\ ${ }^{f}$ Department of Chemical Engineering, University of Illinois at Chicago, Chicago, \\ Illinois 60607, USA \\ ${ }^{g}$ Corresponding author
}

*The material contained in this chapter has been published in the open access Journal of Materials Chemistry B. My contribution to this publication includes fabrication of nanotubes, FESEM and reviewing the manuscript. 


\begin{abstract}
Modifications of Ti-6Al-4V surface roughness, wettability and composition are increasingly studied to improve cellular viability on biomedical implants involving Ti$6 \mathrm{Al}-4 \mathrm{~V}$. In this study, it is shown that modification of Ti-6Al-4V samples using anodization (for the formation of titania nanotubes) combined with thermal oxidation (TO) results in superior surface characteristics to those of a smooth, rough, anodizedsmooth or anodized-rough surface alone. Surface characterization is performed using water contact angle (WCA) measurements, white-light interferometry, Fourier transform infrared spectroscopy (FTIRS), field emission scanning electron microscopy and grazing incidence x-ray diffraction (GIXRD). WCA measurements before TO indicate that anodized-smooth and anodized-rough samples are super-hydrophilic (WCA less than $5^{\circ}$ ); WCA of non-anodized smooth and rough surfaces are $57 \pm 6^{\circ}$ and $86 \pm 7^{\circ}$, respectively. After TO at $450{ }^{\circ} \mathrm{C}$ for 3 hours, all samples become super-hydrophilic; however, three weeks after TO, smooth and rough surfaces become hydrophobic, while anodized-smooth and anodized-rough surfaces remain hydrophilic. FTIRS and GIXRD data show that the TO of anodized and non-anodized smooth samples results in anatase and rutile $\mathrm{TiO}_{2}$, of which anatase is favorable for cellular attachment. Micro-/nano-scale roughness and TO are discussed in the context of enhancing Ti-6Al-4V surface characteristics.
\end{abstract}

\title{
5.1 Introduction
}

The Ti-6Al-4V alloy is widely used as an implant material in orthopedics and dentistry; it is an ideal material for implants due to its high biocompatibility, high mechanical resistance, low density, high corrosion resistance, high chemical inertness, and atoxicity. ${ }^{[6,171-176]}$ Though Ti-6Al-4V possesses the required properties for implants, over time these implants fail; one of the major causes of implant failure include improper bone-implant interaction. Some of the factors that influence osseointegration include surface composition, surface free energy (SFE), and surface roughness. ${ }^{[139,173,177-184]}$ 
In order to prolong effective lifetime of implant materials, it is essential to further improve the corrosion resistance of the exposed implant material against undesired biochemical reactions of Ti-6Al-4V in contact with acidic, basic or any other bodily fluid present at the implant site. Modification of Ti-6Al-4V surfaces with a $\mathrm{TiO}_{2}$ layer can enhance the aforementioned properties required for implants because such a layer serves as a passive layer, improves surface wettability, and it enhances cellular response through improved bone morphogenic protein (BMP) signaling, apatite formation, and upregulation of collagen II, osteocalcin, and biglycan. ${ }^{[180-187]}$ Some of the previously established methods to modify a metal surface with $\mathrm{TiO}_{2}$ include chemical vapor deposition (CVD), physical vapor deposition (PVD), sol-gel, atomic layer deposition (ALD), sandblasting and acid etching (roughening), and thermal oxidation (TO). [139,172,176,188-192] Deposition of $\mathrm{TiO}_{2}$ using ALD provides a uniformal and conformal coating with thickness control down to atomic layer; however, it is a time consuming technique for micron-level or thicker film formation as it often has a growth rate of $\sim 0.1$ $\mathrm{nm} / \mathrm{min}$, it requires expensive precursors and vacuum equipment, and it has high processing cost. ${ }^{[193,194]} \mathrm{CVD}$, on the other hand, can have a growth rate of $10 \mathrm{~nm} / \mathrm{min}$, which is 100 times faster than ALD; however, it has limited step coverage, strict working temperature range and pressure constraints, and high maintenance cost. ${ }^{[195]}$ PVD has similar growth rates to that of CVD; however, it is restricted to line of sight coating and thus has poor step coverage with vacuum requirements and high maintenance cost.

In this study, a simpler, lower processing cost approach of thermal oxidation is used to form a $\mathrm{TiO}_{2}$ layer on Ti-6Al-4V surfaces. $\mathrm{TO}$ can achieve growth rates similar to those of CVD and PVD. However, unlike ALD, CVD and PVD, TO does not require expensive precursor reagents; instead it is based on diffusion and reaction of oxygen in the metal alloy substrate. ${ }^{[196-199]}$ In TO, the diffusion rate of oxygen is the rate limiting factor and it depends on temperature and time. ${ }^{[199]}$ At room temperature, oxygen readily reacts with titanium forming native oxide layer less than $10 \mathrm{~nm}$-thick, while at higher temperatures up to $1200{ }^{\circ} \mathrm{C}$, it can result in a much thicker oxide layer on the order of $10 \mathrm{~s}$ of $\mu$ m-thick within hours. ${ }^{[139,199-204]}$ Therefore, TO is the preferred method for $\mathrm{TiO}_{2}$ thin films in this study. 
Electrochemical anodization of titanium produces $\mathrm{TiO}_{2}$ nanotubes (TNTs), which can improve the cellular response of human mesenchymal stem cells (hMSCs) through more favorable surface morphology and increased surface area available for cellular adhesion. ${ }^{[6,205]}$ TNTs are created through anodization in an electrochemical cell with various electrolyte composition including fluorine-containing ones [6,167,206-219] Anodization of Ti-6Al-4V using ammonium fluoride and ethylene glycol results in a surface composition of $\mathrm{Ti}(\mathrm{OH})_{4}$, which can be converted into super-hydrophilic crystalline structured $\mathrm{TiO}_{2}$ by thermal oxidation at certain temperatures. ${ }^{[192,218]}$ The diameter and length of nanotubes can be controlled by the applied anodization voltage, electrolyte wt. \% composition of fluoride, and time duration of anodization. ${ }^{[6,167,220]}$

Thus far, only one group, D.H. Shin et al., has performed an experiment with similar experimental conditions to this study. The main objective of their study was to investigate the surface wettability of anodized and non-anodized commercially pure $\mathrm{Ti}$ and Ti-6Al-4V samples before and after thermal oxidation treatment and after aging, whereas the main objective of our study is to obtain the optimal parameters for Ti-6Al$4 \mathrm{~V}$ sample treatment in terms of surface wettability, composition and roughness ${ }^{[218]}$; Study by D.H. Shin et al. was different than our study in several ways, i) their surface wettability results show similar trend yet they are significantly different compared to the results obtained in this study. For example, their WCA of anodized Ti-6Al-4V samples before and after annealing were $32^{\circ}$ and $14^{\circ}$, respectively, whereas in our study, WCA of less than $5^{\circ}$ was obtained pre and post annealing ii) thorough surface composition and crystallinity analysis is lacking for before and after TO (i.e. deconvolution of Fourier transform infrared spectroscopy (FTIRS) and grazing incident X-ray diffraction spectra (GIXRD) analysis) and iii) analysis on the effect of annealing on each surface using white light interferometry is not performed, which can be useful in determining the integrity of substrate of interest. Therefore, a comprehensive approach with extensive analysis that addresses the surface characteristics (i.e. wettability, roughness, composition and crystallinity) of smooth, rough, anodized-smooth and anodized-rough Ti-6Al-4V samples before and after TO is still lacking. Both smooth and rough surfaces were investigated in this study, because one of the factors that effect surface wettability 
behavior is surface roughness. Main objectives of this study are 1) to investigate the surface roughness of smooth, anodized-smooth, rough and anodized-rough samples before and after TO using white-light interferometry, 2) to probe the surface composition and crystallinity before and after TO using FTIRS and GIXRD, respectively, and 3) to examine the surface wettability of the aforementioned Ti-6Al-4V samples with surface roughness and surface composition at different stages of the experiment (Table 5.1). 
Table 5.1: Steps of experiment when WCA measurements were performed. BW $=$ Before Wash, AW = After Wash

\begin{tabular}{|c|c|c|}
\hline Steps & Labels & Description \\
\hline 1 & As-prepared & $\begin{array}{c}\text { As-prepared samples were rinsed with DI water } \\
\text { followed by } \mathrm{N}_{2} \text { drying before WCA } \\
\text { measurement }\end{array}$ \\
\hline 2 & BW after aging for 29 days & $\begin{array}{c}\text { WCA was monitored before TO. Samples were } \\
\text { aged for } 29 \text { days. WCA measurement was } \\
\text { obtained before DI water wash }\end{array}$ \\
\hline 3 & AW & $\begin{array}{c}\text { WCA was monitored before TO. Samples were } \\
\text { aged for } 29 \text { days. WCA measurement was } \\
\text { obtained after DI water wash }\end{array}$ \\
\hline 4 & Immediately after TO & TO was performed at $450{ }^{\circ} \mathrm{C}$ for 3 hours \\
\hline 5 & 3 weeks after TO & $\begin{array}{c}\text { WCA was monitored after TO. Samples were } \\
\text { aged for } 3 \text { weeks. WCA measurement was } \\
\text { obtained before DI water wash }\end{array}$ \\
\hline
\end{tabular}

\subsection{Results and Discussion}

Field Emission Scanning Electron Microscopy Analysis

Field emission scanning electron microscopy (FE-SEM) images of the sample surfaces before TO are shown in Figure 5.1. Figures 5.1a and 5.1b show the surface topography of smooth and rough Ti-6Al-4V samples, respectively. The morphology of the rough sample shows major grooves on the surface due to the sandblasting procedure; within those major grooves there are finer grooves, which have been apparently formed by the acid etching procedure (Figures 5.1b). ${ }^{[221]}$ These processing steps result in additional surface area on the samples. Figures 5.1c and 5.1d show the surface topography of anodized-smooth and anodized-rough surfaces, respectively. TNTs are visible on the anodized surface along with some nanograss formation. After 3 or more hours of anodization, it was observed that TNTs are converted into unstable nanograss likely due to the etching of the top of the TNTs. Anodized-smooth samples only have nano-level roughness due to the presence of nanotubes; however, anodized-rough samples have both micro and nano-level roughness perhaps because of the presence of nanotubes on the initially rough surface resulting from sandblasting and acid etching. 

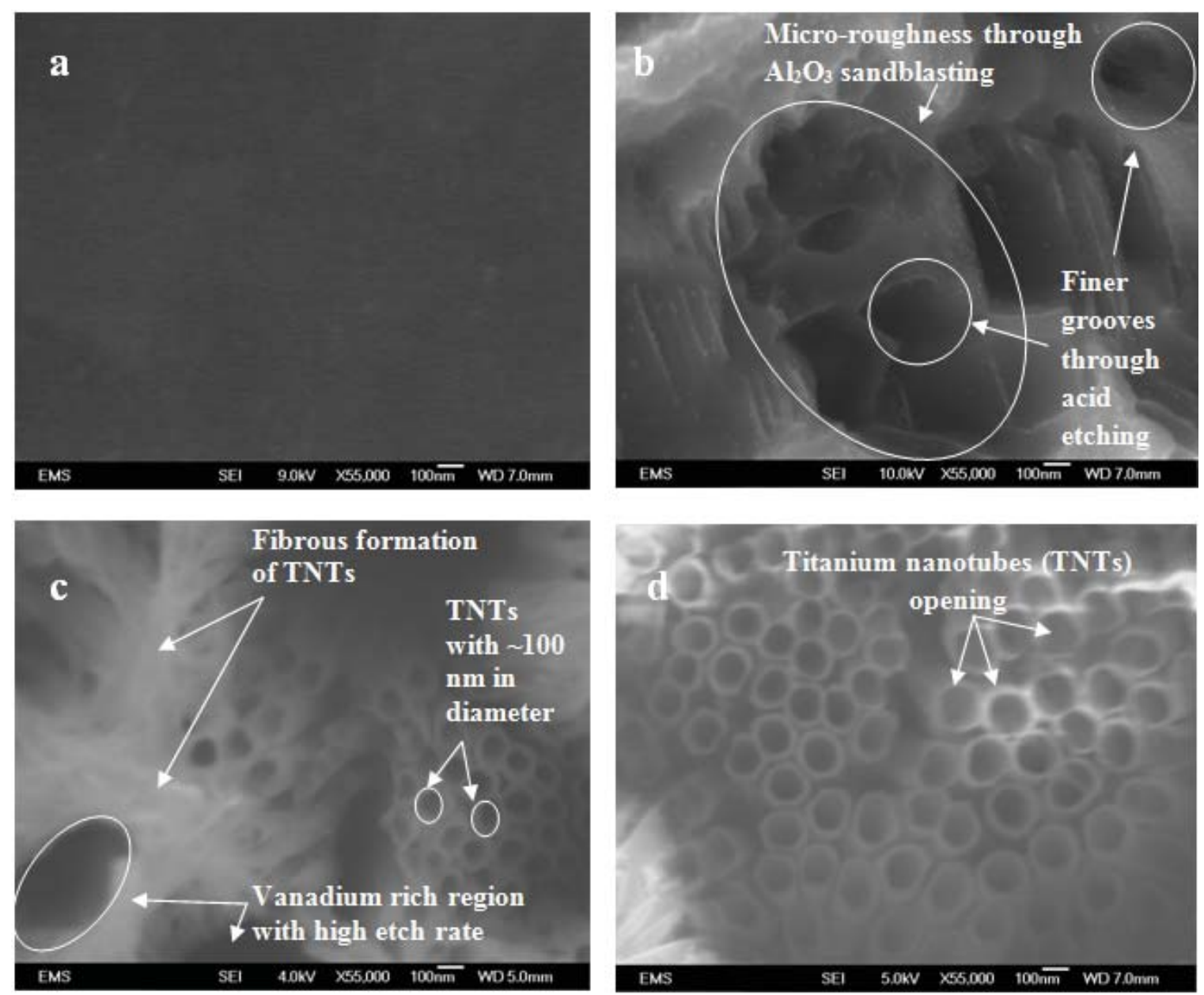

Figure 5.1 FE-SEM images of Ti-6Al-4V samples before TO with X55k magnification and $100 \mathrm{~nm}$ scale a) smooth, b) rough (sandblasted with $\mathrm{Al}_{2} \mathrm{O}_{3}$, and acid etched in $\mathrm{H}_{2} \mathrm{SO}_{4}$ and $\mathrm{H}_{2} \mathrm{O}_{2}$ ), c) anodized-smooth, and d) anodized-rough; Anodization was performed in a solution mixture of EG, $\mathrm{NH}_{4} \mathrm{~F}$, and $\mathrm{H}_{2} \mathrm{O}$ at $60 \mathrm{~V}$ for 4 hours.

The dark regions in the FE-SEM images of the anodized samples (Figures 5.1c, 5.1d) are attributed to vanadium-rich regions subjected to accelerated etching. ${ }^{[222,223]}$ Micro and nano-scale fluctuations on the anodized surfaces may also promote boneimplant integration by providing more surface area for cellular adhesion. ${ }^{[31]}$

Figure 5.2 shows the FE-SEM images of the same samples after TO. The surface morphology of smooth and rough samples before TO (Figures 5.1a and 5.1b) and after TO (Figures 5.2a and 5.2b) shows no significant differences. This corroborates the conformality of TO. Figure 5.2b displays the FE-SEM image of a thermally oxidized 
rough sample, which has apparent irregular micro-grooves. Figure 5.2c shows the FESEM image of thermally oxidized anodized-smooth sample. It is observed that detached nanotubes are covering half of the samples' surface, and some apparently broken nanotubes are found between the highly etched vanadium regions. Figure $5.2 \mathrm{~d}$ shows the FE-SEM image of thermally oxidized anodized-rough surface, where nanotubes are seen to be collapsing. Such collapsing and nanograss formation of TNTs can result from the TO process and prolonged anodization as it is seen in Figure 5.2d. Thermal oxidation of anodized-smooth and anodized-rough samples can crystallize the nanotube walls, which affect the integrity of the TNTs, and may result in nanotube detachment from the substrate. ${ }^{[218]}$ 

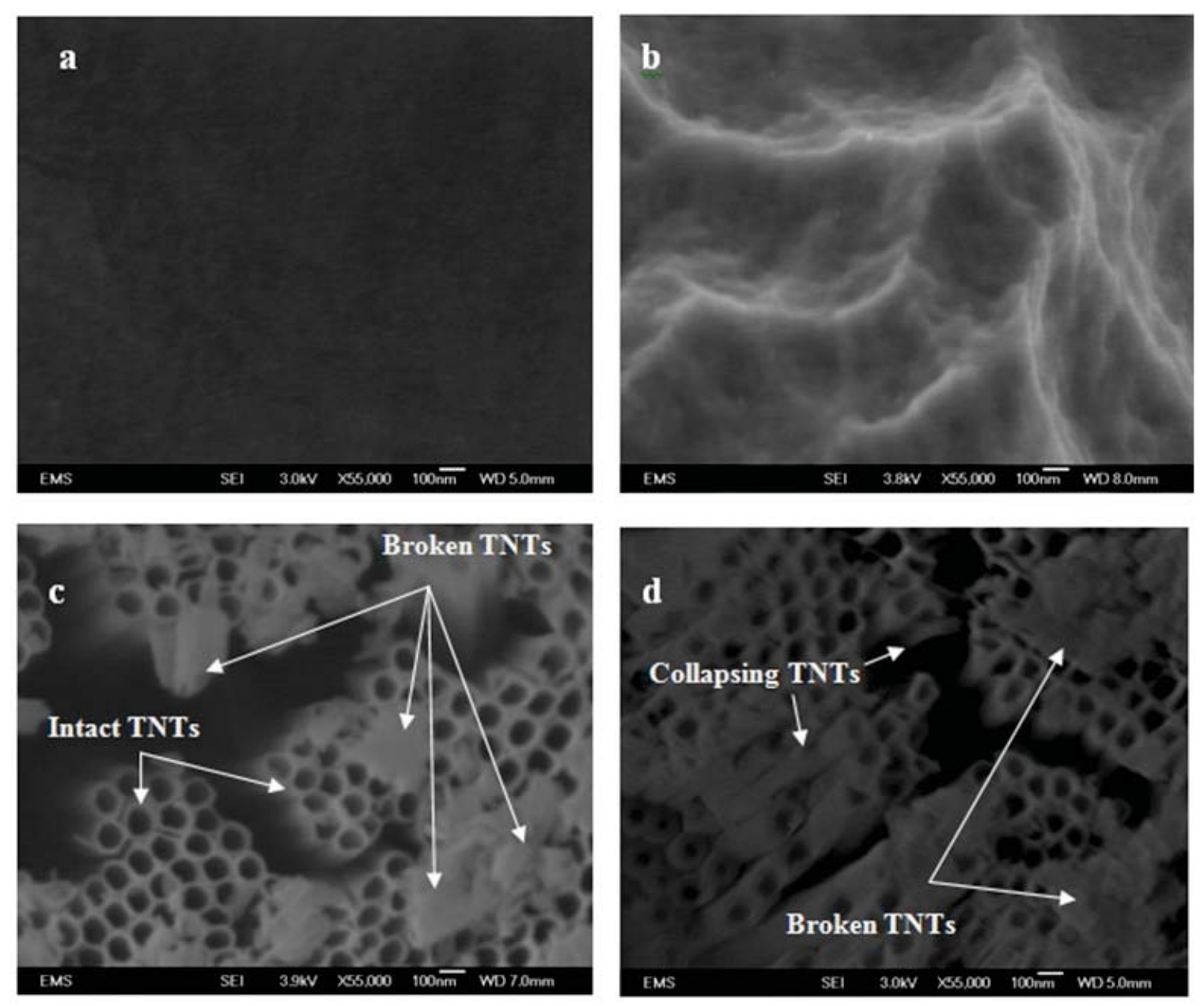

Figure 5.2 FE-SEM images of Ti-6Al-4V samples after TO $\left(450{ }^{\circ} \mathrm{C}, 3 \mathrm{hrs}\right)$ with $\mathrm{X} 55 \mathrm{k}$ magnification and $100 \mathrm{~nm}$ scale a) smooth sample, b) rough sample, c) anodized-smooth sample, and d) anodized-rough sample; Rough and anodized samples were prepared using same condition as that in Figure 5.1.

White-Light Interferometry Analysis

The changes in surface roughness of all samples were probed with white-light interferometry (WLI) before and after TO. In WLI, white light passes through the objective lens on to the samples surface. The objective lens divides light into 2 paths: i) 
that shines on the substrate of interest and ii) that shines on the internal reference surface. Light wave that shines on the substrate cause the measurement wave-front to travel different distances due to surface irregularities than the reference wave-front. This cause interference in the wave-fronts when they are recombined, which is read by a photo-detector and gets translated into a 3D image. The average surface roughness and root mean square roughness of the samples are shown in Table 5.2. Significant differences are found in the surface roughness of smooth, anodized-smooth, rough, and anodized rough samples $(p<0.0001$; one-way ANOVA). Also, the surface roughness within each group of different surface topography before and after thermal oxidation is seen to change sometimes ( $p<0.0001$; Tukey HSD post hoc analysis). For example, after $\mathrm{TO}$, the surface roughness of anodized-smooth and anodized-rough samples is found to decrease likely due to the accumulation of detached nanotubes on the surface or the collapsing of nanotubes. ${ }^{[218]}$ When broken nanotubes gets accumulated on the surface, they block the opening of the intact nanotubes, thereby restricting the WLI light from going inside the nanotubes. WLI light gets reflected from the broken and accumulated nanotube on the surface, which explains the decrease in surface roughness of anodized samples after TO. The surface roughness of smooth samples is seen to slightly increase perhaps due to the crystallization of the $\mathrm{TiO}_{2}$ surface after $\mathrm{TO}$, while the one of rough samples shows no difference before and after annealing, in agreement with results reported by Saldana et al. ${ }^{[224]}$ Increase in smooth surface roughness is seen after TO at $450{ }^{\circ} \mathrm{C}$ because, i) amorphous $\mathrm{TiO}_{2}$ changes to anatase and rutile structures, which are both tetragonal crystals, however with different lattice parameters and ii) $\mathrm{TiO}_{2}$ grain size increases with annealing temperature resulting in rougher surface. ${ }^{[225,226]}$ 
Table 5.2: Quantitative comparison of average surface roughness and root mean square of the samples before and after thermal oxidation $\left(450{ }^{\circ} \mathrm{C}, 3\right.$ hours); a: $p<0.0001$ compared to group 2; A: $p<0.0001$ compared to group AS, R and AR; $\mathrm{B}: p<0.0001$ compared to group R and AR; $\mathrm{C}: p<0.0001$ compared to group AR

\begin{tabular}{|c|c|c|c|c|}
\hline \multirow[t]{2}{*}{ Surface topography } & \multicolumn{2}{|c|}{ Average roughness (Ra) / $\mu \mathrm{m}$} & \multicolumn{2}{|c|}{ Root mean square $(\mathrm{Rms}) / \mu \mathrm{m}$} \\
\hline & Before TO (1) & After TO (2) & Before TO (1) & After TO (2) \\
\hline Smooth (S) & $0.01 \pm 0.003^{\mathrm{a}, \mathrm{A}}$ & $0.02 \pm 0.004^{\mathrm{A}}$ & $0.02 \pm 0.003^{\mathrm{a}, \mathrm{A}}$ & $0.03 \pm 0.01^{\mathrm{A}}$ \\
\hline $\begin{array}{l}\text { Anodized-smooth } \\
\text { (AS) }\end{array}$ & $3.9 \pm 0.3^{\mathrm{a}, \mathrm{B}}$ & $0.6 \pm 0.06^{\mathrm{B}}$ & $5.6 \pm 0.3^{\mathrm{a}, \mathrm{B}}$ & $1.01 \pm 0.2^{\mathrm{B}}$ \\
\hline Rough (R) & $1.7 \pm 0.3^{\mathrm{C}}$ & $1.7 \pm 0.1^{\mathrm{C}}$ & $2.2 \pm 0.2^{\mathrm{C}}$ & $2.1 \pm 0.1^{\mathrm{C}}$ \\
\hline Anodized-rough (AR) & $4.3 \pm 0.4^{\mathrm{a}}$ & $2.2 \pm 0.3$ & $4.9 \pm 0.4^{\mathrm{a}}$ & $3.0 \pm 0.4$ \\
\hline
\end{tabular}

Fourier Transform Infrared Spectroscopy and Grazing Incidence X-ray Diffraction Analysis

FTIRS data of smooth and anodized-smooth samples before and after thermal oxidation at $450{ }^{\circ} \mathrm{C}$ for 3 hours is shown in Figures 5.3 and 5.4, respectively. In both FTIR spectra, gas phase $\mathrm{CO}_{2}\left(2200-2400 \mathrm{~cm}^{-1}\right)$ and $\mathrm{H}_{2} \mathrm{O}\left(3600-3800\right.$ and $1400-1900 \mathrm{~cm}^{-}$ $\left.{ }^{1}\right)$ absorption peaks are observed from residual air in the FTIR purging chamber system. For smooth samples, an intense peak is observed in the $650-1050 \mathrm{~cm}^{-1}$ region after TO (Figure 5.3a), which is due to the oxidation of Ti and Al from the bulk Ti-6Al-4V alloy. This aforementioned region consists of the superposition of various absorption bands. Figure $5.3 \mathrm{~b}$ shows the de-convolution of the region $650-1050 \mathrm{~cm}^{-1}$ indicating the presence of Ti-O bond of anatase and rutile $\mathrm{TiO}_{2}$ at 870 and $830 \mathrm{~cm}^{-1}$, respectively, ${ }^{[139]}$ possible Ti-O-Ti vibration stretch at $820 \mathrm{~cm}^{-1}$ and $\mathrm{Al}_{2} \mathrm{O}_{3}$ at $840 \mathrm{~cm}^{-1}$. [145,227] 

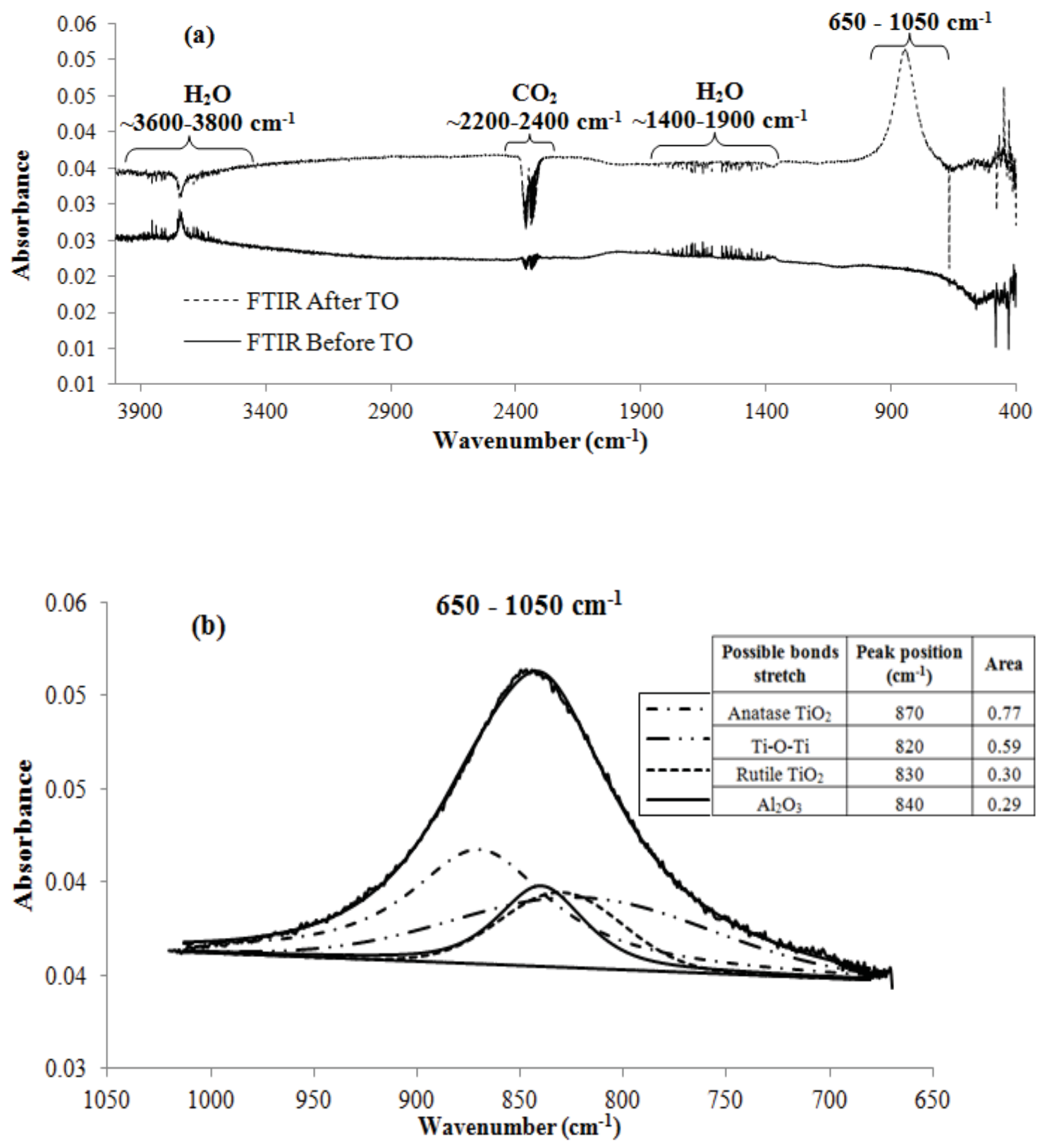

Figure 5.3 FTIR spectra of smooth Ti-6Al-4V samples (a) before (solid line) and after (dashed line) thermal oxidation at $450{ }^{\circ} \mathrm{C}$ for $3 \mathrm{hrs}$ in ambient air; (b) de-convolution of the region $\left(650-1050 \mathrm{~cm}^{-1}\right)$ obtained after TO of smooth Ti-6Al-4V samples. Legend in (b) provides the possible bonds stretch, peak position, and area under the curves. 
Similarly, de-convolution of the anodized-smooth samples' spectra for 1100-1900 and $600-1200 \mathrm{~cm}^{-1}$ region are shown in Figures 5.4b-4e. Some of the infrared features at $1100-1900 \mathrm{~cm}^{-1}$ region are more intense before thermal oxidation (Figure 5.4b) compared to those after thermal oxidation (Figure 5.4d); these peaks are assigned to bonds stretch of $-\mathrm{NH}_{2}$ at $1570.4 \mathrm{~cm}^{-1}, \mathrm{H}-\mathrm{N}-\mathrm{H}$ at $1672.2 \mathrm{~cm}^{-1}, \mathrm{NH}_{4}{ }^{+}$at $1464.1 \mathrm{~cm}^{-1}, \mathrm{Al}-\mathrm{O}$ at $1382 \mathrm{~cm}^{-1}$, $\mathrm{Al}=\mathrm{O}$ at $1367.4 \mathrm{~cm}^{-1}$, and absorbed $\mathrm{NH}_{3}$ on $\mathrm{TiO}_{2}$ at $1239.7 \mathrm{~cm}^{-1}$. ${ }^{[145,228-232]}$ However, features for $\mathrm{H}_{2} \mathrm{O}$ at $1616 / 1624.3 \mathrm{~cm}^{-1}$, adsorbed $\mathrm{NH}_{3}$ on $\mathrm{TiO}_{2}$ at $1198.4 \mathrm{~cm}^{-1}$ and Ti-OH at $1311.5 \mathrm{~cm}^{-1}$ appear to increase after TO in the $1100-1900 \mathrm{~cm}^{-1}$ region. ${ }^{[228,233]}$ After TO, an absorption band resulted at $1755.9 \mathrm{~cm}^{-1}$ could be assigned to either $\mathrm{NH}_{4}{ }^{+}$from the re-deposition of evaporated $\mathrm{NH}_{4}{ }^{+}$from anodization electrolyte or to $\mathrm{CO}_{3}{ }^{2-}$ from the chemical reaction between metal oxides (i.e. $\mathrm{TiO}_{2} / \mathrm{Al}_{2} \mathrm{O}_{3}$ ) and/or hydroxides (i.e. Ti$\mathrm{OH} / \mathrm{Al}-\mathrm{OH})$ with $\mathrm{CO}_{2}$ coming from the environment during TO. ${ }^{[234-236]}$ These same features are also seen to increase and/or decrease for anodized-rough samples after TO. 

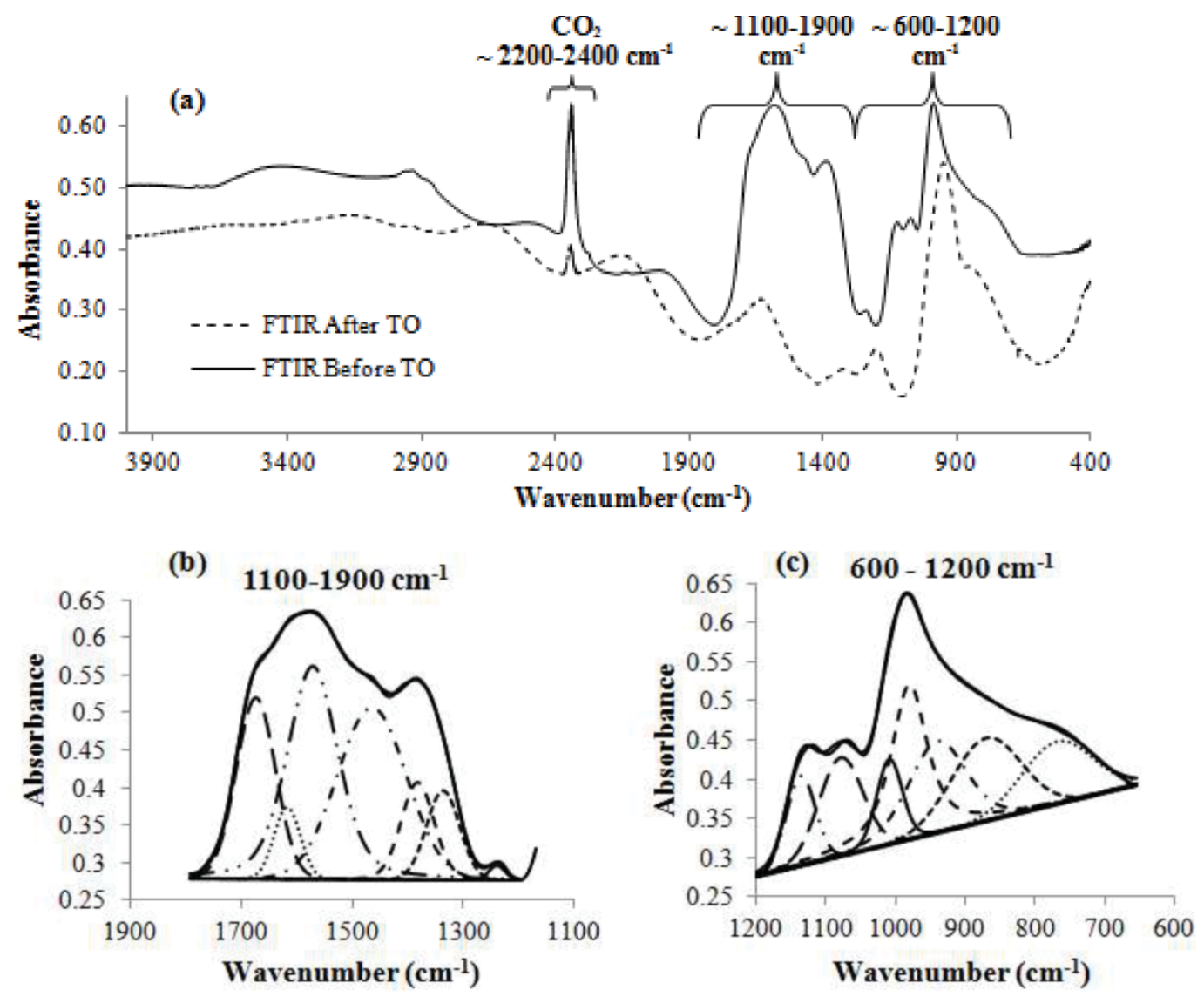

\begin{tabular}{|c|c|c|c|}
\hline & Possible bonds stretch & $\begin{array}{c}\text { Peak position } \\
\left(\mathrm{cm}^{-1}\right)\end{array}$ & Area \\
\hline \multirow{7}{*}{$-\cdots$} & $\mathrm{NH}_{2}$ & 1570.4 & 37.4 \\
\hline & $\mathrm{H}-\mathrm{N}-\mathrm{H}$ & 1672.2 & 21.4 \\
\hline & $\mathrm{NH}_{4}^{-}$ & 1464.1 & 35.8 \\
\hline & Al-O & 1382 & 10.2 \\
\hline & $\mathrm{Al}=\mathrm{O}$ & 1367.4 & 8.9 \\
\hline & Adsorbed $\mathrm{H}_{2} \mathrm{O}$ & 1616 & 5.6 \\
\hline & Absorbed $\mathrm{NH}_{3}$ on $\mathrm{TiO}_{2}$ & 1239.7 & 0.8 \\
\hline
\end{tabular}

\begin{tabular}{|c|c|c|c|}
\hline & Possible bonds stretch & $\begin{array}{c}\text { Peak position } \\
\left(\mathrm{cm}^{-1}\right)\end{array}$ & Area \\
\hline \multirow{7}{*}{$\begin{array}{l}-\cdots \\
-\cdots \\
--- \\
-\cdots- \\
-\cdots\end{array}$} & $\mathrm{Ti}-\mathrm{OH}$ & 1135 & 6 \\
\hline & $\mathrm{Al}-\mathrm{O}-\mathrm{H}$ & 1077.9 & 9.7 \\
\hline & $\mathrm{Al} \cdot \mathrm{OH}$ & 980 & 17.6 \\
\hline & Amorphous Ti-O & 940 & 13.6 \\
\hline & Anatase $\mathrm{TiO}_{2}$ & 870 & 13.2 \\
\hline & Amorphous Ti-O & 768.9 & 10.1 \\
\hline & $\mathrm{Ti}-\mathrm{OH}$ & 1008.4 & 5.2 \\
\hline
\end{tabular}

(d) $1100-1900 \mathrm{~cm}^{-1}$

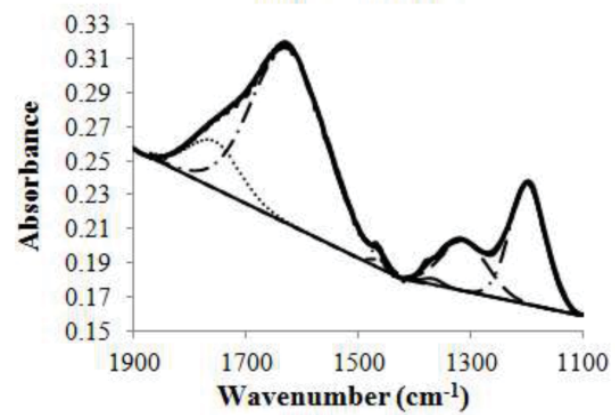

(e)

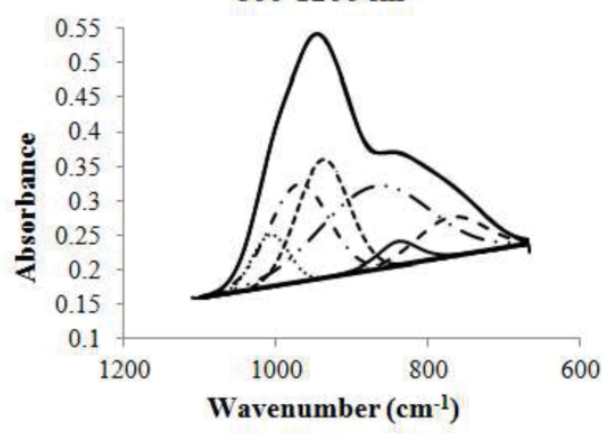

\begin{tabular}{|c|c|c|c|}
\cline { 2 - 4 } \multicolumn{1}{c|}{} & Possible bonds stretch & $\begin{array}{c}\text { Peak position } \\
\left(\mathbf{c m}^{-1}\right)\end{array}$ & Area \\
\hline-- & $\mathrm{Ti}-\mathrm{OH}$ & 1311.5 & 3.2 \\
\hline$-\cdots$ & Adsorbed $\mathrm{NH}_{3}$ on $\mathrm{TiO}_{2}$ & 1198.4 & 5.9 \\
\hline & $\mathrm{Al}=\mathrm{O}$ & 1367.8 & 0.1 \\
\hline$-\cdots$ & $\mathrm{Adsorbed} \mathrm{H}_{2} \mathrm{O}$ & 1624.3 & 17.3 \\
\hline & $\mathrm{NH}_{4}{ }^{+}$ & 1464.8 & 0.1 \\
\hline--- & $\mathrm{NH}_{4}^{+} / \mathrm{CO}_{3}{ }^{2-}$ & 1755.9 & 3.2 \\
\cline { 2 - 4 } & & & \\
\cline { 2 - 4 } & & & \\
\hline
\end{tabular}

\begin{tabular}{|c|c|c|c|}
\cline { 2 - 4 } \multicolumn{1}{c|}{} & Possible bonds stretch & $\begin{array}{c}\text { Peak position } \\
\left(\mathbf{c m}^{-1}\right)\end{array}$ & Area \\
\hline$-\cdots$. & Anatase $\mathrm{TiO}_{2}$ & 870 & 21.5 \\
\hline--- & Amorphous $\mathrm{Ti}-\mathrm{O}$ & 770 & 6.4 \\
\cline { 2 - 4 } & $\mathrm{Al}-\mathrm{OH}$ & 970.4 & 13.7 \\
\hline$\cdots-.$. & Amorphous $\mathrm{Ti}-\mathrm{O}$ & 938.8 & 14.6 \\
\hline$\cdots \cdots . .$. & $\mathrm{Ti}-\mathrm{OH}$ & 1007.9 & 4.5 \\
\cline { 2 - 4 } & $\mathrm{Al}_{2} \mathrm{O}_{3}$ & 840 & 3 \\
\hline
\end{tabular}

84 
Figure 5.4 FTIR spectra of anodized-smooth Ti-6Al-4V samples (a) full spectrum for before (solid line) and after (dashed line) thermal oxidation at $450{ }^{\circ} \mathrm{C}$ for $3 \mathrm{hrs}$ in ambient air; de-convolution of the featured regions (1100-1900 and 600-1200 $\left.\mathrm{cm}^{-1}\right)$ shown in (b, c) before TO and (d, e) after TO. Legend in (b, c, d, and e) provides the possible bonds stretch, peak position, and area under the curves.

Furthermore, the peaks in the $600-1200 \mathrm{~cm}^{-1}$ region before and after TO (Figures $5.4 \mathrm{c}$ and 5.4e) have been assigned to Ti-OH at $1135,1008.4$ and $1007.9 \mathrm{~cm}^{-1}, \mathrm{Al}-\mathrm{OH}$ at 980 and $970.4 \mathrm{~cm}^{-1}, \mathrm{Al}-\mathrm{O}-\mathrm{H}$ at $1077.9 \mathrm{~cm}^{-1}$, amorphous Ti-O at 940, 938.8, 770 and $768.9 \mathrm{~cm}^{-1}$, anatase $\mathrm{TiO}_{2} 870 \mathrm{~cm}^{-1}$ and $\mathrm{Al}_{2} \mathrm{O}_{3}$ at $840 \mathrm{~cm}^{-1}$. [139,144,145,232] Increase in infrared feature intensity for anatase $\mathrm{TiO}_{2}$ at $870 \mathrm{~cm}^{-1}$ and $\mathrm{Al}_{2} \mathrm{O}_{3}$ at $840 \mathrm{~cm}^{-1}$ appears after TO due to the oxidation of Ti-6Al-4V.

Earlier studies have reported that amorphous $\mathrm{TiO}_{2}$ can crystallize into anatase and/or rutile when Ti-6Al-4V samples are thermally oxidized at different temperatures. Anatase $\mathrm{TiO}_{2}$ has been reported to be thermodynamically stable and is composed of facets like (101), (001), and (011); ${ }^{[237-240]}$ moreover, depending on the facet that is exposed, water molecules can dissociatively or non-dissociatively adsorb on the surface. ${ }^{[237-239,241]}$ It has been reported that when the (001) facet of anatase comes in contact with $\mathrm{H}_{2} \mathrm{O}$ molecules, it spontaneously dissociates $\mathrm{H}_{2} \mathrm{O}$ into $\mathrm{H}^{+}$and $\mathrm{OH}^{-}$ions; these ions can attract more $\mathrm{H}_{2} \mathrm{O}$ molecules, thus creating a super-hydrophilic surface after TO. ${ }^{[237-239,241]}$

The FTIRS data reveals a number of important findings: (i) For rough samples, the weak $\mathrm{TiO}_{2}$ peak is observed at $898 \mathrm{~cm}^{-1}$ before TO and at $833 \mathrm{~cm}^{-1}$ after TO. The downshift of the $\mathrm{TiO}_{2}$ peak is likely due to the change in $\mathrm{TiO}_{2}$ structure from amorphous to anatase and/or rutile during the TO treatment. (ii) For smooth (Figure 5.3) and anodized-smooth (Figure 5.4) samples, an increase of the $\mathrm{TiO}_{2}$ peak intensity is observed after TO; this increase is attributed to the increased amount of $\mathrm{TiO}_{2}$ present after TO. (iii) For anodized samples (Figures 5.4), the intensities of peaks assigned to $-\mathrm{NH}_{2}, \mathrm{H}-\mathrm{N}-\mathrm{H}$, $\mathrm{NH}_{4} \mathrm{~F}$ and absorbed $\mathrm{NH}_{3}$ on $\mathrm{TiO}_{2}$ bond stretching are seen to decrease after TO; these species are from the anodization electrolyte and may have evaporated or desorbed during TO. Finally, (iv) for the anodized samples the peak intensities of anatase $\mathrm{TiO}_{2}$ and $\mathrm{Al}_{2} \mathrm{O}_{3}$ 
are found to increase after TO (Figures 5.4c and 5.4e). FTIRS data in this study are in agreement with those reported by D. H. Shin et al., although detailed analysis and discussion of the FTIRS features were lacking in that study. ${ }^{[218]}$

The de-convolution may include peak position deviation of the absorption bands from literature values due to 1) uncertainities from the sloped baseline due to irregular surface roughness, and 2) absorption shift (blue shift or red shift) for particular bond stretch due to the matrix effect of different chemical species within the vicinity. Consequently, further study with controlled parameters is required to optimize the deconvolution of FTIRS data.

GIXRD spectrum of thermally oxidized Ti-6Al-4V samples at $450{ }^{\circ} \mathrm{C}$ for 3 hours in ambient environment is shown in Figure 5.5. It is observed that i) intensity at 2-theta of 35.094 and 62.950 for $\mathrm{Ti}$ in control decreases after heat-treatment at $450{ }^{\circ} \mathrm{C}$, ii) simultaneously, it is also observed that peaks at 2-theta of 38.422 and 53.005 found in control broadened and increased in intensity after heat-treatment, which may indicate the presence of anatase features at $37.790\left(\begin{array}{lll}0 & 0 & 4\end{array}\right)$ and $53.885\left(\begin{array}{lll}1 & 0 & 5\end{array}\right)$ 2-theta as anatase peaks are observed in close proximity with $\mathrm{Ti}$ peaks at that region, and iii) no anatase features appear at 2-theta of 25.425 (llll 101 ) possibly due to the preferred grain orientation of the anatase crystal for the thin films, where $\left(\begin{array}{lll}1 & 0 & 1\end{array}\right)$ facet for anatase may not be oriented in such a way that it could be detected by GIXRD. No rutile features appeared in the GIXRD data, which may be due to insufficient amount of its presence in the thin film. Previously, it is observed that when Ti-6Al-4V samples are heat treated at $600{ }^{\circ} \mathrm{C}$ for 1 hour, no features for rutile appeared ${ }^{[242]}$, however, in this study the samples were heattreated at same temperature for 3 hours and rutile peaks appeared. This suggests that though rutile may be present when samples are annealed at $450{ }^{\circ} \mathrm{C}$ for 3 hour, it may be below GIXRD detection limit. 


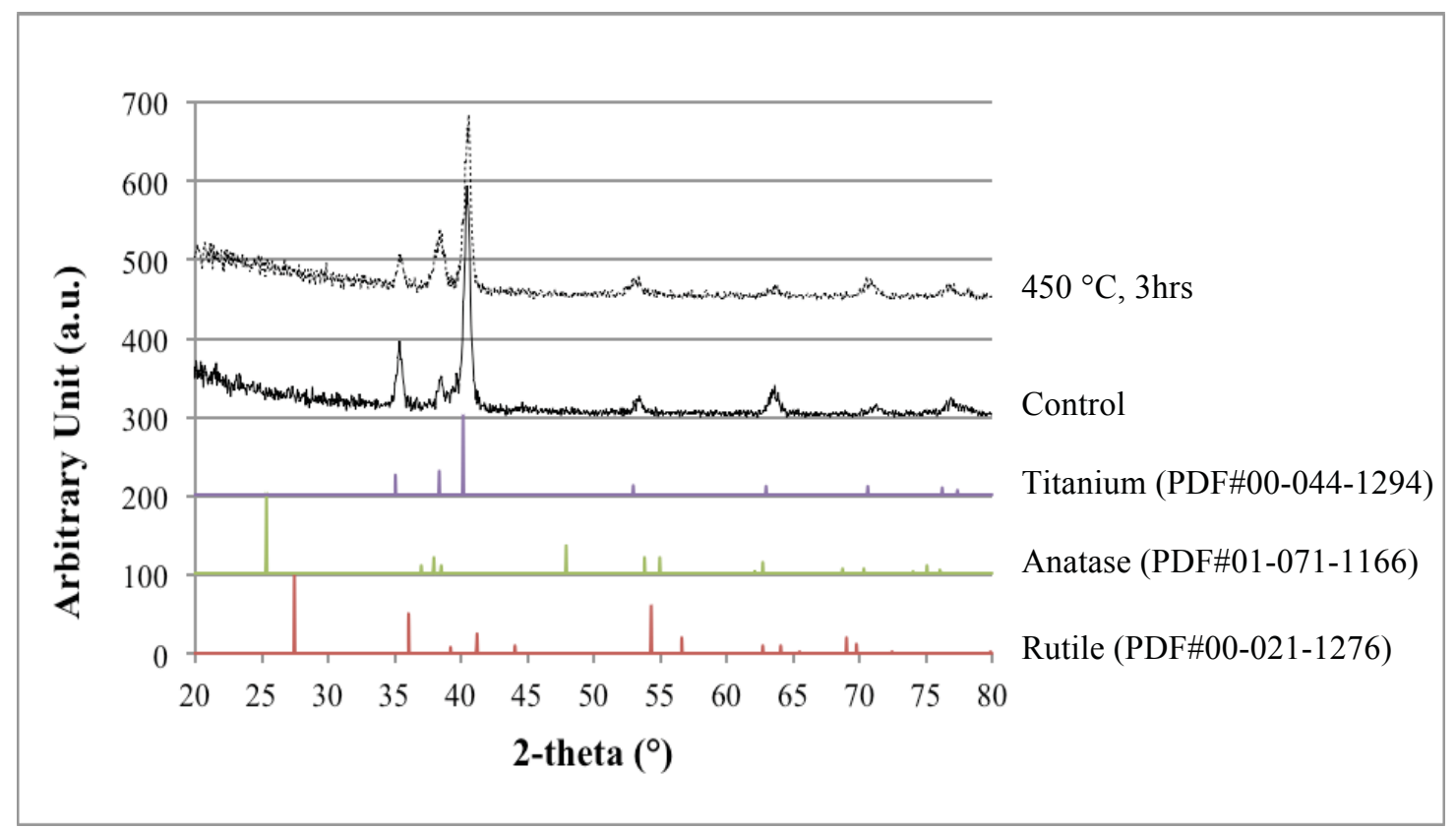

Figure 5.5 GIXRD of control (solid line) and annealed (dashed line) Ti-6Al-4V at $450{ }^{\circ} \mathrm{C}$ for 3 hours in to the furnace in ambient environment. XRD baselines for titanium, anatase $\mathrm{TiO}_{2}$ and rutile $\mathrm{TiO}_{2}$ is also provided.

\section{Surface wettability}

WCA measurements of smooth, anodized-smooth, rough and anodized-rough samples at various stages of the experiments are studied next (Table 5.1). Figure 5.6 shows the WCA results before and after thermal oxidation of the Ti-6Al-4V substrates. The WCA of as prepared samples (step 1) are $57 \pm 6^{\circ}$ for smooth, $86 \pm 7^{\circ}$ for rough, and less than $5^{\circ}$ for anodized-smooth and anodized-rough samples. 


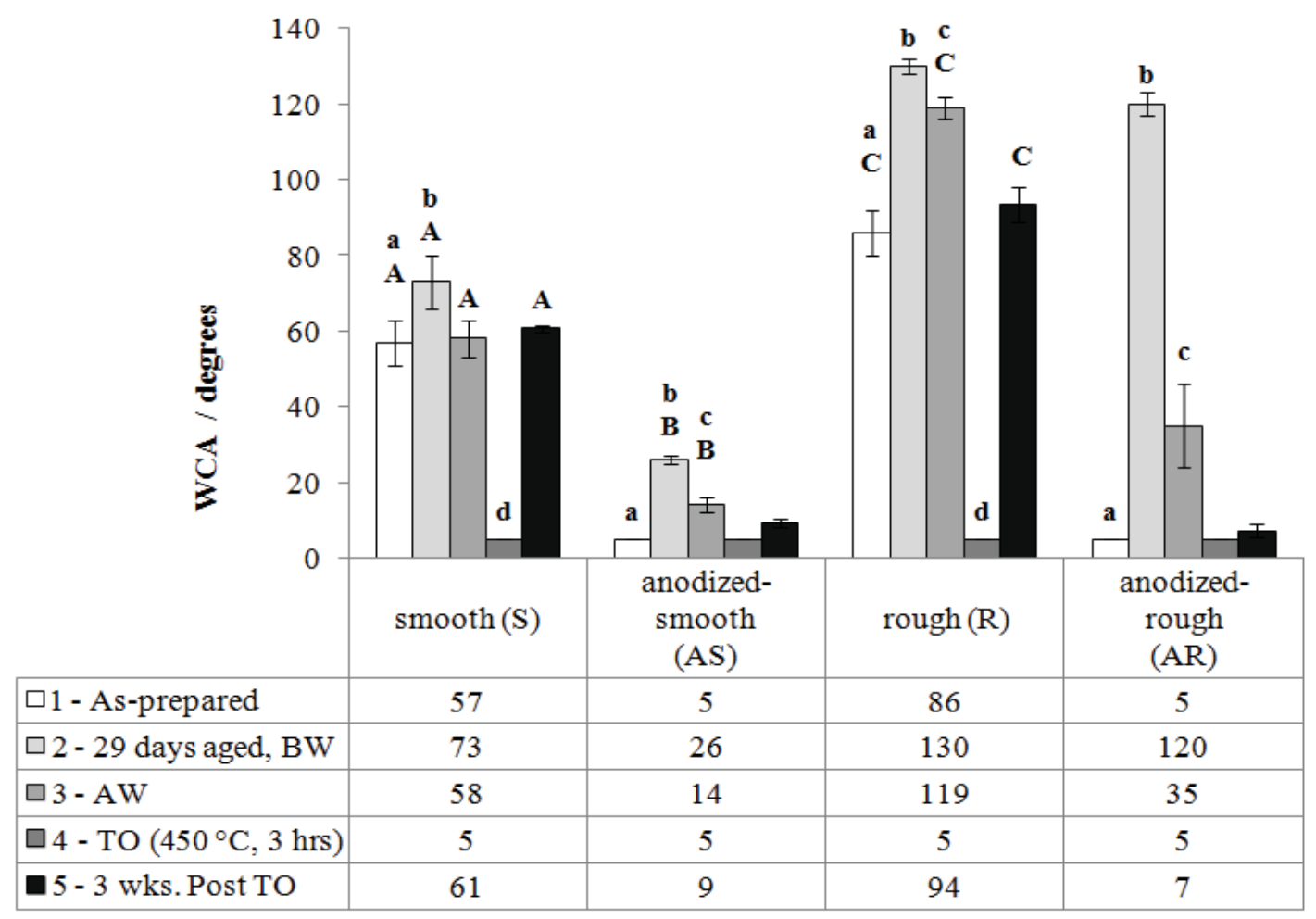

Figure 5.6 Comparison of surface wettability of samples with different surface topographies at various stages of experiment. Average WCA is recorded in degrees. BW $=$ Before DI-water Wash, $\mathrm{AW}=$ After DI-water Wash, TO = Thermal Oxidation, 3 wks. $=3$ Weeks. Samples were wrapped in kimwipes and stored in sealed centrifuge plastic vials during aging period. a: $p<0.0001$ compared to step 2; b: $p<0.0001$ compared to steps 3, 4 and 5; c: $p<0.0001$ compared to steps 4 and 5; $\mathrm{d}: p<0.0001$ compared to step 5; A: $p<0.0001$ compared to groups AS, $\mathrm{R}$ and AR. B: $p<0.0001$ compared to group $\mathrm{R}$ and AR; $\mathrm{C}: p<0.0001$ compared to group AR

As-prepared Ti-6Al-4V rough samples show hydrophobic behavior likely due to residuals from the acid etching solution, alumina particles from sandblasting, or air entrapment beneath the water droplet due to micron-level rough surface. Earlier studies have focused on understanding the wettability behavior of rough samples using two models, Wenzel and Cassie Baxter (CB). The Wenzel model is used when the surface 
composition is homogenous and air entrapment inside the micro-grooves is not considered, whereas the $\mathrm{CB}$ model is used when the surface composition is heterogeneous and air entrapment inside the micro-grooves is considered. ${ }^{[243,244]}$ Since Ti-6Al-4V surface is heterogeneous, WCA data in this study can be analyzed using the CB theory.

There are two parameters that affect surface hydrophilicity: surface free energy (SFE) and surface roughness. ${ }^{[245,246]}$ Different elements possess different SFE; therefore, when a WCA measurement is performed on an alloy, the tendency of the water to bond depends on the SFE of the elements on the surface which makes contact with the water. The higher the SFE of the elements in contact with water, the more that surface region will be covered with water. ${ }^{[245-247]}$ In this study, the high WCA of $86 \pm 7^{\circ}$ on as-prepared rough surface could be due to the high free energy barrier present on this alloy consisting

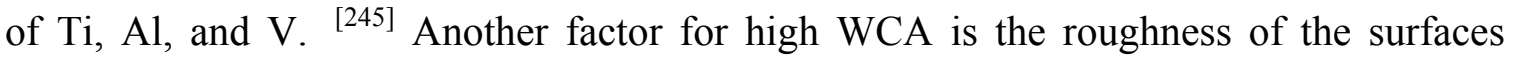
(smooth vs. rough). ${ }^{[248,249]}$ If the WCA of a rough heterogeneous surface is greater than $80-90^{\circ}$, then the surface is hydrophobic due to the lack of capillary channeling effect which results into air entrapment inside the micro-gooves on the surface; ${ }^{[250]}$ this can further prevent water from forming hydroxyl bonds with the susbtrate. ${ }^{[247-249,251]}$

The effect of surface roughness on wettability has been investigated by Oliver $e t$ al. on aluminum substrates using ethylene glycol as the wetting agent. The study shows that as the surface roughness increased from Ra less than $0.5 \mu \mathrm{m}$ (polished surface) to Ra $=2.4 \mu \mathrm{m}$ (spiral: coarse topography), the WCA slightly increased from $61 \pm 1.9^{\circ}$ to 69.8 $\pm 2^{\circ}$. Additionally, a similar wettability trend was observed for stainless steel samples using ethylene glycol; that is, the contact angle on a polished surface was $60.2 \pm 1.6^{\circ}$, while on a rough surface was $106.7 \pm 3.6^{\circ} .{ }^{[252]}$ This suggests that, when ethylene glycol contact angles were probed on aluminum and stainless steel, similar contact angle results were obtained for smooth samples; this may be due to surfaces with similar SFE; however, when different morphologies of roughness were introduced on aluminum (spiral:coarse topography) and stainless steel (orthogonal array of holes) substrates, the resulting contact angles differed significantly. This WCA difference may be due to the higher probability of air entrapment inside the orthogonal array holes (stainless steel 
surface roughness) than in a spiral coarse topography (aluminum). In this study, a similar trend of increasing WCA with increasing surface roughness is observed that is WCA of $57 \pm 6^{\circ}$ for smooth and $86 \pm 7^{\circ}$ for rough Ti-6Al-4V surfaces.

Studies by Rupp et al. and Le Guehennec et al. reported the embedment of $\mathrm{Al}_{2} \mathrm{O}_{3}$ particles as well as excess carbon contamination on the metal surface after sandblasting as observed by XPS analysis leading to a hydrophobic surface for rough substrates. ${ }^{[221,251,253]}$ However, it is not clear if the increased WCA of sandblasted and acid etched samples is due to surface roughness or higher chemical contamination than on smooth samples.

On the other hand, as-prepared anodized samples show super-hydrophilic behavior with a WCA of less than $5^{\circ}$ (Figure 5.6) in qualitative agreement with results reported by Shin et al. at similar experimental conditions. In that study, Ti-6Al-4V substrates were anodized in ammonium fluoride and ethylene glycol solution and they exhibited a weak hydrophilic behavior with a WCA of $32^{\circ}$, in contrast with the super hydrophilicity of the substrates in this study with the WCA of less than $5^{\circ}$; on the other hand, Shin et al. reported the WCA of non-anodized smooth Ti-6Al-4V surfaces to be $67^{\circ}$, which is close to the one observed in this experiments (Figure 5.6). ${ }^{[218]}$ The difference in the wettability characteristics of the anodized samples may be due to the different impurity levels. The hydrophilic behavior of anodized samples in fluorinecontaining electrolytes is likely due to the formation of TNTs with $\operatorname{Ti}(\mathrm{OH})_{4}$ surface composition. ${ }^{[218]}$

When ethylene glycol is used along with ammonium fluoride, nanotubes consisting of $\mathrm{Ti}(\mathrm{OH})_{4}$ are obtained. ${ }^{[218]}$ If ammonium fluoride solution is used with other type of solutions such as $\left(\mathrm{NH}_{4}\right)_{2} \mathrm{SO}_{4},{ }^{[222]}$ fluoride-containing nanotubes may be obtained with likely $\mathrm{TiF}_{4}$ surface composition as opposed to $\mathrm{Ti}(\mathrm{OH})_{4}$. Further, as it was indicated in the FTIRS section above, hydroxyl groups on the TNT walls may attract $\mathrm{H}_{2} \mathrm{O}$ molecules that make the anodized surface hydrophilic. The behavior of anodized Ti-6Al$4 \mathrm{~V}$ substrates has been reported previously; however, their electrolyte composition, counter electrode, applied voltage and anodization duration differ from those of our 
study; ${ }^{[101,222,223,254-261]}$ Moreover, the chemical composition of nanotube walls depends on the electrolyte in which the nanotubes are etched.

The WCA of samples after 29 days of aging without a DI-water wash (step 2, Table 5.1 and Figure 5.6) are found to increase to $73 \pm 8^{\circ}$ for smooth, $26 \pm 1^{\circ}$ for anodized-smooth, $130 \pm 2^{\circ}$ for rough, and $120 \pm 3^{\circ}$ for anodized-rough surfaces. The observed increase in WCA after aging of the surfaces may have been caused by the surface contamination from organic and/or inorganic impurities in the environment. After cleaning the samples with a DI-water wash (step 3, Table 5.1 and Figure 5.6), the WCA are found to decrease to $58 \pm 6^{\circ}$ for smooth, $14 \pm 2^{\circ}$ for anodized-smooth, $119 \pm 3^{\circ}$ for rough and $35 \pm 12^{\circ}$ for anodized-rough samples. Though there is a decrease in the WCA on all samples after the DI-water wash, the surface wettability of smooth and rough samples apparently remained consistently high.

Immediately after thermal oxidation at $450{ }^{\circ} \mathrm{C}$ for 3 hours (step 4, Table 5.1 and Figure 5.6), the WCA for all sample decreased to less than $5^{\circ}$. That is, a significant decrease in the WCA of rough and smooth samples is observed after thermal oxidation when compared to WCA of as-prepared samples ( $p<0.0001$; Tukey HSD post hoc analysis). This change of surface wettability from hydrophobic to hydrophilic for smooth and rough surfaces after TO is probably due to the formation of anatase structure $\mathrm{TiO}_{2}$ (also see the previous FTIRS section). However, anodized-smooth and anodized-rough samples maintain their hydrophilic property due to following reasons: i) after TO, the walls of nanotubes were crystallized to anatase and rutile, which has the ability to split water into $\mathrm{H}^{+}$and $\mathrm{OH}^{-}$ions, thereby forming hydroxyl groups on the surface that can attract the water molecule making surface hydrophilic, and 2) due to possessing the tubular morphology, it has the capillary effect that further draws the water towards the sample making the surface even more hydrophilic.[262]

Finally, three weeks after TO (step 5, Table 5.1 and Figure 5.6), the WCA of smooth and rough samples were found to have increased to almost their initial wettability levels of $61 \pm 1^{\circ}$ and $94 \pm 5^{\circ}$, respectively. Such increase in WCA is likely due to the loss of $\mathrm{OH}^{-}$groups from the surface. Hydroxyl groups attract the water molecules during 
WCA, thereby making surface hydrophilic. On the other hand, the WCA of the anodizedrough and anodized-smooth samples remained below $10^{\circ}$ after the same period of time. This may be due to the retention of $\mathrm{OH}^{-}$group by anatase and rutile inside the tubular cavities of nanotubes for a longer time compared to non-anodized samples. Anodized samples have a higher surface area compared to non-anodized samples, and therefore they have higher amount of $\mathrm{OH}^{-}$groups on the surface. This suggests that after aging, $\mathrm{OH}^{-}$groups from the non-anodized samples may have been dissociated completely; however, $\mathrm{OH}^{-}$group from anodized samples might have been dissociated partially maintaining surface hydrophilicity over-time. The aging effect on the surface wettability before and after TO provides essential information regarding the rate at which the wettability characteristics of samples change because of ambient contamination, chemical changes on the sample surfaces, storage conditions, etc. In this work, thermal oxidation of anodized samples at the conditions investigated effectively maintains the hydrophilicity of Ti-6Al-4V surfaces for a long time.

\subsection{Experimental}

\section{Sample Preparation}

For a rough surface, $15 \mathrm{~mm}$ in diameter and $1 \mathrm{~mm}$ thick Ti-6Al-4V alloy discs (McMaster-Carr, Elmhurst IL, USA) were sandblasted by vertically spraying $50 \mu \mathrm{m}$ diameter alumina $\left(\mathrm{Al}_{2} \mathrm{O}_{3}\right)$ particles (Trinity Tool Company, Fraser MI, USA) at $517 \mathrm{kPa}$ for $10 \mathrm{~s}$. Then the samples were acid-etched in sulfuric acid and $30 \%$ hydrogen peroxide (solution mixture, volume ratio 1:1) for two hours. For a smooth surface, a standard metallographic preparation protocol was used. Ti-6Al-4V discs were wet-grounded using a series of silicon carbide grinding papers \#320, \#400, \#600, and \#800 (Carbimet 2, Buehler, Lake Bluff, IL, USA), followed by a polishing cloth (TexMet Polishing Cloth, Beuhler, Lake Bluff, IL, USA) with diamond paste (MetaDi 9-micron, Beuhler, Lake Bluff, IL, USA) and lubricant (MetaDi Fluid, Beuhler, Lake Bluff, IL, USA). Chemomet polishing cloth (Chemomet I, Buheler, Lake Bluff, IL, USA) with colloidal silica 
polishing suspension (MasterMed, Buehler, Lake Bluff, IL, USA) was used as a final polishing procedure in order to obtain a mirror-like surface roughness.

\section{Procedure for Anodization}

Rough and smooth samples were anodized in order to obtain nanotubes on their surfaces. Each Ti-6Al-4V sample was used in an electrochemical cell as the working electrode, while a copper rod was used as the counter electrode. Both electrodes were connected to a voltage source (Keithley 2400 SourceMeter, Cleveland, OH, USA) and immersed in an electrolyte solution composed of ethylene glycol (Fisher Scientific, Pittsburgh, PA, USA), 0.3 wt. \% $\mathrm{NH}_{4} \mathrm{~F}$ (Sigma-Aldrich, St. Louis, MO, USA) and DI water $(2$ vol. \%). A voltage of $60 \mathrm{~V}$ was maintained for four hours and the electrolyte solution was continuously stirred to maintain a homogenous environment.

\section{Procedure for thermal oxidation}

Thermal oxidations were carried out in a furnace (One zone blue lindberg furnace, $54032,120 / 240$ Volts, maximum temperature of $\left.1200^{\circ} \mathrm{C}, 50 / 60 \mathrm{~Hz}, 960 \mathrm{~W}\right)$. All samples were thermally oxidized at $450{ }^{\circ} \mathrm{C}$ for three hours in ambient air. A temperature controller (JLD-612 temperature controller, Sacramento, CA, USA) was used to maintain the working temperature of $450{ }^{\circ} \mathrm{C}$. Prior to oxidation, the samples were cleaned with DIwater of $17.7 \mathrm{M} \Omega-\mathrm{cm}$ resistivity (Barnstead NANOPure, Infinity Ultrapure Water System ${ }^{\circledR}$ ) followed by $\mathrm{N}_{2}$ gas drying (Grade 4.8, 99.998\%, NIZ300 Progressive Industries Inc., Sylacauga, Al, USA). After thermal oxidation, the samples were slowcooled by pulling them out of the furnace tube approximately $5 \mathrm{~cm}$ every 7 minutes. This prevents/minimizes the production of micro-cracks caused by abrupt temperature changes on the sample surface.

Field Emission Scanning Electron Microscopy (FE-SEM) 
FE-SEM (JSM-6320F, JEOL, Musashino 3-chome Akishima Tokyo, Japan) was used to analyze the surface morphology of smooth, rough, anodized-smooth and anodized-rough Ti-6Al-4V samples before and after TO. The sample of interest was mounted on an aluminum stub with double sided conductive carbon tape for imaging. FESEM images were obtained with acceleration voltages of 3, 4, 5, 9 and $10 \mathrm{kV}$, and specimen magnifications of 10,000 X and 55,000 X.

\section{White-light interferometry}

White-light interferometric microscope (NewView 6300, Zygo Corporation, Middlefield, Connecticut, USA) was used to measure surface roughness and generate a 3D profile of each surface. White-light interferometry was performed on all samples before and after thermal oxidation in order to probe related changes in the surface roughness.

\section{Water contact angle (WCA) measurements}

A sessile drop method was used to perform WCA measurements (CA Goniometer, Rame'-Hart NRL, Succasunna, NJ, USA). A micro-syringe (Hamilton, Reno, NV, USA) was used to place a $5 \mu \mathrm{L}$ DI-water droplet onto anodized and nonanodized smooth and rough Ti-6Al-4V surfaces. The wettability characteristics of the samples were monitored with WCA measurements at various stages of the experiments: 1) as-prepared, 2) 29 days after their receipt and before DI-water wash (BW), 3) after DIwater wash (AW), 4) immediately after heat treatment and 5) thee weeks after heat treatment. Prior to the WCA measurements for step 1, the samples were cleaned with DIwater for one minute followed by $\mathrm{N}_{2}$ gas drying. After all WCA measurements, the samples were wrapped in kimwipes (Kimtech Science, Kimberly-Clark Corporation, Roswell, GA, USA) in conical bottom centrifuge tubes (Corning, sterile, $50 \mathrm{ml}$ clear polypropylene/PP, Tewksbury, MA, USA). 


\section{Fourier Transform Infrared Spectroscopy (FTIRS)}

The surface composition of smooth and anodized-smooth Ti-6Al-4V samples before and after TO were studied using diffuse reflectance FTIRS (FTIR, Nicolet, Madison, WI, USA). Smooth samples were used as a control/background. Prior to conducting FTIRS analyses, each sample was kept inside the FTIRS chamber during purging for one hour in order to optimize signal to noise ratio. FTIRS spectra were obtained with $1 \mathrm{~cm}^{-1}$ resolution and 512 scans over the $4000-400 \mathrm{~cm}^{-1}$ range. Qualitative and semi-quantitative analysis of FTIR spectra was performed using spectral peak-fitting software. FTIRS peaks were analyzed with linear background and deconvoluted using Gaussian-Lorentian peak shape function.

Grazing incidence X-ray diffraction (GIXRD)

Crystallinity of Ti-6Al-4V samples subjected to TO was measured using high resolution GIXRD (Philips X'pert 2) configured with the $0.1542 \mathrm{~nm}$ x-ray emission line of $\mathrm{Cu}$. The GIXRD spectrum was collected at the angle of incidence of $1^{\circ}$ to increase the sensitivity of crystalline $\mathrm{TiO}_{2}$ thin film.

\section{Statistical Analysis}

One-way ANOVA was used to determine the differences in surface wettability, average surface roughness and root mean square roughness of various surface topographies of Ti-6Al-4V samples. Tukey HSD post hoc analysis was used for pair-wise comparisons within these groups. Statistical software (SPSS v. 20.0, SPSS Inc., Chicago, IL, USA) was used for descriptive and statistical analyses. For all analyses, p-values $<0.05$ was considered statistically significant. 


\subsection{Conclusions}

Smooth, anodized-smooth, rough and anodized-rough Ti-6Al-4V surface characteristics before and after thermal oxidation in ambient environment were studied. Anodized Ti-6Al-4V samples were found to be super-hydrophilic before TO, after TO and post-TO aging with WCA less than $10^{\circ}$ likely due to the retention of hydroxyl groups inside the nanotubes for a longer period of time compared to non-anodized samples.

White-light interferometry analysis indicated a slight increase in surface roughness of smooth samples due to $\mathrm{TiO}_{2}$ crystallization from amorphous to anatase and rutile; however, the surface roughness for anodized samples is found to decrease after TO likely due to detachment and accumulation of nanotubes on the surface.

FTIRS analysis of thermally oxidized samples at $450{ }^{\circ} \mathrm{C}$ showed the presence of anatase and rutile crystalline $\mathrm{TiO}_{2}$ structure, which explains i) hydrophilic behavior of all samples after TO, ii) increase in surface roughness of smooth samples after TO, and iii) decrease in surface roughness after TO. GIXRD analysis corroborated the FTIRS analysis in regards to the presence of anatase $\mathrm{TiO}_{2}$.

Studying surface wettability, chemical composition, external morphology and surface roughness of non-anodized and anodized smooth and rough surfaces before and after TO and understanding the behavior of the samples with different surface roughness at various stages of the experiments is essential to correlate cellular response with the samples from each one of these groups. Improved osseointegration is one of the key factors for prolonged success of dental and orthopedic implants. While an anodized surface may result in improved cellular response, one of its limitations may include detachment of nanotubes from the substrate due to the micro-motions of physiological activity. Therefore, to understand the relationship between tribology and cellular response, a study including tribological along with cellular responses would be helpful. 


\section{Acknowledgements}

The authors would like to thank Lukasz Zientara for the furnace set-up, Jorge Iván Rossero A. for his valuable assistance in spectral peak fitting software and GIXRD analysis. Sathees Selvaraj and Dr. Mauro Sardela from Frederick Seitz Materials Research Laboratory Central Facilities from University of Illinois at Urbana-Champaign for their help and support in obtaining GIXRD data. Partial financial support by the Department of Bioengineering at UIC is gratefully acknowledged by the lead author. Partial financial support by the National Science Foundation (CBET \# 1067424 and CMS \# 0609377) is gratefully acknowledged. 


\section{Chapter 6}

\section{A Novel Investigation of the Formation of TiO2 Nanotubes on Thermally Formed Oxide of Ti-6Al-4V*}

Arman Butt ${ }^{1}$, Azhang Hamlekhan ${ }^{2}$, Sweetu Patel ${ }^{2}$, Dmitry Royhman ${ }^{3}$, Cortino Sukotjo ${ }^{3}$, Mathew T. Mathew ${ }^{4}$, Tolou Shokuhfar ${ }^{2,5,6}$, Christos Takoudis ${ }^{1,7,8}$

${ }^{1}$ Department of Bioengineering, University of Illinois at Chicago, Chicago, IL 60607, USA

${ }^{2}$ Mechanical Engineering-Engineering Mechanics, Michigan Technological University, Houghton, MI 49931, USA

${ }^{3}$ Department of Restorative Dentistry, College of Dentistry, University of Illinois at Chicago, Chicago, IL 60612, USA

${ }^{4}$ Department of Orthopedics, Rush University Medical Center, Chicago, Chicago, IL 60612, USA

${ }^{5}$ Department of Mechanical and Industrial Engineering, University of Illinois at Chicago, Chicago, IL 60607, USA

${ }^{6}$ Department of Physics, University of Illinois at Chicago, Chicago, IL 60607, USA

${ }^{7}$ Department of Chemical Engineering, University of Illinois at Chicago, Chicago, IL 60607, USA

${ }^{8}$ Corresponding author

*The material contained in this chapter has been published in the Journal of Oral Implantology. My contribution to this publication includes fabrication of nanotubes, FESEM, EDX and reviewing the manuscript. 


\begin{abstract}
Traditionally, $\mathrm{TiO}_{2}$ nanotubes (TNTs) are anodized on Ti-6Al-4V alloy (Ti-V) surfaces with native titanium oxide (amorphous $\mathrm{TiO}_{2}$ ); subsequent heat treatment of anodized surfaces has been observed to enhance cellular response. As-is bulk Ti-V, however, is often subjected to heat treatment, such as thermal oxidation (TO), to improve its mechanical properties. TO-treatment of Ti-V above 200 and $400{ }^{\circ} \mathrm{C}$ initiates the formation of anatase and rutile $\mathrm{TiO}_{2}$, respectively, which can affect TNT formation. This study aims at understanding the TNT formation mechanism on Ti-V surfaces with TOformed $\mathrm{TiO}_{2}$ compared to that on as-is $\mathrm{Ti}-\mathrm{V}$ surfaces with native oxide. TO-formed $\mathrm{TiO}_{2}$ cany affect TNT formation and surface wettability due to the TO-formed $\mathrm{TiO}_{2}$ expected to be part of the TNT structure. Surface characterization was carried out with Field Emission Scanning Electron Microscopy, Energy Dispersive X-Ray Spectroscopy, Water Contact Angle measurements, and White Light Interferometry. TNTs were formed on control, 300 and $600{ }^{\circ} \mathrm{C}$ TO-treated Ti-V samples and significant differences in TNT lengths and surface morphology were observed. No difference in elemental composition was found. TO and TO/anodization treatments produced hydrophilic surfaces, while hydrophobic behavior was observed over time (aging) for all samples. Reduced hydrophobic behavior was observed for TO/anodized samples when compared to control, control/anodized, and TO-treated ones. A method for improved surface wettability and TNT morphology is therefore discussed for possible applications in effective osseointegration of dental and orthopaedic implants.
\end{abstract}

Key words: Thermal Oxidation, Anodization, Anatase, Rutile, Osseointegration, Ti-6Al$4 \mathrm{~V}$ alloy 


\subsection{Introduction}

Ti-6Al-4V alloy (Ti-V) is favored for orthopaedic and dental implants due to superior biocompatibility, density, mechanical resistance, corrosion resistance, atoxicity, chemical inertia, and osseointegration.[6,175,263-268] Specifically, osseointegration is a strong indicator of implant success which can be influenced by surface composition, energy, and topography.[179,183,184,263,266,267,269-275] Surface $\mathrm{TiO}_{2}$ has been shown to improve osseointegration by means of enhanced bone morphogenetic protein (BMP) signaling, apatite formation, up-regulation of collagen II, osteocalcin, biglycan, collagen I, osteopontin and TGF- $\beta 1 .[56,271,273,276-281] \mathrm{TiO}_{2}$ is also known to readily react with water to form hydroxyl groups that increase surface energy and promote cellular attachment.[272-275,282] In addition, the native $\mathrm{TiO}_{2}$ layer prevents dissolution of bulk Ti-V components, aluminum ( $\mathrm{Al}$ ), vanadium (V), and titanium (Ti), into the biological environment (corrosion resistance). $[6,283] \mathrm{Al}$ ions promote scar tissue, $\mathrm{V}$ ions prevent cell division, and $\mathrm{Ti}$ ions promote osteolytic cytokines which can lead to implant loosening; $\mathrm{Al}$ and $\mathrm{V}$ are also mutagenic, cytotoxic and allergenic. [6,283]

The aforesaid surface properties can be improved by enhancing the surface native titanium oxide $\left(\mathrm{TiO}_{2}\right)$ using atomic layer deposition (ALD), chemical vapor deposition (CVD), and thermal oxidation (TO), sandblasting, acid-etching, and anodization.[6,56,139,265,266,282,284-291] ALD is a cyclic self-limiting deposition technique that is highly conformal to surface topography; using tetrakis(diethylamino)titanium (TDEAT) precursor and $\mathrm{H}_{2} \mathrm{O}$ as oxidant, a growth rate of $.5 \AA / m i n$ of $\mathrm{TiO}_{2}$ can be achieved.[193,292-294] Compared to ALD, CVD is a one-step mechanism and using TDEAT precursor and $\mathrm{NH}_{3}$ as catalyst and co-reactant results in growth rate of up to $10 \mathrm{~nm} / \mathrm{min}$ of $\mathrm{TiO}_{2}$.[295] Alternatively, TO-treatment can yield growth rates of $\mathrm{TiO}_{2}$ similar to CVD without the requirement of a precursor or vacuum condition and is governed by the diffusion of oxygen into bulk Ti-V.[296-299] Oxygen from air readily reacts with $\mathrm{Ti}-\mathrm{V}$ at room temperature to form an oxide layer (native oxide) 2-8 $\mathrm{nm}$ thick and $\mathrm{TiO}_{2}$ layer $10 \mathrm{~s}$ of micrometer $(\mu \mathrm{m})$ thick can be achieved within hours with TO-treatment at temperatures of up to $1200{ }^{\circ} \mathrm{C}$.[139,202,268,283,299-301] Additionally, anatase and rutile crystalline structures of $\mathrm{TiO}_{2}$ formed above 200 and 400 
${ }^{\circ} \mathrm{C}$, respectively, have shown to promote basic $\mathrm{OH}^{-}$groups in biological environment $\left(\mathrm{pH} \geq 6.2\right.$ ); basic $\mathrm{OH}^{-}$groups enhance cellular response as compared to acidic $\mathrm{OH}^{-}$ groups.[282,302,303]

$\mathrm{TiO}_{2}$ nanotubes (TNTs) increase surface area on a cellular scale, enhancing human mesenchymal stem cell (hMSC) adhesion.[6,56,174] TNTs are created by an anodic oxidation process using an electrochemical cell and an electrolyte.[6,10,79,81,304-307] TNTs grow as a result of oxidation and dissolution kinetics of $\mathrm{TiO}_{2} \cdot[6,306,307]$ For a fluorine (F) containing electrolyte, the four key processes are i) formation of a fluoride rich layer at the oxide metal interface due to ion migration, ii) displacement of this layer towards the cell boundaries due to a flow mechanism, iii) dissolution of the fluoride rich cell boundaries, iv) and formation of separated TNTs as a result of dehydration.[6,307] TNT aspect ratio (diameter/length) is affected by the applied voltage, fluorine wt. \% electrolyte composition, and anodization duration. [6,167,308] Additionally, thermal treatment of TNTs has shown to provide a super-hydrophilic surface which improves cellular response.[31,79,282] Traditional anodization studies investigate TNTs formed on Ti-V with an amorphous $\mathrm{TiO}_{2}$ surface. $\mathrm{Ti}-\mathrm{V}$, however, is often subject to TO-treatment to improve mechanical properties. TOtreatment initiates anatase and rutile structured $\mathrm{TiO}_{2}$ above 200 and $400{ }^{\circ} \mathrm{C}$, respectively, which may affect TNT formation. This study aims to understand TNT formation mechanism on TO-treated Ti-V with crystalline surface $\mathrm{TiO}_{2}$ compared to that on nontreated $\mathrm{Ti}-\mathrm{V}$ with amorphous surface $\mathrm{TiO}_{2}$.

\subsection{Materials \& Methods}

\section{Sample Preparation}

$12 \mathrm{Ti}-\mathrm{V}$ disks (diameter $=15 \mathrm{~mm}$, height $=1 \mathrm{~mm}$ ) were cut and polished from Ti-V rods (McMaster-Carr). A smooth surface $\left(\mathrm{R}_{\mathrm{a}}=10 \pm 2 \mathrm{~nm}\right)$ was achieved by i) wetgrinding using a series of Carbimet 2 silicon carbide grinding papers (\#200, 320, 400, 600, and 800: Buehler), ii) polished using a TexMet Polishing Cloth (Buehler), MetaDi 9micron diamond paste (Buehler), and MetaDi Fluid lubricant (Beuhler), and iii) finepolished using a Chemomet I polishing cloth (Buheler) with MasterMed colloidal silica 
polishing suspension (Buehler). Samples were washed with DI-water (17.7 M $\Omega$-cm, Barnstead NANOPure) followed by drying with $\mathrm{N}_{2}$ gas spray (Grade 4.8, 99.998\%, Progressive Industries, Inc.).

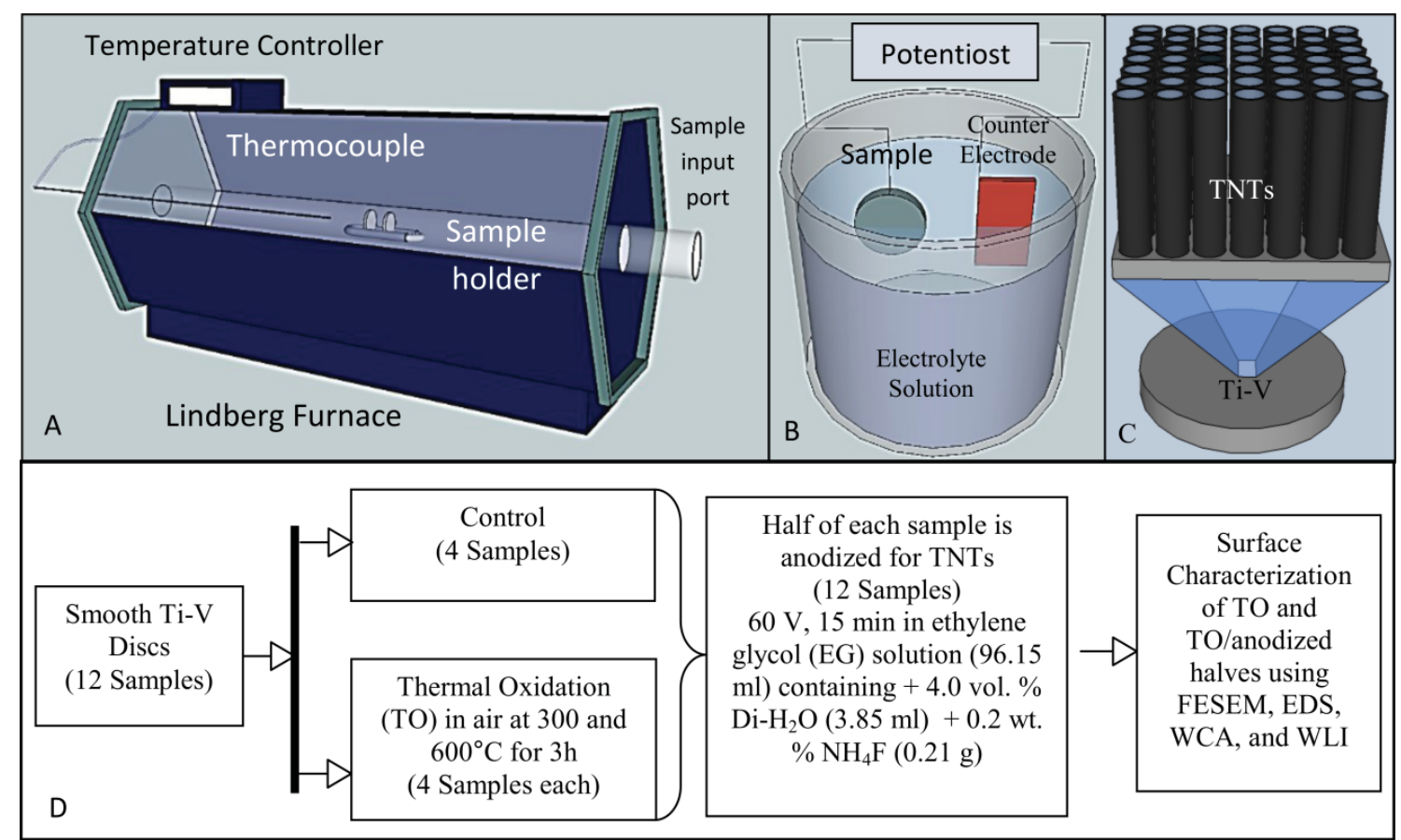

Figure 6.1 Schematic with labeled parts of the A) Lindberg furnace B) anodization setup, C) nanotubes, and D) experimental protocol.

A Lindberg furnace (S\# 54032) was used for TO-treatments; a k-type thermocouple was placed at the center of the furnace quartz tube (35 $\mathrm{cm}$ from opening) and connected to a temperature controller (JLD-612) to maintain working temperatures of 300 and $600{ }^{\circ} \mathrm{C}$ (figure 6.1A). The samples were loaded into the quartz tube to the center of the furnace at a gradual insertion rate of $5 \mathrm{~cm}$ every 5 minutes to prevent micro-cracks from thermal shock. Similarly, samples were gradually extracted after TO-treatment at a rate of $5 \mathrm{~cm}$ every 5 minutes to prevent micro-cracks from an abrupt cool-down. Using existing computational and experimental data, diffusion depth of oxygen was calculated for the working temperatures of 300 and $600{ }^{\circ} \mathrm{C}$.[298-300] Based on calculations, oxygen is expected to diffuse $60 \mathrm{~nm}$ into Ti-V during the 3 hour $300{ }^{\circ} \mathrm{C}$ TO-treatment and 2000 $\mathrm{nm}$ into Ti-V during the 3 hour $600{ }^{\circ} \mathrm{C}$ TO-treatment. Between $10-20 \%$ of the calculated 
diffusion depth is expected to be composed of $\mathrm{TiO}_{2}$, or $12 \mathrm{~nm}$ and $400 \mathrm{~nm}$ for 300 and $600{ }^{\circ} \mathrm{C}$ TO-treatment, respectively.[301]

For anodization, a voltage source (Keithley 2400 SourceMeter) was connected to Ti-V samples (working electrode) and a copper counter electrode (figure 6.1B) in electrolyte of ethylene glycol (EG, $96.15 \mathrm{ml}), 4.0$ vol. \% DI-water $(3.85 \mathrm{ml})$, and $0.2 \mathrm{wt}$. $\% \mathrm{NH}_{4} \mathrm{~F}(0.21 \mathrm{~g})$. Ti-V samples were anodized at $60 \mathrm{~V}$ at room temperature for $15 \mathrm{~min}$. After anodization, samples were washed with DI-water, air dried and wrapped in sterile tissue (Kimwipe, Kimtech Science) and stored in a glass petri dish (KIMAX ${ }^{\circledR}$ Petri Dish).

The experimental groups are control, $300{ }^{\circ} \mathrm{C}$ TO-treated (TO-300), and $600{ }^{\circ} \mathrm{C}$ TO-treated (TO-600), control/anodized (CTNT), $300{ }^{\circ} \mathrm{C}$ TO/anodized (300TNT), and $600{ }^{\circ} \mathrm{C}$ TO-anodized (600TNT).

\section{Surface Characterization}

Sample surface characterization was carried out by means of Field Emission Scanning Electron Microscopy (FESEM), Energy Dispersive X-Ray Spectroscopy (EDS), Water Contact Angle (WCA) measurements, and White Light Interferometry (WLI).

(a) Field Emission Scanning Electron Microscope (FESEM)

A JEOL JSM-6320F FESEM was used to characterize sample surfaces after TO and TO/anodization treatments. Anodized sample surfaces were scraped to remove TNTs onto double sided conductive carbon tape which was mounted on an aluminum stub for imaging. All TNT images were produced with 30k magnification and a scale of $100 \mathrm{~nm}$. ImageJ software was used to measure TNT dimensions.

(b) Energy Dispersive X-Ray Spectroscopy (EDS)

An EDS attachment of the JEOL JSM-6320F FESEM was used for the chemical characterization of TO-treated and TO/anodized surfaces. Random locations on 
control and TO-treated samples and the top, center, and bottom of randomly selected TNTs from anodized surfaces were analyzed. All scans were carried out with $30 \mathrm{k}$ magnification. An acceleration voltage of $10 \mathrm{keV}$ was used for all EDS scans; emission energies of aluminum ( $\mathrm{Al}$ ), titanium (Ti), and vanadium (V) are 1.486, 4.508 and $4.949 \mathrm{keV}$, respectively, and two times the highest emission energy of elements of interest is recommended.

(c) Water Contact Angle (WCA) Measurements

A micro-syringe (Hamilton, 802RN) was used to place $5 \mu \mathrm{L}$ DI-water sessile droplets on TO-treated and TO/anodized Ti-V surfaces and a goniometer (Rame'-Hart NRL CA) was used to measure the WCA of the droplets. After TO and $\mathrm{TO} /$ anodization treatments, all samples were wrapped in sterile tissue for storage. Before WCA measurement, samples were removed from storage and sprayed with $\mathrm{N}_{2}$ gas for 30 seconds to remove sterile tissue residue. After WCA measurements, samples were returned to sterile tissue wrap for storage. WCA measurements were taken at the following stages: 1) immediately after TO-treatment, 2) after a four week aging period following TO-treatment, 3) after DI-water wash following the aging

period , 4) immediately after anodization, 5) over a two week aging period following anodization on Day 1, 2, 7 and 14, and 6) after DI-water wash following Day 14 of aging period. For the aging period TO/anodized samples (stage 5), samples were removed from sterile tissue wrap and sprayed with $\mathrm{N}_{2}$ gas for 30 seconds to remove residue prior to WCA measurements. After WCA measurements, samples were returned to sterile tissue wrap for storage.

(d) White Light Interferometry (WLI)

WLI (NewView 6300, Zygo Corporation) was used to carry out surface roughness measurements before and after TO-treatment, provided as average roughness values $\left(\mathrm{R}_{\mathrm{A}}\right)$. Surface roughness measurements were not carried out on anodized samples. Due to the morphology of TNTs, light does not propagate to the bottom of the TNT structure, therefore accurate roughness measurements cannot be taken.

Statistical Analysis 
OriginPro 9.0 was used for Tukey one-way ANOVA to determine statistical significance in surface wettability, surface roughness, and TNT dimensions. For all analysis, $p<0.05$ was considered statistically significant.

\subsection{Results}

FESEM images of TO-treated and TO/anodized samples are presented in figure 6.2. No difference in surface roughness was observed for control and TO-300 samples (figure $6.2 \mathrm{~A}$ and $6.2 \mathrm{~B}$, respectively); a difference in surface roughness was observed between control and TO-600 samples (figure 6.2A and 6.2C, respectively). WLI data verifies the FESEM images of control, TO-300, and TO-600 surfaces (figure 6.3); $\mathrm{R}_{\mathrm{a}}$ was measured to be $10 \pm 2,11 \pm 2$, and $29 \pm 4 \mathrm{~nm}$ for control, TO-300, and TO-600, respectively. While surface pores were visible on control and 300TNT surfaces, very few pores were visible on 600TNT surfaces, forming a cover layer with TNTs present underneath (figure 6.4A-C). 


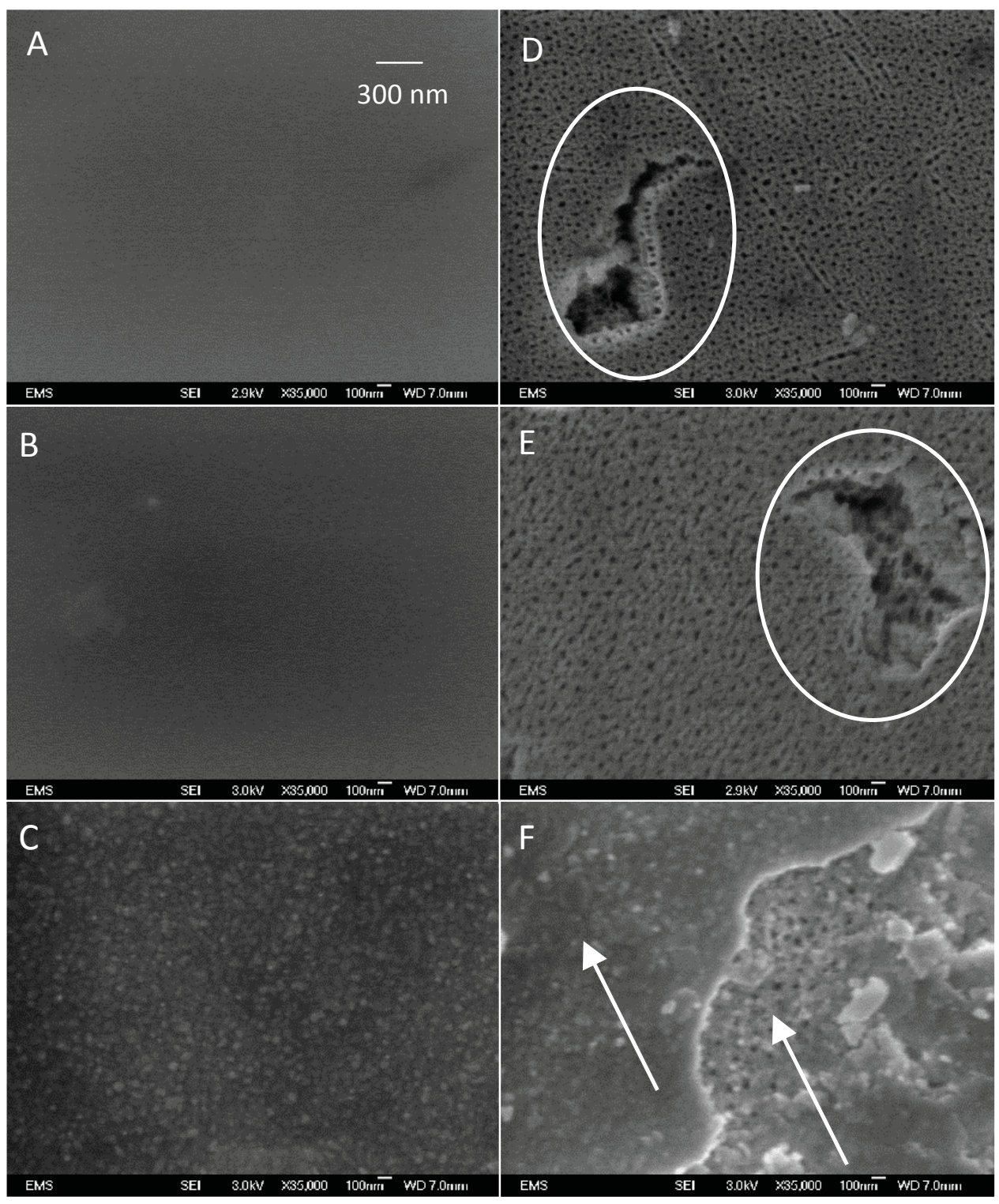

Figure 6.2 Field Emission Scanning Electron Microscopy (FESEM) images of before and after anodization treatments: images $\mathrm{A}$ and $\mathrm{D}$ - control and control/anodized (CTNT) sample, respectively; images $\mathrm{B}$ and $\mathrm{E}-300{ }^{\circ} \mathrm{C}$ TO-treated (TO300) and $300{ }^{\circ} \mathrm{C}$ TO/anodized (300TNT), respectively; images $\mathrm{C}$ and $\mathrm{F}-600{ }^{\circ} \mathrm{C}$ TO-treated (TO600) and $600{ }^{\circ} \mathrm{C} \mathrm{TO}$ /anodized (600TNT), respectively. Effect of TO-treatment before anodization is observed in the form of reduced and smaller surface pores (300TNT and 600TNT samples, images E and F, respectively). On majority of 600TNT surface, a cover layer is present as seen in image F. In images D and E, circled areas represent dissolution of vanadium rich areas. 
At the top of all TNTs, a fuzzy-textured layer $100 \pm 15 \mathrm{~nm}$ thickness was observed (figure 6.4). TNT separation within this layer was less distinct and surface pore diameters (surface inner diameter $=$ SID) were smaller compared to inner diameters below this layer (ID).

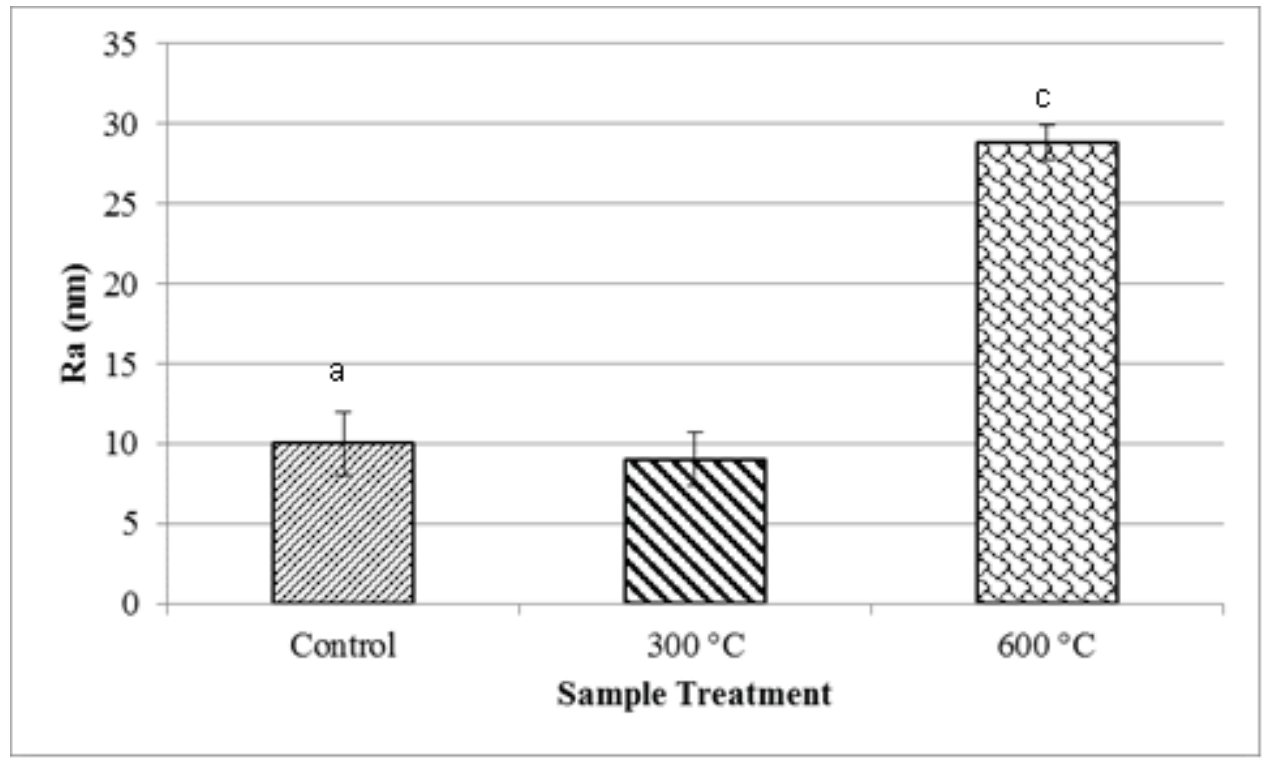

Figure 6.3 White Light Interferometry (WLI) data shows an increase in roughness for TO600 samples over control and TO300 samples. The data corroborates with FESEM images of TO-treated surfaces (figure 6.2). Statistical markers: $\mathrm{a}=$ control, $\mathrm{c}=$ significant difference between TO600 and control samples with $p<.05$. 

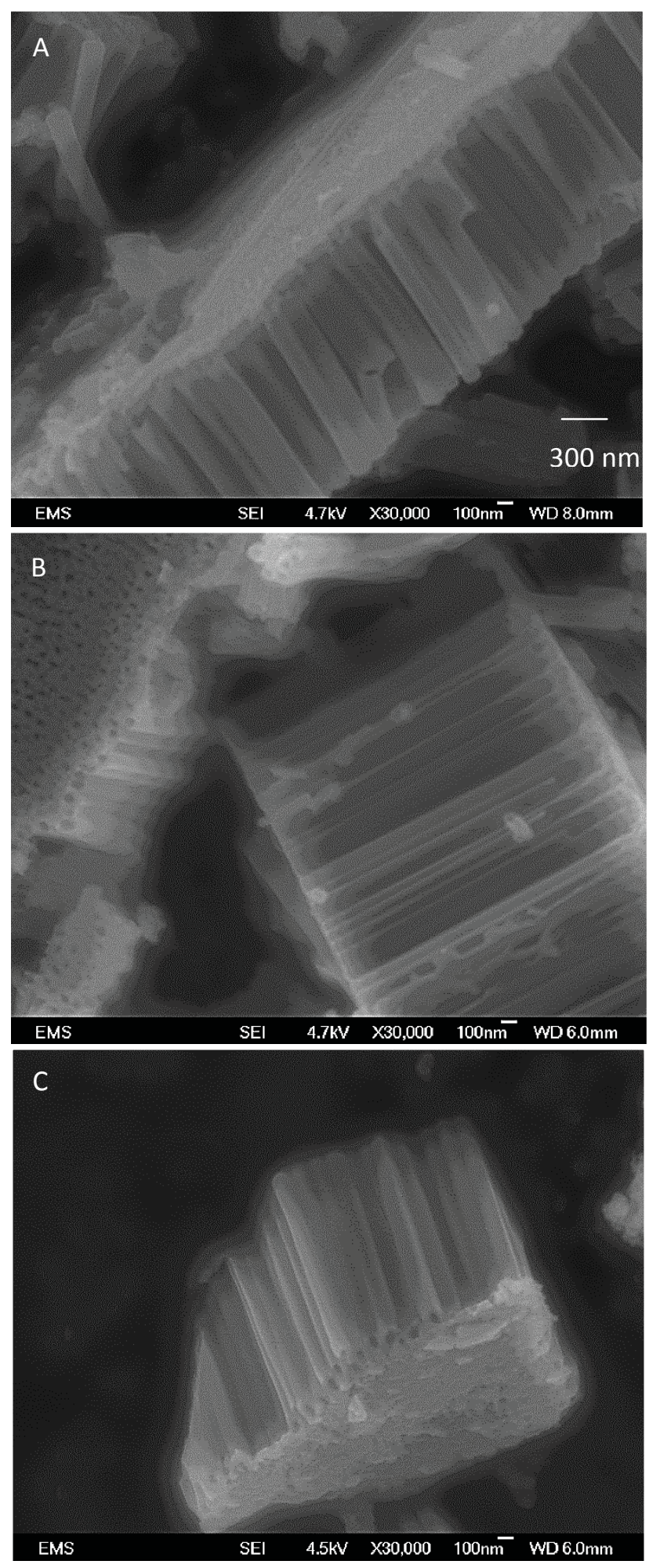

Figure 6.4 FESEM images of $\mathrm{TiO}_{2}$ nanotubes (TNT) from A) CTNT, B) 300TNT, and C) 600TNT samples. Longer TNTs were observed on 300TNT samples as compared to CTNT and 600TNT samples. 
EDS was used to quantify the elemental composition of TO/anodized surfaces. Scans of top, center, and bottom of randomly picked TNTs were conducted and no difference in elemental composition was found. TNT O:Ti ratios of 2.0, 2.1, and 1.8 were seen for CTNT, 300TNT, and 600TNT surfaces, respectively indicating presence of $\mathrm{TiO}_{2}$ in all of the analyzed TNTs. An example EDS scan is given in figure 6.5. EDS of TOtreated surfaces was not successful.

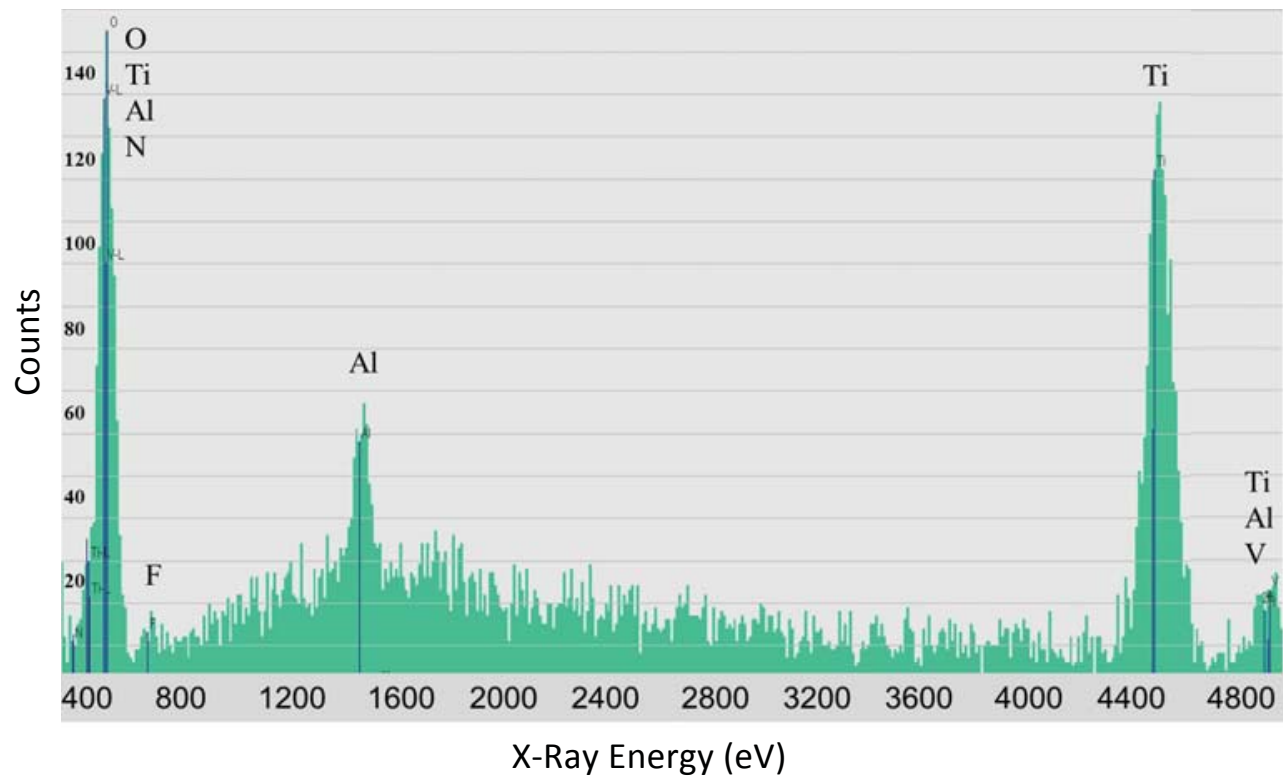

Figure 6.5 Energy Dispersive X-ray Spectroscopy of TNT taken from a 600TNT sample. Titanium, aluminum, vanadium, nitrogen, and fluorine are labeled. Ti, Al, and $\mathrm{V}$ are from Ti- $\mathrm{V}$ components, with the oxygen from $\mathrm{TiO}_{2}$ nanotubes, and residual fluorine and nitrogen from electrolyte and/or presence of fluorine on TNTs in the form of $\mathrm{TiF}_{6}{ }^{-2}$. Spectra from all anodized samples showed calculated oxygen to titanium ratio of 2:1 $\left(\mathrm{TiO}_{2}\right)$.

Measurements of TNT length, surface inner diameter, inner diameter, and outer diameter (OD) are given in figure 6.6. Surface inner diameters were measured as $30 \pm 8$ and $34 \pm 7 \mathrm{~nm}$ for CTNT and 300TNT groups, respectively, and surface inner diameters on 600TNT samples were too few and too small to be accurately measured. 


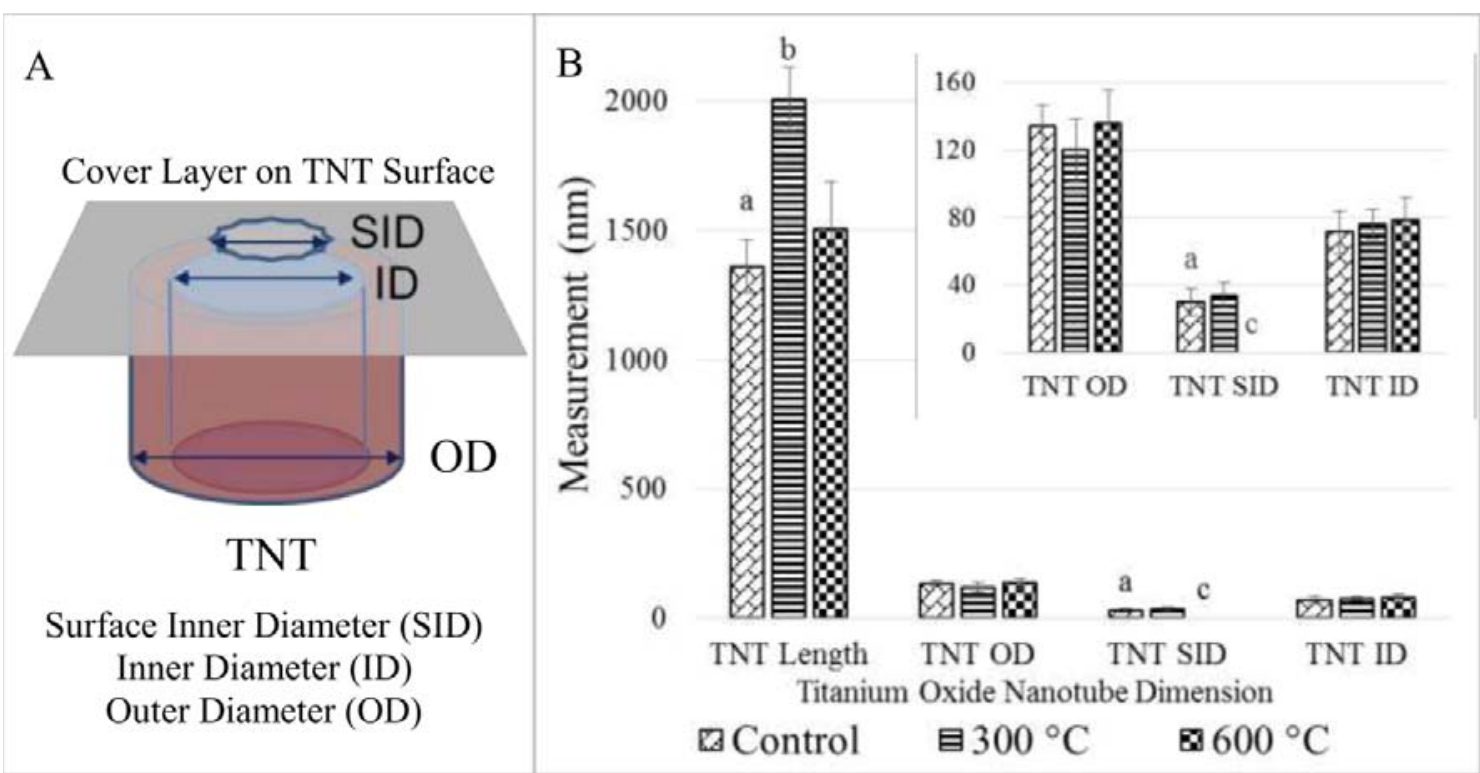

Figure 6.6 Dimensions of TNTs. A) ImageJ was used to measure the TNT length, surface inner diameter (SID), and inner/outer diameters under the surface (ID and OD). B) Graph of TNT dimensions. INSET: Blowup of OD, SID, and ID. Statistical markers with $p<$ $.05: \mathrm{a}=$ control, $\mathrm{b}=$ significant difference in TNT lengths between CTNT and 300TNT samples, $\mathrm{c}=$ significant difference in SID between CTNT and 600TNT samples.

While the surface inner diameters of CTNT and 300TNT are similar, surface pores were visibly fewer on 300TNT. Inner diameters under the surface were not significantly different between the groups $(72 \pm 13,76 \pm 8$, and $78 \pm 14 \mathrm{~nm}$ for CTNT, $300 \mathrm{TNT}$, and 600TNT groups, respectively). No significant difference was observed in the outer diameter under the surface between the groups $(134 \pm 13,120 \pm 19$, and $136 \pm$ $20 \mathrm{~nm}$ for CTNT, 300TNT, and 600TNT groups, respectively). TNT lengths of 300TNT group $(2.01 \pm .12 \mathrm{um})$ were observed to be significantly longer as compared to CTNT and 600TNT groups $(1.36 \pm .10 \mathrm{um}(p<0.001)$ and $1.51 \pm .18$ um $(p<0.001)$, respectively). 


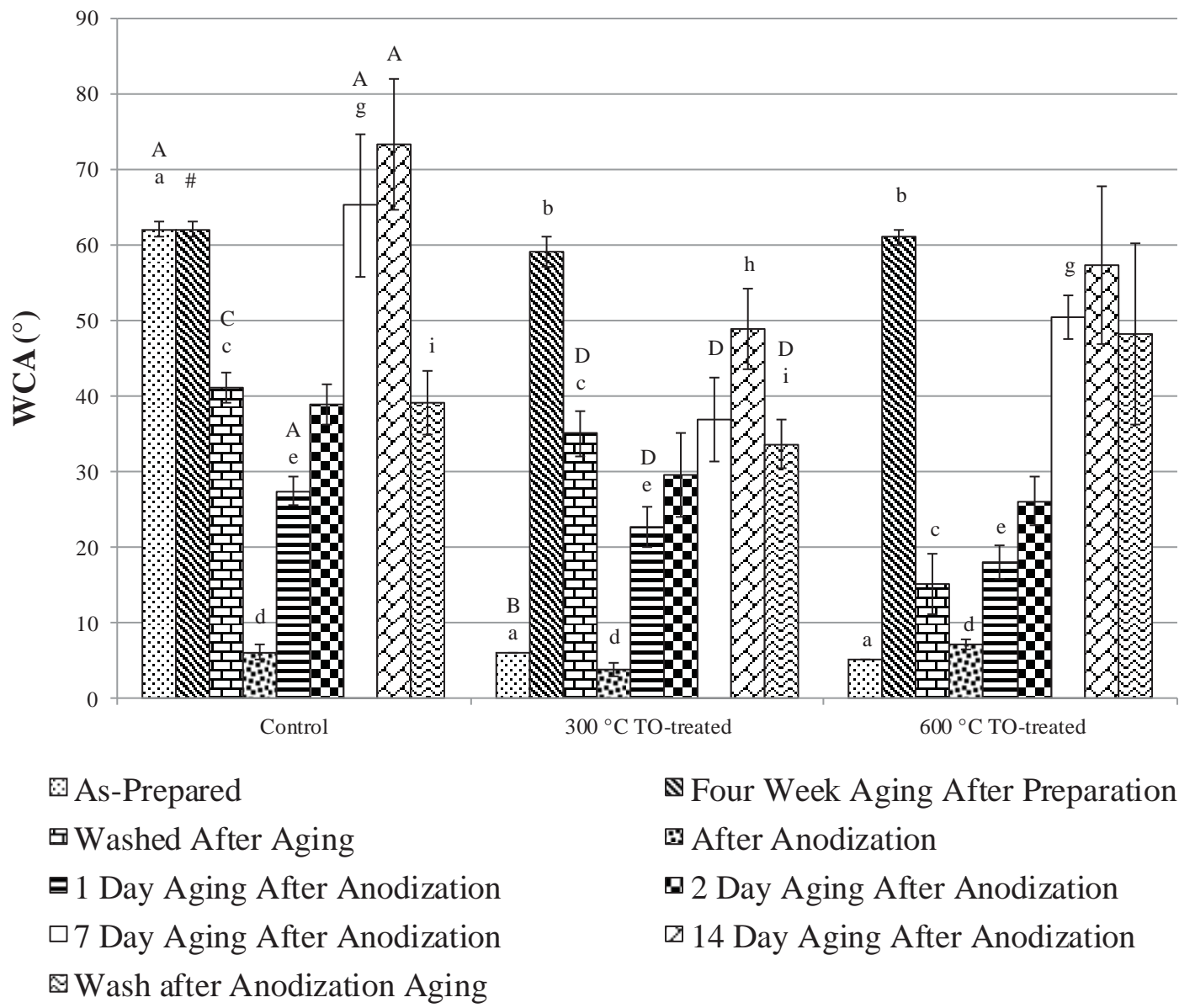

Figure 6.7 Water Contact Angle (WCA) measurements taken after TO and $\mathrm{TO} /$ anodization treatments. $\mathrm{TO}$ and $\mathrm{TO} /$ anodization treatments result in significantly hydrophilic surfaces; WCA increases significantly over time (aging) for all groups; and WCA decreases significantly after DI-water wash for all groups except for 600TNT samples. Lower-case letters are assigned for statistical analysis within each group and upper-case letters are assigned for analysis between groups. Statistical markers with $p<$ .05: $\mathrm{a}=$ control, $\#=$ as-prepared and aged control samples are the same, $\mathrm{b}=$ significant difference between as-prepared and aged samples, $\mathrm{c}=$ significant difference between aged and washed-after-aging samples, $\mathrm{d}=$ significant difference between washed-afteraging and anodized samples, e = significant difference between anodization and 1 Day aging, $\mathrm{f}=$ significant difference between 1 and 2 Day aging, $\mathrm{g}=$ significant difference 
between 2 and 7 Day aging, $\mathrm{h}$ = significant difference between 7 Day and 14 Day aging, and $\mathrm{i}=$ significant difference between 14 Day aging and wash-after-aging.

WCA measurements for TO-treated and TO/anodized samples are given in figure 6.7. Immediately after $\mathrm{TO}$ and $\mathrm{TO} /$ anodization treatments, hydrophilic behavior was observed on all sample surfaces $\left(<10^{\circ}\right)$. Aging, however, increased hydrophobic behavior for both TO-treated and TO/anodized groups. Hydrophobic behavior was observed for TO-treated samples within days and remained steady up to four weeks for all TO-treated groups $\left(61 \pm 2^{\circ}\right)$. DI-water wash after the aging period led to a decrease in WCA to $41 \pm 2^{\circ}(p<0.05), 35 \pm 3^{\circ}(p<0.05)$, and $15 \pm 4^{\circ}(p<0.001)$ for control, TO300 , and TO-600 samples, respectively. Over two weeks, 300TNT and 600TNT groups aged to lesser extents $\left(49 \pm 5^{\circ}\right.$ and $57 \pm 11^{\circ}$, respectively) than the CTNT group (73 \pm $\left.9^{\circ}\right)$. For CTNT and 300TNT samples, DI-water wash after the aging period led to a significant decrease in WCA to $39 \pm 4^{\circ}(p<0.001)$ and $34 \pm 3^{\circ}(p<0.001)$, respectively. DI-water wash after the aging period of $600 \mathrm{TNT}$ samples did not lead to a significant decrease; WCA decreased from $57 \pm 11^{\circ}$ to $48 \pm 12^{\circ}$.

\subsection{Discussion}

\section{Surface Roughness}

FESEM images and WLI data of TO-treated samples (figures 6.2A-C and 6.3, respectively) indicate a correlation between TO-treatment temperature and roughness as seen in literature.[309] Previously, majority anatase and majority rutile in TO-300 and TO-600 Ti-V samples, respectively, were observed with Fourier Transform Infrared (FTIR) spectroscopy (not shown). Surface roughness value of TO-600 surface was significantly higher $(p<0.001)$ than control and TO-300 surfaces because rutile $\mathrm{TiO}_{2}$ formation results in significant changes in surface morphology while no changes occur for as-is amorphous and anatase $\mathrm{TiO}_{2}$ of TO-300 samples.

\section{Variations in Elemental Composition}


EDS was used to confirm the elemental composition of the TO-treated and TO/anodized surfaces: there was no observable difference in the TNT composition between the anodized groups. O:Ti ratio was calculated to be 2.0, 2.1, and 1.8 for CTNT, 300TNT, and 600TNT, respectively, indicating presence of $\mathrm{TiO}_{2}$. EDS of TO-treatedonly surfaces was not possible due to the low thickness of the $\mathrm{TiO}_{2}$ layers; the electron beam ( $\mathrm{e}^{-}$-beam) of $600 \mathrm{~nm}$ was greater than the oxides of control and TO-treated surfaces, therefore only the bulk Ti-V components ( $\mathrm{Ti}, \mathrm{Al}$, and $\mathrm{V}$ ) were present in the spectrums.[310,311]

\section{Variations in Water Contact Angle}

WCA measurements carried out immediately after TO-treatment were superhydrophilic $\left(\mathrm{WCA} \leq 5^{\circ}\right.$ ) as expected (figure 6.7).[272-275] Crystalline structure of $\mathrm{TiO}_{2}$ promotes dissociation of $\mathrm{H}_{2} \mathrm{O}$ molecules, leading to hydroxylation of the surface.[273,312,313] Hydroxylation attracts $\mathrm{H}_{2} \mathrm{O}$ molecules, forming $\mathrm{OH}-\mathrm{H}_{2} \mathrm{O}$ complexes which are water adsorption sites.[313] Stored control and TO-treated samples experienced hydrophobic behavior, or aging, $\left(\mathrm{WCA}=61 \pm 2^{\circ}\right)$ as reported in literature [314] with a significant decrease in WCA after DI-water wash; WCA decreases to $41 \pm$ $2^{\circ}(p<0.05), 35 \pm 3^{\circ}(p<0.05)$, and $15 \pm 4^{\circ}(p<0.001)$ for control, TO-300, and TO600 samples, respectively. Similar to hydroxylation, $\mathrm{TiO}_{2}$ crystallinity also delays dehydroxylation (aging) of the surface $\mathrm{TiO}_{2}$ due to the higher affinity to maintain hydroxylation. This phenomenon is also evident in TO/anodized samples and is discussed subsequently.

As can be seen in figure 6.7, TO/anodization treatments also produced hydrophilic surfaces $\left(<10^{\circ}\right)$. Anodized samples show hydrophilic behavior due to the increased surface area available for water contact on the nanotubes.[6,56,174] While aged anodized groups displayed hydrophobic behavior, 300TNT and 600TNT groups aged to a lesser extent $\left(49 \pm 5^{\circ}\right.$ and $57 \pm 11^{\circ}$, respectively) compared to the CTNT group $\left(73 \pm 9^{\circ}\right)$. In addition, the hydrophobic behavior of TO-treated samples has been observed within a few days as compared to the gradual increase in hydrophobic behavior of TO/anodized groups over two weeks; hydroxylation and dehydroxylation equilibrium takes longer to reach on the greater TNT surface area. It is reported, however, that a nano-structured 
hydrophobic surface attracts and traps air[315]; contact between $\mathrm{TiO}_{2}$ and $\mathrm{H}_{2} \mathrm{O}$ is prevented therefore hydrophobic behavior is expected to continue to increase.[315,316] It may also be possible that the 300TNT TNTs may be of crystalline nature, therefore diminishing the aging process [272-275] as compared to CTNT and 600TNT; the observed difference in surface morphology (figure 6.2D-F) may also be due to the crystalline $\mathrm{TiO}_{2}$.

DI-water wash after the aging period led to a significant decrease in WCA for CTNT and 300TNT samples (WCA decreased from $73 \pm 9^{\circ}$ and $49 \pm 5^{\circ}$ to $39 \pm 4^{\circ}(p<$ $0.001)$ and $34 \pm 3^{\circ}(p<0.001)$, respectively). The greater surface area of CTNT and 300TNT surfaces is attributed to the decrease in the WCA; CTNT and 300TNT afterwash WCA are similar, indicating an equivalent air entrapment behavior which limits how low WCA decreases, irrespective of the anatase or amorphous TNT structure. Decrease in WCA after DI-wash for 600TNT samples was not significant (WCA decreased from $57 \pm 11^{\circ}$ to $48 \pm 12^{\circ}$ ); reduced hydroxylation from DI-water wash due less surface area available on 600TNT surface.

\section{TNT Morphology}

A denser distribution of surface pores is observed on the CTNT surface as compared to $300 \mathrm{TNT}$ surface (figures 6.2D and 6.2E); the 600TNT surface has very few surface pores and is nearly covered by a $\mathrm{TiO}_{2}$ cover, possible remnants of the TO-formed $\mathrm{TiO}_{2}$ (figure 6.2F). TNTs formed on 300TNT surfaces acquire lengths of $2.01 \pm .12 \mu \mathrm{m}$ as compared to $1.36 \pm .10 \mu \mathrm{m}$ and $1.51 \pm .18 \mu \mathrm{m}$ for TNTs on CTNT and 600TNT surfaces, respectively. As seen in figure 6.8, an equilibrium between oxidation/dissolution of the BOL in the presence of fluoride ion leads to the formation of TNTs (see steps 4 and 5 below).[6,305] For the oxidation part, a "barrier-like oxide layer" (BOL) formed as a precursor to TNT formation, may explain the difference morphology (figure 6.8A).[6,81,282,304,305,314] From Kowalski et al., Minagar et al., and Chen et al., TNTs likely form due to the following anodic reactions occurring at the $\mathrm{Ti} /$ oxide and oxide/electrolyte interfaces:

$$
\mathrm{H}_{2} \mathrm{O}(\mathrm{l}) \rightarrow \mathrm{H}^{+}+\mathrm{OH}^{-}
$$




$$
\begin{aligned}
& \mathrm{OH}^{-} \rightarrow \mathrm{H}^{+}+\mathrm{O}^{2-} \\
& \mathrm{Ti}(\mathrm{s})+4 \mathrm{OH}^{-} \rightarrow \mathrm{Ti}(\mathrm{OH})_{4}+4 \mathrm{e}^{-} \\
& \mathrm{Ti}(\mathrm{s})+2 \mathrm{O}^{2-} \rightarrow \mathrm{TiO}_{2}(\mathrm{~s})+4 \mathrm{e}^{-} \\
& \mathrm{TiO}_{2}(\mathrm{~s})+6 \mathrm{~F}^{-}+4 \mathrm{H}^{+} \rightarrow\left[\mathrm{TiF}_{6}\right]^{2-}(\mathrm{s})+2 \mathrm{H}_{2} \mathrm{O}
\end{aligned}
$$

At the end, TNTs are composed of a mixture of $\mathrm{TiO}_{2}$ and $\mathrm{Ti}(\mathrm{OH})_{4}$; the dehydration of anodized samples after removal from electrolyte leads to TNT separation.[306] In preliminary work, the BOL is observed to form during the first five minutes of anodization until the oxidation/dissolution equilibrium condition is met (figure 6.8C). $[6,43,305,306]$ Compared to CTNT samples, a shorter BOL formation step or accelerated TNT formation may explain the longer TNTs of 300TNT samples. $\mathrm{H}^{+}$ion production/accumulation occurs during $\mathrm{BOL}$ formation, attracting $\mathrm{F}^{-}$ions necessary for dissolution of the BOL surface to form nanotubular structure (figure 6.8A). $[6,43,305,306]$ Dissolution of the BOL at the bottom of nanotubular structure exposes the underlying bulk $\mathrm{Ti}-\mathrm{V}$ to the electrolyte where new $\mathrm{TiO}_{2}$ complexes are formed (figure 6.8C) and the cycle of oxditation/dissolution is repeated. $[6,43,305,306]$ Namely there are two steps in which TO-formed $\mathrm{TiO}_{2}$ may affect anodization mechanism: i) $\mathrm{H}^{+}$production and ii) the transport of $\mathrm{F}^{-}$ions to $\mathrm{TiO}_{2}$ complexes. 
A

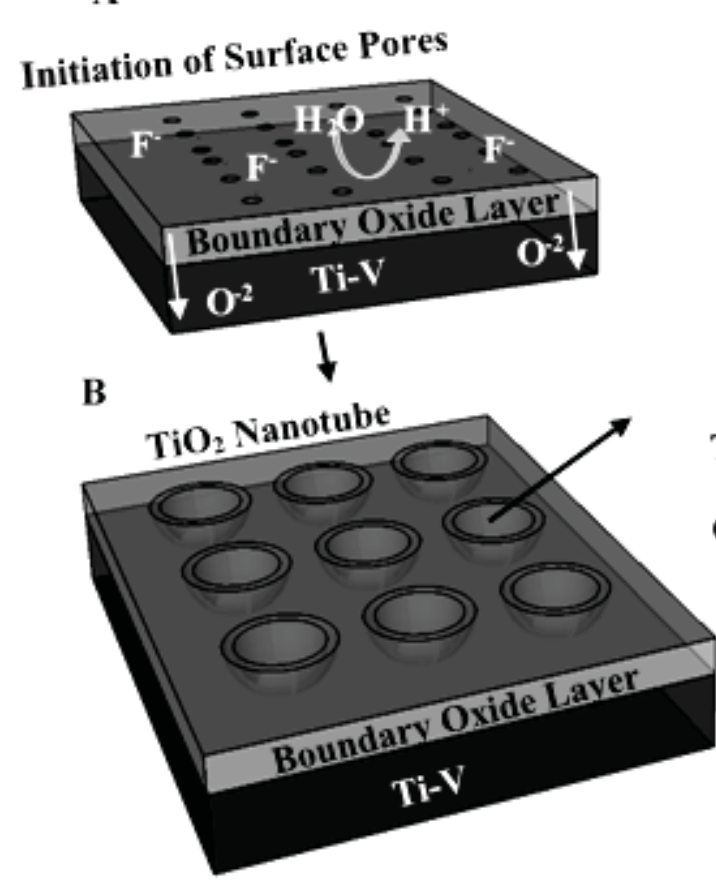

C

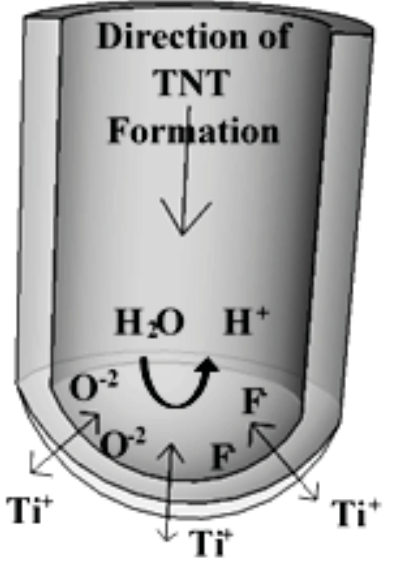

Oxidation-Dissolution

Mechanism

Figure 6.8 Schematic of anodization process. A) Oxidation at the electrolyte and Ti-V surface leads to formation of barrier oxide layer (BOL) and accumulation of $\mathrm{H}^{+}$ions that attract $\mathrm{F}^{-}$ions, resulting in etching or dissolution of BOL, B) etching leads to formation of nano-pores which grow into nanotubes, and C) as oxidation/dissolution steps in part A and $\mathrm{B}$ reach equilibrium at the Ti-V bulk and nanotube interface, nanotubes extend into Ti-V bulk.

\section{i) $\mathrm{H}^{+}$production and accumulation}

Compared to Ti-V with amorphous $\mathrm{TiO}_{2}$, the increased $\mathrm{H}_{2} \mathrm{O}$ dissociation on anatase and rutile surfaces of TO-300 and TO-600 surfaces, respectively, may produce more $\mathrm{H}^{+}$ions, attract more $\mathrm{F}^{-}$ions, and lead to oxidation/dissolution equilibrium quicker.[273,312,313] While this explains longer TNTs on 300TNT surface, FESEM images of the 300TNT and 600TNT surfaces (figure 6.2E and 6.2F) show insufficient dissolution on the surface; crystallinity is not the only factor in the observed difference in TNT morphology. The oxide formed by $600{ }^{\circ} \mathrm{C}$ TO-treatment may be too thick, delaying 
the point at which oxidation/dissolution equilibrium is reached; $400 \mathrm{~nm}$ of $\mathrm{TiO}_{2}$ and 1600 $\mathrm{nm}$ of oxygen diffusion zone are expected for $600{ }^{\circ} \mathrm{C}$ TO-treatment.[301] The oxygen diffusion zone has less than the 2:1 O:Ti ratio. Due to the oxide and diffusion zone, oxidation may occur at lower rates (reduced $\mathrm{H}^{+}$ion production), dissolution is dominant, and therefore oxidation/dissolution equilibrium is delayed. As a result, TNTs on 600TNT samples are shorter as compared to TNTs on 300TNT samples. TNTs on 600TNT samples are longer than CTNT samples, however, suggesting that anodization is faster due to the rutile $\mathrm{TiO}_{2}$ once oxidation/dissolution equilibrium is reached.

\section{ii) F' ion Transport}

Crystalline $\mathrm{TiO}_{2}$ is known to be more reactive than amorphous $\mathrm{TiO}_{2}$ and diffusivity of $\mathrm{TiO}_{2}$ increases when doped with non-metals such as fluorine.[317-321] The minority anatase $\{001\}$ facets experience dissociative adsorption of reactant molecules, are preferentially fluorinated, and therefore energetically stabilized.[322,323] Fterminated $\{001\}$ facets reduce the activation energy of reactant molecules and affect the reaction mechanism at the molecular level.[322,324] In addition, Wagemaker et al. suggests that diffusion of foreign ions into anatase occurs at a higher rate. Although rutile $\mathrm{TiO}_{2}$ has higher diffusion rates for distances of $\mu \mathrm{m}$ 's when compared to amorphous and anatase $\mathrm{TiO}_{2}, \mathrm{~F}^{-}$ion transport is reduced for larger distances due ineffective grain stacking (grain boundaries); the dissolution mechanism is therefore slowed and oxidation/dissolution equilibrium is delayed. [317,319]

\section{Surface Pores}

As TNTs become longer, the $\mathrm{F}^{-}$ions accumulate at the bottom of TNTs where new $\mathrm{H}^{+}$ions are produced via $\mathrm{TiO}_{2}$ production and dissolution at the surface ceases.[305,325-327] Based on this phenomena, the more $\mathrm{TiO}_{2}$ that exists before anodization, the more $\mathrm{TiO}_{2}$ will remain after anodization as this fuzzy layer. Even the highly water-dissociative property of rutile structure formed during $600{ }^{\circ} \mathrm{C}$ TO-treatment is not sufficient in dissolution of the thick $\mathrm{TiO}_{2}$ layer; water dissociation produces $\mathrm{H}^{+}$ 
ions which attract $\mathrm{F}^{-}$ions for dissolution of $\mathrm{TiO}_{2}$. Increasing the water content in the electrolyte solution, however, may accelerate the removal of this fuzzy layer before oxidation/dissolution equilibrium is reached.[305,325-327]

\subsection{Conclusions}

In summary, rutile $\mathrm{TiO}_{2}$ may provide the best wettability behavior for improved cellular response. While anatase $\mathrm{TiO}_{2}$ (TO-300) produced the longest TNTs, a thin rutile $\mathrm{TiO}_{2}$ layer may produce even longer TNTs. To compensate the reduced anodization on thick rutile $\mathrm{TiO}_{2}$ (TO-600), we may have to use $\mathrm{TO}$ duration as short as $15 \mathrm{~min}$ to produce rutile $\mathrm{TiO}_{2}$ on Ti-V with thickness similar to that of TO-300 samples. Surface pore size can be maximized by increasing electrolyte water content to enhance dissolution of $\mathrm{TiO}_{2}$ on the TNT surface. The combined benefit of increased TNT lengths, surface pores, and improved wettability can improve cellular response, shelf-life of dental and orthopaedic implants, and create opportunities for drug and cellular media loading applications.

\section{Acknowledgements}

We are grateful to the American Academy of Implant Dentistry Research Foundation for their generous funding to Arman Butt. We are also thankful to the National Science Foundation (CMS \# 0609377, CBET \# 1067424 and EEC \# 1062943)

and the University of Illinois at Chicago, Bioengineering Department for financial support. 


\title{
Chapter 7
}

\section{Thermally oxidized titania nanotubes enhance the corrosion resistance of Ti6Al4V*}

\author{
John Grotberg ${ }^{1,2}$, Azhang Hamlekhan ${ }^{2,3}$, Arman Butt ${ }^{1,2}$, Sweetu Patel ${ }^{1,2}$, Dmitry \\ Royhman $^{1,2}$, Tolou Shokuhfar ${ }^{2,3}$, Cortino Sukotjo ${ }^{2,4}$, Christos Takoudis ${ }^{1,2,5}$, Mathew T. \\ Mathew 2,6 \\ ${ }^{1}$ Department of Bioengineering, University of Illinois at Chicago, 851 S. Morgan St, \\ Chicago, IL, 60607, USA \\ ${ }^{2}$ Institute of Biomaterials, Tribocorrosion and Nanomedicine (IBTN), 851 S. Morgan St, \\ Chicago, IL, 60607, USA \\ ${ }^{3}$ Mechanical Engineering-Engineering Mechanics, Michigan Technological University, \\ 1400 Townsend Dr, Houghton, MI, 49931, USA \\ ${ }^{4}$ Department of Restorative Dentistry, College of Dentistry, University of Illinois at \\ Chicago, 801 S. Paulina, Chicago, IL, 60612, USA \\ ${ }^{5}$ Department of Chemical Engineering, University of Illinois at Chicago, 851 S. Morgan \\ St, Chicago, IL, 60607, USA \\ ${ }^{6}$ Department of Orthopedics, Rush University Medical Center, 1611 W. Harrison, \\ Chicago, IL, 60612, USA
}

\begin{abstract}
*The material contained in this chapter has been submitted to the Journal of Electrochimica Acta. My contribution to this publication includes fabrication of nanotubes, FESEM and reviewing the manuscript.
\end{abstract}




\begin{abstract}
The negative impact of in vivo corrosion of metallic biomedical implants remains a complex problem in the medical field. We aimed to determine the effects of electrochemical anodization $(60 \mathrm{~V}, 2 \mathrm{~h})$ and thermal oxidation $\left(600^{\circ} \mathrm{C}\right)$ on the corrosive behavior of Ti-6Al-4V, with serum proteins, at physiological temperature. Anodization produced a mixture of anatase and amorphous $\mathrm{TiO}_{2}$ nanopores and nanotubes, while the annealing process yielded an anatase/rutile mixture of $\mathrm{TiO}_{2}$ nanopores and nanotubes. The surface area was analyzed by the Brunauer-Emmett-Teller method and was estimated to be 3 orders of magnitude higher than that of polished control samples. Corrosion resistance was evaluated on the parameters of open circuit potential, corrosion potential, corrosion current density, passivation current density, polarization resistance and equivalent circuit modeling. Samples both anodized and thermally oxidized exhibited shifts of open circuit potential and corrosion potential in the noble direction, indicating a more stable nanotube layer, as well as lower corrosion current densities and passivation current densities than the smooth control. They also showed increased polarization resistance and diffusion limited charge transfer within the bulk oxide layer. The treatment groups analyzed can be ordered from greatest corrosion resistance to least as Anodized+Thermally Oxidized $>$ Anodized $>$ Smooth $>$ Thermally Oxidized for the conditions investigated in this study.
\end{abstract}

Key words: Titanium, Corrosion Resistance, Rutile, $\mathrm{TiO}_{2}$ Nanotube, Electrochemical Impedance, Biomedical Implant, Wettability

\title{
7.1 Introduction
}

Titanium and titanium alloy have been widely used in applications for biomedical implants, specifically in hip and dental implants, due to factors of high strength, low modulus, low density, and general resistance to corrosion [328-330]. Release of metal ions from Ti-6Al-4V alloy due to corrosion still remains a concern, as $\mathrm{Al}$ and $\mathrm{V}$ cations 
can cause inflammation and local toxicity, resulting in tissue damage, joint loosening and a need for revision surgeries [331]. Additionally, osseointegration is an integral component of the success of an implant in bone tissue. Because Ti is bioinert, it often shows poor cellular adhesion [332]. In an attempt to alleviate these clinical problems, numerous substrate surface modifications are currently being investigated. The surface modifications of interest regarding Ti-6Al-4V alloy include the formation of $\mathrm{TiO}_{2}$ nanotubes (TNTs) via electrochemical anodization and the formation of thick (compared to native) polycrystalline (anatase/rutile) oxide layers via thermal oxidation (TO). The formation of titania nanotubes via electrochemical anodization is attractive for application due to self-organization in an array perpendicular to the substrate, the ability to control the dimensions with applied voltage and duration, and the ability to couple with other surface treatments such as thermal oxidation (as with this study), coatings, nanotube doping, and drug-loading [333]. These surface modifications have been studied extensively as to their effect on osteogenic response and improved biocompatibility. This response is dependent on surface topography, physiochemistry, mechanics and electronics, and is manifested by changes in adhesion, proliferation, morphology, migration and differentiation of cells in the osteogenic lineage [334]. The presence of nanophase surfaces and amorphous $\mathrm{TiO}_{2}$ nanotubes (30-120 nm in diameter) on $\mathrm{Ti}$ and Ti-alloy substrates have been shown to increase wettability, protein adsorption, cellular adhesion, proliferation and differentiation of osteogenic cells in vitro [334-338]. Similar results regarding accelerated cellular adhesion and differentiation have been seen in vivo as well $[339,340]$. Increased cellular adhesion has also been observed in vitro with thermally oxidized Ti-6Al-4V, and thermally oxidized $\mathrm{TiO}_{2}$ nanotubes, with greater annealing temperature leading to accelerated cellular adhesion. Annealing temperatures $>550^{\circ} \mathrm{C}$ that yield mixtures of anatase and rutile (predominantly rutile) led to greater osteoblast adhesion than amorphous and anatase compact and nanotube surfaces $[278,283,341,342]$. This effect was attributed not only to the nanoscale cellular cues promoted by the nanotube array, but also by the regularity in crystallinity (whether anatase or anatase/rutile) that led to increased regularity in cytoskeleton structure and filopodia spreading of osteoblasts [342]. 
It has been shown that temperature, $\mathrm{pH}$ and the presence of serum proteins affect the corrosive properties of $\mathrm{Ti}$ and $\mathrm{Ti}$ alloy [343,344]. Additionally, annealing commercially pure titanium (cpTi) and Ti alloy at temperatures yielding a mixture of anatase and rutile phases for at least 8 hours has been shown to increase the resistance to corrosion [345,346]. Both amorphous and annealed $\mathrm{TiO}_{2}$ nanotube foil substrates have also been shown to improve corrosion resistance in electrolyte solutions with and without serum proteins. Quantitative analysis of potentiodynamic and EIS studies have shown decreased corrosion current densities and more noble corrosion and open circuit potentials, as well as increased polarization resistance [347-350]. However, the effect and quantification of increased surface area due to titania nanotubes is not reported in current literature with regard to incorporation into current density, resistance and capacitance measurements. These parameters are important to describe per unit area in order to understand what is happening at the substrate surface. Such analysis and discussion will be considered in this study.

As current research explores surface treatments to enhance implant osseointegration, it is necessary to understand the effects on the corrosion properties at the implant surface in a biofluid and cell culture environment. The present work aimed to test the wettability, cellular adhesion response, and corrosive behavior of Ti-6Al-4V alloy discs with novel surface treatments and electrolyte regarding thermal oxidation temperature and duration, electrochemical anodization voltage and duration, and combining the two processes of electrochemical anodization followed by thermal oxidation, in cell culture media with serum proteins. Additionally, a novel method of surface area analysis was used to incorporate true surface area increases due to the titania nanopore and nanotube arrays in order to describe the effects of substrate polarization on a per unit area basis. In furthering the understanding of the corrosive properties of the aforementioned surface treatments of Ti-6Al-4V alloy, we expected the thermally oxidized titania nanotubes of higher rutile content to display the greatest resistance to corrosion due to the increased surface area of the nanotube array, added stability of the nanotubes through crystalline structure, higher resistance of rutile compared to anatase, and growth of a thick passive oxide layer beneath the nanotube array [333]. 


\subsection{Materials and Methods}

\section{Materials and surface modifications}

Twenty Ti-6Al-4V alloy discs of $15 \mathrm{~mm}$ diameter and $3 \mathrm{~mm}$ thickness (MacMaster Carr, Elmhurst, IL) were mechanically wet-ground using a series of abrasive pads (\#320, \#400, \#600 and \#800) (Carbimet 2, Buehler, Lake Bluff, IL). Samples were then polished using diamond paste (MetaDi 9-micron, Buehler, Lake Bluff, IL) with lubricant (MetaDi Fluid, Buehler, Lake Bluff, IL) on polishing cloth (TexMet polishing cloth, Buehler, Lake Bluff, IL), and brought to mirror finish using colloidal silica polishing suspension (MasterMet, Buehler, Lake Bluff, IL) on polishing cloth (Chemomet I, Buehler, Lake Bluff, IL). Samples were then divided into 4 groups: smooth (control), TO (formation of rutile/anatase by thermal oxidation at $600^{\circ} \mathrm{C}$ for $3 \mathrm{~h}$ ), Ad (formation of amorphous TNTs by electrochemical anodization at $60 \mathrm{~V}$ for $2 \mathrm{~h}$ ) and $\mathrm{Ad}+\mathrm{TO}$ (formation of rutile/anatase TNTs by $60 \mathrm{~V}, 2 \mathrm{~h}$ anodization followed by $600^{\circ} \mathrm{C}$, 3h thermal oxidation).

The thermal oxidation process of the annealed sample group was performed in a Single-zone Quartz Furnace (Lindberg, S\# 54032) in air at ambient pressure. The samples were ultrasonically cleaned in ethanol and dried with nitrogen gas prior to annealing. Once the target temperature of $600^{\circ} \mathrm{C}$ was obtained, samples were loaded $5 \mathrm{~cm}$ per 5 minutes into the region closest to the thermocouple to ensure gradual temperature change within the samples to avoid micro-cracks from thermal shock, and to ensure the accuracy of final sample temperature. The samples remained at the final position in the furnace for 3 hours, and then were removed $5 \mathrm{~cm}$ per 5 minutes to ensure gradual cooling. From the thermal oxidation process, it was expected to obtain a mixture of anatase and rutile as the predominant phases within the oxide layer.

For the electrochemical anodization process, samples were ultrasonically cleaned in acetone for 30 minutes prior to anodization. Ti-6Al-4V were connected to a voltage source (Keithley 2400 SourceMeter) and functioned as the working electrode while a 
copper rod functioned as the counter electrode. The two-electrode system was submerged in electrolyte containing 4.0 vol. \% DI-water ( $3.85 \mathrm{ml}), 0.2 \mathrm{wt} . \% \mathrm{NH}_{4} \mathrm{~F}(0.21 \mathrm{~g})$, and ethylene glycol (EG, $96.15 \mathrm{ml}$ ). Samples were anodized at $60 \mathrm{~V}$ at room temperature for 2 hours. After anodization, samples were washed with DI-water, air dried and wrapped in sterile tissue (Kimwipe, Kimtech Science) and stored in a glass petri dish (KIMAX® Petri Dish).

For the $\mathrm{Ad}+\mathrm{TO}$ sample group, both of the aforementioned processes were used. Samples were first subjected to the electrochemical anodization protocol of $60 \mathrm{~V}$ for 2 hours in 4.0 vol. \% DI-water $(3.85 \mathrm{ml}), 0.2$ wt. \% $\mathrm{NH}_{4} \mathrm{~F}(0.21 \mathrm{~g})$, and ethylene glycol (EG, $96.15 \mathrm{ml}$ ) electrolyte, followed by the thermal oxidation protocol of $600^{\circ} \mathrm{C}$ for 3 hours.

\section{Surface characterization}

(a) Water contact angle (WCA) measurement: A goniometer (Rame-Hart, NRL, CA) was used to measure the WCA of $5 \mu \mathrm{l}$ water droplets placed onto the smooth, TO, Ad, and $\mathrm{Ad}+\mathrm{TO}$ samples via a micro-syringe (Hamilton, $802 \mathrm{RN}$ ). ImageJ software was used to determine to WCA measurements. Before WCA measurement, samples were removed from storage and sprayed with $\mathrm{N}_{2}$ gas for 30 seconds to remove sterile tissue residue. After WCA measurements, samples were returned to sterile tissue wrap for storage. Day 0 was determined to be immediately after treatment for the TO, anodized, and anodized + TO groups. WCA was measured at the following time points in days: $0,1,2,5,7,14$, and 25 . For the aging period all samples were removed from sterile tissue wrap and sprayed with $\mathrm{N}_{2}$ gas for 30 seconds to remove residue prior to WCA measurements. After WCA measurements, samples were returned to sterile tissue wrap for storage.

(b) Fourier transform infrared (FTIR) spectroscopy: Diffuse reflectance FTIR (Nicolet S\#ADU9700221, Thermo Scientific, Waltham, MA, USA) was used to perform surface composition characterization of anodized and thermally oxidized surfaces using the polished control as a background. Prior to the FTIR scans, samples were purged for 1 
hour to minimize noise. The FTIR spectrum ranged from 400 to $4000 \mathrm{~cm}^{-1}$ and was obtained with $2 \mathrm{~cm}^{-1}$ resolution and 512 scans. Deconvolution was carried out using XPS Peak software.

(c) Field emission scanning electron microscopy (FESEM): A JEOL JSM-6320F FESEM was used to characterize sample surfaces after $\mathrm{Ad}, \mathrm{TO}$, and $\mathrm{Ad}+\mathrm{TO}$ treatments. In order to obtain images of the different dimensions of TNTs, the anodized sample surfaces were scraped to remove TNTs onto double-sided conductive carbon tape, which was mounted onto an aluminum stub. Images displaying the length of the TNTs were produced with $10 \mathrm{k}$ magnification and a scale of $1 \mu \mathrm{m}$. Images displaying the top and bottom of TNTs were produced using 50k magnification and a scale of $100 \mathrm{~nm}$. Image J software was used to measure TNT dimensions.

(d) Brunauer-Emmett-Teller (BET): BET surface area analysis was performed on a smooth and anodized sample using a Quantochrome Autosorb (Quantochrome Instruments, Boynton Beach, FL, USA). Krypton was the adsorbate and the sample mass for the Ad group was $0.67 \mathrm{~g}$.

\section{Electrochemical tests}

All electrochemical tests were performed using a potentiostat (SP-240, BioLogic, Claix, France) and a custom made electrochemical cell. Corrosion measurements were recorded using EC-Lab v.10.23 software (BioLogic, Claix, France). A 3-electrode configuration was used with the Ti alloy sample as the working electrode, a standard calomel reference electrode (SCE), and a graphite rod counter electrode. Dulbecco's Modified Eagle Medium (DMEM) + 10\% fetal bovine serum (FBS) (Gibco, Life Technologies, Grand Island, NY, USA) served as the electrolyte solution to mimic cell culture media (volume $=10 \mathrm{~mL}$ ) (Figure 7.1). Prior to testing, all samples were ultrasonically cleaned in acetone for 15 minutes followed by ultrasonic cleaning in deionized $\mathrm{H}_{2} \mathrm{O}$ for 10 minutes. Samples were mounted to the corrosion cell, and the 
electrochemical setup was kept at $37^{\circ} \mathrm{C}$ using a cell culture incubator (WJ501-T, Baxter Scientific Products, Deerfield, IL, USA) throughout electrochemical testing. 


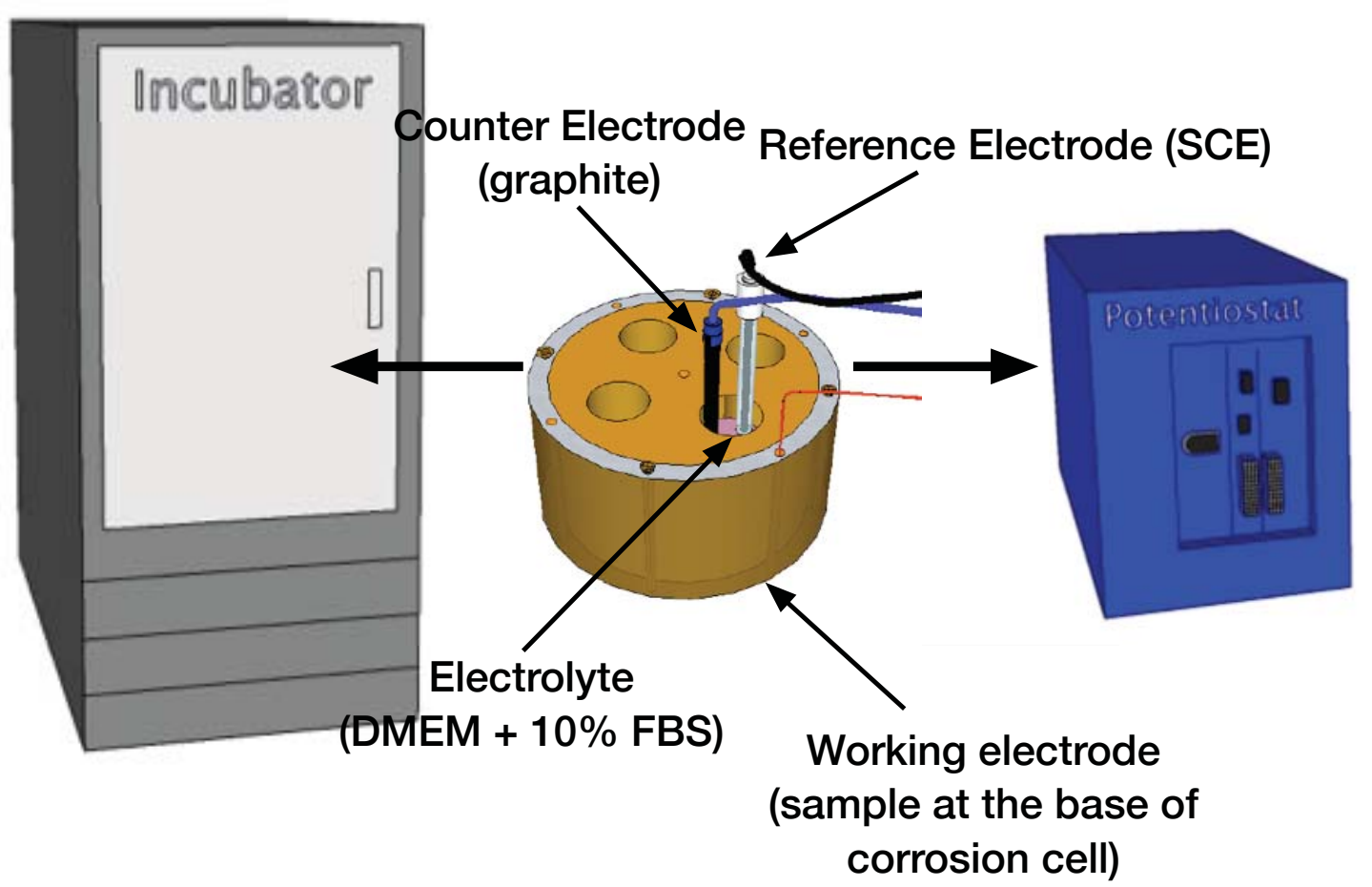

Figure 7.1 Schematic diagram of the experimental apparatus for corrosion testing.

Electrochemical tests performed included open circuit potential (OCP), electrochemical impedance spectroscopy (EIS), and cyclic polarization. Three trials $(n=3)$ were performed for each group. After a potentiostatic electrochemical cleaning stage at $0.9 \mathrm{~V}$ vs. SCE, OCP was performed for 1 hour to allow the system to stabilize. Following OCP, EIS measurements were obtained over a frequency range of $100 \mathrm{~K}-0.005 \mathrm{~Hz}( \pm 10$ $\mathrm{mV}$ vs. SCE at corrosion potential). EIS data was used to determine equivalent circuit models and thereby estimations of effective circuit resistance $\left(\mathrm{R}_{\mathrm{eff}}\right)$ and effective circuit capacitance $\left(\mathrm{CPE}_{\text {eff }}\right)$ using constant phase element (CPE) equivalents. EC-Lab v. 10.23 software was used to perform the z-fit analysis of equivalent circuit models over a range of 1000-0.01 Hz. Nyquist and Bode plots were used to estimate polarization resistance $\left(\mathrm{R}_{\mathrm{p}}\right)$. Finally, samples were cyclically polarized from $-1 \mathrm{~V}$ vs. SCE to $1.8 \mathrm{~V}$ vs. SCE and back to $-1 \mathrm{~V}$ vs. SCE ( $2 \mathrm{mV} / \mathrm{s}$ scan rate). Corrosion parameters evaluated from the cyclic 
polarization scan included corrosion potential $\left(\mathrm{E}_{\text {corr }}\right)$, corrosion current density $\left(\mathrm{I}_{\text {corr }}\right)$ and passivation current density $\left(I_{\text {pass }}\right)$. $E_{\text {corr }}$ and $I_{\text {corr }}$ were estimated using Tafel's method ( \pm $25 \mathrm{mV}$ from $\left.\mathrm{E}_{\text {corr }}\right)$. $\mathrm{I}_{\text {pass }}$ was determined from current density measurements within the passivation region of the cyclic scan.

\section{Statistical analysis}

Wettability was evaluated based on average WCA for each time period of aging. Corrosion behavior was evaluated based on parameters of OCP, $\mathrm{E}_{\text {corr }}, \mathrm{I}_{\text {corr }}, \mathrm{I}_{\text {pass }}, \mathrm{R}_{\text {eff, }}$ $\mathrm{CPE}_{\text {eff }}$ and $\mathrm{R}_{\mathrm{p}}$. Corrosion parameters were tested separately using one-way ANOVA to examine the effects of the different surface treatments. Tukey test analysis was used for pair-wise comparison between treatment groups. Statistical tests were performed using SPSS software (IBM, Armonk, NY, USA) and a critical level of significance of $\mathrm{p}=0.05$.

\subsection{Results}

Properties of the modified surface

(a) WCA: Figure 7.2 shows a graphical analysis of WCA measurements for the various sample groups over 25 days of aging. WCA measurements for the smooth group for days $0,1,2,5,7,14$ and 25 were $35^{\circ} \pm 4^{\circ}, 61^{\circ} \pm 3^{\circ}, 69^{\circ} \pm 9^{\circ}, 69^{\circ} \pm 3^{\circ}, 68^{\circ} \pm 5^{\circ}, 71^{\circ} \pm 4^{\circ}$ and $84^{\circ} \pm 4^{\circ}$ respectively. WCA measurements for the TO group for days $0,1,2,5,7,14$ and 25 were $25^{\circ} \pm 4^{\circ}, 26^{\circ} \pm 4^{\circ}, \quad 34^{\circ} \pm 2^{\circ}, \quad 44^{\circ} \pm 7^{\circ}, 58^{\circ} \pm 6^{\circ}, 70^{\circ} \pm 9^{\circ}$ and $79^{\circ} \pm 5^{\circ}$ respectively. WCA measurements for the Ad group for days $0,1,2,5,7,14$ and 25 were $26^{\circ} \pm 2^{\circ}, 26^{\circ} \pm 5^{\circ}$, $31^{\circ} \pm 3^{\circ}, 37^{\circ} \pm 4^{\circ}, 48^{\circ} \pm 1^{\circ}, 64^{\circ} \pm 5^{\circ}$ and $73^{\circ} \pm 3^{\circ}$ respectively. Finally, WCA measurements for the $\mathrm{Ad}+\mathrm{TO}$ group for days $0,1,2,5,7,14$ and 25 were $7^{\circ} \pm 1^{\circ}, 18^{\circ} \pm 2^{\circ}, 20^{\circ} \pm 3^{\circ}$, $24^{\circ} \pm 1^{\circ}, 28^{\circ} \pm 4^{\circ}, 35^{\circ} \pm 4^{\circ}$ and $43^{\circ} \pm 1^{\circ}$ respectively. WCA analysis of the aged sample groups showed significantly lower contact angles for the $\mathrm{Ad}+\mathrm{TO}$ group compared to smooth throughout the 25 -day aging period $(\mathrm{p}<0.001)$. Compared to the TO group, $\mathrm{Ad}+\mathrm{TO}$ showed significantly lower WCA on days $0(\mathrm{p}<0.001), 2(\mathrm{p}=0.036)$, and 5-25 
( $\mathrm{p}<0.001)$, while compared to the Ad group, Ad+TO showed lower WCA on days 0 $(\mathrm{p}<0.001), 5(\mathrm{p}=0.005)$, and 7-25 $(\mathrm{p}<0.001)$. Additionally, the Ad and TO groups showed significantly lower contact angles compared to smooth for days $0-14(\mathrm{p}=0.03)$, and were not statistically significant from one another throughout the aging process $(p>0.05)$.

(b) FTIR data: The annealing temperature of $600^{\circ} \mathrm{C}$ for 3 hours was expected to yield polycrystalline anatase and rutile phases in the barrier layer (oxide layer) [351]. FTIR data (Figure 7.3a) yielded a significant peak for the TO (Figure 7.3b) and Ad+TO (Figure 7.3d) groups of $\mathrm{TiO}_{2}$ at $832 \mathrm{~cm}^{-1}$. Deconvolution was used to produce the summations of peaks. The $\mathrm{TiO}_{2}$ peak at $832 \mathrm{~cm}^{-1}$ was presented as predominantly rutile $\left(830 \mathrm{~cm}^{-1}\right)$ for the TO group, and a summation of peaks at $830 \mathrm{~cm}^{-1}$ and $870 \mathrm{~cm}^{-1}$, indicating the presence of rutile and anatase respectively, in the Ad+TO group [352]. Additional peaks found in the $\mathrm{TO}$ group for oxides of the alloying metals were $\mathrm{VO}_{2}(715$ $\left.\mathrm{cm}^{-1}\right)$ [353], Al-O $\left(835 \mathrm{~cm}^{-1}\right)$ [354], and Ti-O $\left(940 \mathrm{~cm}^{-1}\right)$ [352]. The presence of anatase appears consistent with the anodization process at $60 \mathrm{~V}$ [352]. Both amorphous Ti oxide and anatase were present in the deconvolution of peaks from the Ad group (Figure 7.3c) at both $939 \mathrm{~cm}^{-1}$ and $769 \mathrm{~cm}^{-1}$, and $870 \mathrm{~cm}^{-1}$ respectively [352]. Additional peaks representing Ti-OH were found at $1136 \mathrm{~cm}^{-1}$ and $1007 \mathrm{~cm}^{-1}$ and $\mathrm{Al}-\mathrm{OH}$ at $979 \mathrm{~cm}^{-1}$ and $1072 \mathrm{~cm}^{-1}$ in the Ad group. Similarly, in the $\mathrm{Ad}+\mathrm{TO}$ group, Ti-OH presented at $1048 \mathrm{~cm}^{-1}$ [355], Al-OH at $980 \mathrm{~cm}^{-1}$ [356] and alumina at $935 \mathrm{~cm}^{-1}$ [352]. 


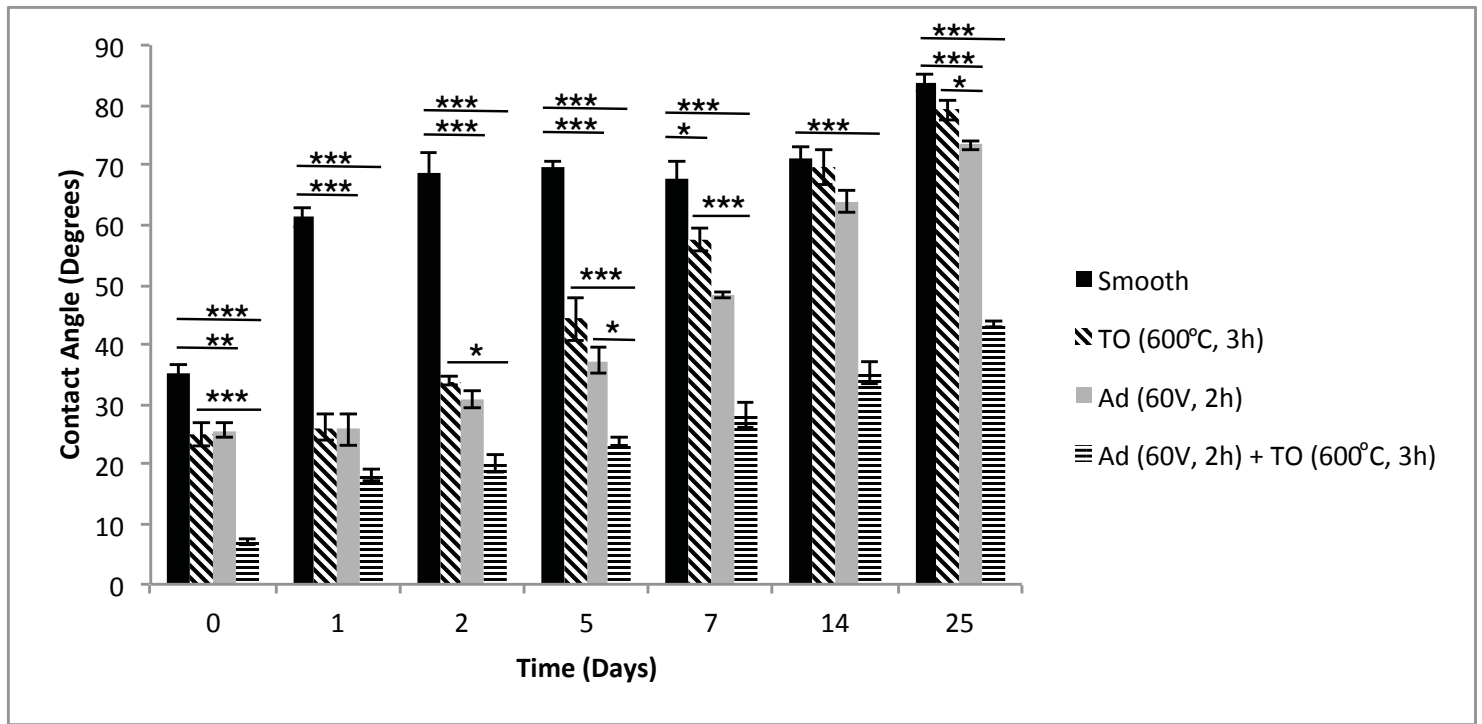

Figure 7.2 Water contact angle data for smooth, TO, Ad, and Ad+TO samples collected at days $0,1,2,5,7,14$, and 25 days of aging. (* $\mathrm{p}<0.05, * * \mathrm{p}<0.01, * * * \mathrm{p}<0.001)$. 


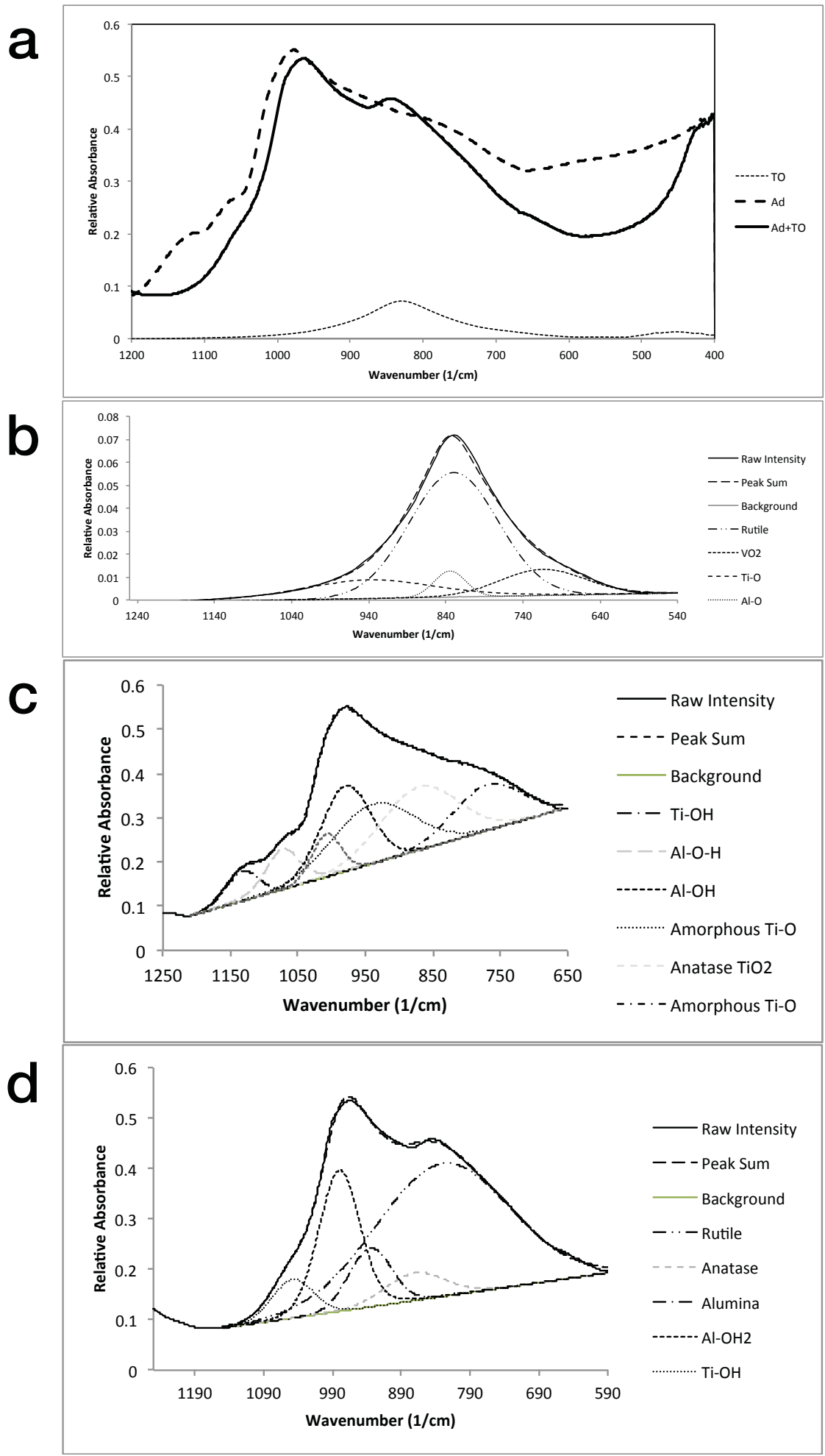

Figure 7.3 FTIR spectroscopy data for all samples (a), TO (b), Ad (c), and Ad+TO (d) samples measured against smooth background. 
(c) FESEM dimensional analysis: FESEM imaging showed evidence of similarly formed nanopores and TNTs in a honeycomb pattern indistinguishable for both Ad and Ad+TO sample groups (Figure 7.4). Images of the top of scratched TNTs (Figure 7.4a, Figure $7.4 \mathrm{~d}$ ) were used to determine the average inner diameter of the Ad and Ad+TO nanopores as $97.3 \pm 15 \mathrm{~nm}$, images of the bottom of scratched TNTs (Figure 7.4b, Figure 7.4e) were used to determine the average outer diameter of the Ad and Ad+TO TNTs as $167.8 \pm 15$ $\mathrm{nm}$, and side images of scratched TNTs (Figure 7.3c, Figure 7.3f) were used to determine the average length of the Ad and Ad+TO TNTs as $5.3 \pm 0.75 \mu \mathrm{m}$. It was also shown that the TO group did not consist of a nanotube surface (Figure $7.4 \mathrm{~g}$ ).

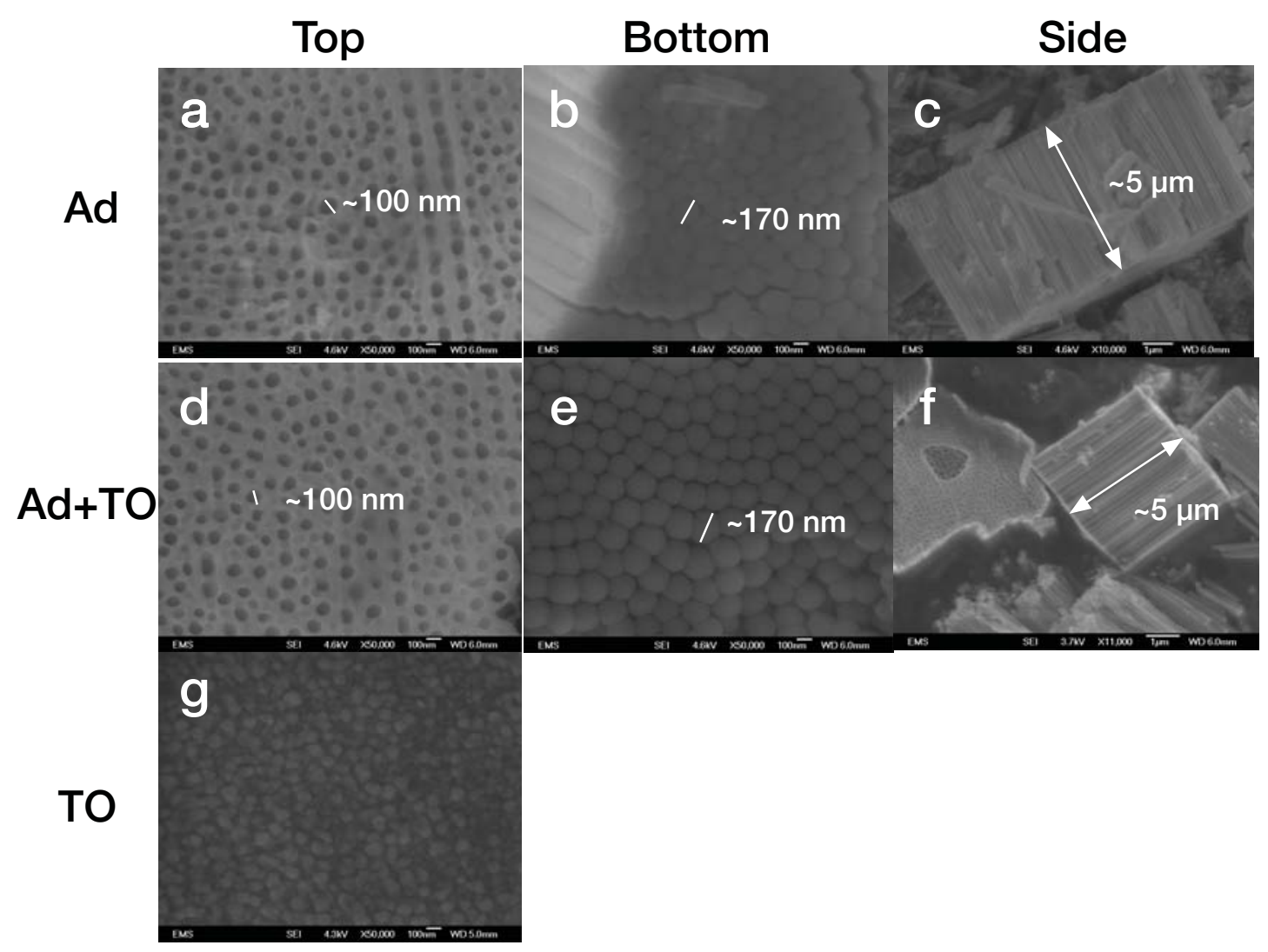

Figure 7.4 FESEM images for Ad TNTs top view (a), Ad TNTs bottom view (b), Ad TNTs side view (c), Ad+TO TNTs top view (d), Ad+TO TNTs bottom view (e), Ad+TO TNTs side view (f), and TO top view (g). Average nanotube diameters and lengths are displayed. 
(d) Surface area estimation by BET: BET surface area analysis also provided a quantitative approach to estimating the exposed surface area of anodized samples. While the smooth disc did not have enough total surface area to register, the anodized disc yielded a total surface area of $0.33 \mathrm{~m}^{2} / \mathrm{g}$. Using the sample mass of $0.67 \mathrm{~g}$ and the assumption of the surface area of the base and side of the disc being comparably negligible to that of the nanoporous surface, the estimated exposed surface area of the anodized sample was roughly $300 \mathrm{~cm}^{2}$ (Table 7.1). This value comes from the region of exposure to the electrolyte during corrosion testing being roughly $15 \%$ of the surface.

\section{Variation of electrochemical parameters}

(a) Evolution of free potential: Initially, OCP tests were conducted prior to EIS to determine the free potential of the surfaces. There are statistical differences between all sample groups $(\mathrm{p}<0.01)$ and can be seen in Figure 7.5. The OCP for the smooth, TO, Ad and $\mathrm{Ad}+\mathrm{TO}$ groups were $-525 \pm 44 \mathrm{mV}$ vs. SCE, $-330 \pm 25 \mathrm{mV}$ vs. SCE, $-204 \pm 48 \mathrm{mV}$ vs. SCE and $-46 \pm 27 \mathrm{mV}$ vs. SCE respectively (Table 7.2).

Table 7.1: BET surface area for anodized samples.

\begin{tabular}{|c|c|c|c|}
\hline Treatment & BET $\left(\mathbf{m}^{\mathbf{2}} / \mathbf{g}\right)$ & Correlation & BET Exposed Surface Area $\left.\mathbf{( c m}^{\mathbf{2}}\right)$ \\
\hline Anodized & 0.33 & 0.998 & $\sim 300$ \\
\hline
\end{tabular}


Table 7.2: OCP and cyclic polarization testing. OCP values, values of $\mathrm{E}_{\text {corr }}$ and $\mathrm{I}_{\text {corr }}$ determined from Tafel's method, and $I_{\text {pass }}$ values with standard deviations for the different treatment groups.

\begin{tabular}{|c|c|c|c|c|}
\hline Treatment & $\begin{array}{c}-\mathbf{E}_{\text {OCP }} \\
(\mathbf{V} \text { vs SCE) }\end{array}$ & $\begin{array}{c}-\mathbf{E}_{\text {corr }} \\
(\mathbf{V} \text { vs SCE) }\end{array}$ & $\begin{array}{c}\mathbf{I}_{\text {corr }} \\
\left(\boldsymbol{\mu A} / \mathbf{c m}^{2}\right)\end{array}$ & $\begin{array}{c}\mathbf{I}_{\text {pass }} \\
\left(\boldsymbol{\mu A} / \mathbf{c m}^{2}\right)\end{array}$ \\
\hline Smooth & $525(44)$ & $875(17)$ & $2.2 \times 10^{-1}(0.36)$ & $12.6 \times 10^{0}(0)$ \\
\hline TO & $330(25)$ & $678(17)$ & $3.5 \times 10^{-1}(0.23)$ & $2.1 \times 10^{0}(0.32)$ \\
\hline Anodized & $204(48)$ & $776(12)$ & $2.5 \times 10^{-3}(0.7)$ & $2.8 \times 10^{-2}(0.1)$ \\
\hline Anodized + TO & $46(27)$ & $317(57)$ & $6.0 \times 10^{-5}(5.6)$ & $8.6 \times 10^{-2}(1.5)$ \\
\hline
\end{tabular}

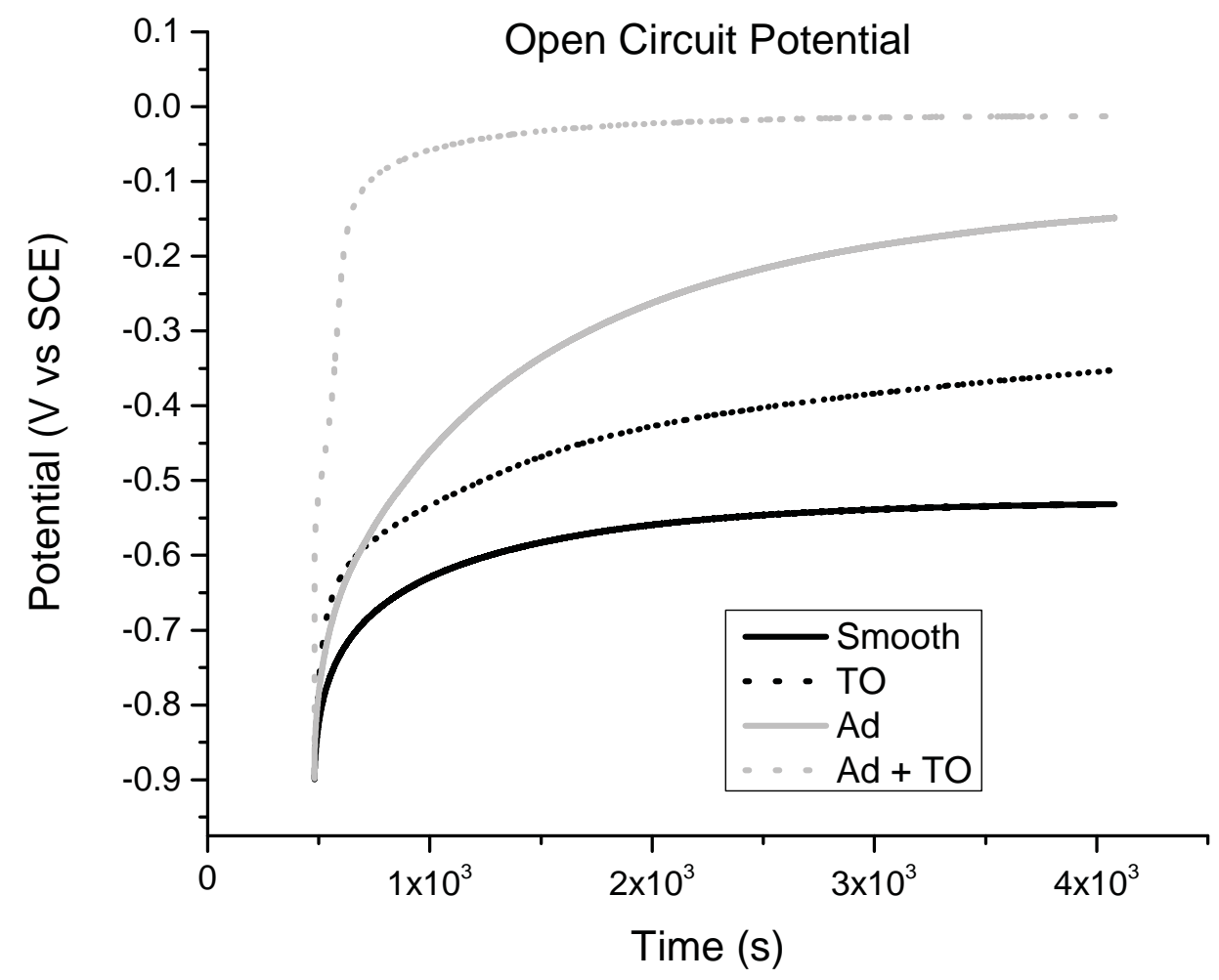

Figure 7.5 OCP curves for smooth, TO, Ad, and Ad+TO samples. 
(b) Changes in corrosion potential, corrosion current, and passivation behavior: The Tafel's slope method was to calculate $\mathrm{E}_{\text {corr }}$ and $\mathrm{I}_{\text {corr }}$ for the various sample groups. Figure 7.6 corresponds to the incorporated BET surface area estimation. Statistical analysis yielded significant differences between $\mathrm{Ad}+\mathrm{TO}$ and all other treatment group means $(\mathrm{p}<0.001)$. The TO group also showed a significant shift in the noble (positive) direction compared to the Ad group $(\mathrm{p}=0.009)$ and smooth $(\mathrm{p}<0.001)$. $\mathrm{E}_{\text {corr }}$ among the sample groups was, from greatest to smallest (or smallest to greatest in magnitude), $-317 \pm 57 \mathrm{mV}$ vs. SCE for $\mathrm{Ad}+\mathrm{TO},-678 \pm 17 \mathrm{mV}$ vs. SCE for TO, $-776 \pm 12 \mathrm{mV}$ vs. SCE for Ad, and $875 \pm 17 \mathrm{mV}$ vs. SCE for smooth (Table 7.2). Similar to the incorporation of estimated surface area for samples with TNTs for EIS analysis, BET estimated surface area was included in the calculation of the corrosion current density, which serves an indicator of the corrosion rate. $I_{\text {corr }}$ among the sample groups was, from smallest to greatest, was $6.0 \times 10^{-5} \mu \mathrm{A} / \mathrm{cm}^{2}$ for $\mathrm{Ad}+\mathrm{TO}, 2.5 \times 10^{-3} \mu \mathrm{A} / \mathrm{cm}^{2}$ for $\mathrm{Ad}, 3.5 \times 10^{-1} \mu \mathrm{A} / \mathrm{cm}^{2}$ for $\mathrm{TO}$, and $2.2 \times 10^{-1} \mu \mathrm{A} / \mathrm{cm}^{2}$ for smooth (Table 7.2). $\mathrm{I}_{\text {corr }}$ for $\mathrm{Ad}+\mathrm{TO}$ and Ad treatments were significantly lower than TO and smooth $(p<0.001)$. The forward scan of the cyclic polarization test was used to estimate the passivation current density ( $\left.\mathrm{I}_{\text {pass }}\right)$ at $1.5 \mathrm{~V}$ vs. SCE (Figure 7.7) and to analyze the passivation behavior. Estimated TNT surface area was incorporated in the same method as for $\mathrm{I}_{\text {corr }}$ calculations. From smallest to greatest, $\mathrm{I}_{\mathrm{pass}}$ for the different sample groups was determined to be $\mu \mathrm{A} / \mathrm{cm}^{2} 2.8 \times 10^{-2} \mu \mathrm{A} / \mathrm{cm}^{2}$ for Ad, $8.6 \times 10^{-2} \mu \mathrm{A} / \mathrm{cm}^{2}$ for $\mathrm{Ad}+\mathrm{TO}, 2.1 \mu \mathrm{A} / \mathrm{cm}^{2}$ for $\mathrm{TO}$, and $12.6 \mu \mathrm{A} / \mathrm{cm}^{2}$ for smooth (Table 7.2). $\mathrm{I}_{\text {pass }}$ for $\mathrm{Ad}+\mathrm{TO}$ and Ad treatments were significantly lower than $\mathrm{TO}$ and smooth $(\mathrm{p}<0.001)$. 
a

Passive Layer

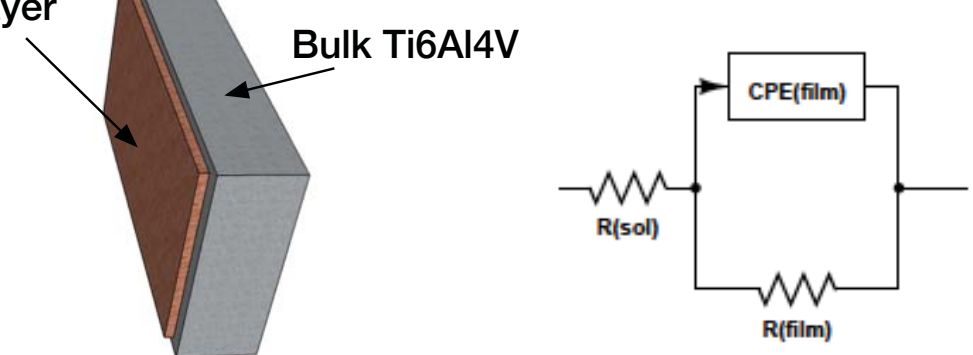

b
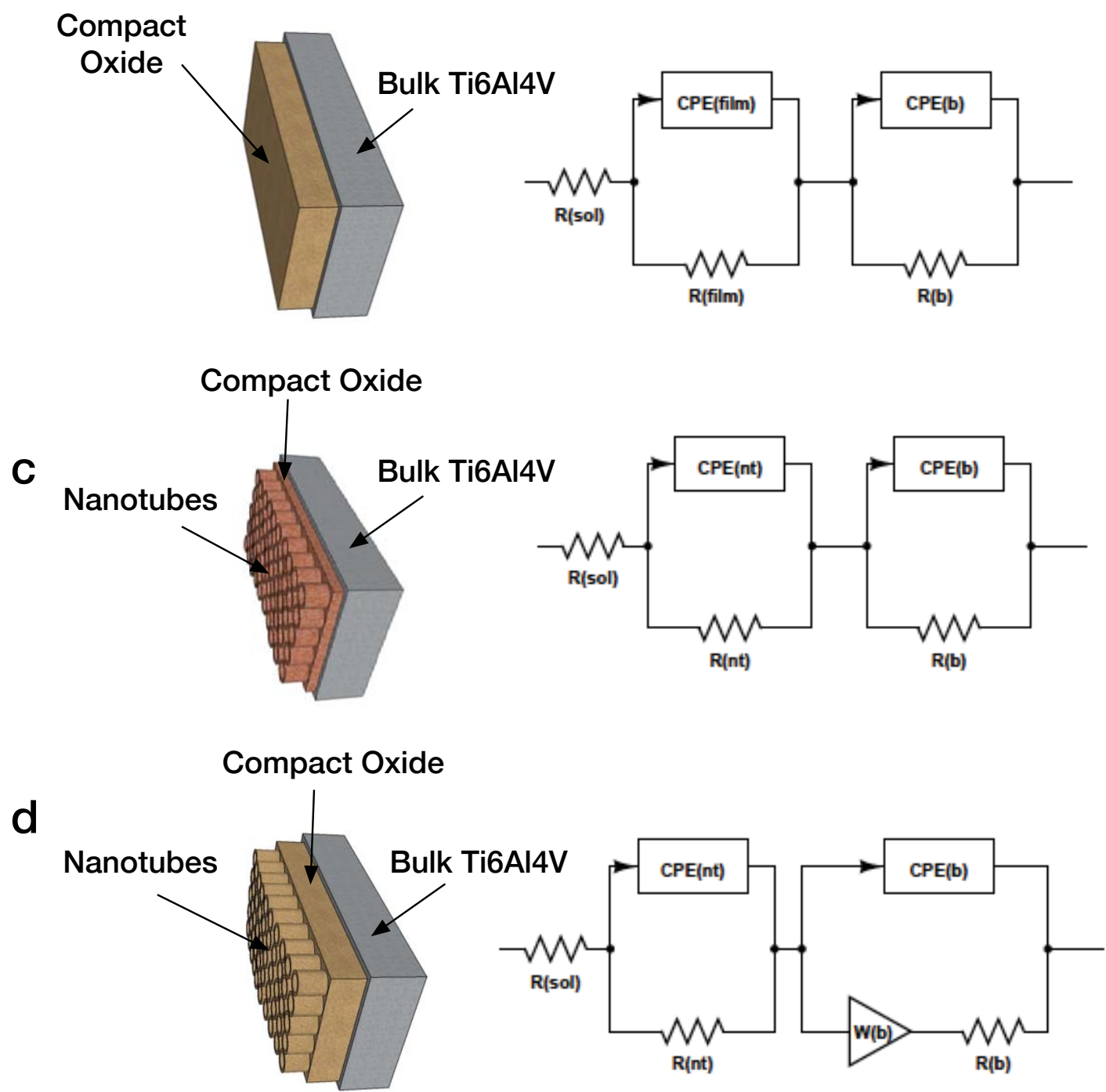

Figure 7.6 Cyclic polarization curves for smooth, TO, Ad, and Ad+TO samples. 


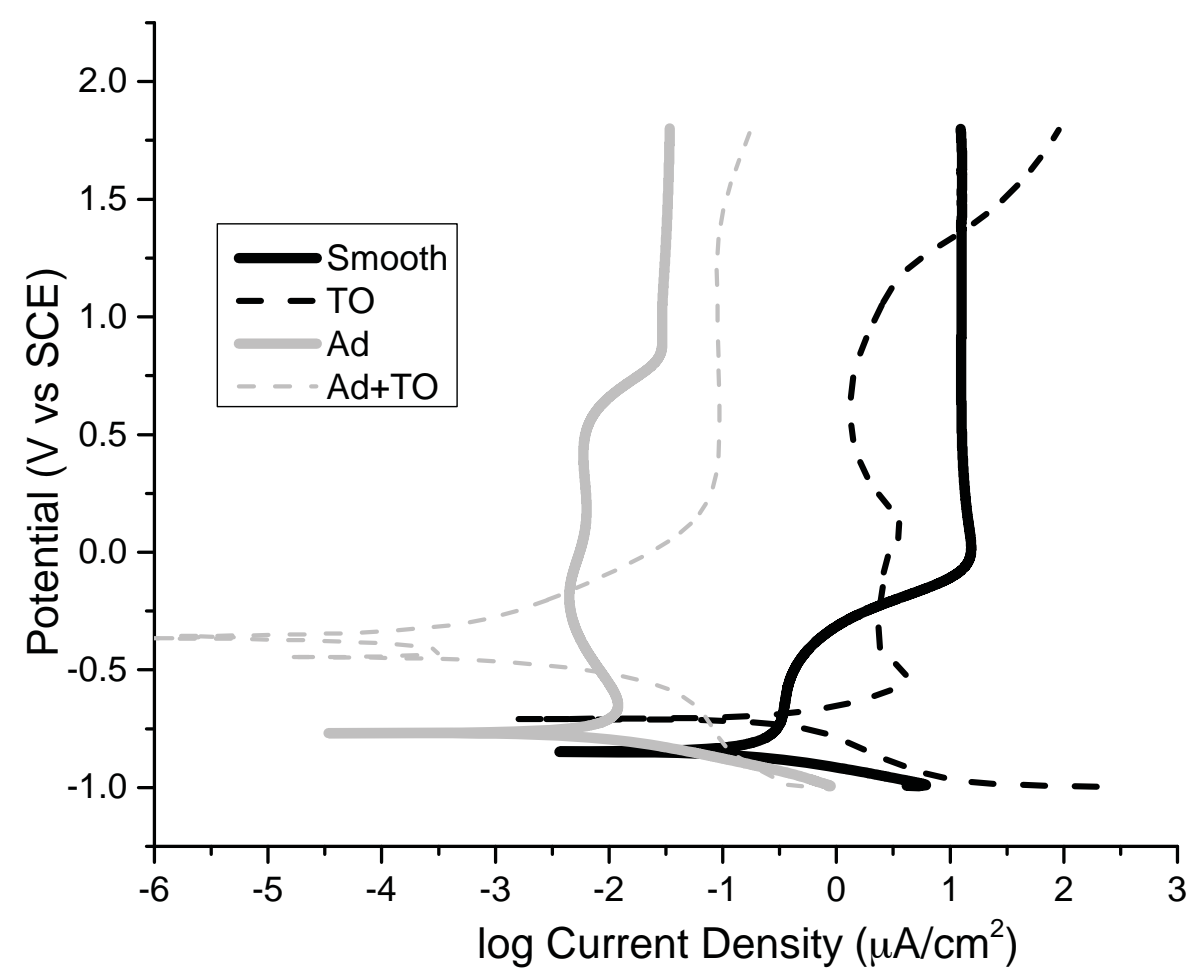

Figure 7.7 Equivalent circuits for smooth (a), TO (b), $\mathrm{Ad}(\mathrm{b})$, and $\mathrm{Ad}+\mathrm{TO}$ (c) used to measure frequency responses of EIS. Individual circuit components were used to estimate $\mathrm{R}_{\text {eff }}$ and $\mathrm{CPE}_{\text {eff, }}$ and circuit model fitting was used to estimate $\mathrm{R}_{\mathrm{p}}$.

(c) EIS data and equivalent circuits: Equivalent circuit models for smooth (Figure 7.6a), TO and Ad (Figure 7.6b), and Ad+TO (Figure 7.6c) were used to analyze EIS data (Table 7.3), and thereby estimate the effective circuit resistance ( $\left.R_{\text {eff }}\right)$ and the effective circuit capacitance in the form of a constant phase element ( $\left.\mathrm{CPE}_{\text {eff }}\right)$ (Table 7.4). $\mathrm{R}_{\text {eff }}$ and $\mathrm{CPE}_{\text {eff }}$ were determined by Equation 1 and Equation 2 respectively. Either $\mathrm{R}_{\text {film }}$ or $\mathrm{R}_{\mathrm{np}}$, and $\mathrm{CPE}_{\text {film }}$ or $\mathrm{CPE}_{\mathrm{np}}$ were used depending on the circuit model, and are further explained. 
$R_{e f f}=R_{b}+R_{f i l m / n p}$

$C P E_{\text {eff }}=\left(\frac{1}{C P E_{b}}+\frac{1}{C P E_{\text {film } / n p}}\right)^{-1}$

The circuit model for the smooth group consisted of a modified Randle's circuit with electrolyte solution resistance $\left(\mathrm{R}_{\text {sol }}\right)$, film resistance $\left(\mathrm{R}_{\text {film }}\right)$ and capacitance $\left(\mathrm{CPE}_{\text {film }}\right)$, the TO group consisted of electrolyte solution resistance $\left(\mathrm{R}_{\text {sol }}\right)$, barrier resistance $\left(\mathrm{R}_{\mathrm{b}}\right)$ and capacitance $\left(\mathrm{CPE}_{\mathrm{b}}\right)$ in series with film resistance $\left(\mathrm{R}_{\text {film }}\right)$ and capacitance $\left(\mathrm{CPE}_{\text {film }}\right)$, the Ad group consisted of solution resistance $\left(\mathrm{R}_{\text {sol }}\right)$, barrier resistance $\left(\mathrm{R}_{\mathrm{b}}\right)$ and capacitance $\left(\mathrm{CPE}_{\mathrm{b}}\right)$ in series with the nanoporous layer resistance $\left(\mathrm{R}_{\mathrm{np}}\right)$ and capacitance $\left(\mathrm{CPE}_{\mathrm{np}}\right)$ and the $\mathrm{Ad}+\mathrm{TO}$ group consisted of electrolyte solution resistance $\left(\mathrm{R}_{\mathrm{sol}}\right)$, barrier resistance $\left(\mathrm{R}_{\mathrm{b}}\right)$ and capacitance $\left(\mathrm{CPE}_{\mathrm{b}}\right)$ in series with a Warburg diffusion element $\left(\mathrm{W}_{\mathrm{b}}\right)$ and the nanoporous layer resistance $\left(\mathrm{R}_{\mathrm{np}}\right)$ and capacitance $\left(\mathrm{CPE}_{\mathrm{np}}\right)$. CPE elements were used rather than ideal capacitors due to evidence of time dependence and non-ideal capacitor behavior in the Nyquist and Bode plots (Figure 7.8, Figure 7.9). Additionally, due to the presence of serum proteins, properties of interface adsorption elements may be included in the $\mathrm{TO}, \mathrm{Ad}$, and $\mathrm{Ad}+\mathrm{TO}$ group layers due to their increased surface roughness and previous use in TNT corrosion studies with serum proteins [349]. It has been shown that surface roughness, even compared to surface wettability, is a strong determinant of protein adsorption [357]. Table 7.3 displays all circuit element values as well as $\mathrm{X}^{2}$ values determining the goodness of fit. While the total effective circuit resistance $\left(\mathrm{R}_{\mathrm{eff}}\right)$ was greatest for the smooth group at $7.1 \mathrm{M} \Omega$, it is important to consider the resistance and capacitance values with the associated surface area. For the $\mathrm{Ad}$ and $\mathrm{Ad}+\mathrm{TO}$ groups, the value obtained from BET surface area analysis was used. The order of greatest to smallest $\mathrm{R}_{\text {eff }}$ was $\mathrm{Ad}\left(1.2 \times 10^{8} \Omega . \mathrm{cm}^{2}\right)$, smooth $\left(2.7 \times 10^{6} \Omega . \mathrm{cm}^{2}\right)$, Ad+TO $\left(2.3 \times 10^{6} \Omega . \mathrm{cm}^{2}\right)$, and TO $\left(7.6 \times 10^{5} \Omega . \mathrm{cm}^{2}\right)$, with a significant difference between Ad and other treatment groups $(\mathrm{p}=0.017)$. 
Table 7.3: EIS modeling parameters obtained using the equivalent circuits shown in Figure 7.7 for EIS diagrams presented in Figure 7.8 and Figure 7.9 with standard deviations.

\begin{tabular}{|c|c|c|c|c|c|c|c|c|c|}
\hline Treatment & $\begin{array}{l}\mathbf{R}_{\text {sol }} \\
(\mathbf{\Omega})\end{array}$ & $\begin{array}{c}\mathrm{CPE}_{\mathrm{b}} \\
\left(\mathrm{F} . \mathrm{s}^{\mathrm{a}-1}\right)\end{array}$ & $\mathbf{a}_{\mathbf{b}}$ & $\begin{array}{l}\mathbf{R}_{\mathrm{b}} \\
(\mathbf{\Omega})\end{array}$ & $\begin{array}{c}W_{b} \\
\left(\Omega . s^{-1 / 2}\right)\end{array}$ & $\begin{array}{c}\mathrm{CPE}_{\text {film/np }} \\
\left(\mathrm{F} \cdot \mathrm{s}^{\mathrm{a}-1}\right)\end{array}$ & $a_{\text {film} / n p}$ & $\begin{array}{c}R_{\text {film/np }} \\
(\Omega)\end{array}$ & $\mathbf{X}^{2}$ \\
\hline Smooth & $\begin{array}{c}68 \\
(11)\end{array}$ & & & & & $\begin{array}{c}1.7 \times 10^{-5} \\
(0.17)\end{array}$ & 0.98 & $\begin{array}{c}7.1 \times 10^{6} \\
(2.2)\end{array}$ & $6.7 \times 10^{-3}$ \\
\hline $\mathrm{TO}$ & $\begin{array}{c}54 \\
(11)\end{array}$ & $\begin{array}{l}6.4 \times 10^{-} \\
5(1.3)\end{array}$ & 0.67 & $\begin{array}{c}2.0 \times 10^{6} \\
(1.9)\end{array}$ & & $\begin{array}{c}5.6 \times 10^{-5} \\
(1.5)\end{array}$ & 0.63 & $\begin{array}{c}3.1 \times 10^{3} \\
(0.31)\end{array}$ & $3.7 \times 10^{-3}$ \\
\hline$\overline{\mathrm{Ad}}$ & $\begin{array}{l}77 \\
(3)\end{array}$ & $\begin{array}{l}4.0 \times 10^{-} \\
{ }^{5}(1.8)\end{array}$ & 0.88 & $\begin{array}{c}3.8 \times 10^{5} \\
(2.0)\end{array}$ & & $\begin{array}{c}2.9 \times 10^{-5} \\
(0.52)\end{array}$ & 0.78 & $\begin{array}{c}2.7 \times 10^{4} \\
(4.5)\end{array}$ & $1.0 \times 10^{-2}$ \\
\hline $\mathrm{Ad}+\mathrm{TO}$ & $\begin{array}{c}24 \\
(12)\end{array}$ & $\begin{array}{l}1.9 \times 10^{-} \\
5(1.2)\end{array}$ & 0.66 & $\begin{array}{c}4.9 \times 10^{3} \\
(2.7)\end{array}$ & $\begin{array}{c}1.16 \times 10^{3} \\
(0.16)\end{array}$ & $\begin{array}{c}1.5 \times 10^{-5} \\
(0.7)\end{array}$ & 0.72 & $\begin{array}{c}2.8 \times 10^{3} \\
(2.6)\end{array}$ & $1.7 \times 10^{-3}$ \\
\hline
\end{tabular}

Table 7.4: Effective resistance and capacitance calculations and $\mathrm{R}_{\mathrm{p}}$. Values calculated for $\mathrm{R}_{\text {eff }}$ and $\mathrm{CPE}_{\text {eff }}$ from equivalent circuit modeling as well as $\mathrm{R}_{\mathrm{p}}$ values determined from Figure 7.9 with standard deviations.

\begin{tabular}{|c|c|c|c|}
\hline Treatment & $\begin{array}{c}\mathbf{R}_{\mathbf{p}} \\
\left(\mathbf{\Omega . c m}^{2}\right)\end{array}$ & $\begin{array}{c}\mathbf{R}_{\text {eff }} \\
\left(\mathbf{\Omega . c m}^{2}\right)\end{array}$ & $\begin{array}{c}\mathbf{C P E}_{\text {eff }} \\
\left(\mathbf{F . s .}^{-2-1} / \mathbf{c m}^{2}\right)\end{array}$ \\
\hline Smooth & $5.5 \times 10^{5}(0.69)$ & $2.7 \times 10^{6}(0.86)$ & $4.3 \times 10^{-5}(0.44)$ \\
\hline TO & $6.4 \times 10^{4}(1.2)$ & $7.6 \times 10^{5}(7.4)$ & $7.7 \times 10^{-5}(1.5)$ \\
\hline Ad & $1.0 \times 10^{8}(0.18)$ & $1.2 \times 10^{8}(0.74)$ & $5.4 \times 10^{-8}(1.3)$ \\
\hline Ad+TO & $5.2 \times 10^{6}(1.0)$ & $2.3 \times 10^{6}(0.56)$ & $6.3 \times 10^{-8}(3.9)$ \\
\hline
\end{tabular}



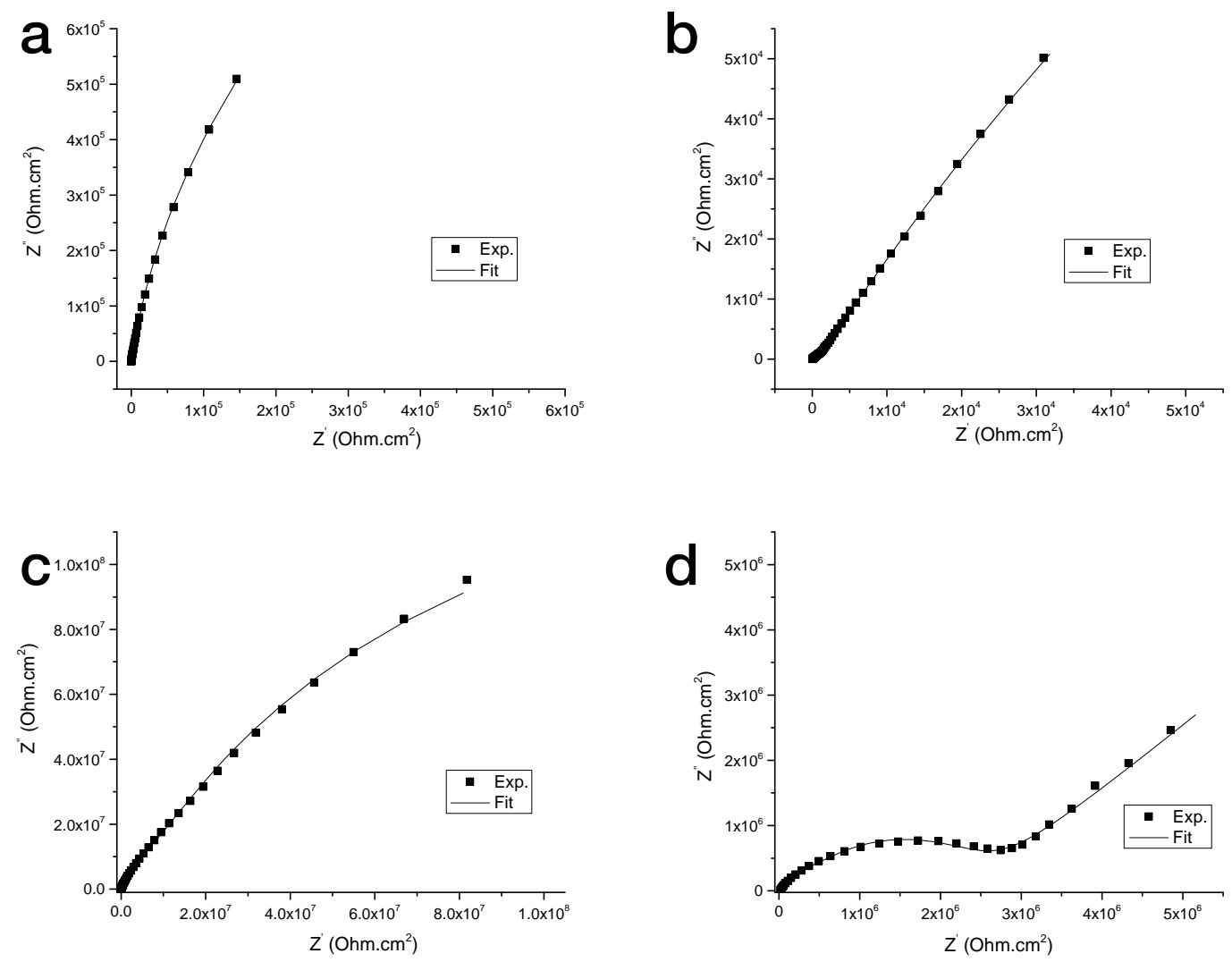

Figure 7.8 Nyquist plots exhibiting experimental and equivalent circuit modeling for smooth (a), TO (b), Ad (c), and Ad+TO (d) surfaces. 

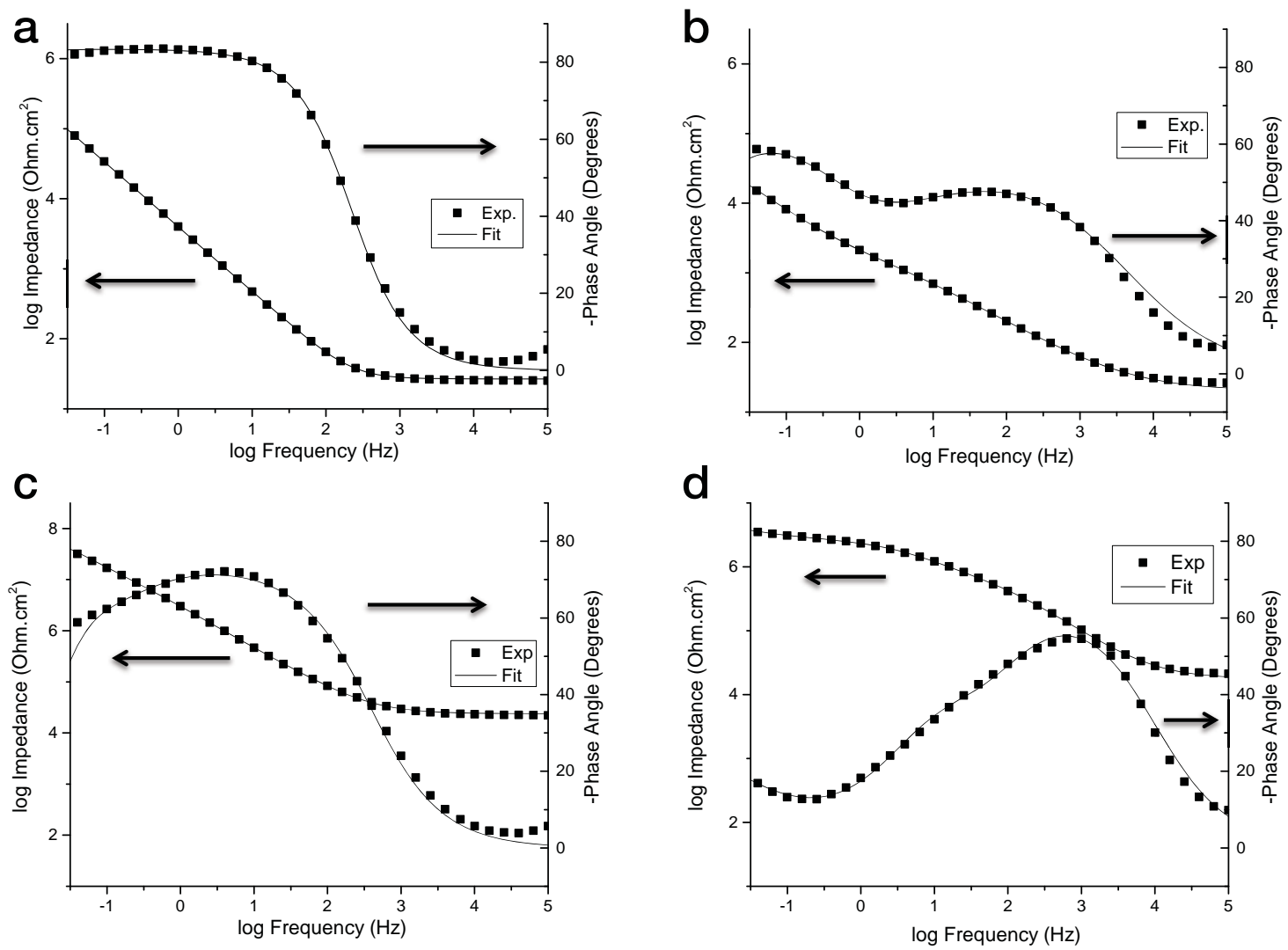

Figure 7.9 Impedance (a) and phase angle (b) Bode plots exhibiting experimental and equivalent circuit modeling for sample groups with BET surface area analysis for anodized samples.

Regarding the effective circuit $\mathrm{CPE}\left(\mathrm{CPE}_{\text {eff }}\right)$, the order from greatest to smallest was smooth $\left(4.3 \times 10^{-5} \mathrm{~F} / \mathrm{cm}^{2}\right)$, TO $\left(7.7 \times 10^{-5} \mathrm{~F} / \mathrm{cm}^{2}\right)$, $\mathrm{Ad}+\mathrm{TO}\left(6.3 \times 10^{-8} \mathrm{~F} / \mathrm{cm}^{2}\right)$, and $\mathrm{Ad}$ $\left(5.4 \times 10^{-8} \mathrm{~F} / \mathrm{cm}^{2}\right)$, with significant differences between both $\mathrm{Ad}+\mathrm{TO}$ and Ad treatments compared to smooth and TO $(\mathrm{p}<0.001)$. Ad+TO and Ad effective capacitance were not significantly different $(\mathrm{p}>0.05)$. Additionally, the $\mathrm{Ad}+\mathrm{TO}$ equivalent circuit was fitted with a Warburg diffusion element $\left(\mathrm{W}_{\mathrm{b}}=1.16 \times 10^{3}\right)$, indicating diffusion limited charge transfer. Nyquist and Bode plots (Figure 7.8, Figure 7.9) display experimental and equivalent circuit fitting. $R_{\text {eff }}$ was calculated as an estimation of the equivalent resistance of each circuit. This value is often related to the polarization resistance, however, this made for a difficult comparison due to the varying components of each circuit, for 
example, the inclusion of a Warburg diffusion element in the $\mathrm{Ad}+\mathrm{TO}$ group as indicated by the diffusion tail in the Nyquist plot (Figure 7.8d). It is known that $R_{p}$ can also be estimated by using the $y$-intercept of the Bode resistance and subtracting $R_{\text {sol }}$ [358], thus Bode plots were also used to determine the polarization resistance $\left(R_{p}\right)$ of the sample electrodes (Table 7.4). The order of sample groups from largest to smallest $R_{p}$ value was $\mathrm{Ad}>\mathrm{Ad}+\mathrm{TO}>$ smooth $>\mathrm{TO}$, with significant differences between the Ad treatment and all other treatment groups $(\mathrm{p}<0.001)$. The $\mathrm{R}_{\mathrm{p}}$ values determined from Bode plot analysis appear to be more consistent with further discussed indicators of superior corrosion resistance of anodized samples, and may provide a better understanding of the polarization resistance compared to $\mathrm{R}_{\text {eff. }} \mathrm{C}_{\text {eff }}$ results also appear consistent with Bode plot analysis. Smooth samples (Figure 7.9a) yielded high capacitive behavior within low frequencies $(<10 \mathrm{~Hz})$ with a phase angle $>80^{\circ}$ and low capacitive behavior within high frequencies $(>1000 \mathrm{~Hz})$. A similar curve shape can be seen with the Ad samples (Figure $7.9 \mathrm{c})$, indicating the similarities among amorphous oxide layers, however the phase angle magnitude at lower frequencies is smaller than that of the smooth group, meaning lower capacitive behavior. The TO samples (Figure 7.9b) also showed higher capacitive behavior within low frequencies, but in lower magnitude (phase angle near $60^{\circ}$ ) than both smooth and Ad samples. Conversely, the Ad+TO group (Figure 7.9d) yielded higher capacitive behavior in higher frequencies, with the lowest magnitude phase angle of all groups. There appeared to be capacitive behavior within all frequency ranges of the $\mathrm{Ad}+\mathrm{TO}$ group, with a range of phase angles from $17^{\circ}$ to $56^{\circ}$. The graphical analysis is consistent with $\mathrm{CPE}_{\text {eff }}$ being lowest within the $\mathrm{Ad}+\mathrm{TO}$ group, and supports its high $\mathrm{R}_{\mathrm{p}}$ due to the thick barrier oxide layer.

\subsection{Discussion}

\section{Understanding the surface properties}

Enhanced surface wettability of all treated groups as received is explained by both increased surface roughness and anatase/rutile crystalline phases for thermally oxidized groups, and by increased surface roughness and presence of hydroxyl groups $\left(\mathrm{Ti}(\mathrm{OH})_{4}\right)$ 
from the electrochemical anodization process [338], verified by FTIR analysis (Figure 7.3). For the Ad group, aging led to increased WCA due to replacement of $\operatorname{Ti}(\mathrm{OH})_{4}$ by $\mathrm{TiO}_{2}$ [338], and it appears that annealing the nanoporous substrate increased their stability and slowed the process of hydroxyl group replacement. These results indicate the $\mathrm{Ad}+\mathrm{TO}$ samples superior wettability and conduciveness to cell culture and in vivo environments. FESEM revealed that much of the surface was covered with a nanoporous layer on top of the TNT layer. This can be attributed to remnants of the initial barrier layer during anodization [359]. Nanopore and nanotube dimensions were consistent with TNT literature regarding voltage, anodization solution, and duration [333]. The determination of the surface area exposed to the electrolyte proved to be challenging, yet it is an integral part of analyzing EIS and potentiodynamic data, and often is not discussed in nanotube corrosion literature. Thus, it was useful to provide quantitative measurements to estimate resistance, capacitance and current densities across the substrate surface-solution interface. The BET method is a sensitive and accurate method for measuring surface area, and has been used to estimate titania nanotube surface area (in some cases as much as 30 $\mathrm{m}^{2} / \mathrm{g}$ ) [333], however with the treated alloyed substrates, the surfaces are not uniform. That is, the treatments (specifically anodization) only occur on one face of the disc. However, it is a reasonable assumption that most of the surface area measured by the BET method can be attributed to the nanoporous surface. Additionally, qualitative comparisons of inadequate total surface area for BET analysis with smooth samples as well as average nanotube dimensions suggest vastly increased surface area in anodized samples ( $\mathrm{Ad}$ and $\mathrm{Ad}+\mathrm{TO}$ ) compared to smooth and TO samples.

\section{Variation in electrochemical potential and kinetics}

The treatment OCP values provide an indication of the electrode's tendency corrode in reaching steady state redox reactions, thus it can be concluded that the $\mathrm{Ad}+\mathrm{TO}$ group displayed the lowest corrosion tendency due to presenting the most positive potential (the smallest difference in potential) with respect to the reference electrode. Regarding potentiodynamic testing, analysis of the anodized treatment groups, that is, Ad 
and $\mathrm{Ad}+\mathrm{TO}$, showed excellent corrosion resistance, with the $\mathrm{Ad}+\mathrm{TO}$ showing the greatest resistance to corrosion, with regard to $\mathrm{E}_{\text {corr }}$ and $\mathrm{I}_{\text {corr }}$ parameters. For the $\mathrm{Ad}+\mathrm{TO}$ group, there appeared to be two Tafel regions within the scan. The region in which the current density fell closest to $0 \mu \mathrm{A} / \mathrm{cm}^{2}$ was used to calculate $\mathrm{E}_{\text {corr }}$ and $\mathrm{I}_{\text {corr. }}$. The double region may have occurred due to the presence of polycrystalline TNTs or due to excess hydrogen evolution in the cathodic region. Similar to the OCP testing, the extrapolated $\mathrm{E}_{\text {corr }}$ using the Tafel method serves as an indication of the working electrode's tendency to corrode by displaying the potential at which the anodic current begins to exceed the cathodic. A clear shift of OCP and $\mathrm{E}_{\text {corr }}$ in the noble direction for TNT layer samples was observed, with the greatest being for the $\mathrm{Ad}+\mathrm{TO}$ group, indicating a decreased tendency to corrode in the cell media electrolyte. This analysis is consistent with previous studies regarding thermally oxidized TNTs, in which the sample material and method of anodization differs from that of this study [348,349,360]. Compared to OCP analysis, the $\mathrm{E}_{\text {corr }}$ analysis showed that $\mathrm{TO}$ had a nobler $\mathrm{E}_{\text {corr }}$ than $\mathrm{Ad}$, and all were nobler than the smooth control. The results are indicative that the presence of a thick amorphous and anatase TNT layer provides more stability than the thin spontaneous amorphous oxide layer. It further suggests that the thermal oxidation forming predominantly rutile crystalline structure in the non-anodized group provides a more stable crystalline phase at $\mathrm{E}_{\text {corr }}$. The heat treatment appears to be additive to the nanoporous and nanotubular layers compared to the spontaneous layer of the smooth control, and can explain the differences in $E_{\text {corr }}$ for the various treatment groups. Though both the $\mathrm{Ad}$ and $\mathrm{Ad}+\mathrm{TO}$ had greater surface area exposure to the electrolyte, incorporating BET surface area, the corrosion rate $\left(\mathrm{I}_{\text {corr }}\right)$ appears to be roughly two orders of magnitude smaller in the $\mathrm{Ad}+\mathrm{TO}$ group than the Ad group, and four orders of magnitude smaller than the smooth group. Exact quantification between the groups is difficult to discern due to the surface area estimation method, however, significantly decreased $\mathrm{I}_{\text {corr }}$ due to surface area increases were shown. Low current densities are not only an indicator of slower local corrosion, but are also important in retaining cell viability at a substrate surface as high current densities have been shown to induce apoptosis and necrosis in cell culture [361]. The smooth samples showed little change in corrosion current density, appearing to display passivation until roughly $-0.3 \mathrm{~V}$ vs. SCE ( $575 \mathrm{mV}$ greater than $\mathrm{E}_{\text {corr }}$ ). The passive layer was then removed 
and corrosion occurred until stable passivation close to $0 \mathrm{~V}$ vs. SCE. The smooth sample also yielded the greatest $\mathrm{I}_{\text {pass. }}$. The TO samples showed unstable passivation beginning at roughly $-0.5 \mathrm{~V}$ vs. SCE $\left(178 \mathrm{mV}\right.$ greater than $\left.\mathrm{E}_{\text {corr }}\right)$ and most likely transpassivation dissolution at $1 \mathrm{~V}$ vs. $\mathrm{SCE}\left(1.3 \mathrm{~V}\right.$ greater than $\left.\mathrm{E}_{\mathrm{corr}}\right)$. This trend of depassivation can also be seen, and is more gradual, with the $\mathrm{Ad}+\mathrm{TO}$ samples.

\section{The effect of surface crystalline structure on corrosion (passivation)}

The corrosion/passivation trends may indicate the susceptibility of the different crystalline phases of the alloy oxides to mediate corrosion, passivation or depassivation during anodic polarization, in particular, the mix of anatase, rutile and alumina in the $\mathrm{Ad}+\mathrm{TO}$ group compared to predominantly rutile in the TO group. While the Ad+TO group did exhibit stable passivation at roughly $0.3 \mathrm{~V}$ vs. SCE (617 mV greater than $\left.\mathrm{E}_{\text {corr }}\right)$, and had $\mathrm{I}_{\mathrm{pass}}$ smaller than both smooth and TO samples, it did exhibit corrosive behavior during anodic regions slightly above $\mathrm{E}_{\text {corr }}$. The Ad samples exhibited unstable passivation in the scan immediately after $\mathrm{E}_{\text {corr, }}$ and began stable passivation at roughly $0.8 \mathrm{~V}$ vs. SCE (1.58 $\mathrm{V}$ greater than $\left.\mathrm{E}_{\text {corr }}\right)$. The instability reflects the composition of the nanotube layer as predominantly a mixture of anatase and amorphous $\mathrm{TiO}_{2}$. Depassivation of more unstable amorphous regions create smaller areas of increased corrosion or transpassivation dissolution until stable passivation is reached. The Ad samples also showed the lowest $\mathrm{I}_{\text {pass }}$. Compared to the $\mathrm{Ad}+\mathrm{TO}$ group, it was observed that passivation occurred in the Ad group immediately in anodic regions slightly higher than $\mathrm{E}_{\text {corr. }}$ Additionally, FESEM imaging showed deep corrosion crevices in the surface of the Ad+TO group (Figure 7.10b) compared to the Ad (Figure 7.10a) due to the anodic corrosion. The higher susceptibility to display corrosion current densities in the Ad+TO group under slight anodic polarization is most likely due to the increased presence of rutile crystalline structure. Increased grain boundaries may lead to increased corrosion, as well as increased resistance to charge transfer and diffusion-limited charge transfer observed in EIS. The interplay of diffusion limited charge transfer correlates to the 
corrosion and passivation mechanisms regarding the diffusion of metal ion and anionic oxygen species, and will be further discussed. 

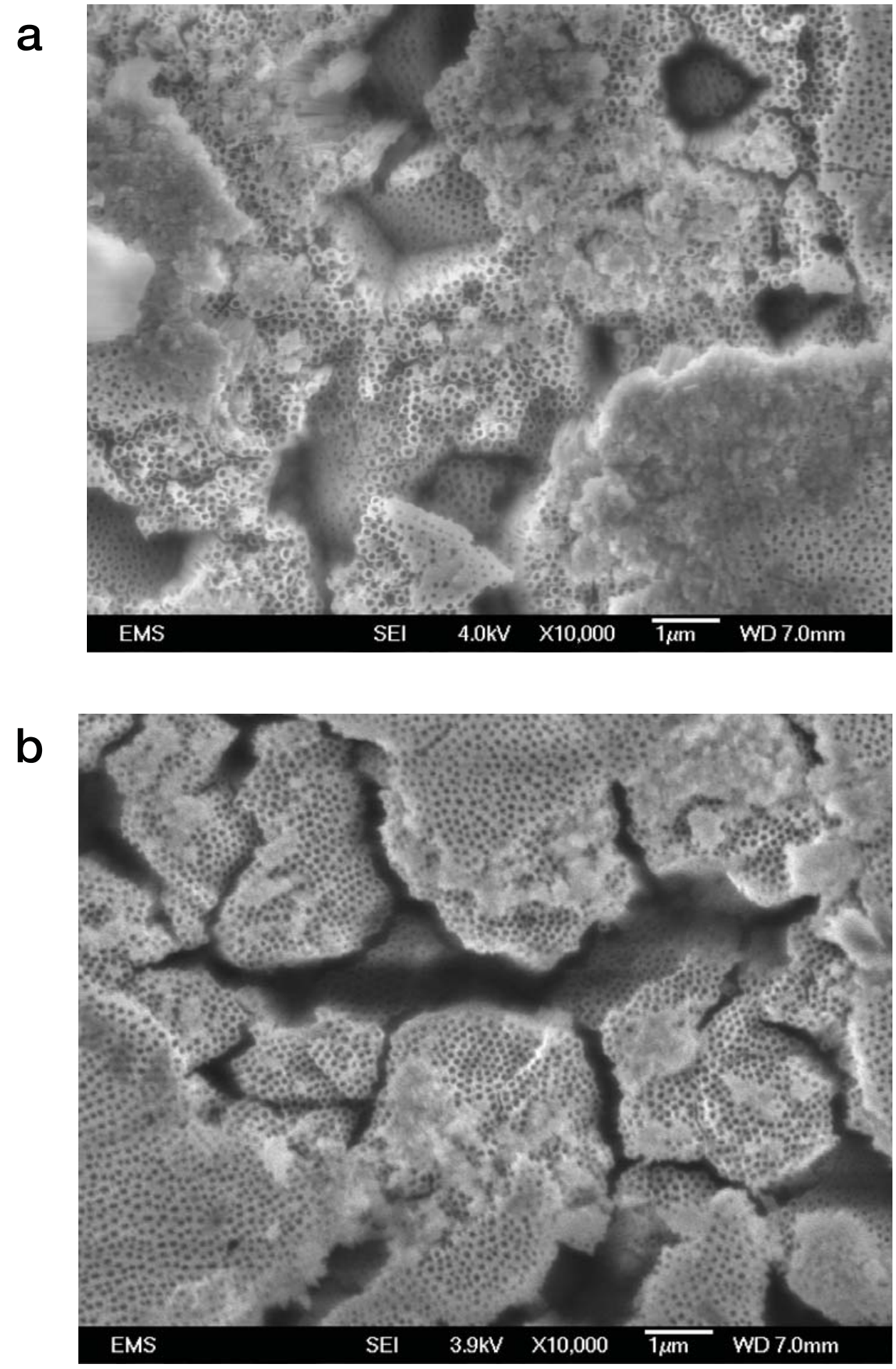

Figure 7.10 FESEM images of $\mathrm{Ad}(\mathrm{a})$ and $\mathrm{Ad}+\mathrm{TO}(\mathrm{b})$ after cyclic polarization. 


\section{Corrosion process and mechanisms}

In order to understand the corrosion and passivation behaviors during potentiodynamic polarization, it is necessary to understand the mechanisms and properties of the bulk and porous oxides of the different treatment groups. Figure 7.11 shows a simple proposed mechanism for corrosion and the release of metal ions in to the electrolyte solution coupled with passivation due to anodic polarization. Figure 7.11a and Figure $7.11 \mathrm{~b}$ are schematic representations of the smooth and TO groups, though they differ in thickness and crystalline composition of the oxide layer, while Figure 7.11c and Figure $7.11 \mathrm{~d}$ are schematic representations of the $\mathrm{Ad}$ and $\mathrm{Ad}+\mathrm{TO}$ groups, though they differ in thickness of the bulk oxide layer as well as crystalline composition of the bulk oxide and nanoporous layers. The difference between substrates that may impact the mechanisms are the thickness of the bulk oxide, presence of a porous nanotube layer, composition of both bulk and porous oxide layers, and wettability (due to the presence of serum proteins in the cell culture media). It has been shown that under anodic polarization, growth of the passive layer in Ti-6Al-4V occurs at the metal-oxide interface due to diffusion of anionic oxygen species through the passive layer, rather than at the oxide-solution interface due to metal ion diffusion as it does with $\mathrm{Ti}$ [362]. Thus, for passivation to occur, diffusion of oxygen species to the bulk metal is necessary with increasing anodic polarization, while diffusion of metal ion species through the oxide layer(s) to the solution interface is limited. In essence, anionic species are created at the solution front where their concentration remains constant, often from the splitting of water in aqueous solutions [363]. The increased wettability, surface roughness and nanotopography of $\mathrm{Ad}+\mathrm{TO}$ may lead to increased protein adsorption. This may be a contributing factor as to why the $\mathrm{Ad}+\mathrm{TO}$ group experienced stable passivation with a higher increase in current density in the potentiodynamic scan. The exact effect of protein prevalence is unknown, and is beyond the scope of this study, but the presence of a protein layer at the oxide-solution interface may inhibit the creation and diffusion of anionic oxygen species for passivation, while also inhibiting the dissolution of metal ion from the surface. The differences in crystalline structure of both compact and porous 
oxide layers also influence the corrosion/passivation kinetics. Polycrystalline materials offer different diffusion pathways for ion species, and understanding the movement of defects in the crystals can provide information as to the corrosion kinetics of polycrystalline Ti alloy oxides. In addition, nanotube layers offer diffusion pathways through nanotube pores as well as nanotube walls (Figure 7.11b). It has been reported that oxygen defects, both interstitial and vacancies, are faster diffusers in anatase compared to rutile [364], and that rutile offers greater resistance to charge transfer [333]. This may indicate the ability of anionic oxygen species to diffuse faster through anatase $\mathrm{TiO}_{2}$ than rutile, and would explain why the Ad group experienced almost immediate passivation, though unstable, compared to the $\mathrm{Ad}+\mathrm{TO}$ and $\mathrm{TO}$ treatments. There are clear benefits of having a mixture of anatase and rutile crystalline structures in TNTs and bulk oxide layers for wettability, the stability of the TNTs, lower tendency to corrode, and lower current densities. Further research can optimize the annealing temperature and duration, altering the anatase/rutile composition and thickness and thus improving the corrosion/passivation kinetics of the $\mathrm{Ad}+\mathrm{TO}$ treatment. 


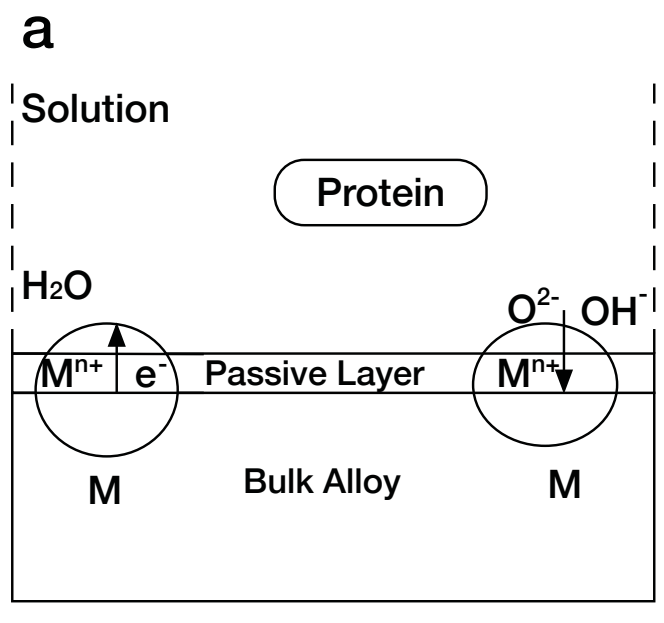

C
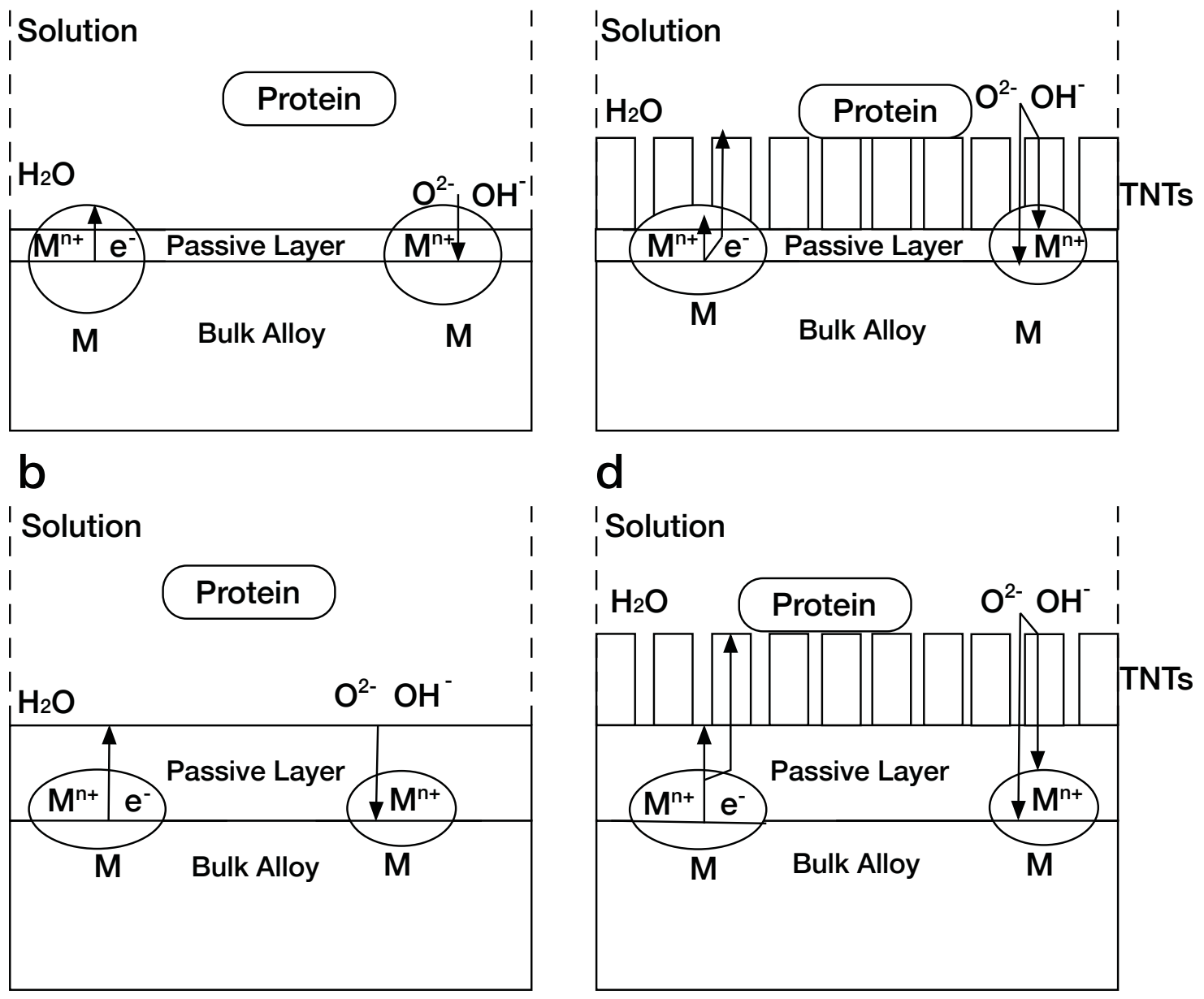

Figure 7.11 Proposed mechanism of corrosion/passivation for (a) smooth, (b) TO, (c) $\mathrm{Ad}$, and (d) Ad+TO substrates showing diffusion of metal ions $\left(\mathrm{M}^{\mathrm{n}+}\right)$ and anions, specifically $\mathrm{O}^{2-}$ and $\mathrm{OH}^{-}$, through the passive oxide layer. For each figure, the circle on the left indicates corrosion, while that on the right indicates passivation.

\subsection{Conclusion}

The following can be derived from this study:

- Electrochemical anodization was effective in producing anatase/amorphous $\mathrm{TiO}_{2}$ nanopores and nanotubes on Ti-6Al-4V substrates, while thermal oxidation $\left(600^{\circ}\right.$ C) effectively changed the crystalline structure to an anatase/rutile mixture. 
- The BET method showed the nanoporous surface had more than two orders of magnitude greater surface area than smooth surfaces.

- Annealed nanotubes not only enhanced surface roughness and wettability, but also enhanced corrosion resistance with respect to OCP, $E_{\text {corr }} I_{\text {corr }}, I_{\text {pass }}$ and capacitance and polarization resistance behavior. This was most likely due to increased thickness of the bulk oxide layer, and the stability and increased surface area of the polycrystalline nanotubular surface.

- The Ad+TO group had $\mathrm{E}_{\text {corr }}$ and $\mathrm{E}_{\mathrm{OCP}}$ shifts in the noble direction, as well as decreased $\mathrm{I}_{\text {corr }}$ and $\mathrm{I}_{\text {pass. }}$ Stable passivation was observed as well.

- Both anodized groups ( $\mathrm{Ad}$ and $\mathrm{Ad}+\mathrm{TO}$ ) exhibited high $\mathrm{R}_{\mathrm{p}}$, and EIS analysis indicated diffusion dependent limited charge transfer for $\mathrm{Ad}+\mathrm{TO}$.

- In slightly anodic regions compared to $\mathrm{E}_{\text {corr, }}$ anatase/amorphous nanotubes exhibited immediate passivation while annealed nanotubes exhibited corrosion and crevices in the surface.

- As per the findings, the treatment groups analyzed can be ordered from greatest corrosion resistance to least as $\mathrm{Ad}+\mathrm{TO}>\mathrm{Ad}>\mathrm{Smooth}>\mathrm{TO}$.

\section{Acknowledgements}

This work was supported by the NSF-CBET \# 1067424 and NSF-CMMI $\# 1160951$, and gratitude to the IBTN-USA members for their contributions. 


\section{Chapter 8}

\section{Discussion and Conclusions}

In this chapter major results of the research are overviewed briefly and discussed to reach the prominent conclusions of this study.

A review of literature shows titanium has recently attracted attention to be employed for dental and orthopedic applications. In comparison with other biomaterials, titanium provides some advantages including biocompatibility, corrosion resistance and suitable mechanical properties. Biocompatibility of titanium is attributed to presence of a native oxide layer that is formed on its surface. In order to further improve osseointegration at the surface, the outer oxide shell of titanium implants can be modified. In particular, production of $\mathrm{TiO} 2$ nanotubes over the surface of titanium by means of electrochemical anodization enhances attachment of cells to the surface and their proliferation and differentiation. Cellular behavior can be further improved by heat treating the anodized surface. In addition the nanotubes can act as nano-reservoirs of drugs to fabricate drug eluting implants.

When a biomaterial is placed in physiologic environment, the surrounding proteins are quickly adsorbed onto its surface and then these proteins intermediate attachment of cells to the underlying substrate. Considering that protein adsorption is higher to hydrophilic surfaces, hydrophilicity of the surface plays a key role in cellular attachment. Biocompatibility of the titanium surface can be attributed to its hydrophilicity. When titanium is anodized, hydrophilic nanotubes are formed that promote cellular response compared with non-anodized surface. Hydrophilicity of the anodized surface is further increased when it is thermally treated. In addition, cellular behavior is improved following heat treatment of the nanotubes. Our results show that

surface hydrophilicity can be optimized by controlling anodization and heat treatment conditions. It is shown that increasing anodization voltage leads to formation of larger 
nanotubes that provide a rougher surface with increased hydrophilicity. It is suggested that increasing anodization voltage results in increase of surface area and surface energy and consequently hydrophilicity is increased. In addition heat treatment shows an impact on surface characteristics. Our results show that non-heat treated samples lose their hydrophilicity sooner than those that were thermally treated at $300{ }^{\circ} \mathrm{C}$. The samples that were treated at $600{ }^{\circ} \mathrm{C}$ show the greatest ability to maintain their surface hydrophilicity over time. Surface hydrophilicity following anodization is associated to the existence of $\mathrm{OH}-$ groups in structure of $\mathrm{Ti}(\mathrm{OH}) 4$. Following exposure of anodized surface to air, the surface undergoes hydroxylation/dehydroxylation to eventually reach equilibrium. As $\mathrm{Ti}(\mathrm{OH}) 4$ transform into $\mathrm{TiO} 2$ to establish stability, the surface loses its hydrophilicity. Our results suggest that crystalline structure of the surface affects the hydroxylation/dehydroxylation equilibrium. In fact, anatase structure prolongs hydroxylation/dehydroxylation equilibrium in comparison with amorphous $\mathrm{TiO} 2$. The rutile crystalline structure shows the highest ability in retarding the establishment of hydroxylation/dehydroxylation equilibrium. It is conclude that shelf-life of implants can be extended if fabrication condition is optimized.

Not only formation of nanotubes increases surface hydrophilicity, but also they can be employed as drug reservoirs to provide local drug delivery. Compared with systemic delivery, local delivery of drug provides some benefits. For example, absorption of drug in digestive system is prevented and the drug can be delivered to avascular tissue that is left after surgery. However, burst release of drug from nanotubes reduces its efficiency and leads to denaturation of drug. To overcome this issue we have shown that optimizing dimension of nanotubes can be used to control the rate of drug release. Specifically, our results show that nanotubes with high aspect ratio prolong drug release. It is suggested that release of drug molecules is a function of diffusion. Therefore, either increasing the length of nanotubes or decreasing the diameter results in slower release of intercalated drug. Considering that increasing length of nanotubes does not affect cellular behavior, it is concluded that increasing aspect ratio of nanotubes by increasing their length can be used as a simple technique for controlling rate of drug release. 
Our results reveal that surface characteristics of titanium alloy are enhanced for biomedical applications through integrated anodization and annealing treatments. It is shown that desirable surface characteristics are provided by anodized samples after annealing. Water contact angle measurements of smooth, rough, anodized-smooth and anodized-rough surfaces before thermal oxidation reveals that anodized-smooth and anodized-rough substrates are super-hydrophilic while non-anodized smooth and rough surfaces show water contact angle of higher than $50^{\circ}$. All of the samples become superhydrophilic after thermal treatment but they show different characteristics after exposure to air. Although smooth and rough surfaces lose their hydrophilicity following aging, anodized-smooth and anodized-rough surfaces remain hydrophilic. This behavior can be explained by activity of $\mathrm{OH}^{-}$group on the surface. Considering that non-anodized surfaces possess less surface area and $\mathrm{OH}^{-}$groups, the aging period is enough for their complete dissociation. However, only a fraction of $\mathrm{OH}^{-}$group on the anodized surface is dissociated over time.

To verify a novel technique, production of nanotubes is performed after thermal oxidization of surface. During heat treatment various temperatures were applied to determine the role of crystalline structure of substrate on growth of nanotubes. Our results reveal that heat treatment prior to anodization can significantly influence obtained nanotubes in terms of length and surface morphology while chemical composition remains the same. It is known that oxidation at the interface of electrolyte and titanium surface results in formation of a barrier oxide layer. Following oxidation, $\mathrm{H}^{+}$ion is produced and accumulated which results in attraction of $\mathrm{F}^{-}$ions. Eventually, $\mathrm{F}^{-}$ions dissolve the barrier oxide layer and nanotubular structure is formed. Our data suggest that due to quicker formation of barrier oxide layer for $300{ }^{\circ} \mathrm{C}$ treated sample compared to non-treated sample, nanotube formation is accelerated which results in fabrication of longer nanotubes.

In order to improve corrosion resistance of the titanium surface, the corrosion of surface is studied after anodization and thermal oxidation in presence of serum proteins, at physiological temperature. To determine corrosion rate multiple parameters such as corrosion potential, polarization resistance, open circuit potential, passivation current 
density and corrosion current density are verified. The results show that the highest corrosion resistance is provided by Anodized+Thermally Oxidized samples followed by Anodized samples, then Smooth samples and finally Thermally Oxidized samples. It is shown that heat treated nanotubular surface enhances corrosion resistance with reference to Ipass, Icorr, Ecorr, OCP and polarization and capacitance resistance behavior. It is suggested that this behavior is caused by presence of thicker oxide layer and higher surface area on crystalline nanotubular surface. 


\section{Chapter 9}

\section{Future Work}

In this chapter a few directions are suggested as the future work to extend the obtained results discussed in previous chapters. These directions include (i) biological evaluation, (ii) determining controlled drug release profiles, (iii) fabrication of smart drug eluting implants and (iv) optimization of surface properties.

\subsection{Biological evaluation}

As discussed in chapter 3 anodization conditions and following heat treatment of the surface influences its hydrophilicity over time. Considering the key role of surface hydrophilicity on cellular behavior, it is hypothesized that the cellular attachment and eventually osseointegration can be improved through controlling anodization voltage, anodization duration, heat treatment temperature and heat treatment duration. The future work includes investigation of effect of anodization and heat treatment conditions on attachment, survivability and proliferation of osteoblasts over a period of 3 months.

The results discussed in chapter 7 suggest that maximum corrosion resistance is provided by heat treated anodized samples followed by non heat treated anodized samples, non heat treated non anodized samples and finally non anodized heat treated samples. The future work of this study is to investigate the identical anodization and annealing conditions carried out in chapter 7 for osteoblast adhesion and viability in cathodic and anodic potentials. In order to achieve this aim, cellular adhesion and viability of osteoblasts on smooth surfaces, amorphous nanotubes, and rutile crystalline nanotube surfaces will be verified.

In addition to in-vitro tests, conducting in-vivo experiments is valuable in confirming the potential of nanotubular surfaces for biomedical applications. Performing 
animal studies will shed light on how optimization of nanotubular surface would improve osseointegration.

\subsection{Determining controlled drug release profiles}

As demonstrated in chapter 4, nanotubes can act as nanoreservoirs for local drug delivery to avascular tissue that is left after surgery. The results show nanotubes with high aspect ratio successfully prolong drug release for a short period of time. However for clinical purposes the drug release has to be further prolonged. In order to achieve this, biocompatible and biodegrable polymers can be employed to prevent burst release of drug as they gradually degrade, when exposed to aqueous physiologic environment. The future work includes loading drug into polymeric micelles. Then the drug-loaded micelles will be attached to the inner wall of nanotubes. Biological evaluation of the drug-loaded surface can clarify the effect of prolonged release of drug over time.

After surgery a sustained drug release over a specific time period is desirable for each particular drug. While presence of antibiotics is beneficial right after surgery, release of growth factors is desirable over long period of time. Therefore, it will be extremely valuable to determine the drug release profiles at various surface optimization conditions.

\subsection{Fabrication of smart drug eluting implants}

Although many factors influence implant success, implant failure is typically observed long after surgical operation. The failure is preceded by infection of surrounding tissue which causes a reduction in $\mathrm{pH}$. Therefore, it is possible to fabricate smart drug loaded implants that are able to react when $\mathrm{pH}$ is reduced and release their loaded drug. To achieve this objective, drug-loaded micelles can be attached to the inner wall of nanotubes as explained in section 9.3. Biodegradable polymers that are degraded faster in acidic conditions can be used for this purpose. In addition, Biodegradable

polymers that are degraded faster at body temperature can be used for fabrication of 
smart drug eluting implants that release their loaded drug after surgery and exposure to body temperature. For instance, polydopamine can be used for coating surface of biomaterial since it possesses functional groups that can conjugate either directly with drug or with drug loaded polymeric nanoparticles.

\subsection{Optimization of surface properties}

In chapter 5, the effect of hydrophilicity, roughness, structure and composition of various surfaces are correlated to the cellular behavior. It is shown that smooth and rough surfaces, before and after anodization, prior to and subsequent to heat treatment, provide different substrates for cellular interaction. Although anodized surfaces provide superior substrates for cellular interaction, detachment of nanotubes following exposure to micromotions of physiologic environment is likely. Consequently, the future work related to this study includes tribological investigation of various surfaces along with biological evaluations.

As shown in chapter 6, rutile crystalline structure of $\mathrm{TiO}_{2}$ (formed during heat treatment at $600{ }^{\circ} \mathrm{C}$ ) is potential to maximize surface hydrophilicity and eventually improve cellular response; whereas anatase crystalline structure of $\mathrm{TiO}_{2}$ (formed during heat treatment at $300{ }^{\circ} \mathrm{C}$ ) leads to formation of longer nanotubes. It is hypothesized that a thin layer of rutile can lead to formation of longer nanotubes. The future work includes performing heat treatment for as short as $15 \mathrm{~min}$ to generate rutile structure of $\mathrm{TiO}_{2}$ with a thickness comparable to that of formed during heat treatment at $300{ }^{\circ} \mathrm{C}$. The joint advantages of increased nanotube length and improved hydrophilicity can result in improved cellular interaction. Cellular tests will confirm the optimized condition for fabricating the surface. 
Appendix 


\section{Appendix 1: Copyright for reproduction of figure 2.2}

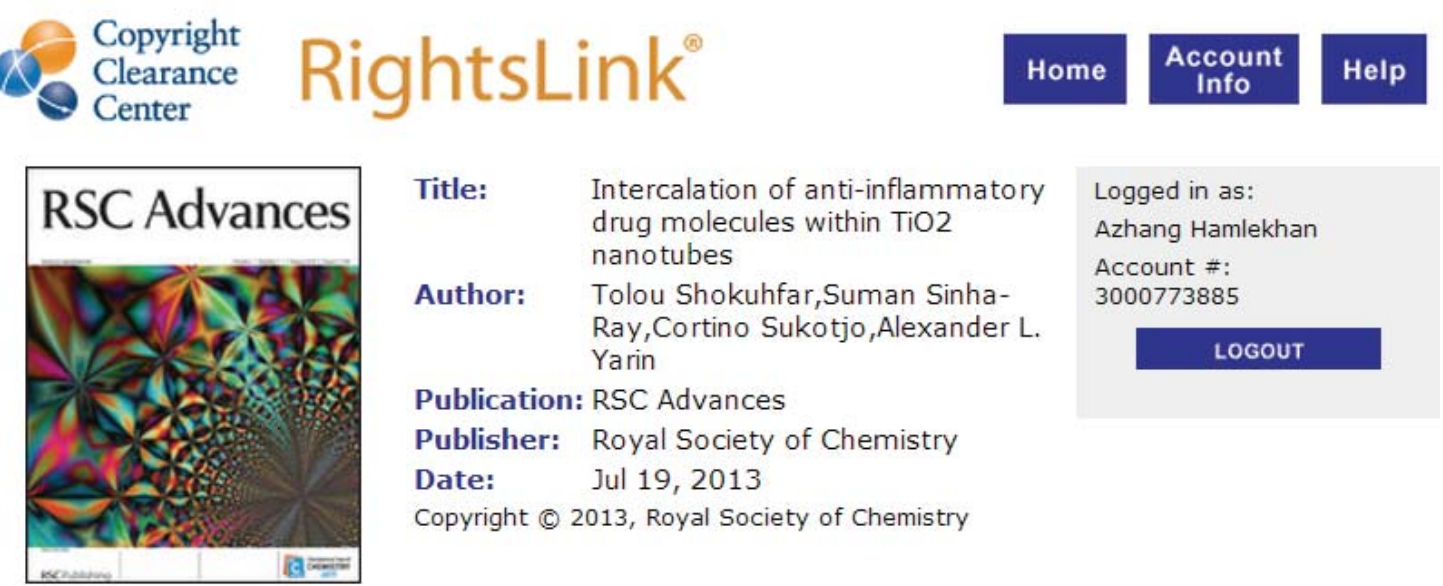

\section{Order Completed}

Thank you very much for your order.

This is a License Agreement between Azhang Hamlekhan ("You") and Royal Society of Chemistry. The license consists of your order details, the terms and conditions provided by Royal Society of Chemistry, and the payment terms and conditions.

Get the printable license.

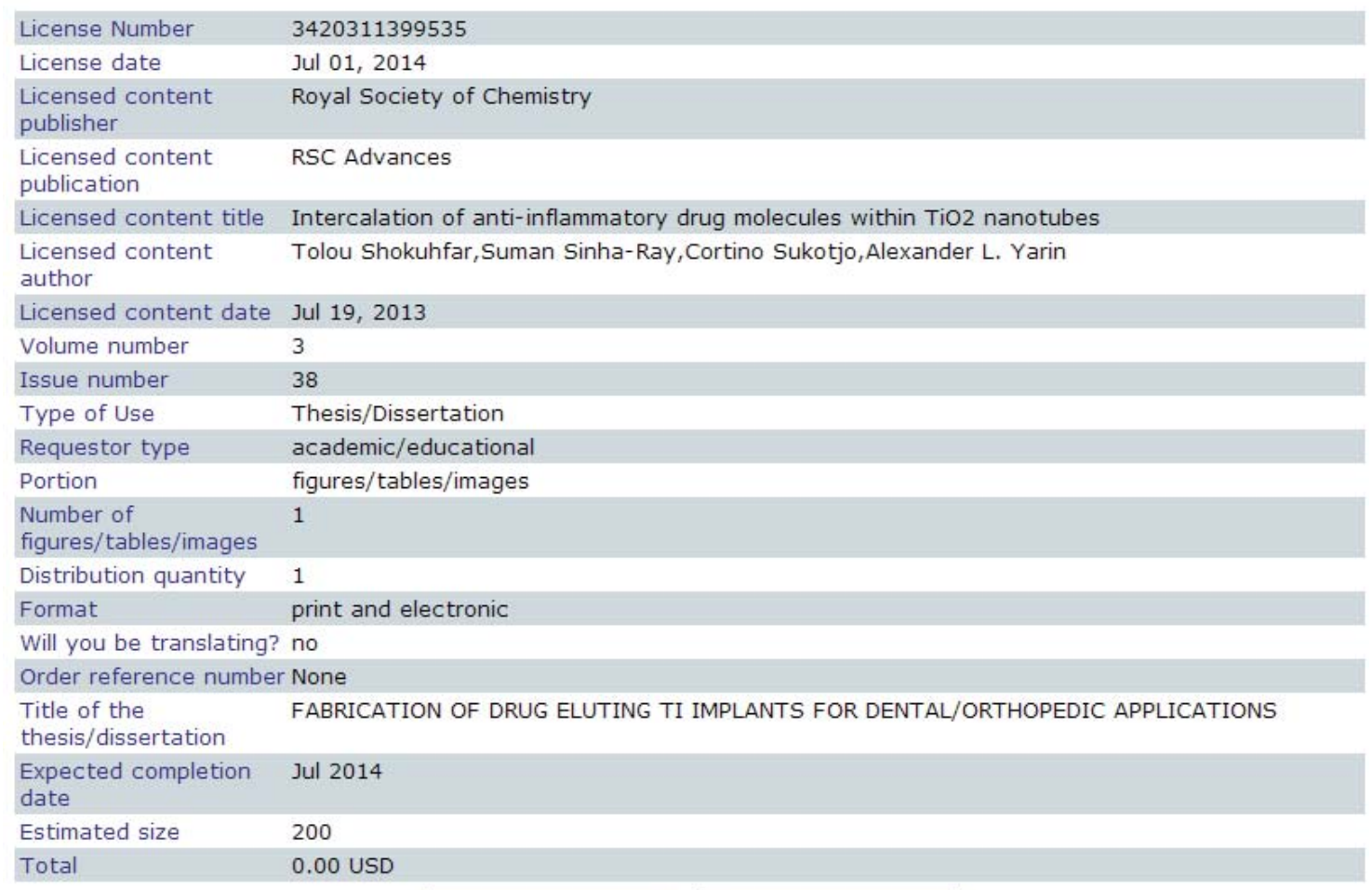

\section{ORDER MORE... CLOSE WINDOW}




\section{Appendix 2: Copyright for reproduction of figure 2.3}

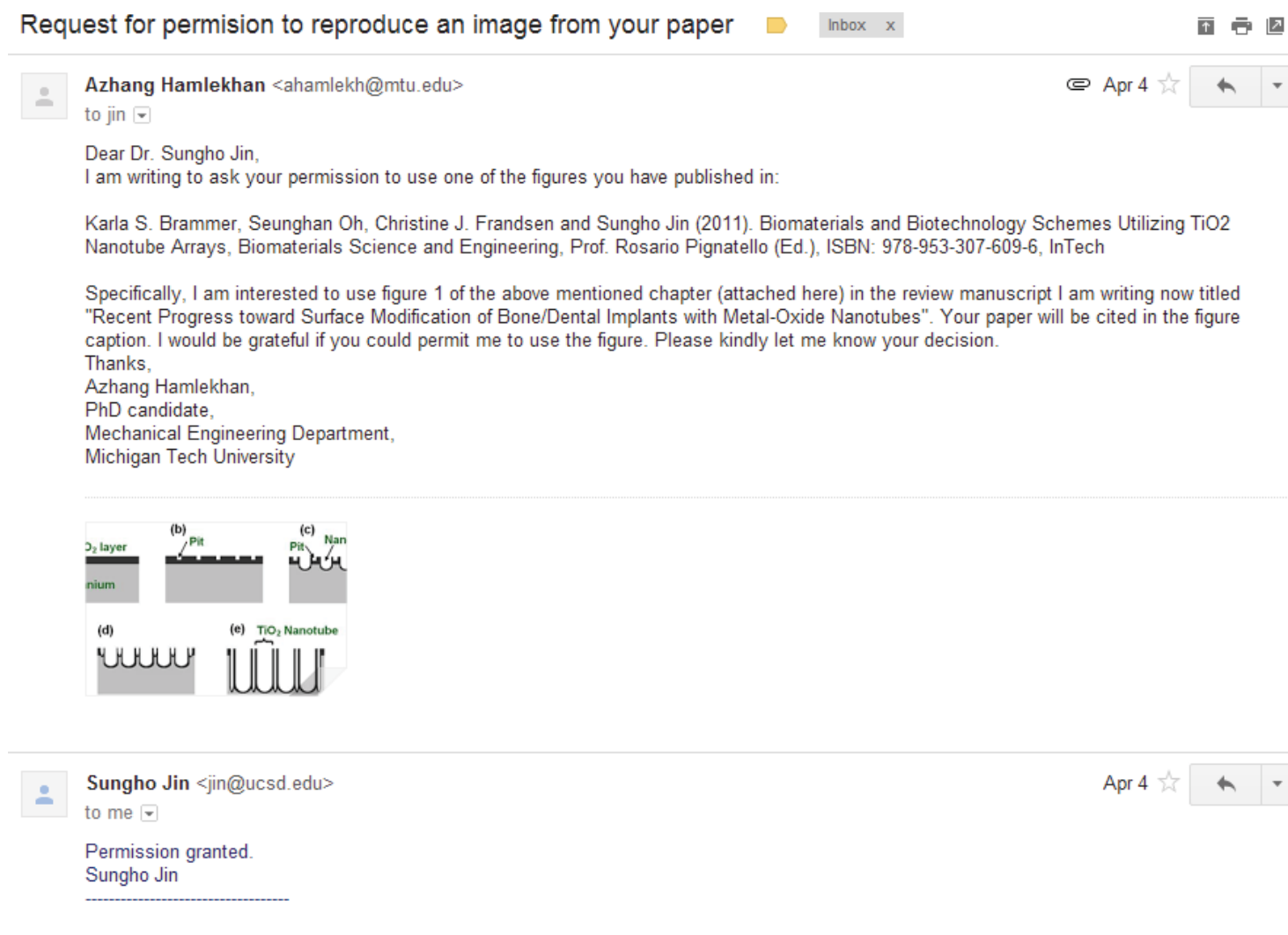

From: Azhang Hamlekhan [mailto:ahamlekh@mtu.edu]

Sent: Friday, April 04, 2014 12:31 PM

To: jin@UCSD.edu

Subject: Request for permision to reproduce an image from your paper

$\cdots$

- Azhang Hamlekhan <ahamlekh@mtu.edu>

to jin -

Apr 4

I really appreciate it.

$\cdots$

- Azhang Hamlekhan <ahamlekh@mtu.edu>

to jin $\nabla$

Dear Dr. Sungho Jin,

I am writing to ask your permission to re-use the same figure in my $\mathrm{PhD}$ dissertation. In fact, I would like to include the above mentioned

manuscript in my dissertation. I would be grateful if you could permit me to use the figure. Please kindly let me know your decision.

Thanks,

Azhang

$\cdots$

- Sungho Jin

7:54 PM (15 hours ago)

OK, permission granted.

- Azhang Hamlekhan <ahamlekh@mtu.edu>

to jin $\nabla$

I really appreciate it. 


\section{Appendix 3: Copyright for reproduction of figure 2.4}

\section{Copyright Clearance Center}

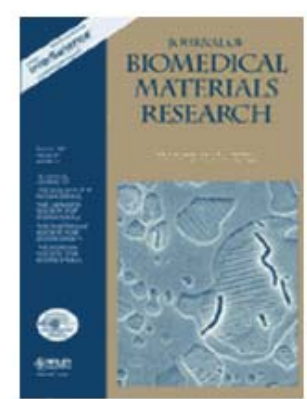

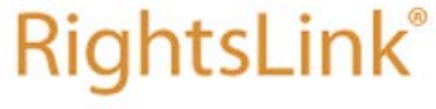

Title:

$\mathrm{TiO} 2$ nanotubes on Ti: Influence of nanoscale morphology on bone cell-materials interaction

Author:

Kakoli Das,Susmita Bose,Amit Bandyopadhyay

Publication: Journal of Biomedical Materials Research

Publisher: John Wiley and Sons

Date: May 21, 2008

Copyright (c) 2008 Wiley Periodicals, Inc.

\section{Order Completed}

Thank you very much for your order.

This is a License Agreement between Azhang Hamlekhan ("You") and John Wiley and Sons ("John Wiley and Sons"). The license consists of your order details, the terms and conditions provided by John Wiley and Sons, and the payment terms and conditions.

\section{Get the printable license.}

\begin{tabular}{|c|c|}
\hline License Number & 3419000996559 \\
\hline License date & Jun 30,2014 \\
\hline $\begin{array}{l}\text { Licensed content } \\
\text { publisher }\end{array}$ & John Wiley and Sons \\
\hline $\begin{array}{l}\text { Licensed content } \\
\text { publication }\end{array}$ & Journal of Biomedical Materials Research \\
\hline Licensed content title & TiO2 nanotubes on Ti: Influence of nanoscale morphology on bone cell-materials interaction \\
\hline Licensed copyright line & Copyright (C) 2008 Wiley Periodicals, Inc. \\
\hline $\begin{array}{l}\text { Licensed content } \\
\text { author }\end{array}$ & Kakoli Das,Susmita Bose,Amit Bandyopadhyay \\
\hline Licensed content date & May 21,2008 \\
\hline Start page & 225 \\
\hline End page & 237 \\
\hline Type of use & Dissertation/Thesis \\
\hline Requestor type & University/Academic \\
\hline Format & Print and electronic \\
\hline Portion & Figure/table \\
\hline $\begin{array}{l}\text { Number of } \\
\text { figures/tables }\end{array}$ & 1 \\
\hline $\begin{array}{l}\text { Original Wiley } \\
\text { figure/table number(s) }\end{array}$ & Figure 4 \\
\hline Will you be translating? & No \\
\hline $\begin{array}{l}\text { Title of your thesis / } \\
\text { dissertation }\end{array}$ & FABRICATION OF DRUG ELUTING TI IMPLANTS FOR DENTAL/ORTHOPEDIC APPLICATIONS \\
\hline $\begin{array}{l}\text { Expected completion } \\
\text { date }\end{array}$ & Jul 2014 \\
\hline $\begin{array}{l}\text { Expected size (number } \\
\text { of pages) }\end{array}$ & 200 \\
\hline Total & 0.00 USD \\
\hline
\end{tabular}




\section{Appendix 4: Copyright for reproduction of figure 2.5}

Copyright Clearance Center

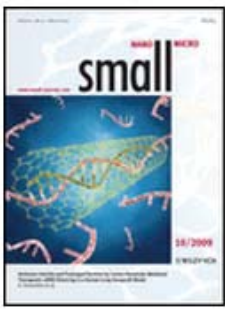

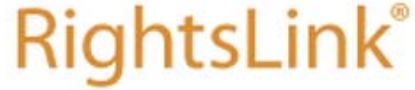

Title:

Title:

Author:

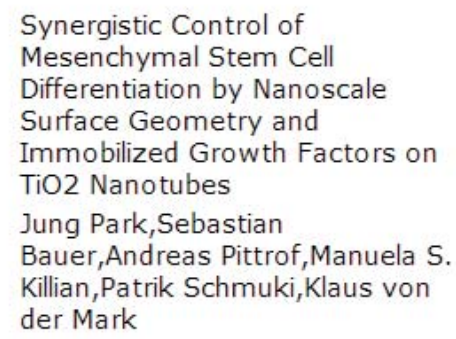

Synergistic Control of Mesenchymal Stem Cell

Differentiation by Nanoscale Surface Geometry and Immobilized Growth Factors on TiO2 Nanotubes

Bauer,Andreas Pittrof,Manuela S. Killian,Patrik Schmuki,Klaus von der Mark

Publication: Small

Publisher: John Wiley and Sons

Date: Nov 18, 2011

Copyright (c) 2012 WILEY-VCH Verlag GmbH \& Co. KGaA, Weinheim

\section{Order Completed}

Thank you very much for your order.

This is a License Agreement between Azhang Hamlekhan ("You") and John Wiley and Sons ("John Wiley and Sons"). The license consists of your order details, the terms and conditions provided by John Wiley and Sons, and the payment terms and conditions.

Get the printable license.

\begin{tabular}{|c|c|}
\hline License Number & 3419010786533 \\
\hline License date & Jun 30,2014 \\
\hline $\begin{array}{l}\text { Licensed content } \\
\text { publisher }\end{array}$ & John Wiley and Sons \\
\hline $\begin{array}{l}\text { Licensed content } \\
\text { publication }\end{array}$ & Small \\
\hline Licensed content title & $\begin{array}{l}\text { Synergistic Control of Mesenchymal Stem Cell Differentiation by Nanoscale Surface } \\
\text { Geometry and Immobilized Growth Factors on TiO2 Nanotubes }\end{array}$ \\
\hline Licensed copyright line & Copyright @ 2012 WILEY-VCH Verlag GmbH \& Co. KGaA, Weinheim \\
\hline $\begin{array}{l}\text { Licensed content } \\
\text { author }\end{array}$ & $\begin{array}{l}\text { Jung Park,Sebastian Bauer,Andreas Pittrof,Manuela S. Killian,Patrik Schmuki,Klaus von der } \\
\text { Mark }\end{array}$ \\
\hline Licensed content date & Nov 18,2011 \\
\hline Start page & 98 \\
\hline End page & 107 \\
\hline Type of use & Dissertation/Thesis \\
\hline Requestor type & University/Academic \\
\hline Format & Print and electronic \\
\hline Portion & Figure/table \\
\hline $\begin{array}{l}\text { Number of } \\
\text { figures/tables }\end{array}$ & 1 \\
\hline $\begin{array}{l}\text { Original Wiley } \\
\text { figure/table number(s) }\end{array}$ & Figure 4 \\
\hline \multicolumn{2}{|c|}{ Will you be translating? No } \\
\hline $\begin{array}{l}\text { Title of your thesis / } \\
\text { dissertation }\end{array}$ & FABRICATION OF DRUG ELUTING TI IMPLANTS FOR DENTAL/ORTHOPEDIC APPLICATIONS \\
\hline $\begin{array}{l}\text { Expected completion } \\
\text { date }\end{array}$ & Jul 2014 \\
\hline $\begin{array}{l}\text { Expected size (number } \\
\text { of pages) }\end{array}$ & 200 \\
\hline Total & 0.00 USD \\
\hline
\end{tabular}

ORDER MORE... CLOSE WINDOW 


\section{Appendix 5: Copyright for reproduction of figure 2.6}

Copyright Clearance Center

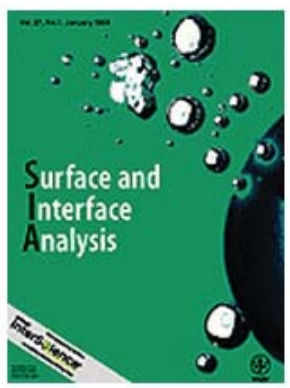

Title:

Author:

\section{Logged in as:} Azhang Hamlekhan Account \#: 3000773885 properties, hydroxyapatite growth and cell behaviors of $\mathrm{TiO} 2$ nanotubes

Yu Bai,Il Song Park,Hyeoung Ho

Park,Min Ho Lee,Tae Sung

Bae,Warwick Duncan,Michael

$$
\text { Swain }
$$

Publication: Surface \& Interface Analysis

Publisher: John Wiley and Sons

Date: Aug 5, 2010

Copyright (c) 2010 John Wiley \& Sons, Ltd.

\section{Order Completed}

Thank you very much for your order.

This is a License Agreement between Azhang Hamlekhan ("You") and John Wiley and Sons ("John Wiley and Sons"). The license consists of your order details, the terms and conditions provided by John Wiley and Sons, and the payment terms and conditions.

Get the printable license.

\begin{tabular}{|c|c|}
\hline License Number & 3419011234490 \\
\hline License date & Jun 30,2014 \\
\hline $\begin{array}{l}\text { Licensed content } \\
\text { publisher }\end{array}$ & John Wiley and Sons \\
\hline $\begin{array}{l}\text { Licensed content } \\
\text { publication }\end{array}$ & Surface \& Interface Analysis \\
\hline Licensed content title & $\begin{array}{l}\text { The effect of annealing temperatures on surface properties, hydroxyapatite growth and cell } \\
\text { behaviors of TiO2 nanotubes }\end{array}$ \\
\hline Licensed copyright line & Copyright (c) 2010 John Wiley \& Sons, Ltd. \\
\hline $\begin{array}{l}\text { Licensed content } \\
\text { author }\end{array}$ & $\begin{array}{l}\text { Yu Bai,Il Song Park, Hyeoung Ho Park, Min Ho Lee, Tae Sung Bae, Warwick Duncan, Michael } \\
\text { Swain }\end{array}$ \\
\hline Licensed content date & Aug 5, 2010 \\
\hline Start page & 998 \\
\hline End page & 1005 \\
\hline Type of use & Dissertation/Thesis \\
\hline Requestor type & University/Academic \\
\hline Format & Print and electronic \\
\hline Portion & Figure/table \\
\hline $\begin{array}{l}\text { Number of } \\
\text { figures/tables }\end{array}$ & 1 \\
\hline $\begin{array}{l}\text { Original Wiley } \\
\text { figure/table number(s) }\end{array}$ & Figure 11 \\
\hline \multicolumn{2}{|c|}{ Will you be translating? No } \\
\hline $\begin{array}{l}\text { Title of your thesis / } \\
\text { dissertation }\end{array}$ & FABRICATION OF DRUG ELUTING TI IMPLANTS FOR DENTAL/ORTHOPEDIC APPLICATIONS \\
\hline $\begin{array}{l}\text { Expected completion } \\
\text { date }\end{array}$ & Jul 2014 \\
\hline $\begin{array}{l}\text { Expected size (number } \\
\text { of pages) }\end{array}$ & 200 \\
\hline Total & 0.00 USD \\
\hline
\end{tabular}




\section{Appendix 6: Copyright for reproduction of figure 2.7}

Copyright

Clearance

Center

\section{RightsLink}

Title:

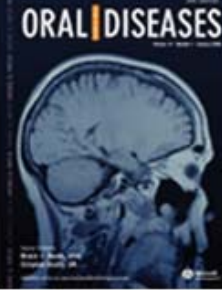

In vitro behavior of MC3T3-E1 preosteoblast with different annealing temperature titania nanotubes

Author: WQ Yu, YL Zhang, XQ Jiang, FQ Zhang

Publication: Oral Diseases

Publisher: John Wiley and Sons

Date: Jun 29, 2010

(C) 2010 John Wiley \& Sons A/S

\section{Order Completed}

Thank you very much for your order.

This is a License Agreement between Azhang Hamlekhan ("You") and John Wiley and Sons ("John Wiley and Sons"). The license consists of your order details, the terms and conditions provided by John Wiley and Sons, and the payment terms and conditions.

Get the printable license.

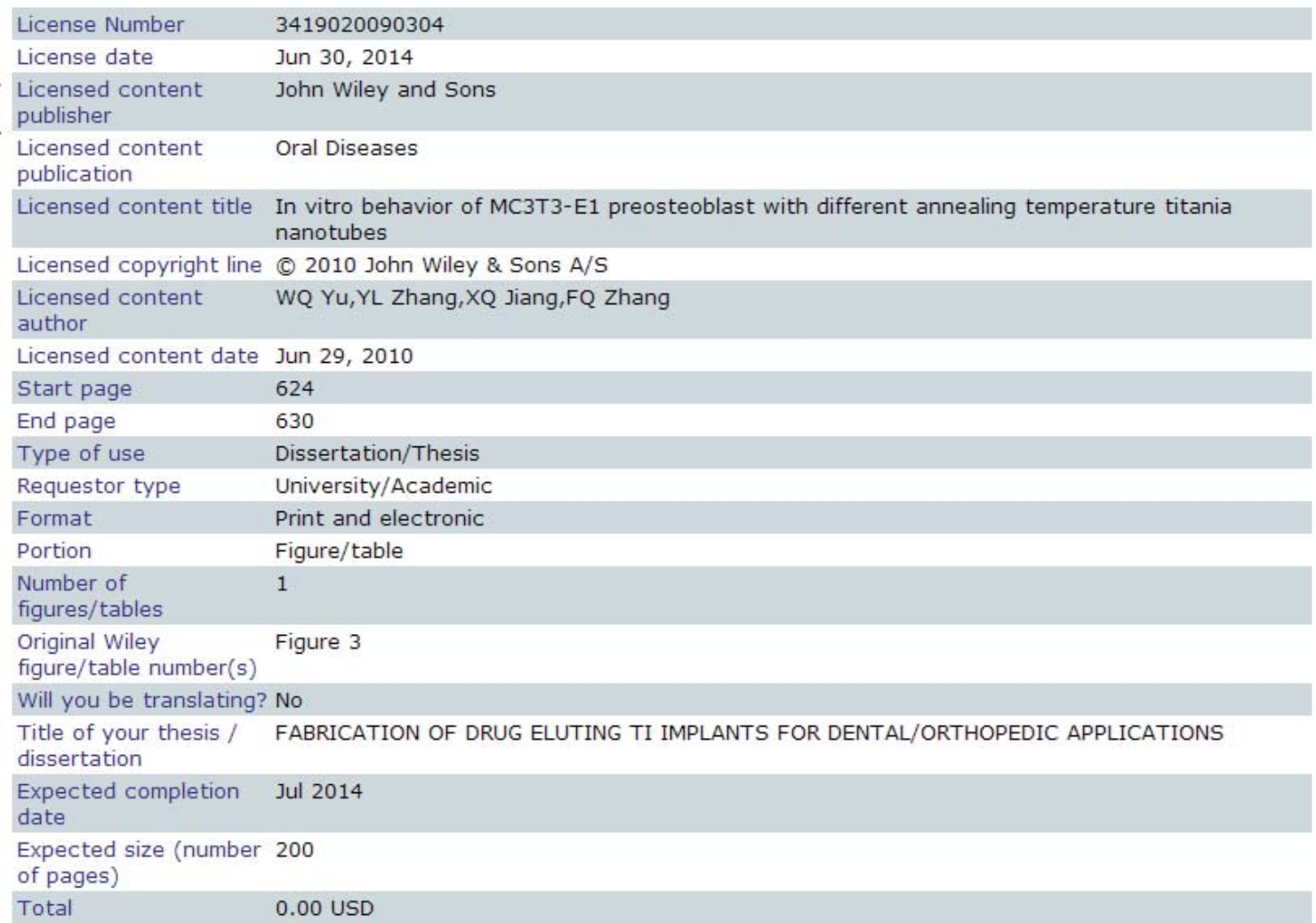




\title{
Appendix 7: Copyright for reproduction of figure 2.8
}

\author{
2. Azhang Hamlekhan <ahamlekh@mtu.edu> \\ 12:59 PM (4 hours ago) \\ $h$ \\ to Tolou $r$ \\ Dear professor Shokhfar, \\ I am writing to ask your permission to use one of the figures you have published in your PhD dissertation: \\ STRUCTURAL AND SURFACE PROPERTY CHARACTERIZATION OF TITANIUM DIOXIDE NANOTUBES FOR ORTHOPEDIC IMPLANTS \\ Specifically, I am interested to use figure 3.2 of the above mentioned publication in my PhD dissertation titled "FABRICATION OF DRUG \\ ELUTING TI IMPLANTS FOR DENTAL/ORTHOPEDIC APPLICATIONS". Your dissertation will be cited in the figure caption. I would be \\ grateful if you could permit me to use the figure. Please kindly let me know your decision. \\ Thanks, \\ Azhang Hamlekhan \\ Tolou Shokuhfar PhD \\ to me $\nabla$ \\ 4:38 PM (25 minutes ago) \\ Permission granted \\ Thanks \\ TS \\ Tolou Shokuhfar, Ph.D. \\ Assistant Professor \\ Director, In-Situ Nanomedicine Laboratory \\ Department of Mechanical Engineering, Department of Biomedical Engineering, Michigan Technological University \\ Adjunct Assistant Professor, College Of Dentistry, University of Illinois at Chicago, \\ Adjunct Assistant Professor, Department of Physics, University of Illinois at Chicago \\ Phone: (906) 3707657 \\ Fax: 1906$) 4872822$ \\ http://www. me.mtuedu/meem facultvbio/Tolou, Shokuhfar. html
}




\section{Appendix 8: Copyright for reproduction of figure 2.9}

Copyright Clearance Center

\section{RightsLink}

Title:

Intercalation of anti-inflammatory drug molecules within $\mathrm{TiO} 2$ nanotubes

Author:

Tolou Shokuhfar,Suman SinhaRay,Cortino Sukotjo,Alexander L. Yarin

Publication: RSC Advances

Publisher: Royal Society of Chemistry

Date: Jul 19, 2013

Copyright (c) 2013, Royal Society of Chemistry
Logged in as: Azhang Hamlekhan Account \#: 3000773885

LOGOUT

\section{Order Completed}

Thank you very much for your order.

This is a License Agreement between Azhang Hamlekhan ("You") and Royal Society of Chemistry. The license consists of your order details, the terms and conditions provided by Royal Society of Chemistry, and the payment terms and conditions.

Get the printable license.

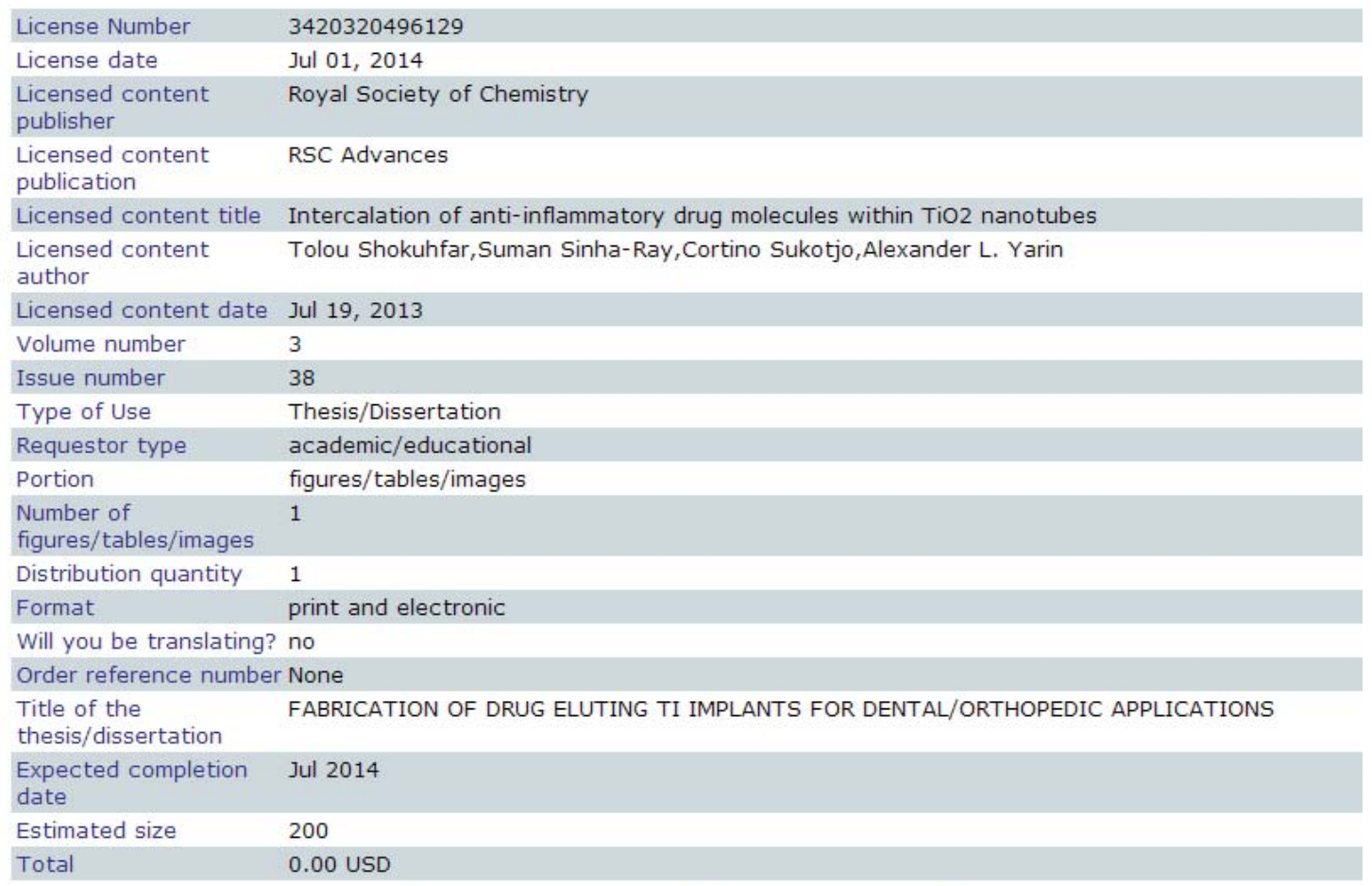




\section{Appendix 9: Copyright for the Manuscript Submitted to the journal of PLOS ONE (chapter 3)}

\begin{tabular}{r|r|r|} 
Subject Areas & For Authors & About Us \\
& Senrch & and
\end{tabular}

\section{Open-Access License}

\section{No Permission Required}

PLOS applies the Creative Commons Attribution (CC BY) license to all works we publish (read the human-readable summary or the full license legal code). Under the CC BY license, authors retain ownership of the copyright for their article, but authors allow anyone to download, reuse, reprint, modify, distribute, and/or copy

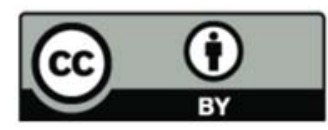
articles in PLOS journals, so long as the original authors and source are cited. No permission is required from the authors or the publishers.

In most cases, appropriate attribution can be provided by simply citing the original article (e.g., Kaltenbach LS et al. (2007) Huntingtin Interacting Proteins Are Genetic Modifiers of Neurodegeneration. PLOS Genet 3(5): e82. doi:10.1371/journal.pgen.0030082). If the item you plan to reuse is not part of a published article (e.g., a featured issue image), then please indicate the originator of the work, and the volume, issue, and date of the journal in which the item appeared. For any reuse or redistribution of a work, you must also make clear the license terms under which the work was published. 


\section{Appendix 10: Copyright for the Manuscript Submitted to the journal of Advanced Healthcare Materials (chapter 4)}

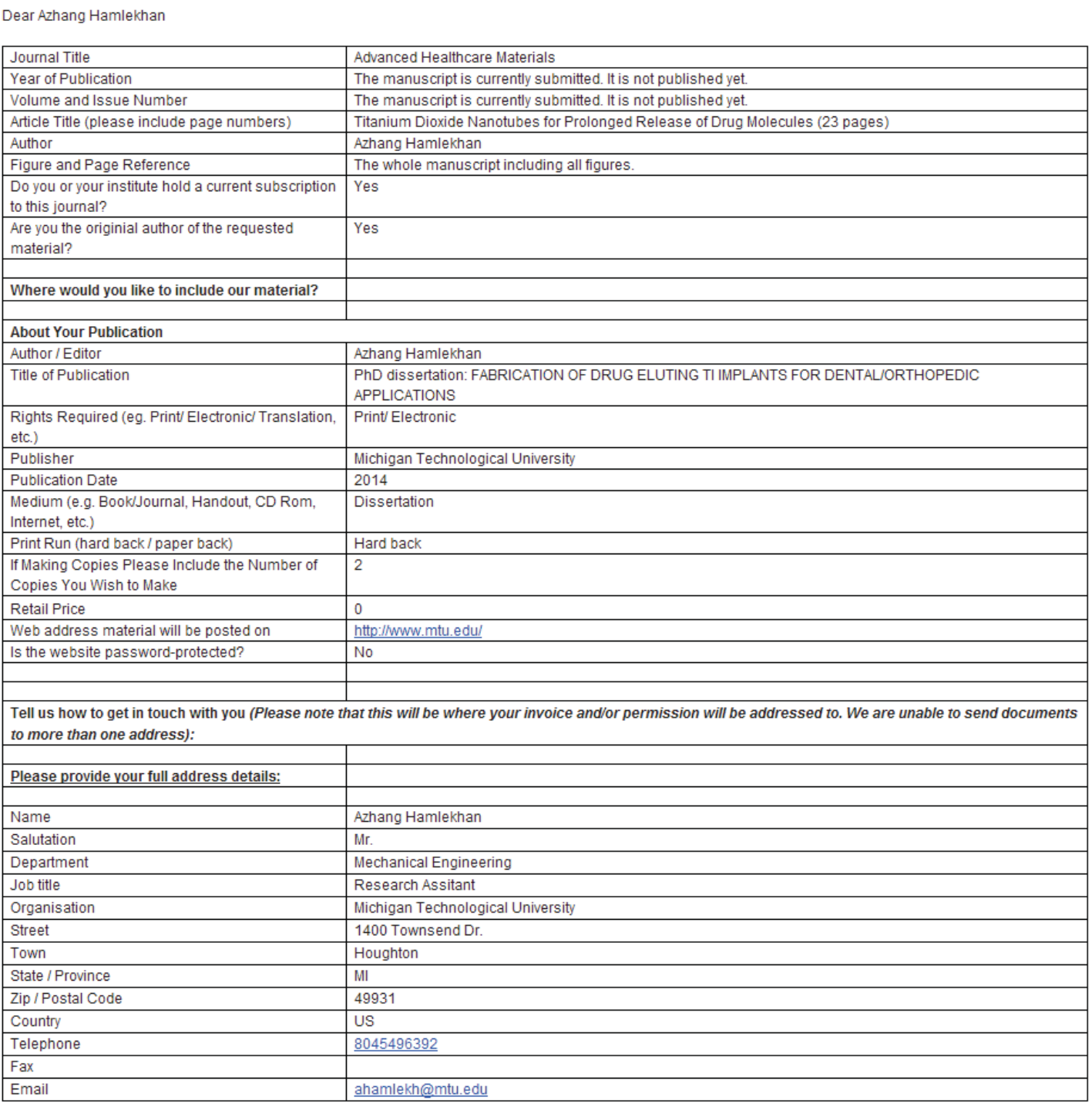




\title{
Appendix 11: Copyright for the Manuscript Submitted to the journal of Journal of Materials Chemistry B (chapter 5)
}

RSC| $\begin{aligned} & \text { Advancing the } \\ & \text { Chemical Sciences }\end{aligned}$

\author{
Royal Society of Chemistry \\ Thomas Graham House \\ Science Park \\ Milton Road \\ Cambridge
CB4 OWF \\ Tel: +44 (0)1223420 066 \\ Fax: $+44(0) 1223423623$ \\ Email: contracts-copyright@rsc.org \\ www.rsc.org
}

Acknowledgements to be used by RSC authors

Authors of RSC books and journal articles can reproduce material (for example a figure) from the RSC publication in a non-RSC publication, including theses, without formally requesting permission providing that the correct acknowledgement is given to the RSC publication. This permission extends to reproduction of large portions of text or the whole article or book chapter when being reproduced in a thesis.

The acknowledgement to be used depends on the RSC publication in which the material was published and the form of the acknowledgements is as follows:

- For material being reproduced from an article in New Journal of Chemistry the acknowledgement should be in the form:

- [Original citation] - Reproduced by permission of The Royal Society of Chemistry (RSC) on behalf of the Centre National de la Recherche Scientifique (CNRS) and the RSC

- For material being reproduced from an article Photochemical \& Photobiological Sciences the acknowledgement should be in the form:

- [Original citation] - Reproduced by permission of The Royal Society of Chemistry (RSC) on behalf of the European Society for Photobiology, the European Photochemistry Association, and RSC

- For material being reproduced from an article in Physical Chemistry Chemical Physics the acknowledgement should be in the form:

- [Original citation] - Reproduced by permission of the PCCP Owner Societies

- For material reproduced from books and any other journal the acknowledgement should be in the form:

- [Original citation] - Reproduced by permission of The Royal Society of Chemistry

The acknowledgement should also include a hyperlink to the article on the RSC website.

The form of the acknowledgement is also specified in the RSC agreement/licence signed by the corresponding author.

Except in cases of republication in a thesis, this express permission does not cover the reproduction of large portions of text from the RSC publication or reproduction of the whole article or book chapter.

A publisher of a non-RSC publication can use this document as proof that permission is granted to use the material in the non-RSC publication. 


\section{Appendix 12: Copyright for the Manuscript Submitted to the Journal of Oral Implantology (chapter 6)}

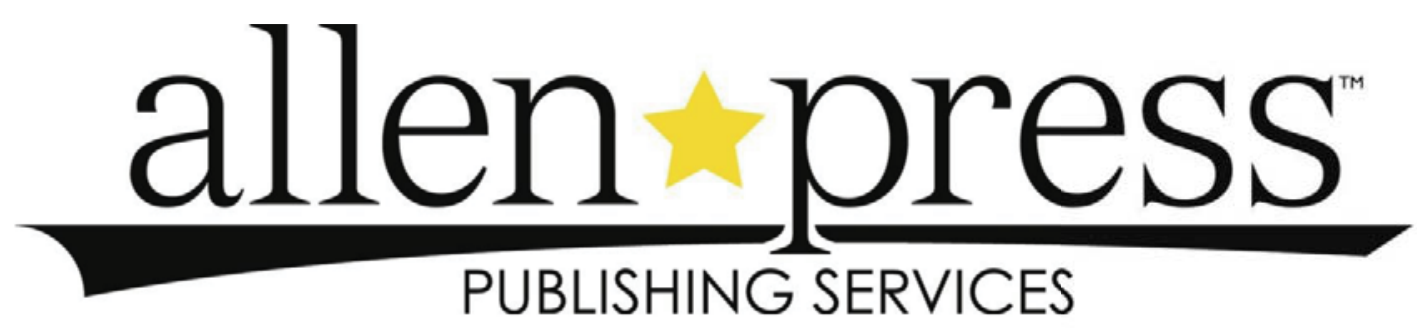

Date: June 12, 2014

Dear Azhang Hamlekhan,

On behalf of Allen Press Publishing Services, I am pleased to grant permission to you for the reprinting of the following:

"A Novel Investigation of the Formation of $\mathrm{TiO}_{2}$ Nanotubes on Thermally Formed Oxide of Ti-6Al-4V" by Butt, et al. appearing in Journal of Oral Implantology In-Press (2014).

For use in PhD dissertation by Hamlekhan.

This permission is a one-time, non-exclusive, electronic worldwide grant for English language use as described in this letter, and is subject to the following conditions:

I. No payment required.

2. Each copy containing our material that you reproduce or distribute must bear the appropriate copyright information, crediting the author, journal, and publisher (Journal of Oral Implantology Allen Press Publishing Services).

3. The original author's permission must also be independently obtained before permission is granted.

If these terms are acceptable, please sign and date, and fax back to my attention at 785-843-1853. This permission will be effective upon our receipt of the signed contract. If applicable, when sending payment, please make clear reference to our title and author. Materials should be addressed to the Journal of Oral Implantology, c/o Nicole Richter, P.O. Box 1897, Lawrence, KS 66044.

Sincerely,

Nicole Richter

Publishing Specialist

Allen Press Publishing Services

AGREED: 


\section{Appendix 13: Copyright for the Manuscript Submitted to the Journal of Electrochimica Acta (chapter 7)}

$\therefore$ Samantray, Banita (ELS-CHN) <b.samantray@reed $\epsilon$

May 22

to me $r$

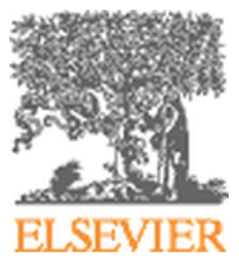

Dear Azhang Hamlekhan

Please note that as one of the Authors of this article, you retain the right to include the journal article, in full or in part, in a thesis or dissertation.

You do not require permission to do so.

For full details of your rights as a Journal Author, please visit:

http://www.elsevier.com/wps/find/authorsview.authors/ copyright\#whatrights

Your retained rights allow you to submit your article in electronic format and to post this Elsevier article online if it is embedded within your thesis. You are also permitted to post your Author Accepted Manuscript online however posting of the final published article is prohibited.

Please refer to Elsevier's Posting Policy for further information:

http://www.elsevier.com/wps/find/authors.authors/postingpolicy

Please feel free to contact me if you have any queries.

Kind regards

Regards

Banita Samantray

Global Rights Department

\section{Elsevier}

(A division of Reed Elsevier India Pvt. Ltd.)

Ascendas International Tech Park | Crest Building $-12^{\text {th }}$ Floor | Taramani Road |

Taramani | Chennai 600113 | India

Tel: +914442994667 | Fax: +914442994701

E-mail: b.samantray@reedelsevier.com I url: www.elsevier.com 


\section{Reference List}

1. Navarro M, Michiardi A, Castano O, Planell JA (2008) Biomaterials in orthopaedics. Journal of the Royal Society Interface 5: 1137-1158.

2. Hamlekhan A, Mozafari M, Nezafati N, Azami M, Hadipour H (2010) A Proposed Fabrication Method of Novel PCL-GEL-HAp Nanocomposite Scaffolds for Bone Tissue Engineering Applications. Advanced Composites Letters 19: 123-130.

3. Hamlekhan A, Moztarzadeh F, Mozafari M, Azami M, Nezafati N (2011) Preparation of laminated poly(epsilon-caprolactone)-gelatin-hydroxyapatite nanocomposite scaffold bioengineered via compound techniques for bone substitution. Biomatter 1: 91-101.

4. Azhang Hamlehkhan MM, Nader Nezafati, Mahmoud Azami, Ali Samadikuchaksaraei (2011) Novel Bioactive Poly( $\varepsilon$-caprolactone)-Gelatin-Hydroxyapatite Nanocomposite Scaffolds for Bone Regeneration. Key Engineering Materials 493 - 494: 909-915.

5. Niinomi M (2008) Metallic biomaterials. J Artif Organs 11: 105-110.

6. Minagar S, Berndt CC, Wang J, Ivanova E, Wen C (2012) A review of the application of anodization for the fabrication of nanotubes on metal implant surfaces. Acta Biomaterialia 8: 2875-2888.

7. Niespodziana K, Jurczyk K, Jurczyk M (2008) The synthesis of titanium alloys for biomedical applications. Reviews on Advanced Materials Science 18: 236-240.

8. Barao VAR, Mathew MT, Assuncao WG, Yuan JC-C, Wimmer MA, et al. (2012) Stability of cp-Ti and Ti-6Al-4V alloy for dental implants as a function of saliva pH - an electrochemical study. Clinical Oral Implants Research 23: 1055-1062.

9. Yu WQ, Zhang YL, Jiang XQ, Zhang FQ (2010) In vitro behavior of MC3T3-E1 preosteoblast with different annealing temperature titania nanotubes. Oral Diseases 16: 624-630.

10. Das K, Bose S, Bandyopadhyay A (2009) TiO2 nanotubes on Ti: Influence of nanoscale morphology on bone cell-materials interaction. Journal of Biomedical Materials Research Part A 90A: 225-237. 
11. Chen ZX, Takao Y, Wang WX, Matsubara T, Ren LM (2009) Surface characteristics and in vitro biocompatibility of titanium anodized in a phosphoric acid solution at different voltages. Biomedical Materials 4.

12. Yu W-q, Qiu J, Xu L, Zhang F-q (2009) Corrosion behaviors of TiO2 nanotube layers on titanium in Hank's solution. Biomedical Materials 4.

13. Chen GJ, Wang Z, Bai H, Li JM, Cai H (2009) A preliminary study on investigating the attachment of soft tissue onto micro-arc oxidized titanium alloy implants. Biomedical Materials 4.

14. Kim H, Choi S-H, Ryu J-J, Koh S-Y, Park J-H, et al. (2008) The biocompatibility of SLA-treated titanium implants. Biomedical Materials 3.

15. Lausmaa J (1996) Surface spectroscopic characterization of titanium implant materials. Journal of Electron Spectroscopy and Related Phenomena 81: 343-361.

16. Liu XY, Chu PK, Ding CX (2004) Surface modification of titanium, titanium alloys, and related materials for biomedical applications. Materials Science \& Engineering R-Reports 47: 49-121.

17. Hu X, Shen H, Shuai K, Zhang E, Bai Y, et al. (2011) Surface bioactivity modification of titanium by $\mathrm{CO} 2$ plasma treatment and induction of hydroxyapatite: In vitro and in vivo studies. Applied Surface Science 257: 18131823.

18. Barao VA, Mathew MT, Assuncao WG, Yuan JC, Wimmer MA, et al. (2011) The Role of Lipopolysaccharide on the Electrochemical Behavior of Titanium. Journal of Dental Research 90: 613-618.

19. Webster TJ, Ejiofor JU (2004) Increased osteoblast adhesion on nanophase metals: Ti, Ti6Al4V, and CoCrMo. Biomaterials 25: 4731-4739.

20. Rajyalakshmi A, Ercan B, Balasubramanian K, Webster TJ (2011) Reduced adhesion of macrophages on anodized titanium with select nanotube surface features. International Journal of Nanomedicine 6: 1765-1771.

21. Yao C, Perla V, McKenzie JL, Slamovich EB, Webster TJ (2005) Anodized Ti and $\mathrm{Ti}(6) \mathrm{Al}(4) \mathrm{V}$ Possessing Nanometer Surface Features Enhances Osteoblast Adhesion. Journal of Biomedical Nanotechnology 1: 68-73. 
22. Shokuhfar T, Arumugam GK, Heiden PA, Yassar RS, Friedrich C (2009) Direct Compressive Measurements of Individual Titanium Dioxide Nanotubes. Acs Nano 3: 3098-3102.

23. Asthana A, Shokuhfar T, Gao Q, Heiden P, Friedrich C, et al. (2010) A study on the modulation of the electrical transport by mechanical straining of individual titanium dioxide nanotube. Applied Physics Letters 97.

24. Shokuhfar T, Gao Q, Ashtana A, Walzack K, Heiden P, et al. (2010) Structural instabilities in TiO2 nanotubes. Journal of Applied Physics 108.

25. Asthana A, Shokuhfar T, Gao Q, Heiden PA, Yassar RS (2012) Deformation-driven electrical transport in amorphous TiO2 nanotubes. Applied Physics a-Materials Science \& Processing 109: 127-132.

26. Debmalya Ganguly RS-Y, Tolou Shokuhfar (2014) Recent Advances in Nanotubes for Orthopedic Implants. J Nanotech Smart Mater Vol 1: 201.

27. Tsuchiya H, Macak JM, Muller L, Kunze J, Muller F, et al. (2006) Hydroxyapatite growth on anodic TiO2 nanotubes. Journal of Biomedical Materials Research Part A 77A: 534-541.

28. Oh SH, Finones RR, Daraio C, Chen LH, Jin SH (2005) Growth of nano-scale hydroxyapatite using chemically treated titanium oxide nanotubes. Biomaterials 26: 4938-4943.

29. von Wilmowsky C, Bauer S, Roedl S, Neukam FW, Schmuki P, et al. (2012) The diameter of anodic $\mathrm{TiO} 2$ nanotubes affects bone formation and correlates with the bone morphogenetic protein-2 expression in vivo. Clinical Oral Implants Research 23: 359-366.

30. Kim HW, Koh YH, Li LH, Lee S, Kim HE (2004) Hydroxyapatite coating on titanium substrate with titania buffer layer processed by sol-gel method. Biomaterials 25: 2533-2538.

31. Bjursten LM, Rasmusson L, Oh S, Smith GC, Brammer KS, et al. (2010) Titanium dioxide nanotubes enhance bone bonding in vivo. Journal of Biomedical Materials Research Part A 92A: 1218-1224. 
32. Yamano S, Al-Sowygh ZH, Gallucci GO, Wada K, Weber H-P, et al. (2011) Early peri-implant tissue reactions on different titanium surface topographies. Clinical Oral Implants Research 22: 815-819.

33. Simchi A, Tamjid E, Pishbin F, Boccaccini AR (2011) Recent progress in inorganic and composite coatings with bactericidal capability for orthopaedic applications. Nanomedicine-Nanotechnology Biology and Medicine 7: 22-39.

34. Brammer KS, Oh S, Cobb CJ, Bjursten LM, van der Heyde H, et al. (2009) Improved bone-forming functionality on diameter-controlled $\mathrm{TiO} 2$ nanotube surface. Acta Biomaterialia 5: 3215-3223.

35. Bae C, Yoo H, Kim S, Lee K, Kim J, et al. (2008) Template-directed synthesis of oxide nanotubes: Fabrication, characterization, and applications. Chemistry of Materials 20: 756-767.

36. Swami N, Cui Z, Nair LS (2011) Titania Nanotubes: Novel Nanostructures for Improved Osseointegration. Journal of Heat Transfer-Transactions of the Asme 133.

37. Ou H-H, Lo S-L (2007) Review of titania nanotubes synthesized via the hydrothermal treatment: Fabrication, modification, and application. Separation and Purification Technology 58: 179-191.

38. Yan C, Liu J, Liu F, Wu J, Gao K, et al. (2008) Tube Formation in Nanoscale Materials. Nanoscale Research Letters 3: 473-480.

39. Yan CL, Liu J, Liu F, Wu JS, Gao K, et al. (2008) Tube Formation in Nanoscale Materials. Nanoscale Research Letters 3: 473-480.

40. Macak JM, Tsuchiya H, Taveira L, Ghicov A, Schmuki P (2005) Self-organized nanotubular oxide layers on Ti-6A1-7Nb and Ti-6A1-4V formed by anodization in NH4F solutions. Journal of Biomedical Materials Research Part A 75A: 928933.

41. Shokuhfar T, Sinha-Ray S, Sukotjo C, Yarin AL (2013) Intercalation of antiinflammatory drug molecules within $\mathrm{TiO} 2$ nanotubes. Rsc Advances 3: 1738017386. 
42. Macak JM, Tsuchiya H, Ghicov A, Yasuda K, Hahn R, et al. (2007) TiO2 nanotubes: Self-organized electrochemical formation, properties and applications. Current Opinion in Solid State \& Materials Science 11: 3-18.

43. Roy P, Berger S, Schmuki P (2011) TiO2 Nanotubes: Synthesis and Applications. Angewandte Chemie-International Edition 50: 2904-2939.

44. Ghicov A, Schmuki P (2009) Self-ordering electrochemistry: a review on growth and functionality of $\mathrm{TiO} 2$ nanotubes and other self-aligned MOx structures. Chemical Communications: 2791-2808.

45. Albu SP, Ghicov A, Aldabergenova S, Drechsel P, LeClere D, et al. (2008) Formation of Double-Walled TiO2 Nanotubes and Robust Anatase Membranes. Advanced Materials 20: 4135-+.

46. By Karla S. Brammer SO, Christine J. Frandsen and Sungho Jin (2011) Biomaterials and Biotechnology Schemes Utilizing TiO2 Nanotube Arrays. Chapter 9.

47. Massia SP (1999) Cell-extracellular matrix interactions relevant to vascular tissue engineering. Tissue Engineering Prosthetic Vascular Grafts.

48. Oh S, Jin S (2006) Titanium oxide nanotubes with controlled morphology for enhanced bone growth. Materials Science \& Engineering C-Biomimetic and Supramolecular Systems 26: 1301-1306.

49. Park J, Bauer S, von der Mark K, Schmuki P (2007) Nanosize and vitality: TiO2 nanotube diameter directs cell fate. Nano Letters 7: 1686-1691.

50. Nourmohammadzadeh M, Lo JF, Bochenek M, Mendoza-Elias JE, Wang Q, et al. (2013) Microfluidic Array with Integrated Oxygenation Control for Real-Time Live-Cell Imaging: Effect of Hypoxia on Physiology of Microencapsulated Pancreatic Islets. Analytical Chemistry 85: 11240-11249.

51. Eshkeiti A, Narakathu BB, Reddy ASG, Moorthi A, Atashbar MZ, et al. (2012) Detection of heavy metal compounds using a novel inkjet printed surface enhanced Raman spectroscopy (SERS) substrate. Sensors and Actuators BChemical 171: 705-711.

52. Tami AE, Schaffler MB, Tate MLK (2003) Probing the tissue to subcellular level structure underlying bone's molecular sieving function. Biorheology 40: 577-590. 
53. Tan AW, Pingguan-Murphy B, Ahmad R, Akbar SA (2012) Review of titania nanotubes: Fabrication and cellular response. Ceramics International 38: 44214435 .

54. Oh S, Daraio C, Chen LH, Pisanic TR, Finones RR, et al. (2006) Significantly accelerated osteoblast cell growth on aligned $\mathrm{TiO} 2$ nanotubes. Journal of Biomedical Materials Research Part A 78A: 97-103.

55. Brammer KS, Frandsen CJ, Jin S (2012) TiO2 nanotubes for bone regeneration. Trends in Biotechnology 30: 315-322.

56. Oh S, Brammer KS, Li YSJ, Teng D, Engler AJ, et al. (2009) Stem cell fate dictated solely by altered nanotube dimension. Proceedings of the National Academy of Sciences of the United States of America 106: 2130-2135.

57. Park J, Bauer S, Schlegel KA, Neukam FW, von der Mark K, et al. (2009) TiO2 Nanotube Surfaces: $15 \mathrm{~nm}$ - An Optimal Length Scale of Surface Topography for Cell Adhesion and Differentiation. Small 5: 666-671.

58. Bauer S, Park J, Faltenbacher J, Berger S, von der Mark K, et al. (2009) Size selective behavior of mesenchymal stem cells on $\mathrm{ZrO} 2$ and $\mathrm{TiO} 2$ nanotube arrays. Integrative Biology 1: 525-532.

59. Park J, Bauer S, Pittrof A, Killian MS, Schmuki P, et al. (2012) Synergistic Control of Mesenchymal Stem Cell Differentiation by Nanoscale Surface Geometry and Immobilized Growth Factors on TiO2 Nanotubes. Small 8: 98-107.

60. Brammer KS, Oh S, Frandsen CJ, Varghese S, Jin S (2010) Nanotube surface triggers increased chondrocyte extracellular matrix production. Materials Science \& Engineering C-Materials for Biological Applications 30: 518-525.

61. Niinomi M (2008) Mechanical biocompatibilities of titanium alloys for biomedical applications. Journal of the Mechanical Behavior of Biomedical Materials 1: 3042.

62. Wang K (1996) The use of titanium for medical applications in the USA. Materials Science and Engineering a-Structural Materials Properties Microstructure and Processing 213: 134-137. 
63. Peng L, Eltgroth ML, LaTempa TJ, Grimes CA, Desai TA (2009) The effect of TiO2 nanotubes on endothelial function and smooth muscle proliferation. Biomaterials 30: 1268-1272.

64. Park J, Bauer S, Schmuki P, von der Mark K (2009) Narrow Window in Nanoscale Dependent Activation of Endothelial Cell Growth and Differentiation on TiO2 Nanotube Surfaces. Nano Letters 9: 3157-3164.

65. Giordano C, Saino E, Rimondini L, Pedeferri MP, Visai L, et al. (2011) Electrochemically induced anatase inhibits bacterial colonization on Titanium Grade 2 and Ti6A14V alloy for dental and orthopedic devices. Colloids and Surfaces B-Biointerfaces 88: 648-655.

66. Ercan B, Taylor E, Alpaslan E, Webster TJ (2011) Diameter of titanium nanotubes influences anti-bacterial efficacy. Nanotechnology 22: 11.

67. Giordano C, Sandrini E, Del Curto B, Signorelli E, Rondelli G, et al. (2004) Titanium for osteointegration: Comparison between a novel biomimetic treatment and commercially exploited surfaces. Journal of applied biomaterials \& biomechanics : JABB 2: 35-44.

68. Christensen GD, Simpson WA, Younger JJ, Baddour LM, Barrett FF, et al. (1985) Adherence of coagulase-negative staphylococci to plastic tissue culture plates: a quantitative model for the adherence of staphylococci to medical devices. Journal of Clinical Microbiology 22: 996-1006.

69. Chen CS (2008) Mechanotransduction - a field pulling together? Journal of Cell Science 121: 3285-3292.

70. Yu W-q, Jiang X-q, Zhang F-q, Xu L (2010) The effect of anatase TiO2 nanotube layers on MC3T3-E1 preosteoblast adhesion, proliferation, and differentiation. Journal of Biomedical Materials Research Part A 94A: 1012-1022.

71. Azhang Hamlekhan AB, Sweetu Patel, Dmitry Royhman, Christos Takoudis, Cortino Sukotjo, Mathew T Mathew, Tolou Shokuhfar (2014) Optimization of Anodization and Annealing Condition Enhances TiO2 Nanotubular Surface Hydrophilicity. TMS 2014 143rd Annual Meeting \& Exhibition, Annual Meeting Supplemental Proceedings: 221. 
72. Asthana A, Shokuhfar T, Gao Q, Heiden PA, Friedrich C, et al. (2010) A Real Time Observation of Phase Transition of Anatase TiO2 Nanotubes Into Rutile Nanoparticles by In-Situ Joule Heating Inside Transmission Electron Microscope. Advanced Science Letters 3: 557-562.

73. Arman Butt AH, Sweetu B Patel, Dmitry Royhman, Cortino Sukotjo, Mathew T Mathew, Tolou Shokuhfar, Christos Takoudis (2014) A Novel Investigation of the Formation of $\mathrm{TiO}_{2}$ Nanotubes on Thermally Formed Oxide of Ti-6Al-4V. Journal of Oral Implantology.

74. Azhang Hamlekhan AB, Sweetu Patel, Dmitry Royhman, Christos Takoudis, Cortino Sukotjo, Judy Yuan, Gregory Jursich, Mathew T. Mathew, William Hendrickson, Amarjit Virdi, Tolou Shokuhfar (2014) Fabrication of Anti-Aging TiO2 Nanotubes on Biomedical Ti Alloys. PLOS ONE.

75. Sweetu B Patel AH, Dmitry Royhman, Arman Butt, Judy Yuan, Tolou Shokuhfar, Cortino Sukotjo, Mathew T Mathew, Gregory M Jursich, Christos G Takoudis (2014) Enhancing Surface Characteristics of Ti-6Al-4V for Bio-implants Using Integrated Anodization and Thermal Oxidation. J Mater Chem B: DOI: 10.1039/C1033TB21731K.

76. Bai Y, Park S, Park HH, Lee MH, Bae TS, et al. (2011) The effect of annealing temperatures on surface properties, hydroxyapatite growth and cell behaviors of TiO2 nanotubes. Surface and Interface Analysis 43: 998-1005.

77. Zhao L, Mei S, Chu PK, Zhang Y, Wu Z (2010) The influence of hierarchical hybrid micro/nano-textured titanium surface with titania nanotubes on osteoblast functions. Biomaterials 31: 5072-5082.

78. Gao L, Feng B, Wang J, Lu X, Liu D, et al. (2009) Micro/Nanostructural Porous Surface on Titanium and Bioactivity. Journal of Biomedical Materials Research Part B-Applied Biomaterials 89B: 335-341.

79. Shin DH, Shokuhfar T, Choi CK, Lee S-H, Friedrich C (2011) Wettability changes of $\mathrm{TiO} 2$ nanotube surfaces. Nanotechnology 22.

80. Bauer S, Park J, von der Mark K, Schmuki P (2008) Improved attachment of mesenchymal stem cells on super-hydrophobic $\mathrm{TiO} 2$ nanotubes. Acta Biomaterialia 4: 1576-1582. 
81. Gong D, Grimes CA, Varghese OK, Hu WC, Singh RS, et al. (2001) Titanium oxide nanotube arrays prepared by anodic oxidation. Journal of Materials Research 16: 3331-3334.

82. Ghicov A, Tsuchiya H, Macak JM, Schmuki P (2005) Titanium oxide nanotubes prepared in phosphate electrolytes. Electrochemistry Communications 7: 505-509.

83. Bauer S, Kleber S, Schmuki P (2006) TiO2 nanotubes: Tailoring the geometry in H3PO4/HF electrolytes. Electrochemistry Communications 8: 1321-1325.

84. Sul YT, Johansson CB, Jeong Y, Albrektsson T (2001) The electrochemical oxide growth behaviour on titanium in acid and alkaline electrolytes. Medical Engineering \& Physics 23: 329-346.

85. Cai QY, Yang LX, Yu Y (2006) Investigations on the self-organized growth of TiO2 nanotube arrays by anodic oxidization. Thin Solid Films 515: 1802-1806.

86. Yasuda K, Schmuki P (2007) Control of morphology and composition of selforganized zirconium titanate nanotubes formed in (NH4)(2)SO4/NH4F electrolytes. Electrochimica Acta 52: 4053-4061.

87. Popat KC, Eltgroth M, La Tempa TJ, Grimes CA, Desai TA (2007) Titania nanotubes: A novel platform for drug-eluting coatings for medical implants? Small 3: 1878-1881.

88. Shokuhfar T (2010) Structural and surface property characterization of titanium dioxide nanotubes for orthopedic implants. Dissertation, Michigan Technological University.

89. Narayanan R, Kwon T-Y, Kim K-H (2009) TiO2 nanotubes from stirred glycerol/NH4F electrolyte: Roughness, wetting behavior and adhesion for implant applications. Materials Chemistry and Physics 117: 460-464.

90. Wan J, Yan X, Ding J, Wang M, Hu K (2009) Self-organized highly ordered TiO2 nanotubes in organic aqueous system. Materials Characterization 60: 1534-1540.

91. Moseke C, Hage F, Vorndran E, Gbureck U (2012) TiO2 nanotube arrays deposited on Ti substrate by anodic oxidation and their potential as a long-term drug delivery system for antimicrobial agents. Applied Surface Science 258: 53995404. 
92. Albu SP, Ghicov A, Macak JM, Schmuki P (2007) $250 \mathrm{mu}$ m long anodic TiO2 nanotubes with hexagonal self-ordering. Physica Status Solidi-Rapid Research Letters 1: R65-R67.

93. Zhang L, Shao J, Han Y (2011) Enhanced anodization growth of self-organized ZrO2 nanotubes on nanostructured zirconium. Surface \& Coatings Technology 205: 2876-2881.

94. Frandsen CJ, Brammer KS, Noh K, Connelly LS, Oh S, et al. (2011) Zirconium oxide nanotube surface prompts increased osteoblast functionality and mineralization. Materials Science \& Engineering C-Materials for Biological Applications 31: 1716-1722.

95. Saulacic N, Bosshardt DD, Bornstein MM, Berner S, Buser D (2012) Bone apposition to a titanium-zirconium alloy implant, as compared to two other titaniumcontaining implants. European Cells \& Materials 23: 273-288.

96. Mueller CK, Solcher P, Peisker A, Mtsariashvilli M, Schlegel KA, et al. (2013) Analysis of the influence of the macro- and microstructure of dental zirconium implants on osseointegration: a minipig study. Oral Surgery Oral Medicine Oral Pathology Oral Radiology 116: E1-E8.

97. P. Schmuki HT, Jan Macak and Luciano Taveira (2006) Formation of Self-Organized Zirconia Nanostructure. ECS Trans 1: 351-357.

98. Berger S, Faltenbacher J, Bauer S, Schmuki P (2008) Enhanced self-ordering of anodic $\mathrm{ZrO} 2$ nanotubes in inorganic and organic electrolytes using two-step anodization. Physica Status Solidi-Rapid Research Letters 2: 102-104.

99. Berger S, Jakubka F, Schmuki P (2008) Formation of hexagonally ordered nanoporous anodic zirconia. Electrochemistry Communications 10: 1916-1919.

100. Shin Y, Lee S (2009) A freestanding membrane of highly ordered anodic $\mathrm{ZrO} 2$ nanotube arrays. Nanotechnology 20.

101. Tsuchiya H, Macak JM, Ghicov A, Schmuki P (2006) Self-organization of anodic nanotubes on two size scales. Small 2: 888-891.

102. Feng XJ, Macak JM, Albu SP, Schmuki P (2008) Electrochemical formation of selforganized anodic nanotube coating on Ti-28Zr-8Nb biomedical alloy surface. Acta Biomaterialia 4: 318-323. 
103. Tsuchiya H, Akaki T, Nakata J, Terada D, Tsuji N, et al. (2009) Metallurgical aspects on the formation of self-organized anodic oxide nanotube layers. Electrochimica Acta 54: 5155-5162.

104. Nakada H, Numata Y, Sakae T, Okazaki Y, Tanimoto Y, et al. (2008) Comparison of Bone Mineral Density and Area of Newly Formed Bone Around Ti-15\%Zr$4 \% \mathrm{Nb}-4 \% \mathrm{Ta}$ Alloy and Ti-6\%Al-4\%V Alloy Implants. Journal of Hard Tissue Biology 17: 99-108.

105. Gulati K, Aw MS, Losic D (2011) Drug-eluting Ti wires with titania nanotube arrays for bone fixation and reduced bone infection. Nanoscale Research Letters 6.

106. Prato M, Kostarelos K, Bianco A (2008) Functionalized carbon nanotubes in drug design and discovery. Accounts of Chemical Research 41: 60-68.

107. Liu Z, Sun XM, Nakayama-Ratchford N, Dai HJ (2007) Supramolecular chemistry on water-soluble carbon nanotubes for drug loading and delivery. Acs Nano 1: $50-56$.

108. Liu Z, Chen K, Davis C, Sherlock S, Cao QZ, et al. (2008) Drug delivery with carbon nanotubes for in vivo cancer treatment. Cancer Research 68: 6652-6660.

109. Feazell RP, Nakayama-Ratchford N, Dai H, Lippard SJ (2007) Soluble singlewalled carbon nanotubes as longboat delivery systems for Platinum(IV) anticancer drug design. Journal of the American Chemical Society 129: 8438-+.

110. Kam NWS, Liu Z, Dai HJ (2005) Functionalization of carbon nanotubes via cleavable disulfide bonds for efficient intracellular delivery of siRNA and potent gene silencing. Journal of the American Chemical Society 127: 12492-12493.

111. Chen JY, Chen SY, Zhao XR, Kuznetsova LV, Wong SS, et al. (2008) Functionalized Single-Walled Carbon Nanotubes as Rationally Designed Vehicles for Tumor-Targeted Drug Delivery. Journal of the American Chemical Society 130: $16778-16785$.

112. Sun XM, Liu Z, Welsher K, Robinson JT, Goodwin A, et al. (2008) Nano-Graphene Oxide for Cellular Imaging and Drug Delivery. Nano Research 1: 203-212. 
113. Zhao XC, Liu RT (2012) Recent progress and perspectives on the toxicity of carbon nanotubes at organism, organ, cell, and biomacromolecule levels. Environment International 40: 244-255.

114. Poland CA, Duffin R, Kinloch I, Maynard A, Wallace WAH, et al. (2008) Carbon nanotubes introduced into the abdominal cavity of mice show asbestos-like pathogenicity in a pilot study. Nature Nanotechnology 3: 423-428.

115. Hu Y, Cai KY, Luo Z, Xu DW, Xie DC, et al. (2012) TiO2 nanotubes as drug nanoreservoirs for the regulation of mobility and differentiation of mesenchymal stem cells. Acta Biomaterialia 8: 439-448.

116. Bae IH, Yun KD, Kim HS, Jeong BC, Lim HP, et al. (2010) Anodic Oxidized Nanotubular Titanium Implants Enhance Bone Morphogenetic Protein-2 Delivery. Journal of Biomedical Materials Research Part B-Applied Biomaterials 93B: 484-491.

117. Aw MS, Addai-Mensah J, Losic D (2012) A multi-drug delivery system with sequential release using titania nanotube arrays. Chemical Communications 48 : 3348-3350.

118. Liang YQ, Cui ZD, Zhu SL, Yang XJ (2011) Characterization of self-organized $\mathrm{TiO} 2$ nanotubes on $\mathrm{Ti}-4 \mathrm{Zr}-22 \mathrm{Nb}-2 \mathrm{Sn}$ alloys and the application in drug delivery system. Journal of Materials Science-Materials in Medicine 22: 461-467.

119. Popat KC, Eltgroth M, LaTempa TJ, Grimes CA, Desai TA (2007) Decreased Staphylococcus epidermis adhesion and increased osteoblast functionality on antibiotic-loaded titania nanotubes. Biomaterials 28: 4880-4888.

120. Barao VAR, Mathew MT, Assuncao WG, Yuan JCC, Wimmer MA, et al. (2012) Stability of cp-Ti and Ti-6Al-4V alloy for dental implants as a function of saliva pH - an electrochemical study. Clinical Oral Implants Research 23: 1055-1062.

121. Yu WQ, Qiu J, Xu L, Zhang FQ (2009) Corrosion behaviors of TiO2 nanotube layers on titanium in Hank's solution. Biomedical Materials 4.

122. Kim H, Choi SH, Ryu JJ, Koh SY, Park JH, et al. (2008) The biocompatibility of SLA-treated titanium implants. Biomedical Materials 3.

123. Hu XX, Shen H, Shuai KG, Zhang EW, Bai YJ, et al. (2011) Surface bioactivity modification of titanium by $\mathrm{CO} 2$ plasma treatment and induction of 184 
hydroxyapatite: In vitro and in vivo studies. Applied Surface Science 257: 18131823.

124. Wilson CJ, Clegg RE, Leavesley DI, Pearcy MJ (2005) Mediation of biomaterialcell interactions by adsorbed proteins: A review. Tissue Engineering 11: 1-18.

125. Massia SP (1999) Cell-Extracellular Matrix Interactions Relevant to Vascular Tissue Engineering. Tissue Engineering of Vascular Prosthetic Grafts.

126. Webster TJ, Ergun C, Doremus RH, Siegel RW, Bizios R (2000) Specific proteins mediate enhanced osteoblast adhesion on nanophase ceramics. Journal of Biomedical Materials Research 51: 475-483.

127. Schneider G, Burridge K (1994) FORMATION OF FOCAL ADHESIONS BY OSTEOBLASTS ADHERING TO DIFFERENT SUBSTRATA. Experimental Cell Research 214: 264-269.

128. Steele JG, Dalton BA, Johnson G, Underwood PA (1993) POLYSTYRENE CHEMISTRY AFFECTS VITRONECTIN ACTIVITY - AN EXPLANATION FOR CELL ATTACHMENT TO TISSUE-CULTURE POLYSTYRENE BUT NOT TO UNMODIFIED POLYSTYRENE. Journal of Biomedical Materials Research 27: 927-940.

129. Dalton BA, McFarland CD, Gengenbach TR, Griesser HJ, Steele JG (1998) Polymer surface chemistry and bone cell migration. Journal of biomaterials science Polymer edition 9: 781-799.

130. Webb K, Hlady V, Tresco PA (2000) Relationships among cell attachment, spreading, cytoskeletal organization, and migration rate for anchorage-dependent cells on model surfaces. Journal of Biomedical Materials Research 49: 362-368.

131. Takebe J, Itoh S, Okada J, Ishibashi K (2000) Anodic oxidation and hydrothermal treatment of titanium results in a surface that causes increased attachment and altered cytoskeletal morphology of rat bone marrow stromal cells in vitro. Journal of Biomedical Materials Research 51: 398-407.

132. Liao HH, Andersson AS, Sutherland D, Petronis S, Kasemo B, et al. (2003) Response of rat osteoblast-like cells to microstructured model surfaces in vitro. Biomaterials 24: 649-654. 
133. Nourmohammadzadeh M, Lo JF, Bochenek M, Mendoza-Elias JE, Wang Q, et al. (2013) Microfluidic Array with Integrated Oxygenation Control for Real-Time Live-Cell Imaging: Effect of Hypoxia on Physiology of Microencapsulated Pancreatic Islets. Analytical Chemistry 85: 11240-11249.

134. Mazare A, Dilea M, Ionita D, Titorencu I, Trusca V, et al. (2012) Changing bioperformance of $\mathrm{TiO} 2$ amorphous nanotubes as an effect of inducing crystallinity. Bioelectrochemistry 87: 124-131.

135. Zhao LZ, Mei SL, Chu PK, Zhang YM, Wu ZF (2010) The influence of hierarchical hybrid micro/nano-textured titanium surface with titania nanotubes on osteoblast functions. Biomaterials 31: 5072-5082.

136. Gao L, Feng B, Wang JX, Lu X, Liu DL, et al. (2009) Micro/Nanostructural Porous Surface on Titanium and Bioactivity. Journal of Biomedical Materials Research Part B-Applied Biomaterials 89B: 335-341.

137. Shin DH, Shokuhfar T, Choi CK, Lee SH, Friedrich C (2011) Wettability changes of TiO2 nanotube surfaces. Nanotechnology 22.

138. Wan J, Yan X, Ding JJ, Wang M, Hu KC (2009) Self-organized highly ordered $\mathrm{TiO} 2$ nanotubes in organic aqueous system. Materials Characterization 60: 15341540.

139. Velten D, Biehl V, Aubertin F, Valeske B, Possart W, et al. (2002) Preparation of $\mathrm{TiO} 2$ layers on cp-Ti and Ti6A14V by thermal and anodic oxidation and by solgel coating techniques and their characterization. Journal of Biomedical Materials Research 59: 18-28.

140. Kluson P, Luskova H, Cerveny L, Klisakova J, Cajthaml T (2005) Partial photocatalytic oxidation of cyclopentene over titanium(IV) oxide. Journal of Molecular Catalysis A: Chemical 242: 62-67.

141. Woo S, Park J-H, Rhee CK, Lee J, Kim H (2012) Effect of thermal treatment on the aluminum hydroxide nanofibers synthesized by electrolysis of Al plates. Microelectronic Engineering 89: 89-91.

142. Nguyen QT, Kidder Jr JN, Ehrman SH (2002) Hybrid gas-to-particle conversion and chemical vapor deposition for the production of porous alumina films. Thin Solid Films 410: 42-52. 
143. Dai H-B, Ma G-L, Kang X-D, Wang P (2011) Hydrogen generation from coupling reactions of sodium borohydride and aluminum powder with aqueous solution of cobalt chloride. Catalysis Today 170: 50-55.

144. Riesgraf DA, May ML (1978) Infrared Spectra of Aluminum Hydroxide Chlorides. Appl Spectrosc 32: 362-366.

145. Dhonge BP, Mathews T, Sundari ST, Thinaharan C, Kamruddin M, et al. (2011) Spray pyrolytic deposition of transparent aluminum oxide (A12O3) films. Applied Surface Science 258: 1091-1096.

146. Yoshiya KERA STaKH (1967) Infrared Spectra of Surface V=O Bond of Vanadium Pentoxide. Short Communication 40: 1.

147. Ercan B, Taylor E, Alpaslan E, Webster TJ (2011) Diameter of titanium nanotubes influences anti-bacterial efficacy. Nanotechnology 22.

148. Masahashi N, Mizukoshi Y, Semboshi S, Ohmura K, Hanada S (2012) Photoinduced properties of anodic oxide films on Ti6Al4V. Thin Solid Films 520: 4956-4964.

149. Jimbo R, Sawase T, Baba K, Kurogi T, Shibata Y, et al. (2008) Enhanced initial cell responses to chemically modified anodized titanium. Clinical Implant Dentistry and Related Research 10: 55-61.

150. Oh S, Brammer KS, Li YSJ, Teng DY, Engler AJ, et al. (2009) Reply to von der Mark et al.: Looking further into the effects of nanotube dimension on stem cell fate. Proceedings of the National Academy of Sciences of the United States of America 106: E61-E61.

151. Brisman DL, Brisman AS, Moses MS (2001) Implant failures associated with asymptomatic endodontically treated teeth. Journal of the American Dental Association 132: 191-195.

152. Saeedeh Rahbarpour MP (2011) Silver-Rutile Schottky Diode Fabricated on Oxidized Titanium Foil. IOP Conference Series: Materials Science and Engineering 17: 012016.

153. Doroudian G, Curtis MW, Gang A, Russell B (2013) Cyclic strain dominates over microtopography in regulating cytoskeletal and focal adhesion remodeling of 
human mesenchymal stem cells. Biochemical and Biophysical Research Communications 430: 1040-1046.

154. Tolou Shokuhfar SS-R, Cortino Sukotjoc, Alexander L. Yarin (2013) Intercalation of anti-inflammatory drug molecules within TiO2 nanotubes. RSC Adv 3: 1738017386.

155. Gulati K, Ramakrishnan S, Aw MS, Atkins GJ, Findlay DM, et al. (2012) Biocompatible polymer coating of titania nanotube arrays for improved drug elution and osteoblast adhesion. Acta Biomaterialia 8: 449-456.

156. Li Z, Ning C, Ding D, Liu H, Huang L (2012) Biological Properties of Ti-Nb-Zr-O Nanostructures Grown on Ti35Nb5Zr Alloy. Journal of Nanomaterials.

157. Lai M, Cai KY, Zhao L, Chen XY, Hou YH, et al. (2011) Surface Functionalization of TiO2 Nanotubes with Bone Morphogenetic Protein 2 and Its Synergistic Effect on the Differentiation of Mesenchymal Stem Cells. Biomacromolecules 12: 10971105.

158. Hu Y, Cai K, Luo Z, Xu D, Xie D, et al. (2012) TiO2 nanotubes as drug nanoreservoirs for the regulation of mobility and differentiation of mesenchymal stem cells. Acta Biomaterialia 8: 439-448.

159. Kazemzadeh-Narbat M, Lai BFL, Ding C, Kizhakkedathu JN, Hancock REW, et al. (2013) Multilayered coating on titanium for controlled release of antimicrobial peptides for the prevention of implant-associated infections. Biomaterials 34 : 5969-5977.

160. Moom Sinn Aw KG, Dusan Losic (2011) Controlling Drug Release from Titania Nanotube Arrays Using Polymer Nanocarriers and Biopolymer Coating. Journal of Biomaterials and Nanobiotechnology 2: 477-484.

161. Aw MS, Simovic S, Addai-Mensah J, Losic D (2011) Polymeric micelles in porous and nanotubular implants as a new system for extended delivery of poorly soluble drugs. Journal of Materials Chemistry 21: 7082-7089.

162. Rajesh P, Mohan N, Yokogawa Y, Varma H (2013) Pulsed laser deposition of hydroxyapatite on nanostructured titanium towards drug eluting implants. Materials Science \& Engineering C-Materials for Biological Applications 33: 2899-2904. 
163. Gulati K, Aw MS, Losic D (2012) Nanoengineered drug-releasing Ti wires as an alternative for local delivery of chemotherapeutics in the brain. International Journal of Nanomedicine 7: 2069-2076.

164. Aw MS, Khalid KA, Gulati K, Atkins GJ, Pivonka P, et al. (2012) Characterization of drug-release kinetics in trabecular bone from titania nanotube implants. International Journal of Nanomedicine 7: 4883-4892.

165. Bae I-H, Yun K-D, Kim H-S, Jeong B-C, Lim H-P, et al. (2010) Anodic Oxidized Nanotubular Titanium Implants Enhance Bone Morphogenetic Protein-2 Delivery. Journal of Biomedical Materials Research Part B-Applied Biomaterials 93B: 484-491.

166. Lai S, Zhang W, Liu F, Wu C, Zeng D, et al. (2013) TiO2 Nanotubes as Animal Drug Delivery System and In Vitro Controlled Release. Journal of Nanoscience and Nanotechnology 13: 91-97.

167. Peng L, Mendelsohn AD, LaTempa TJ, Yoriya S, Grimes CA, et al. (2009) LongTerm Small Molecule and Protein Elution from TiO2 Nanotubes. Nano Letters 9: 1932-1936.

168. Bazilevsky AV, Sun KX, Yarin AL, Megaridis CM (2007) Selective intercalation of polymers in carbon nanotubes. Langmuir 23: 7451-7455.

169. Bazilevsky AV, Sun K, Yarin AL, Megaridis CM (2008) Room-temperature, openair, wet intercalation of liquids, surfactants, polymers and nanoparticles within nanotubes and microchannels. Journal of Materials Chemistry 18: 696-702.

170. Sinha-Ray S, Sahu RP, Yarin AL (2011) Nano-encapsulated smart tunable phase change materials. Soft Matter 7: 8823-8827.

171. N. Adya Ma, T. Ravindranath, A. Mubeen, B. Saluja. (2005) Corrosion in titanium dental implants: Literature review. The Journal of Indian Prosthodontic Society 5: 6.

172. Dong H, Li XY (2000) Oxygen boost diffusion for the deep-case hardening of titanium alloys. Materials Science and Engineering: A 280: 303-310.

173. MacDonald DE, Rapuano BE, Deo N, Stranick M, Somasundaran P, et al. (2004) Thermal and chemical modification of titanium-aluminum-vanadium implant 
materials: effects on surface properties, glycoprotein adsorption, and MG63 cell attachment. Biomaterials 25: 3135-3146.

174. Donos N, Hamlet S, Lang NP, Salvi GE, Huynh-Ba G, et al. (2011) Gene expression profile of osseointegration of a hydrophilic compared with a hydrophobic microrough implant surface. Clinical Oral Implants Research 22: 365-372.

175. Park JH, Olivares-Navarrete R, Baier RE, Meyer AE, Tannenbaum R, et al. (2012) Effect of cleaning and sterilization on titanium implant surface properties and cellular response. Acta Biomaterialia 8: 1966-1975.

176. Rinner M, Gerlach J, Ensinger W (2000) Formation of titanium oxide films on titanium and Ti6A14V by O2-plasma immersion ion implantation. Surface and Coatings Technology 132: 111-116.

177. Ogawa T, Sukotjo C, Nishimura I (2002) Modulated bone matrix-related gene expression is associated with differences in interfacial strength of different implant surface roughness. Journal of Prosthodontics 11: 241-247.

178. Boyan BD, Lohmann CH, Dean DD, Sylvia VL, Cochran DL, et al. (2001) MECHANISMS INVOLVED IN OSTEOBLAST RESPONSE TO IMPLANT SURFACE MORPHOLOGY. Annual Review of Materials Research 31: 357-371.

179. de Oliveira PT, Zalzal SF, Beloti MM, Rosa AL, Nanci A (2007) Enhancement of in vitro osteogenesis on titanium by chemically produced nanotopography. Journal of Biomedical Materials Research Part A 80A: 554-564.

180. Joos U, Meyer U (2006) New paradigm in implant osseointegration. Head \& Face Medicine 2: 19.

181. Czarnowska E, Wierzchoń T, Maranda-Niedbała A (1999) Properties of the surface layers on titanium alloy and their biocompatibility in in vitro tests. Journal of Materials Processing Technology 92-93: 190-194.

182. Ponsonnet L, Reybier K, Jaffrezic N, Comte V, Lagneau C, et al. (2003) Relationship between surface properties (roughness, wettability) of titanium and titanium alloys and cell behaviour. Materials Science and Engineering: C 23: 551560.

183. Feng B, Weng J, Yang BC, Qu SX, Zhang XD (2003) Characterization of surface oxide films on titanium and adhesion of osteoblast. Biomaterials 24: 4663-4670. 
184. Khan MR, Donos N, Salih V, Brett PM (2012) The enhanced modulation of key bone matrix components by modified Titanium implant surfaces. Bone 50: 1-8.

185. Pakhlov E, Voronin E, Borysenko M, Yurchenko G (2000) Study of Water, Methanol And Isopropanol Adsorption on The Surface of Titanosilicas of Different Structure. Journal of Thermal Analysis and Calorimetry 62: 395-399.

186. Kasuga T, Kondo H, Nogami M (2002) Apatite formation on $\mathrm{TiO} 2$ in simulated body fluid. Journal of Crystal Growth 235: 235-240.

187. Miyauchi M, Kieda N, Hishita S, Mitsuhashi T, Nakajima A, et al. (2002) Reversible wettability control of $\mathrm{TiO} 2$ surface by light irradiation. Surface Science 511: 401-407.

188. George SM (2010) Atomic Layer Deposition: An Overview. Chemical Reviews 110: 21 .

189. K. Overhage QT, G. Jursich, and C. G. Takoudis (2011) Selective Atomic Layer Deposition (SALD) of Titanium Dioxide on Silicon and Copper Patterned Substrate. Journal of Undergraduate Research 4.

190. Ivanoff C-J, Widmark G, Hallgren C, Sennerby L, Wennerberg A (2001) Histologic evaluation of the bone integration of $\mathrm{TiO}_{2}$ blasted and turned titanium microimplants in humans. Clinical Oral Implants Research 12: 128-134.

191. Kumar S, Sankara Narayanan TSN, Ganesh Sundara Raman S, Seshadri SK (2010) Thermal oxidation of Ti6A14V alloy: Microstructural and electrochemical characterization. Materials Chemistry and Physics 119: 337-346.

192. Hanawa T (2011) A comprehensive review of techniques for biofunctionalization of titanium. J Periodontal Implant Sci 41: 263-272.

193. Lee S-M, Pippel E, Knez M (2011) Metal Infiltration into Biomaterials by ALD and CVD: A Comparative Study. Chemphyschem 12: 791-798.

194. Tao Q, Overhage K, Jursich G, Takoudis C (2012) On the initial growth of atomic layer deposited TiO2 films on silicon and copper surfaces. Thin Solid Films 520: 6752-6756.

195. Takoudis XSaCG (2007) Effect of NH3 on the low pressure chemical vapor deposition of $\mathrm{TiO} 2$ film at low temperature using tetrakis(diethylamino)titanium and oxygen. Journal of vacuum Science \& Technology A 25. 
196. Rosa C (1970) Oxygen diffusion in alpha and beta titanium in the temperature range of $932^{\circ}$ to $1142^{\circ} \mathrm{C}$. Metallurgical Transactions 1: 2517-2522.

197. Motte F, Coddet C, Sarrazin P, Azzopardi M, Besson J (1976) A comparative study of the oxidation with water vapor of pure titanium and of Ti-6Al-4V. Oxidation of Metals 10: 113-126.

198. D. David and G. Beranger aEAG (1983) A Study of the Diffusion of Oxygen in $\alpha$ Titanium Oxidized in the Temperature Range $460-700^{\circ} \mathrm{C}$. Journal of Electrochemical Society: Solid-State Science And Technology: 4.

199. J.W. Rogers J, K.L. Erickson, D.N. Belton, R.W. Springer, T.N. Taylor And J.G. Beery (1988-1989) Low Temperature Diffusion Of Oxygen In Titanium And Titanium Oxide Films. Applied Surface Science 35: 16.

200. Liu Z, Welsch G (1988) Literature Survey on Diffusivities of Oxygen, Aluminum, and Vanadium in Alpha Titanium, Beta Titanium, and in Rutile. Metallurgical Transactions A 19: 1121-1125.

201. Padma R, Ramkumar K, Satyam M (1988) Growth of titanium oxide overlayers by thermal oxidation of titanium. Journal of Materials Science 23: 1591-1597.

202. Kusabiraki K, Kuroda N, Motohira I, Ooka T (1997) High-temperature oxidation of pure titanium in $\mathrm{CO} 2$ and Ar-10\%CO2 atmospheres. Oxidation of Metals 48: 289302.

203. García-Alonso MC, Saldaña L, Vallés G, González-Carrasco JL, González-Cabrero J, et al. (2003) In vitro corrosion behaviour and osteoblast response of thermally oxidised Ti6Al4V alloy. Biomaterials 24: 19-26.

204. J. H. Park ZS, R. Olivares-Navarrete, B. D. Boyan and R. Tannenbaum (2011) Langmuir 27 5985.: 10.

205. Oh S, Brammer KS, Li YSJ, Teng D, Engler AJ, et al. (2009) Stem cell fate dictated solely by altered nanotube dimension. Proceedings of the National Academy of Sciences.

206. H Yin HLaWZS (2010) The large diameter and fast growth of self-organized TiO2 nanotube arrays achieved via electrochemical anodization. Nanotechnology 21: 8.

207. Vasilev K, Poh Z, Kant K, Chan J, Michelmore A, et al. (2010) Tailoring the surface functionalities of titania nanotube arrays. Biomaterials 31: 532-540. 
208. Sulka GD, Kapusta-Kołodziej J, Brzózka A, Jaskuła M (2010) Fabrication of nanoporous $\mathrm{TiO} 2$ by electrochemical anodization. Electrochimica Acta 55: 43594367.

209. Okada M, Tajima K, Yamada Y, Yoshimura K (2012) Self-Organized Formation of Short TiO2 Nanotube Arrays By Complete Anodization of Ti Thin Films. Physics Procedia 32: 714-718.

210. Prakasam HE, Shankar K, Paulose M, Varghese OK, Grimes CA (2007) A New Benchmark for TiO2 Nanotube Array Growth by Anodization. The Journal of Physical Chemistry C 111: 7235-7241.

211. Paulose M, Prakasam HE, Varghese OK, Peng L, Popat KC, et al. (2007) TiO2 Nanotube Arrays of $1000 \mu \mathrm{m}$ Length by Anodization of Titanium Foil: Phenol Red Diffusion. The Journal of Physical Chemistry C 111: 14992-14997.

212. Guohua L, Hoivik N, Kaiying W, Jakobsen H. Free-standing TiO $<\inf >2</$ inf $>$ nanotube membranes from electrochemical anodization; 2011 15-18 Aug. 2011. pp. 1011-1015.

213. Zihan Poh KV, Krishna Kant Reza Emamali Sabzi and Dusan Losie Synthesis, Nanostructure and Crystallization of Titania Nanotube Arrays Fabricated by Electrochemical Anodization. Ian Work Research Institue and Mawson Institue. pp. 4.

214. Lin J, Chen J, Chen X (2010) Facile fabrication of free-standing TiO2 nanotube membranes with both ends open via self-detaching anodization. Electrochemistry Communications 12: 1062-1065.

215. Albu SP, Schmuki P (2013) Influence of anodization parameters on the expansion factor of TiO2 nanotubes. Electrochimica Acta 91: 90-95.

216. Panaitescu E (2009) Titanium oxide nanotubes: synthesis, properties and applications for solar energy harvesting: Northeastern University.

217. Dawei Gong CAG, Oomman K. Varghese, Wenchong Hu, R. S. Singh, Zhi Chen, and Elizabeth C. Dickey (2001) Titanium oxide nanotube arrays prepared by anodic oxidation. Journal of Materials Research 16: 4.

218. Shin DH, Shokuhfar T, Choi CK, Lee S, Friedrich C (2011) Wettability changes of TiO2 nanotube surfaces. Nanotechnology 22: 315704. 
219. Das K, Bose S, Bandyopadhyay A (2009) TiO2 nanotubes on Ti: Influence of nanoscale morphology on bone cell-materials interaction. Journal of Biomedical Materials Research Part A 90A: 225-237.

220. Sul YT (2010) Electrochemical growth behavior, surface properties, and enhanced in vivo bone response of $\mathrm{TiO} 2$ nanotubes on microstructured surfaces of blasted, screw-shaped titanium implants. International journal of nanomedicine 5: 87-100.

221. Le Guehennec L, Lopez-Heredia M-A, Enkel B, Weiss P, Amouriq Y, et al. (2008) Osteoblastic cell behaviour on different titanium implant surfaces. Acta Biomaterialia 4: 535-543.

222. Macak JM, Tsuchiya H, Taveira L, Ghicov A, Schmuki P (2005) Self-organized nanotubular oxide layers on Ti-6Al-7Nb and Ti-6Al-4V formed by anodization in NH4F solutions. Journal of Biomedical Materials Research Part A 75A: 928-933.

223. Macak JM, Tsuchiya H, Ghicov A, Yasuda K, Hahn R, et al. (2007) TiO2 nanotubes: Self-organized electrochemical formation, properties and applications. Current Opinion in Solid State and Materials Science 11:3-18.

224. Saldaña L, Barranco V, González-Carrasco JL, Rodríguez M, Munuera L, et al. (2007) Thermal oxidation enhances early interactions between human osteoblasts and alumina blasted Ti6A14V alloy. Journal of Biomedical Materials Research Part A 81A: 334-346.

225. Osamu Tonomura, Tomoko Sekiguchi, Naomi Inada, Tomoyuki Hamada, and HM, et al. (2012) Band Engineering of Ru/Rutile-TiO2/Ru Capacitors by Doping Cobalt to Suppress Leakage Current. Journal of The Electrochemical Society 159: 5.

226. Weeranut Kaewwiset WOaPL (2008) Effect of Annealed Temperatures on the Morphology of TiO2 Films. Nat Sci 42: 6.

227. Zeitler VA, Brown CA (1957) The Infrared Spectra of Some Ti-O-Si, Ti-O-Ti and Si-O-Si Compounds. The Journal of Physical Chemistry 61: 1174-1177.

228. Bagheri S, Shameli K, Abd Hamid SB (2013) Synthesis and Characterization of Anatase Titanium Dioxide Nanoparticles Using Egg White Solution via Sol-Gel Method. Journal of Chemistry 2013: 5. 
229. McCarthy SA, Davies G-L, Gun'ko YK (2012) Preparation of multifunctional nanoparticles and their assemblies. Nat Protocols 7: 1677-1693.

230. Pankewitz T, Lagutschenkov A, Niedner-Schatteburg G, Xantheas SS, Lee Y-T (2007) Infrared spectrum of $\mathrm{NH}[\mathrm{sub} 4][\sup +](\mathrm{H}[\mathrm{sub} 2] \mathrm{O})$ : Evidence for mode specific fragmentation. The Journal of Chemical Physics 126: 074307-074314.

231. Shen YF, Suib SL, Deeba M, Koermer GS (1994) Luminescence and IR Characterization of Acid Sites on Alumina. Journal of Catalysis 146: 483-490.

232. Bezrodna T, Puchkovska G, Shymanovska V, Baran J, Ratajczak H (2004) IRanalysis of $\mathrm{H}$-bonded $\mathrm{H} 2 \mathrm{O}$ on the pure $\mathrm{TiO} 2$ surface. Journal of Molecular Structure 700: 175-181.

233. Nolan NT, Seery MK, Pillai SC (2009) Spectroscopic Investigation of the Anataseto-Rutile Transformation of Sol-Gel-Synthesized TiO2 Photocatalysts. The Journal of Physical Chemistry C 113: 16151-16157.

234. Golhotra P (2010) Carbon dioxide adsorption on nanomaterials: University of Iowa. $165 \mathrm{p}$.

235. Rubasinghege GRS (2011) Chemical and photochemical reactions on mineral oxide surfaces in gaseous and liquid phases: environmental implications of fate, transport and climate impacts of mineral dust aerosaol: University of Iowa.

236. . Principles of IR Spectroscopy. pp. 31.

237. Fang WQ, Gong X-Q, Yang HG (2011) On the Unusual Properties of Anatase TiO2 Exposed by Highly Reactive Facets. The Journal of Physical Chemistry Letters 2: 725-734.

238. Hua Gui Yang CHS, Shi Zhang Qiao, Jin Zou, Gang Liu, Sean Campbell Smith, Hui Ming Cheng, Gao Qing $\mathrm{Lu}$ (2008) Anatase $\mathrm{TiO}_{2}$ single crystals with a large percentage of reactive facets. Nature 453: 4 .

239. Cho CH, Han MH, Kim DH, Kim DK (2005) Morphology evolution of anatase TiO2 nanocrystals under a hydrothermal condition $(\mathrm{pH} \& \# \mathrm{xa} 0 ;=\& \# \mathrm{xa} 0 ; 9.5)$ and their ultra-high photo-catalytic activity. Materials Chemistry and Physics 92: 104111. 
240. Lu J, Dai Y, Jin H, Huang B (2011) Effective increasing of optical absorption and energy conversion efficiency of anatase $\mathrm{TiO} 2$ nanocrystals by hydrogenation. Physical Chemistry Chemical Physics 13: 18063-18068.

241. Nakata K, Fujishima A (2012) TiO2 photocatalysis: Design and applications. Journal of Photochemistry and Photobiology C: Photochemistry Reviews 13: 169189.

242. Ji-Ho Park D-YLaK-NK. CHARACTERIZATION OF THE SURFACE OXIDE

ON THERMALLY OXIDIZED Ti6Al4V ALLOY

FOR IMPROVEMENT OF BIOACTIVITY; 2004.

243. Marmur A (2003) Wetting on Hydrophobic Rough Surfaces: To Be Heterogeneous or Not To Be? Langmuir 19: 8343-8348.

244. Verplanck N, Coffinier Y, Thomy V, Boukherroub R (2007) Wettability Switching Techniques on Superhydrophobic Surfaces. Nanoscale Research Letters 2: 577 596.

245. Neumann AW, Good RJ (1972) Thermodynamics of contact angles. I. Heterogeneous solid surfaces. Journal of Colloid and Interface Science 38: 341358.

246. Giljean S, Bigerelle M, Anselme K, Haidara H (2011) New insights on contact angle/roughness dependence on high surface energy materials. Applied Surface Science 257: 9631-9638.

247. Lee YYaTR (2013) Contact Angle and Wetting Properties. springer series in surface science 51.

248. Cassie ABD, Baxter S (1944) Wettability of porous surfaces. Transactions of the Faraday Society 40: 546-551.

249. young T Biomaterials. surfaces: contact angle phenomena and wetting.

250. Oliver JF, Huh C, Mason SG (1976) The Apparent Contact Angle of Liquids on Finely-Grooved Solid Surfaces-A SEM Study. The Journal of Adhesion 8: 223234.

251. Rupp F, Scheideler L, Olshanska N, de Wild M, Wieland M, et al. (2006) Enhancing surface free energy and hydrophilicity through chemical modification of 196 
microstructured titanium implant surfaces. Journal of Biomedical Materials Research Part A 76A: 323-334.

252. Oliver JF, Mason SG (1980) \{L\}iquid spreading on rough metal surfaces. J Mater Sci 15: 431-437.

253. Massaro C, Rotolo P, De Riccardis F, Milella E, Napoli A, et al. (2002) Comparative investigation of the surface properties of commercial titanium dental implants. Part I: chemical composition. Journal of Materials Science: Materials in Medicine 13: 535-548.

254. Tsuchiya H, Macak JM, Ghicov A, Tang YC, Fujimoto S, et al. (2006) Nanotube oxide coating on Ti-29Nb-13Ta-4.6Zr alloy prepared by self-organizing anodization. Electrochimica Acta 52: 94-101.

255. Luo B, Yang H, Liu S, Fu W, Sun P, et al. (2008) Fabrication and characterization of self-organized mixed oxide nanotube arrays by electrochemical anodization of Ti-6Al-4V alloy. Materials Letters 62: 4512-4515.

256. Jakubowicz J, Adamek G (2009) Preparation and properties of mechanically alloyed and electrochemically etched porous Ti-6Al-4V. Electrochemistry Communications 11: 1772-1775.

257. Yao C, Perla V, McKenzie JL, Slamovich EB, Webster TJ (2005) Anodized Ti and Ti6A14V Possessing Nanometer Surface Features Enhances Osteoblast Adhesion. Journal of Biomedical Nanotechnology 1: 68-73.

258. Matykina E, Conde A, de Damborenea J, Marero DMy, Arenas MA (2011) Growth of TiO2-based nanotubes on Ti-6Al-4V alloy. Electrochimica Acta 56: 92099218.

259. Matykina E, Hernandez-López JM, Conde A, Domingo C, de Damborenea JJ, et al. (2011) Morphologies of nanostructured TiO2 doped with F on Ti-6Al-4V alloy. Electrochimica Acta 56: 2221-2229.

260. Xie J, Luan BL (2008) Nanometer-scale surface modification of Ti6Al4V alloy for orthopedic applications. Journal of Biomedical Materials Research Part A 84A: 63-72. 
261. Schmuki AGaP (2009) Self-ordering electrochemistry: a review on growth and functionality of $\mathrm{TiO}_{2}$ nanotubes and other self-aligned $\mathrm{MO}_{\mathrm{x}}$ structures. The Royal Society of Chemistry: 18.

262. G. Ketteler SY, H. Bluhm, K. Andersson, D.E. Starr, D. F. Ogletree, H. Ogasawara, A. Nilsson, and M. Salmeron (2007) The Journal of Physical Chemistry C 111.

263. Ogawa T, Sukotjo C, Nishimura I (2002) Modulated bone matrix-related gene expression is associated with differences in interfacial strength of different implant surface roughness. Journal of prosthodontics : official journal of the American College of Prosthodontists 11: 241-247.

264. Adya N AM, Ravindranath T, Mubeen a, Saluja B (2005) Corrosion in titanium dental implants: literature review. J Indian Prosthodont Soc 5: 126.

265. Dong H, Li XY (2000) Oxygen boost diffusion for the deep-case hardening of titanium alloys. Materials Science and Engineering a-Structural Materials Properties Microstructure and Processing 280: 303-310.

266. Rinner M, Gerlach J, Ensinger W (2000) Formation of titanium oxide films on titanium and Ti6A14V by O-2-plasma immersion ion implantation. Surface \& Coatings Technology 132: 111-116.

267. MacDonald DE, Rapuano BE, Deo N, Stranick M, Somasundaran P, et al. (2004) Thermal and chemical modification of titanium-aluminum-vanadium implant materials: effects on surface properties, glycoprotein adsorption, and MG63 cell attachment. Biomaterials 25: 3135-3146.

268. Park JH, Schwartz Z, Olivares-Navarrete R, Boyan BD, Tannenbaum R (2011) Enhancement of Surface Wettability via the Modification of Microtextured Titanium Implant Surfaces with Polyelectrolytes. Langmuir 27: 5976-5985.

269. Boyan BD, Lohmann CH, Dean DD, Sylvia VL, Cochran DL, et al. (2001) Mechanisms involved in osteoblast response to implant surface morphology. Annual Review of Materials Research 31: 357-371.

270. Joos U, Meyer U (2006) New paradigm in implant osseointegration. Head \& face medicine 2: 19-19. 
271. Czarnowska E, Wierzchon T, Maranda-Niedbala A (1999) Properties of the surface layers on titanium alloy and their biocompatibility in in vitro tests. Journal of Materials Processing Technology 93: 190-194.

272. Pakhlov EM, Voronin EF, Borysenko MV, Yurchenko GR (2000) Study of water, methanol and isopropanol adsorption on the surface of titanosilicas of different structure. Journal of Thermal Analysis and Calorimetry 62: 395-399.

273. Kasuga T, Kondo H, Nogami M (2002) Apatite formation on $\mathrm{TiO} 2$ in simulated body fluid. Journal of Crystal Growth 235: 235-240.

274. Miyauchi M, Kieda N, Hishita S, Mitsuhashi T, Nakajima A, et al. (2002) Reversible wettability control of $\mathrm{TiO} 2$ surface by light irradiation. Surface Science 511: 401-407.

275. Ponsonnet L, Reybier K, Jaffrezic N, Comte V, Lagneau C, et al. (2003) Relationship between surface properties (roughness, wettability) of titanium and titanium alloys and cell behaviour. Materials Science \& Engineering CBiomimetic and Supramolecular Systems 23: 551-560.

276. Elias CN, Oshida Y, Cavalcanti Lima JH, Muller CA (2008) Relationship between surface properties (roughness, wettability and morphology) of titanium and dental implant removal torque. Journal of the Mechanical Behavior of Biomedical Materials 1: 234-242.

277. Wall I, Donos N, Carlqvist K, Jones F, Brett P (2009) Modified titanium surfaces promote accelerated osteogenic differentiation of mesenchymal stromal cells in vitro. Bone 45: 17-26.

278. Miyauchi Y, Ninomiya K, Miyamoto H, Sakamoto A, Iwasaki R, et al. (2010) The Blimp1-Bcl6 axis is critical to regulate osteoclast differentiation and bone homeostasis. Journal of Experimental Medicine 207: 751-762.

279. Vlacic-Zischke J, Hamlet SM, Frus T, Tonetti MS, Ivanovski S (2011) The influence of surface microroughness and hydrophilicity of titanium on the upregulation of TGF beta/BMP signalling in osteoblasts. Biomaterials 32: 665-671.

280. Keshmiri M, Troczynski T (2003) Apatite formation on $\mathrm{TiO} 2$ anatase microspheres. Journal of Non-Crystalline Solids 324: 289-294. 
281. Amin MS, Randeniya LK, Bendavid A, Martin PJ, Preston EW (2010) Apatite formation from simulated body fluid on various phases of $\mathrm{TiO} 2$ thin films prepared by filtered cathodic vacuum arc deposition. Thin Solid Films 519: 13001306.

282. Hanawa T (2011) A comprehensive review of techniques for biofunctionalization of titanium. Journal of periodontal \& implant science 41: 263-272.

283. Garcia-Alonso MC, Saldana L, Valles G, Gonzalez-Carrasco JL, Gonzalez-Cabrero $\mathrm{J}$, et al. (2003) In vitro corrosion behaviour and osteoblast response of thermally oxidised Ti6Al4V alloy. Biomaterials 24: 19-26.

284. Alves C, Neto C, Morais GHS, da Silva CF, Hajek V (2005) Nitriding of titanium disks and industrial dental implants using hollow cathode discharge. Surface \& Coatings Technology 194: 196-202.

285. Guerra Neto CLB, da Silva MAM, Alves C, Jr. (2009) Osseointegration evaluation of plasma nitrided titanium implants. Surface Engineering 25: 434-439.

286. Clem WC, Konovalov VV, Chowdhury S, Vohra YK, Catledge SA, et al. (2006) Mesenchymal stem cell adhesion and spreading on microwave plasma-nitrided titanium alloy. Journal of Biomedical Materials Research Part A 76A: 279-287.

287. KC K (2008) Atomic Layer Deposition of Hafnium Oxide on Silicon and Polymer Fibers at Temperatures below $100 \circ \mathrm{C}$. J Undergrad Res 1: 5 .

288. George SM (2010) Atomic Layer Deposition: An Overview. Chemical Reviews 110: 111-131.

289. K. O (2010) Selective Atomic Layer Deposition ( SALD ) of Titanium Dioxide on Silicon and Copper Patterned Substrates. 1: 8.

290. Ivanoff CJ, Hallgren C, Widmark G, Sennerby L, Wennerberg A (2001) Histologic evaluation of the bone integration of $\mathrm{TiO} 2$ blasted and turned titanium microimplants in humans. Clinical Oral Implants Research 12: 128-134.

291. Kumar S, Narayanan TSNS, Raman SGS, Seshadri SK (2010) Thermal oxidation of CP Ti - An electrochemical and structural characterization. Materials Characterization 61: 589-597. 
292. Tao QTQ JG, Takoudis C (2010) Structural and dielectric characterization of atomic layer deposited $\mathrm{HfO} 2$ and $\mathrm{TiO} 2$ as promising gate oxides. Adv Semicond Manuf Conf ASMC 2010 IEEESEMI: 17-22.

293. Tao Q KA, Singh M, Jursich G, Takoudis CG (2011) Atomic Layer Deposition of

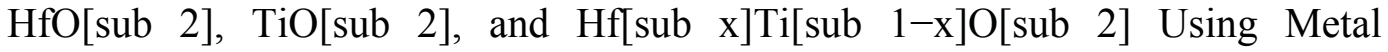
(Diethylamino) Precursors and H[sub 2]O. J Electrochem Soc 158: G27.

294. Selvaraj SK, Jursich G, Takoudis CG (2013) Design and implementation of a novel portable atomic layer deposition/chemical vapor deposition hybrid reactor. Review of Scientific Instruments 84.

295. Song X, Takoudis CG (2007) Effect of NH3 on the low pressure chemical vapor deposition of $\mathrm{TiO} 2$ film at low temperature using tetrakis(diethylamino)titanium and oxygen. Journal of Vacuum Science \& Technology A 25: 360-367.

296. CJ R (1970) Oxygen Diffusion in Alpha and Beta Titanium in the Temperature Range of $932 \sim$ to $1142 \sim$. 1: 2517-2522.

297. Motte F CC, Sarrazin P, Azzopardi M, Besson J (1976) A Comparative Study of the Oxidation with Water Vapor of Pure Titanium and of Ti-6AI-4V. 10.

298. Range T GE A Study of the Diffusion of Oxygen in c -Titanium Oxidized in the Temperature Range 460 176. 130.

299. J.W. Rogers J, K. L. Erickson, D. N. Belton, R. W. Springer, T. N. Taylor JGB (1988) Low temperature diffusion and titanium oxide films. 35.

300. (1988) Titanium B Communications 19: 1121-1125.

301. Padma R, Ramkumar K, Satyam M (1988) GROWTH OF TITANIUM-OXIDE OVERLAYERS BY THERMAL-OXIDATION OF TITANIUM. Journal of Materials Science 23: 1591-1597.

302. Herman GS, Dohnalek Z, Ruzycki N, Diebold U (2003) Experimental investigation of the interaction of water and methanol with anatase-TiO2(101). Journal of Physical Chemistry B 107: 2788-2795.

303. Zhang Z, Bondarchuk O, Kay BD, White JM, Dohnalek Z (2006) Imaging water dissociation on $\mathrm{TiO}$ (110): Evidence for inequivalent geminate $\mathrm{OH}$ groups. Journal of Physical Chemistry B 110: 21840-21845. 
304. E P (2009) Titanium oxide nanotubes: synthesis, properties and applications for solar energy harvesting.

305. Kowalski D, Kim D, Schmuki P (2013) TiO2 nanotubes, nanochannels and mesosponge: Self-organized formation and applications. Nano Today 8: 235-264.

306. Chen B, Hou J, Lu K (2013) Formation Mechanism of TiO2 Nanotubes and Their Applications in Photoelectrochemical Water Splitting and Supercapacitors. Langmuir 29: 5911-5919.

307. Berger S, Albu SP, Schmidt-Stein F, Hildebrand H, Schmuki P, et al. (2011) The origin for tubular growth of $\mathrm{TiO} 2$ nanotubes: A fluoride rich layer between tubewalls. Surface Science 605: L57-L60.

308. Sul Y-T (2010) Electrochemical growth behavior, surface properties, and enhanced in vivo bone response of $\mathrm{TiO} 2$ nanotubes on microstructured surfaces of blasted, screw-shaped titanium implants. International Journal of Nanomedicine 5: 87100.

309. Wouters O, Vellinga WP, van Tijum R, De Hosson JTM (2006) Effects of crystal structure and grain orientation on the roughness of deformed polycrystalline metals. Acta Materialia 54: 2813-2821.

310. B H Energy Dispersive Spectroscopy on the SEM.

311. A S Energy Dispersive X-Ray Spectrometry -- EDS Instrumentation \& Signal Detection.

312. Linsebigler AL, Lu GQ, Yates JT (1995) PHOTOCATALYSIS ON TIO2 SURFACES - PRINCIPLES, MECHANISMS, AND SELECTED RESULTS. Chemical Reviews 95: 735-758.

313. Ketteler G, Yamamoto S, Bluhm H, Andersson K, Starr DE, et al. (2007) The nature of water nucleation sites on $\mathrm{TiO} 2(110)$ surfaces revealed by ambient pressure $\mathrm{X}$ ray photoelectron spectroscopy. Journal of Physical Chemistry C 111: 8278-8282.

314. Shin HJ, Jeong DK, Lee JG, Sung MM, Kim JY (2004) Formation of TiO2 and $\mathrm{ZrO} 2$ nanotubes using atomic layer deposition with ultraprecise control of the wall thickness. Advanced Materials 16: 1197-+. 
315. Krasowska M, Zawala J, Malysa K (2009) Air at hydrophobic surfaces and kinetics of three phase contact formation. Advances in Colloid and Interface Science 14748: 155-169.

316. Asmatulu R (2008) Improving the dewetability characteristics of hydrophobic fine particles by air bubble entrapments. Powder Technology 186: 184-188.

317. Park NG, van de Lagemaat J, Frank AJ (2000) Comparison of dye-sensitized rutileand anatase-based TiO2 solar cells. Journal of Physical Chemistry B 104: 89898994.

318. Wagemaker M, van de Krol R, Kentgens APM, van Well AA, Mulder FM (2001) Two phase morphology limits lithium diffusion in TiO2 (anatase): A Li-7 MAS NMR study. Journal of the American Chemical Society 123: 11454-11461.

319. Hu YS, Kienle L, Guo YG, Maier J (2006) High lithium electroactivity of nanometer-sized rutile TiO2. Advanced Materials 18: 1421-+.

320. Yang HG, Sun CH, Qiao SZ, Zou J, Liu G, et al. (2008) Anatase TiO(2) single crystals with a large percentage of reactive facets. Nature 453: 638-U634.

321. Mishra S, Gupta SK, Jha PK, Pratap A (2010) Study of dimension dependent diffusion coefficient of titanium dioxide nanoparticles. Materials Chemistry and Physics 123: 791-794.

322. Dozzi M SE (2013) Specific Facets-Dominated Anatase TiO2: Fluorine-Mediated Synthesis and Photoactivity. Catalysts 3: 455-485.

323. Wu Y, Xing M, Tian B, Zhang J, Chen F (2010) Preparation of nitrogen and fluorine co-doped mesoporous $\mathrm{TiO} 2$ microsphere and photodegradation of acid orange 7 under visible light. Chemical Engineering Journal 162: 710-717.

324. Chen JS, Tan YL, Li CM, Cheah YL, Luan D, et al. (2010) Constructing Hierarchical Spheres from Large Ultrathin Anatase TiO2 Nanosheets with Nearly 100\% Exposed (001) Facets for Fast Reversible Lithium Storage. Journal of the American Chemical Society 132: 6124-6130.

325. S S (2011) Anodization Growth and Integration of Titanium Dioxide. Master of Science Thesis. 
326. Wei W, Berger S, Hauser C, Meyer K, Yang M, et al. (2010) Transition of TiO2 nanotubes to nanopores for electrolytes with very low water contents. Electrochemistry Communications 12: 1184-1186.

327. Macak JM, Hildebrand H, Marten-Jahns U, Schmuki P (2008) Mechanistic aspects and growth of large diameter self-organized $\mathrm{TiO} 2$ nanotubes. Journal of Electroanalytical Chemistry 621: 254-266.

328. Manivasagam G, Dhinasekaran D, Rajamanickan A (2010) Biomedical implants: Corrosion and its prevention - A review. Recent Patents on Corrosion Science 2: 40-54.

329. Park J, Lakes R (1992) Hard tissue replacement II: joints and teeth. Biomaterials: An introduction. New York, New York: Plenum. pp. 317-354.

330. Hansen D (2008) Metal corrosion in the human body: The ultimate bio-corrosion scenario. The Electrochemical Society: Interface: 31-34.

331. Manivasagam G, Singh AK, Asokamani R, Gogia AK (2009) Ti based biomaterials, the ultimate choice for orthopaedic implants - A review. Progress in Materials Science 54: 397-425.

332. Das K, Bose S, Bandyopadhyay A (2009) TiO2 nanotubes on Ti: Influence of nanoscale morphology on bone cell-materials interaction. Journal of Biomedical Materials Research 90: 225-237.

333. Roy P, Berger S, Schmuki P (2011) TiO2 nanotubes: Synthesis and applications. Angewandte Chemie 50: 2904-2939.

334. Minagar S, Wang J, Berndt C, Ivanova E, Wen C (2013) Cell response of anodized nanotubes on titanium and titanium alloys - A review. Journal of Biomedical Materials Research 101A: 2726-2739.

335. Gutwein L (2004) Increased viable osteoblast density in the presence of nanophase compared to conventional alumina and titania particles. Biomaterials 25: 41754183.

336. Webster T, Ejiofor J (2004) Increased osteoblast adhesion on nanophase metals: Ti, Ti6Al4V, and CoCrMo. Biomaterials 25: 4731-4739. 
337. Oh S, Daraio C, Chen L-H, Pisanic T, Finones R, et al. (2006) Significantly accelerated osteoblast cell growth on aligned $\mathrm{TiO} 2$ nanotubes. Journal of Biomedical Materials Research 78A: 97-103.

338. Shin D, Shokuhfar T, Choi C, Lee S, Friedrich C (2011) Wettability changes of $\mathrm{TiO} 2$ nanotube surfaces. Nanotechnology 22: 1-7.

339. Wang N, Li H, Lu W, Li J, Wang J, et al. (2011) Effects of TiO2 nanotubes with different diameters on gene expression and osseointegration of implants in minipigs. Biomaterials 32: 6900-6911.

340. von Wilmosky C, Bauer S, Lutz R, Meisel M, Neukam F, et al. (2009) In vivo evaluation of anodic $\mathrm{TiO} 2$ nanotubes: An experimental study in the pig. Journal of Biomedical Materials Research B 89: 165-167.

341. Saldana L, Vilaboa N, Valles G, Gonzalez-Cabrero J, Munuera L (2005) Osteoblast response to thermally oxidized Ti6A14V alloy. Journal of Biomedical Materials Research 73A: 97-107.

342. Yu W-q, Zhang Y, Jiang X-q, Zhang F-q (2010) In vitro behavior of MC3T3-E1 preosteoblast with different annealing temperature Titania nanotubes. Oral Diseases 16: 624-630.

343. Kahn MA, Williams RL, Wiliams DF (1999) The corrosion behavior of Ti-6Al-4V, Ti-6Al-7Nb and Ti-13Nb-13Zr in protein solutions. Biomaterials 20: 631-637.

344. Alves V, Reis R, Santos I, Souza D, Goncalves T, et al. (2009) In situ impedance spectroscopy study of the electrochemical corrosion of $\mathrm{Ti}$ and $\mathrm{Ti}-6 \mathrm{Al}-4 \mathrm{~V}$ in simulated body fluid at $25 \mathrm{C}$ and 37 C. Corrosion Science 51: 2473-2482.

345. Guleryuz H, Cimenoglu H (2004) Effect of thermal oxidation on corrosion and corrosion-wear behaviour of a Ti-6Al-4V alloy. Biomaterials 25: 3325-3333.

346. Kumar S, Narayanan T, Raman S, Seshadri S (2009) Thermal oxidation of cp-Ti: Evaluation of characteristics and corrosion resistance as a function of treatment time. Materials Science and Engineering C 29: 1942-1949.

347. Yu W-q, Qiu J, Ling X, Zhang F-q (2009) Corrosion behaviors of TiO2 nanotube layers on titanium in Hank's solution. Biomedical Materials 4: 1-6. 
348. Bai Y, Park I, Park H, Lee M, Bae T, et al. (2010) The effect of annealing temperatures on surface properties, hydroxyapatite growth and cell behaviors of TiO2 nanotubes. Surface and Interface Analysis 43: 998-1005.

349. Yu W-q, Qiu J, Zhang F-q (2011) In vitro corrosion study of different TiO2 nanotube layers on titanium in solution with serum proteins. Colloids and Surfaces B: Biointerfaces 84: 400-405.

350. Park H, Park I, Kim K, Jeon W, Park B, et al. (2010) Bioactive and electrochemical characterization of $\mathrm{TiO} 2$ nanotubes on titanium via anodic oxidation. Electrochemica Acta 55: 6109-6114.

351. Yang B, Ng C, Fung M, Ling C, Djurisic A, et al. (2011) Annealing study of titanium oxide nanotube arrays. Materials Chemistry and Physics 130: 1227-1231.

352. Velten D, Biehl V, Aubertin F, Valeske B, Possart W, et al. (2001) Preparation of $\mathrm{TiO} 2$ layer on $\mathrm{Cp}-\mathrm{TI}$ and Ti-V by thermal and anodiz oxidation and by sol-gel. Journal of Biomedical Materials Research 59: 18-28.

353. Derbali L (2012) Vanadium-based antireflection coated on multicrystalline silicon acting as a passivating layer. Solar Energy 86: 1504-1510.

354. Adamczyk A (2012) The FTIR studies of gels and thin films of Al2O3-TiO2 and Al2O3-TiO2-SiO2 systems. Spectrochim Acta Mol Biomol Spectrosc 89: 11-17.

355. Bezrodna T (2004) IR-analysis of H-bonded H2O on the pure TiO2 surface. Journal of Molecular Structure 700: 175-181.

356. Riesgraf D, May M (1978) IR spectra of aluminum hydroxide chloride. Applied Spectroscopy 32: 362-366.

357. Walivaara B, Aronsson B, Rodahl M, Lausmaa J, Tengvall P (1999) Titanium with different oxides: in vitro studies of protein adsorption and contact activation. Biomaterials 15: 827-834.

358. Baboian R (2005) Corrosion tests and standards: Application and interpretation. ASTM International.

359. Regonini D, Bowen CR, Jaroenworaluck A, Stevens R (2013) A review of growth mechanism, structure, and crystallinity of anodized $\mathrm{TiO} 2$ nanotubes. Materials Science and Engineering R 74: 377-406. 
360. Saji V, Choe H (2009) Electrochemical corrosion behavior of nanotubular Ti-13Nb13Zr alloy in ringer's solution. Corrosion Science 51: 1658-1663.

361. Haeri M, Wollert T, Langford G, Gilbert J (2012) Electrochemical control of cell death by reduction-induced intrinsic apoptosis and oxidation-induced necrosis on CoCrMo alloy in vitro. Biomaterials 33.

362. Chen H, Dai M, Bai X, Li W (1989) Study on the anodic oxidation on Ti and Ta6V (Ti6Al4V) by ion-implanted Xe ions markers and O18 tracing techniques. MRS Proceedings 157.

363. Reis F, Stafiej J (2007) Scaling behavior in corrosion and growth of a passive film. Physical Review E 76: 1-7.

364. Uberuaga B, Bai X-M (2011) Defects in rutile and anatase polymorphs of TiO2: kinetics and thermodynamics near grain boundaries. Journal of Physics: Condensed Matter 23: 1-11. 